\title{
Innovative production of nuclear fuel by microwave internal gelation
}

MARÍA CABANES SEMPERE 



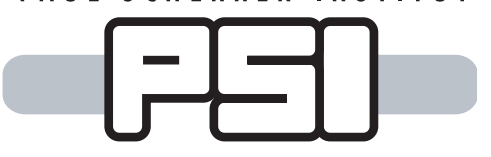

\title{
Innovative Production of Nuclear Fuel by Microwave Internal Gelation
}

\section{DOCTORAL THESIS}

\author{
by \\ Maria Cabanes Sempere
}

Supervisors:

Prof. José Manuel Catalá Civera

Dr. Felipe Laureano Peñaranda Foix

Dr. Manuel Alexandre Pouchon

València, Spain

June 2013 
UNIVERSITAT

POLITÉCNICA

DE VALÈNCIA

First Edition, 2013

(c) Maria Cabanes Sempere

(c) of the present edition:

Editorial Universitat Politècnica de València

www.lalibreria.upv.es

ISBN: 978-84-9048-133-2 (printed version)

Publishing reference: 5657

Any unauthorized copying, distribution, marketing, editing, and in general any other exploitation, for whatever reason, of this piece of work or any part thereof, is strictly prohibited without the authors' expressed and written permission. 
Als meus tres pilars

Marisa, Aranza $i$ Vicent 

"El que ens agrada no és el nostre camí, com tampoc ho és allò que no ens agrada.

De vegades, el nostre rumb

pot estar en allò que ens provoca indiferència,

en allò que no ens apassiona

o en allò que avorrim."

"What we like is not our destiny,

nor is what we dislike.

Sometimes our path

will come across things which cause us indifference,

things we are uncomfortable with

or things that bore us."

Si tu em dius vine ho deixo tot... Però digue'm vine

Albert Espinosa 



\section{Abstract}

In the continuous aim to reduce the amount of minor actinides (MA) from the spent fuel of Light Water Reactors (LWR) and therefore reduce its radiotoxicity (radioactive toxicity), new nuclear fuel concepts have been developed. Sphere-Pac (SP) fuel gives the opportunity to reintroduce the MA in a fuel matrix and to burn them in a fast reactor, which facilitates a multi-cycle because of its breeding feature, or in a subcritical fast system, i.e. an Accelerator Driven System (ADS) where its sub-criticality allows higher MA contents than a normal fast reactor reducing efficiently the radiotoxicity in one step.

SP fuel is produced from the base solution (already containing all the elements) by internal gelation, which guarantees a good material homogeneity and a lower contamination risk compared to the classical pellet fabrication, avoiding presses and grinding machines. The internal gelation is an aqueous chemical reaction occurring when the solution is heated up to $80 \pm 5^{\circ} \mathrm{C}$. When performing the internal gelation process with electromagnetic heating, some advantages appear with respect to the traditionally heating through conduction by contact of the sample with hot silicon oil: the recycling step of the oil and the organic solvents necessary to clean the particles from oil are avoided. In the Microwave Internal Gelation (MIG) unit, the microwaves represent a much simpler and safer alternative: the contactless volumetric heating facilitates the remote production of the fuel in hot cells and furthermore reduces the contaminated liquid waste.

The fuel related project called Platform for Innovative Nuclear FuEls (PINE), in which this thesis is embedded, aims for the production of SPfuel by MIG. In the MIG system, the heating time is very short (in the order of tens of milliseconds), therefore the microwave heating parameters have to be optimized and a good knowledge of the interaction between the microwaves and the samples must be achieved.

In the first part of this dissertation a finite difference time domain (FDTD) thermal model capable to determine over each instant about the thermal behaviour of a definite point inside a material during heat processing is investigated. A detailed overview of the most relevant parameters on the model including the boundary conditions (e.g. convection) is presented. Furthermore, the model is analytically implemented and validated 
with different techniques: a theoretical based physically validation, a partial differential equations (PDEtools) based validation and a validation with examples from the literature.

Secondly, possible microwave cavity designs for MIG are researched. The cavities (selection of modes, resonant frequency, $Q$-factor, etc.) and its subsequent characterization for the coupling of energy are explained. Furthermore, the power transfer mechanisms of the cavities are explained using the perturbation method to analyse the losses when a dielectric sample is placed inside a cavity. The developed power transfer model delivers the microwave heat generation rate which is applied to the FDTD thermal model mentioned in the previous paragraph. The analytical results provide a positive impression about the feasibility of producing gelated spheres by MIG.

Next, the main parameters dealing with the heating of a material by microwaves are introduced. A new procedure that enables the measurement of dielectric properties of aqueous droplets freely falling through a microwave cavity is developed. The experimental setup is presented and several experiments prove its feasibility. The measured dielectric properties are afterwards included in the perturbation and thermal models with the main intention of determining the absorbed power by the material in form of drops and the reached temperature.

In the last part the MIG system for the laboratory practice of the high frequency heating applied to the PINE project is implemented. Each device is characterized for a power study precedent to the MIG system assembly, avoiding then failures when putting the system into operation. In addition, the experimental techniques and the results are reported. Successful production of gelated spheres shows the favourable usage of microwave for the production of SP-fuel by internal gelation.

Keywords: Microwave Internal Gelation, Sphere-Pac Fuel, Resonant Cavities, Resonant Frequency, Q-factor, Dielectric Properties, Perturbation Method. 


\section{Resumen}

El continuo afán por reducir la cantidad de actínidos minoritarios (MA) procedentes del combustible quemado en los reactores de agua ligera (Light Water Reactor, LWR) y de esa forma reducir la radiotoxicidad, ha llevado a desarrollar nuevos conceptos de combustible nuclear. El nuevo combustible por empaquetamiento de esferas (Sphere-Pac, SP) ofrece la oportunidad de reintroducir los $\mathrm{MA}$ en una matriz y quemarlos en un reactor rápido de neutrones, donde se facilitan ciclos múltiples por transmutación de elementos. Este combustible se puede utilizar también en un sistema subcrítico rápido de neutrones, es decir, un sistema nuclear accionado por un acelerador de partículas (Accelerator Driven System, ADS), donde la subcriticidad (seguridad de parada del reactor) permite utilizar combustibles con mayor contenido de MA que en un reactor normal, reduciendo eficazmente en un solo paso la radiotoxicidad.

El combustible SP se produce a partir de una solución base (formada por metales y elementos químicos) mediante un proceso de gelificación interna. Este proceso garantiza una buena homogeneidad del producto final y un riesgo de contaminación mucho menor si se compara con la fabricación clásica de pellets (combustible comprimido), puesto que se evita el uso de prensas y amoladoras. La gelificación interna es una reacción química acuosa que se produce al calentar la solución hasta $80 \pm 5^{\circ} \mathrm{C}$. Cuando se realiza el proceso por calentamiento electromagnético, se observan algunas ventajas con respecto al calentamiento tradicional por conducción (contacto de la muestra con aceite de silicio precalentado): se evita la etapa de reciclado del aceite y de los disolventes orgánicos necesarios para eliminar el aceite de la superficie de las partículas producidas. En la unidad de gelificación interna por microondas (Microwave Internal Gelation, MIG), las microondas representan una alternativa mucho más simple y segura: el calentamiento volumétrico sin contacto facilita la producción a distancia del combustible en celdas calientes y además reduce los residuos de líquido contaminado.

Esta tesis se enmarca dentro del proyecto Platform for Innovative $\mathrm{Nu}$ clear FuEls (PINE), que tiene como objetivo fundamental la producción de combustible SP por MIG. En el sistema MIG, el tiempo de calentamiento es muy corto (del orden de decenas de milisegundos), por lo que se deben optimizar los parámetros que contribuyen al calentamiento por microondas 
y es necesario conocer en profundidad la interacción entre las microondas y las muestras.

En la primera parte de este trabajo se investiga un modelo térmico basado en diferencias finitas en el dominio del tiempo (FDTD), el cual es capaz de determinar, en cada instante durante el proceso de calentamiento, el comportamiento térmico de un punto definido dentro del material que se calienta. Además se presenta una descripción detallada de los parámetros más relevantes del modelo, incluyendo las condiciones de contorno (entre ellas la convección). Por otra parte, se implementa analíticamente y se valida con diferentes técnicas: una basada en teoría de la física, otra basada en la herramienta de ecuaciones diferenciales parciales (PDEtools) y la última basada en ejemplos encontrados en la literatura.

En segundo lugar, se investigan los posibles diseños de cavidades de microondas para su aplicación en MIG. Tanto las cavidades (selección de los modos, frecuencia de resonancia, factores de calidad, etc.) como su posterior caracterización, se detallan con el objetivo de especificar el acoplamiento de energía. Los mecanismos de transferencia de energía de las cavidades se explican utilizando el método de perturbación, con el que además se analizan las pérdidas de la cavidad cuando se coloca una muestra dieléctrica en su interior. Con el modelo de transferencia de energía desarrollado, se obtiene la tasa de generación de calor por microondas, que se aplica al modelo térmico FDTD mencionado anteriormente. Los resultados analíticos demuestran la viabilidad de producir esferas gelificadas por MIG.

Seguidamente se introducen los principales parámetros relacionados con el calentamiento de un material por microondas, es decir, las propiedades dieléctricas. Se desarrolla un nuevo procedimiento que permite medir estas propiedades en gotas que caen libremente a través de una cavidad de microondas. Se presenta el montaje experimental, cuya viabilidad se prueba a través de diferentes experimentos. Las propiedades dieléctricas medidas se incluyen en los modelos (perturbacional y térmico) con la intención de determinar la potencia absorbida por la sustancia (en forma de gotas) y la temperatura que alcanza.

En la última parte se presenta la implementación del sistema MIG aplicada al proyecto PINE, fundamental para la práctica de calentamiento (basado en frecuencias altas) dentro del laboratorio. Las propiedades de cada dispositivo se evaluan para realizar un estudio de potencia antes del ensamblaje del sistema MIG. De esa forma se evitan fallos al poner el sistema en funcionamiento. Además se aportan las técnicas experimentales y 
los resultados. La producción con éxito de esferas gelificadas demuestra, sin duda, el uso favorable de las microondas en la producción de combustible SP por gelificación interna.

Palabras Clave: Gelificación Interna por Microondas, Combustible por Empaquetamiento de Esferas, Cavidades Resonantes, Frecuencia de Resonancia, Factor de Calidad, Propiedades Dieléctricas, Método Perturbacional. 

L'esforç continuat per reduir la quantitat d'actínids minoritaris (MA) provinents del combustible cremat als reactors d'aigua lleugera (Light Water Reactor, LWR) i d'aquesta manera reduir la radiotoxicitat, ha dut al desenvolupament de nous conceptes de combustible nuclear. El combustible per empaquetament d'esferes (Sphere-Pac, SP) ofereix l'oportunitat de reintroduir els MA en una matriu pròpia i cremar-los en un reactor ràpid de neutrons, el qual facilita cicles múltiples per transmutació d'elements. Aquest combustible també pot ser utilitzat en un sistema subcrític ràpid de neutrons, és a dir, un sistema nuclear accionat per un accelerador de partícules (Accelerator Driven System, ADS), on la subcriticitat (seguretat de parada del reactor) permet utilitzar combustibles amb major contingut de MA que a un reactor normal amb la consegüent reducció de la radiotoxicitat en un sol pas.

El combustible SP es produeix a partir d'una solució base, formada per metalls i elements químics, per gelificació interna. Aquest mètode garanteix l'homogeneïtat del producte final i un risc de contaminació molt menor si es compara amb la fabricació clàssica de pellets (combustible comprimit) ja que s'evita l'ús de premses i esmoladores. La gelificació interna és una reacció química aquosa que es produeix en escalfar la solució fins $80 \pm 5^{\circ} \mathrm{C}$. Quan es realitza el procés per escalfament electromagnètic, apareixen alguns avantatges respecte al sistema tradicional per conducció, contacte de la mostra amb oli de silici precalfat: s'evita l'etapa de reciclatge de l'oli i dels dissolvents orgànics necessaris per eliminar l'oli de la superfície de les partícules produïdes. A la unitat de gelificació interna per microones (Microwave Internal Gelation, MIG), les microones representen una alternativa simple i segura: l'escalfament volumètric sense contacte facilita la producció a distància del combustible en cel-les calentes i a més redueix els residus de líquid contaminat.

Aquesta tesi està emmarcada dins del projecte Platform for Innovative Nuclear Fuels (PINE), l'objectiu fonamental del qual és la producció de combustible SP per MIG. Al sistema MIG, el temps d'escalfament és molt curt (desenes de mil-lisegons), per tant, s'han d'optimitzar els paràmetres que contribueixen al calfament per microones i s'ha de conèixer en profunditat la interacció entre les microones i les mostres. 
A la primera part d'aquest treball s'investiga un model tèrmic basat en diferències finites en el domini del temps (FDTD), el qual és capaç de determinar, a cada instant durant tot el procés d'escalfament, el comportament tèrmic d'un punt definit dins del material calfat. A més, es presenta una descripció detallada dels paràmetres més rellevants del model, on s'inclouen les condicions de contorn, entre d'altres la convecció. Per una altra banda, el model s'implementa analíticament i es valida amb diferents tècniques: una basada en teoria de la física, la segona basada en l'eina d'equacions diferencials parcials (PDEtools) i per últim una basada en exemples de la literatura.

En segon lloc, s'investiguen els possibles dissenys de cavitats de microones per a la seua aplicació en MIG. Tant les cavitats (selecció dels modes, freqüència de ressonància, factors de qualitat, etc.) com la seua posterior caracterització, es detallen amb l'objectiu d'especificar l'acoblament d'energia. Els mecanismes de transferència d'energia de les cavitats s'expliquen amb l'ús del mètode de pertorbació, amb què s'analitzen les pèrdues de la cavitat quan es col-loca una mostra dielèctrica en el seu interior. Amb el model de transferència d'energia desenvolupat s'obté la taxa de generació de calor per microones que s'aplica al model tèrmic FDTD esmentat al paràgraf anterior. Els resultats analítics proporcionen dades molt positives pel que fa a la viabilitat en el procés de producció d'esferes gelificades per MIG.

Seguidament s'introdueixen els principals paràmetres relacionats amb l'escalfament d'un material per microones, és a dir, les propietats dielèctriques. Es desenvolupa un nou procediment que permet la medició d'aquestes propietats en gotes que cauen lliurement a través d'una cavitat de microones. Es presenta el muntatge experimental i la viabilitat es prova mitjançant diferents experiments. Les propietats dielèctriques mesurades s'inclouen als models (pertorbacional i tèrmic) amb la intenció de determinar la potència absorbida pel material en forma de gotes i, a més, la temperatura que aconsegueix.

Dins l'última part del treball es presenta la implementació del sistema MIG aplicada al projecte PINE, fonamental per a la pràctica d'escalfament (en freqüències altes) dins del laboratori. Les propietats de cada dispositiu s'avaluen per realitzar un estudi de potència abans de l'acoblament del sistema MIG. D'aquesta manera s'eviten errors quan es posa el sistema en funcionament. A més, es presenten les tècniques experimentals i els resultats. La producció amb èxit d'esferes gelificades demostra, sens dubte, l'ús 
favorable de les microones en la producció de combustible SP per gelificació interna.

Paraules Clau: Gelificació Interna per Microones, Combustible per Empaquetament d'Esferes, Cavitats Ressonants, Freqüència de Resonància, Factor de Qualitat, Propietats Dielèctriques, Mètode Pertorbacional. 



\section{Zusammenfassung}

Beim fortlaufenden Ziel die Menge des Transuranabfalls (Minore Actinoide, MA) von den abgebrannten Brennelementen der Leichtwasserreaktoren (LWR) zu verringern und somit ihre Radiotoxizität (radioaktive Toxizität) zu reduzieren, sind neue Kernbrennstoffkonzepte entwickelt worden. Der so genannte Sphere-pac (SP, zu Deutsch: Kugelpackungs-) Brennstoff gibt die Möglichkeit die MA in einer Brennstoff-Matrix wieder einzuführen und sie in einem schnellen Reaktor Zyklus zu verbrennen. Dieser Brennstoff kann auch in einem unterkritischen schnellen System verbrannt werden, d.h. einem Accelerator Driven System (ADS), wo die sub-Kritikalität höhere MA Inhalte als in einem normalen schnellen Reaktor ermöglicht und effizient die Radiotoxizität in einem Schritt reduziert.

SP Brennstoff wird aus der Ausgangslösung (die bereits alle Elemente enthält) durch interne Gelierung hergestellt. Diese Methode gewährt eine gute Homogenität des Materials und weniger Kontaminationsrisiko im Vergleich zu der klassischen Pellet Herstellung, da Pressen und Schleifmaschinen vermieden werden. Die interne Gelierung ist eine wässrige chemische Reaktion, die durch eine Temperaturerhöhung von $80 \pm 5^{\circ} \mathrm{C}$ ausgelöst wird. Die Durchführung des Prozesses mit Mikrowellenheizung hat einige Vorteile gegenüber der traditionellen Erwärmung (Kontakt der Probe mit heißem Silikonöl): der Rückführungsschritt des Öls und der organischen Lösungsmittel, um das Öl beziehungsweise die Partikel vom Öl zu reinigen, wird vermieden. In der Anlafe zur Mikrowellen Internen Gelierung (MIG) stellen die Mikrowellen eine einfache und sichere Alternative dar: die kontaktlose volumetrische Erwärmung erleichtert die ferngesteuerte Produktion des Brennstoffs in heißen Zellen und verringert außerdem kontaminierte flüssige Abfälle.

Das Projekt Platform for Innovative Nuclear Fuels (PINE, zu Deutsch: fortgeschrittene Brennstoffe für zukünftige Reaktoren), in welchem diese Doktorarbeit eingebettet ist, has die Produktion von SP-Brennstoff durch MIG zum Ziel. Im MIG-System ist die Aufheizzeit sehr kurz (in der Größenordnung von einigen zehn Millisekunden). Infolgedessen sollen die Mikrowellenerwärmungsparameter optimiert werden und eine gute Kenntnis der Interaktion zwischen den Mikrowellen und der Proben erlangt werden. 
Im ersten Teil dieser Dissertation wird eine Finite-Differenzen-Methode im Zeitbereich (Finite Difference Time Domain, FDTD) für das thermisches Modell erforscht. Dieses Modell untersucht während der Wärmebehandlung zu jedem Zeitpunkt das thermische Verhalten einer bestimmten Stelle innerhalb eines Materials. Eine detaillierte Übersicht der wichtigsten Parameter des Modells einschließlich der Randbedingungen (z.B. Konvektion) wird vorgestellt. Weiterhin wird das Modell analytisch implementiert und mit verschiedenen Techniken übergeprüft: eine Theoretisch auf physikalische Gesetze basierende Überprüfung, eine Überprüfung gestützt auf ein Programm basierend auf partielle Differentialgleichungen (PDEtools) und eine Überprüfung mit Beispiele aus der Literatur.

Im zweiten Teil werden mögliche Mikrowellenhohlraum Designs für MIG erforscht. Die Hohlräume (Auswahl von Modus, Resonanzfrequenz, QFaktor, usw.) und die anschließende Charakterisierung für die Einkopplung der Energie werden erläutert. Desweiteren werden die Leistungsübertragungsmechanismen der Hohlräume anhand der Perturbationsmethode ermittelt um die Verluste zu analysieren, wenn eine dielektrische Probe in einem Hohlraum platziert wird. Das entwickelte Leistungsübertragungsmodell liefert die Mikrowellenwärmeerzeugungsrate, die im vorhergehenden Absatz erwähntem FDTD-thermischen Modell benutzt wird. Die analytischen Ergebnisse resultieren in einer Machbarkeit der Kügelchenherstellung mittels MIG.

Anschließend werden die zur Erwärmung eines Materials durch Mikrowellen relevanten Hauptparameter eingeführt. Ein neues Verfahren, welches die Messung der dielektrischen Eigenschaften von frei fallenden wässrigen Tröpfchen durch einen Mikrowellenhohlraum ermöglicht, wurde im Rahmen dieser Dissertation entwickelt. Der experimentelle Aufbau wird hier vorgestellt wobei mehrere Experimente die Machbarkeit beweisen. Die gemessenen dielektrischen Eigenschaften werden danach in den Perturbationsund Thermischen-Modellen eingefügt, mit dem Hauptziel, die Leistung zu bestimmen, welche durch das Material aufgenommen wird (in Form von Tropfen), und zu berrechnen welche Temperatur erreicht wird.

Der letzten Teil zeigt die MIG-System Implementation wie sie im PINEProjekt für die Laborpraxis der Hochfrequenzheizvorrichtung realisiert wurde. Vor der Montage des gesamten MIG-System durchluft jedes Gerät eine Leistungsstudie um Fehler zu vermeiden, wenn das System in Betrieb ist. Schließlich wird über die experimentellen Techniken und Ergebnisse berichtet. Die erfolgreiche Produktion von gelierten Kugelnchen zeigt, dass die 
Herstellung von SP-Brennstoff durch interne Gelierung mit Mikrowellenheizung machbar ist.

Stichworte: Mikrowellen Interne Gelierung, Sphere-pac Brennstoff, Resonanzhohlräume, Resonanzfrequenz, Q-Faktor, dielektrische Eigenschaften, Perturbationsmethode. 



\section{Acknowledgements}

I would really like to thank all those who made this thesis possible. First and foremost I offer my sincerest gratitude to my supervisors, Dr. Manuel A. Pouchon at the Paul Scherrer Institut (PSI, Switzerland) and Prof. José M. Catalá Civera and Dr. Felipe L. Peñaranda Foix at the Universitat Politècnica de València (UPV, Spain), who supported me throughout this thesis with their knowledge and advice whilst allowing me the room to work in my own way.

It is a pleasure for me to thank Dr. Michael D. Janezic from the National Institute of Standards and Technology from Boulder (Colorado, US), Prof. Cristina Leonelli from the University of Modena \& Reggio Emilia (Italy) and Prof. Juan Monzó Cabrera from the Universidad Politécnica de Cartagena (Spain) for serving as reviewers of this thesis and for providing me with very useful comments that helped to improve the final manuscript. I thank Dr. Johannes Bertsch, who apart from being a member of the committee, kindly supported me during my work at PSI in Switzerland.

I am very grateful to those people who were involved in the PINE project, especially to Dr. Sebastién Vaucher and Dr. Kotaro Ishizaki from EMPA Thun (Switzerland) and foremost to Dr. Cedric Cozzo from PSI, who gave me significant advices and encouragement to carry out this thesis in and outside PSI. A special mention goes to the people from the Instituto de Aplicaciones de las Tecnologías de la Información y de las Comunicaciones Avanzadas (ITACA): Dr. Beatriz García, Pedro Plaza and José Daniel Gutiérrez for their technical advices in the laboratory during my stay in the UPV and Toni Canós for sharing with me his wide experience about the perturbation method and for his collaboration in one of the papers. It was a pleasure to work with all of them. I thank also the people from ESA, who contributed with the X-Band components, which I required for the experiments in the UPV.

I would like to show my gratitude to all the people at the Paul Scherrer Institut that shared my daily work at the Laboratory of Nuclear Mate- 
rials. In particular, I would like to thank Dr. Péter Dusán Ispánovity, who helped me to understand new concepts during the first period of this thesis, Barbara Furger for helping me in all the administrative tasks, my nice officemate Dr. Ning Gao and Carl Beard for his grate introduction in the CST Microwave Studio software. A special mention to Sandra Ruchti and Sybille Traber to let me improve my communication skills during the work in the PSI Forum.

I owe my deepest gratitude to Dr. Basilio Pueo from the Universitat d'Alacant. He introduced me in the scientific world being the supervisor of my bachelor thesis and encouraged me during my whole scientific career providing relevant advices and technical support. Thanks to him for also helping me in the design of the thesis cover.

I am very thankful to all the people at the Universitat Politècnica de Valencia that worked with me during my Master studies. In particular, I would like to thank Prof. Salvador Sales and Prof. Jose Javier López and my colleagues and good friends: Sandra Roger, Max Cobos, Jonás Porcar, Mohamed Maher and David Martínez. Also thanks to my friends at the Universitat d'Alacant: Miguelón, David, Andrea, Moreno and Roda.

I wish also to thank for the received financial support to the Swiss Competence Center Energy and Mobility (CCEM) under the PINE project and the European Commission through contract no. 295664 regarding the FP7 PELGRIMM Project, as well as contract no. 295825 regarding the FP7-ASGARD Project.

Thanks of course to the people who made me feel in Switzerland like being at home: Adriana Marcucci, Aldo Mozzanica, Mathieu Hursin, Daniela Soltermann, Laure Aimoz, Cedric and Caroline Cozzo, Ana Benedicto, Ainara Irastorza, Henar Rojo, Eva Rodríguez, Nano Ardana, Juanlu Gómez, Carmen Jiménez, Laura Vara, Judith Schenzel, Marie Marques, Marie Laborde, Ángela Saa, Jordi Freixa and Helena Barrio.

Special thanks to the members of the Corporació Musical Primitiva d'Alcoi, to send me the scores for the concerts and not forget me in the distance. It was a pleasure to come back to Alcoi and play the concerts with all of you, in particular: Mariola, Tania C., Rocío, Ana G.; thanks Jaume Jordi, Reyes i Àngel Lluís for your continuous patience and support, for sharing with me your experience and for our interesting discussions. I would also like to thank the members of the Stadtmusik Baden and the Jägermusik Wettingen who gave me the opportunity to play the clarinet also in Switzerland. Thanks to Jenny Sibille for her recommendation of 
playing with them.

Thanks to my dear friends Lliris, Lluïsa, Núria, and Inma for being always with me, for making me always feel that the last time we met was yesterday.

It is a pleasure for me to acknowledge the help and encouragement given by my parents, Vicent and Marisa and by my brothers and sister, Vicente, Nicolás, Gonzalo and Aranza. Thanks dad to come with me to the interview, which gave me this opportunity. Thanks mum to support me and enjoy with me the last conference trip. A warm hug to my grandmas Nati and Maria and kisses to my cousins Celia and Joan, my aunts and uncles Celia, Nando, Nani and Pepito and to the rest of my family.

Finally, I would like to express my deepest gratitude and affection to my loving sister Aranza, who shares with me all in this world, our ups and downs, our bad temper and our tenderness. It is important to have a real trusting relationship in all your moments and situations and with her I have more than confidence.

Thanks to help me to stop my world when I needed and to help me afterwards to get it started stronger.

Maria Cabanes Sempere

June 2013 



\section{Contents}

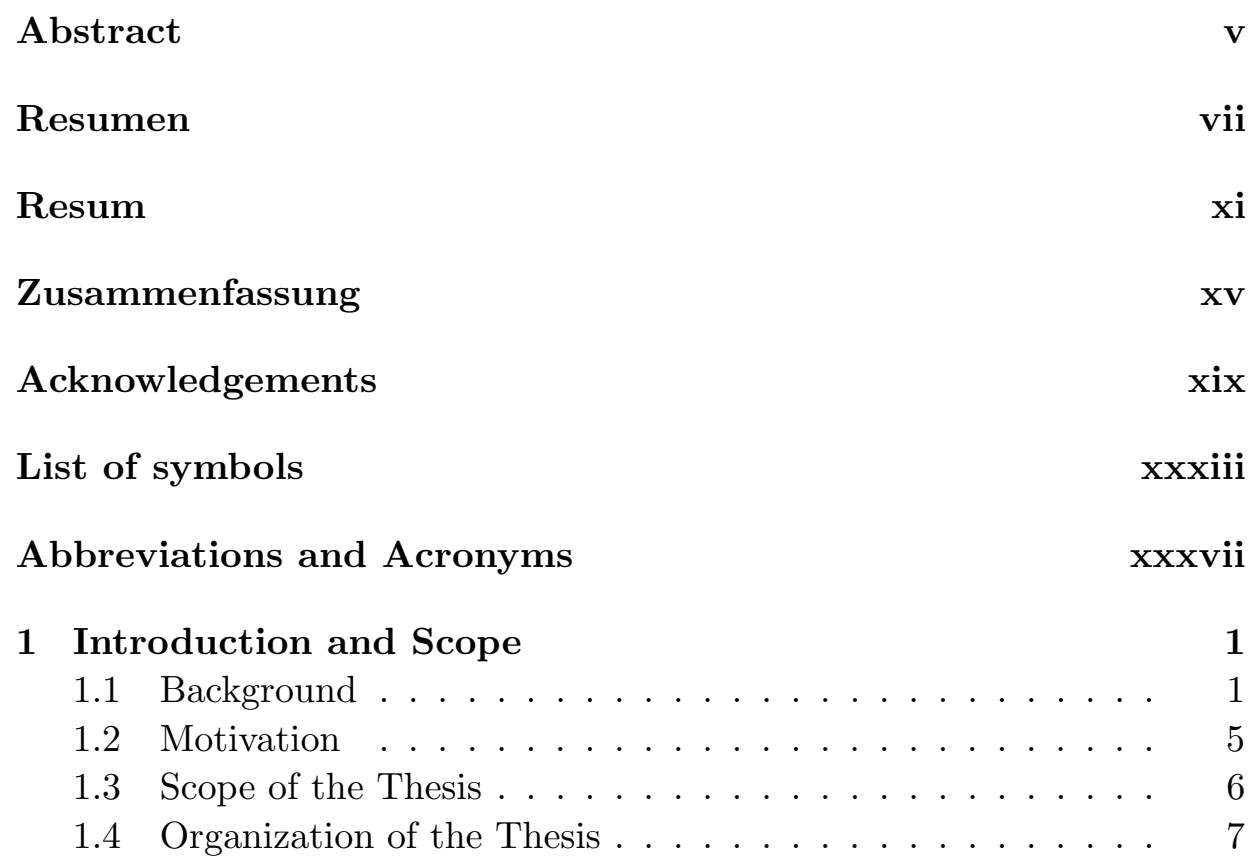

2 Preliminaries and State of the Art 11

2.1 Introduction . . . . . . . . . . . . . . . . 13

2.2 Nuclear Fuel Cycle . . . . . . . . . . . . . . . . . . . . . . 14

2.2.1 Exploration of Uranium Deposits . . . . . . . . . 15

2.2.2 Mining and Milling . . . . . . . . . . . . . . 16

2.2 .3 Refining and Conversion . . . . . . . . . . . . 17

2.2.4 Enrichment . . . . . . . . . . . . . . 17

2.2 .5 Fuel Fabrication . . . . . . . . . . . . . 18

2.2 .6 Electricity Generation . . . . . . . . . . . . 18

2.2 .7 Reprocessing . . . . . . . . . . . . . 20

2.2.8 Geological Disposal and Radiotoxicity . . . . . . . 22

2.3 Solid Nuclear Fuel . . . . . . . . . . . . . . . . . . . . 23

2.3 .1 Pellets . . . . . . . . . . . . . . . 24

$2.3 .2 \quad$ Particle Fuel . . . . . . . . . . . . . . . . 26 
2.4 Sphere-Pac Fuel Production . . . . . . . . . . . . . 27

2.4.1 Water Extraction Process . . . . . . . . . . . 28

2.4 .2 External Gelation . . . . . . . . . . . . . 28

2.4 .3 Internal Gelation . . . . . . . . . . . . . . . . 29

2.5 State of the Art of the Internal Gelation SP Fuel Production 30 2.5.1 Silicon Oil Internal Gelation . . . . . . . . . . . . 30

2.5.2 Microwaves Internal Gelation . . . . . . . . . . . 32

2.6 Conclusions . . . . . . . . . . . . . . . . . 35

3 Thermal Model $\quad 37$

3.1 Introduction . . . . . . . . . . . . . . . . . . 39

3.2 The Heat Equation . . . . . . . . . . . . . . . . . . 42

3.2.1 Definition . . . . . . . . . . . . . . 43

3.2.2 Common Forms of the Heat Equation . . . . . . . . 44

3.3 Heat Equation Solution by Numerical Methods . . . . . . . 46

3.3.1 Finite Difference Time Domain Method . . . . . . . 46

3.4 Boundary Conditions . . . . . . . . . . . . . . . 50

3.4.1 The Dirichlet Boundary Condition . . . . . . . . 50

3.4.2 The von Neumann Boundary Condition . . . . . . 50

3.5 Implementation and Results . . . . . . . . . . . . . . 53

3.5.1 Thermal Parameters Influence . . . . . . . . 55

3.5.2 Influence of the Coordinates System Election . . . . 58

3.6 Validation . . . . . . . . . . . . . . . 60

3.6.1 Physically Validation . . . . . . . . . . . . 60

3.6.2 Matlab Pdetools . . . . . . . . . . . . . . . . 63

3.6.3 Validation from a Literature Example . . . . . . . 65

3.6.4 Validation Results . . . . . . . . . . . . . 66

3.7 Conclusions . . . . . . . . . . . . . . . 67

4 Microwave Cavities $\quad 69$

4.1 Introduction . . . . . . . . . . . . . . . . 71

4.2 Rectangular Cavities . . . . . . . . . . . . . . . 72

4.2.1 Empty Rectangular Cavities . . . . . . . . . . 77

4.2.2 Rectangular Cavities filled with homogeneous dielectrics 79

4.3 Cylindrical Cavities . . . . . . . . . . . . . 83

4.3.1 Empty Cylindrical Cavities . . . . . . . . . . . 84

4.3.2 Cylindrical Cavities completely filled with homogeneous dielectrics . . . . . . . . . . . 86

4.4 Cavity coupling . . . . . . . . . . . . . . . 88 
4.4 .1 The Coupling Factor . . . . . . . . . . . . . . . 89

4.5 Modelling of Microwave Cavities . . . . . . . . . . . . . . . 91

4.5.1 Matlab Modelling . . . . . . . . . . . . . . 91

4.5.2 Electromagnetic Simulation Software . . . . . . . . 101

4.6 Perturbation Analysis of Cavities . . . . . . . . . . . . . . 103

4.6.1 Experimental setup . . . . . . . . . . . . . . 103

4.6.2 Perturbation Method . . . . . . . . . . . . . 103

4.6.3 Power dissipation . . . . . . . . . . . . . . . . . 107

4.6.4 Model Implementation and Results . . . . . . . . . . 110

4.7 Conclusions . . . . . . . . . . . . . . . . . . . . 118

5 Characterization of Materials $\quad 121$

5.1 Introduction . . . . . . . . . . . . . . . . . . . 124

5.2 Dielectric Loss . . . . . . . . . . . . . . . . . . . 124

5.2 .1 Polarization . . . . . . . . . . . . . . . . . 124

5.2 .2 Complex Dielectric Constant . . . . . . . . . . . 126

5.2.3 Volumetric Heating . . . . . . . . . . . . . . . . . 128

5.3 Dielectric Measuring Techniques . . . . . . . . . . . 128

5.4 New Characterization Method of Dielectrics . . . . . . . . 130

5.4.1 Experimental Setup . . . . . . . . . . . . . . 131

5.4 .2 Drop Measurement . . . . . . . . . . . . . . . 134

5.4 .3 Drop Simulation . . . . . . . . . . . . . . 138

5.4.4 Experimental Measurements and Results . . . . . 139

5.5 Perturbation and Thermal Models of Characterized Materials 145

5.6 Conclusions . . . . . . . . . . . . . . . . . . . . 148

6 MIG System 151

6.1 Introduction . . . . . . . . . . . . . . . 153

6.2 MIG System description . . . . . . . . . . . . . . 154

6.3 Equipment . . . . . . . . . . . . . . 156

6.3.1 Design of the microwave cavity . . . . . . . . 156

6.3.2 Microwave generator . . . . . . . . . . . . . . 159

6.3 .3 Amplifier . . . . . . . . . . . . . . . 160

6.3 .4 Isolator . . . . . . . . . . . . . . . . . . . 161

6.3.5 Waveguides . . . . . . . . . . . . . . 162

6.3.6 Adapter . . . . . . . . . . . . . . . . . . 164

6.3.7 Crystal Detector . . . . . . . . . . . . . . . . . 164

6.3 .8 Vector Network Analyser . . . . . . . . . . . . 165

6.3.9 Oscilloscope . . . . . . . . . . . . . . . 166 
6.3.10 Power requirements . . . . . . . . . . . . 167

6.3.11 Calibration . . . . . . . . . . . . 168

6.4 Experimental Techniques . . . . . . . . . . . . . . 169

6.4.1 Rectangular cavity characterization . . . . . . . . 169

6.4 .2 MIG System Assembly . . . . . . . . . . . . . . . . . 171

6.4.3 Heating Experiments . . . . . . . . . . . . . . 175

6.5 Results Analysis . . . . . . . . . . . . . . . . . 176

6.6 Conclusions . . . . . . . . . . . . . . . . . . 180

7 Conclusions 181

7.1 Summary . . . . . . . . . . . . . . . . . . . . . . 184

7.2 Further Work . . . . . . . . . . . . . . . . . . . 185

7.3 List of Publications . . . . . . . . . . . . . . . 186

$\begin{array}{ll}\text { Appendices } & 191\end{array}$

A Convective Heat Transfer Coefficient 193

A.1 Dimensionless groups in heat transfer . . . . . . . . . 195

A.2 Properties of Air . . . . . . . . . . . . 196

A.2.1 Standard Properties . . . . . . . . . . . . 196

A.2.2 Temperature Dependent Properties . . . . . . . . . 197

B Crank Nicholson Development 203

B.1 Rectangular Coordinates . . . . . . . . . . . . . . 207

B.2 Cylindrical Coordinates . . . . . . . . . . . . . 208

B.3 Spherical Coordinates . . . . . . . . . . . . . . . 209

B.4 General equation . . . . . . . . . . . . . . . 211

C Energy and Power Solutions 213

C.1 Calculation of Energies in a Rectangular Empty Cavity . . 215

C.2 Calculation of Power in a Rectangular Empty Cavity . . . . 219

D Bessel Function $\quad 223$

$\begin{array}{lr}\text { Bibliography } & 229\end{array}$ 


\section{List of Figures}

1.1 Comparative flowchart of contact (by silicon oil) and contactless (through microwaves) internal gelation. . . . . . . . . 3

1.2 Block diagram of the Microwave Internal Gelation process with the corresponding manuscript chapters. . . . . . 8

2.1 Nuclear fuel cycle. . . . . . . . . . . . . . . . . 15

2.2 Nuclear fuel electric power generating plant . . . . . . . . 18

2.3 Comparison of 11 representative indicators for various fuel cycle schemes. 1a) Once-through PWR (reference); 1b) 100 $\% \mathrm{PWR}$, spent fuel reprocessed and $\mathrm{Pu}$ reused once; 2a) $100 \%$ PWR, spent fuel reprocessed and multiple reuse of $\mathrm{Pu}$; 3cV1) $100 \%$ fast reactors and fully closed fuel cycle. Source: OECD/NEA, $2006 \ldots \ldots \ldots \ldots$

2.4 Storage model for nuclear spent fuel in the Grimsel Test Site in the Swiss Alps. Picture taken by the author of this manuscript during a NAGRA guided visit in the GTS. . . .

2.5 Ingestion radiotoxicity for spent fuel. a) Uranium ore; b) Uranium and decay products; c) Radiotoxicity of natural uranium and decay products; d) Plutonium and decay products; e) Minor actinides and decay products; f) Fission Products; g) Total. Source: Salvatores, 2011 . . . . . . . . . . 24

2.6 Schema of a fast reactor fuel assembly of pellets. . . . . . . 25

2.7 Comparative schema of a fuel assembly of Vipac (up) and Sphere-pac (down) fuel. . . . . . . . . . . . 26

2.8 Schema of the sol-gel processes: a) internal gelation, b) external gelation, c) water extraction process. . . . . . . .

2.9 Schema of internal gelation production unit used in the past at Paul Scherrer Institut. . . . . . . . . . . . . . . 31

2.10 Design of the microwave applicator by Ledergerber at Paul Scherrer Institut. . . . . . . . . . . . . . . . . 34

3.1 Main thermal factors that influence the heating of the droplet falling in the cavity. . . . . . . . . . . . . 43 
3.2 Representation of the rectangular thermal case: a) 1D drop rectangular profile. b) Temperature profile before heating and c) after heating. The drop is initially cooled, therefore the low water-temperature value in b) . . . . . . . .

3.3 Microwave heat generation rate $\left(Q_{g e n}\right)$ as function of processing time for different drop radius, to achieve a temperature increase of $80 \mathrm{~K} . \ldots \ldots \ldots \ldots$

3.4 Microwave heat generation rate $\left(Q_{g e n}\right)$ as function of processing time for several values of thermal conductivity, to achieve a temperature increase of $80 \mathrm{~K}$ in a $0.5 \mathrm{~mm}$ radius drop. . . . . . . . . . . . . . . .

3.5 Temperature profile of a $0.5 \mathrm{~mm}$ water droplet for several values of convection heat transfer coefficient. . . . . . . 58

3.6 Temperature profile (in rectangular coordinates) of a drop heated by microwaves as function of a) radius, b) radius and processing time. . . . . . . . . . . . .

3.7 Temperature profile (in cylindrical coordinates) of a drop heated by microwaves as function of a) radius, b) radius and processing time. . . . . . . . . . . . .

3.8 Temperature profile (in spherical coordinates) of a drop heated by microwaves as function of a) radius, b) radius and processing time. . . . . . . . . . . . .

3.9 Infinitesimal volume geometry in rectangular coordinates $a$ ), cylindrical coordinates $b$ ) and spherical coordinates $c$ ). . .

3.10 Pdetools meshing for a) rectangular coordinates and b) cylindrical coordinates. . . . . . . . . . . . . .

3.11 Temperature diffusion for the a) rectangular case and b) cylindrical case in Pdetools. . . . . . . . . . . . . . .

3.12 Spatial temperature distribution changing with time within a fuel element. . . . . . . . . . . . . . 66

4.1 Geometry for the rectangular $a$ ) and cylindrical $b$ ) cavities.

4.2 Different schemas of a magnetic coupling on a cylindrical microwave cavity: a) through an iris, b) through a loop. . .

4.3 Different schemas of an electric coupling on a rectangular microwave cavity: a) through an iris, b) through an electric

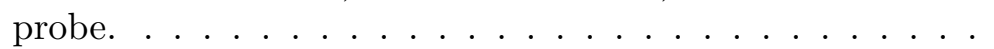


4.4 Smith chart representation of the reflection coefficient for an undercoupled $\left(\beta^{\prime} \ll 1\right)$, critically coupled $\left(\beta^{\prime}=1\right)$ and overcoupled $\left(\beta^{\prime} \gg 1\right)$ cavity. . . . . . . . . . . . . . . 90

4.5 Rectangular cavity modes chart as a function of cavity length. 94

4.6 Total E-field pattern of several $\mathrm{TE}_{m n p}$ and $\mathrm{TM}_{m n p}$ modes inside a rectangular cavity of $22.86 \times 10.16 \times 200 \mathrm{~mm}$ (along $x, y, z)$ dimensions. View of the centered $x z$-plane $\ldots .$.

4.7 Total E-field pattern of the $\mathrm{TE}_{10(15)}$ mode inside the PINE rectangular cavity. View of the centered $x z$-plane $\ldots \ldots .96$

4.8 Cylindrical cavity TE modes chart (9 mm radius). . . . . . 99

4.9 Cylindrical cavity TM modes chart (9 mm radius). . . . . . 99

4.10 Total E-field pattern of several $\mathrm{TE}_{m n p}$ and $\mathrm{TM}_{m n p}$ modes inside a cylindrical $9 \mathrm{~mm}$ radius cavity of $50 \mathrm{~mm}$ length. View of the middle $(\phi=0)$-plane . . . . . . . . . . 100

4.11 Simulation of the E-field distribution in the rectangular PINE cavity performed with CST software . . . . . . . . 102

4.12 MIG Setup (B and C). . . . . . . . . . . . . . . . . . 104

4.13 Calculated frequency deviation of a cavity while a $1.1 \mathrm{~mm}$ radius spherical sample falls through its central axis as function of $\varepsilon^{\prime}$ and $\varepsilon^{\prime \prime} \ldots \ldots \ldots \ldots \ldots \ldots$

4.14 Calculated a) frequency and b) $Q$-factor deviation of a cavity while a water $1.1 \mathrm{~mm}$ radius spherical sample falls through its central axis as function of drop position. . . . . . . . .

4.15 Calculated total absorbed power $P_{i n}$ and power absorbed by a $1.1 \mathrm{~mm}$ water drop $P_{d}$ while falling through the cavity as function of sample position for $P_{g e n}=150 \mathrm{~W}$. . . . . . .

4.16 FDTD simulation of the heating rate for a 1.1 radius water drop using either 12 or $2.45 \mathrm{GHz}$ (incident power $150 \mathrm{~W}$ ).

4.17 Qgen profile $(0.0869<t<0.2199 \mathrm{~s})$ applied to the FDTD thermal simulation of Figure 4.16 (incident power $150 \mathrm{~W}$, drop radius $1.1 \mathrm{~mm}) \ldots \ldots \ldots \ldots$. . . . . . . . . .

4.18 Calculated temperature of a $1.1 \mathrm{~mm}$ radius spherical sample after its fall through the cavity as function of $\varepsilon^{\prime}$ (from 5 to 90) and $\varepsilon^{\prime \prime}$ (from 5 to 50). . . . . . . . . . . . .

5.1 Frequency response of the permittivity in a material due to different polarization mechanisms. $\varepsilon^{\prime}$ represents the dielectric constant and $\varepsilon^{\prime \prime}$ the loss factor. . . . . . . . . . . . 125 
5.2 Experimental setup for the measurement of dielectric properties of a single drop: a) schema and b) photograph. Cavity: $50 \mathrm{~mm}$ length, $9 \mathrm{~mm}$ radius, $4.05 \mathrm{~mm}$ top and bottom holes radius. Drop $2.9 \mathrm{~mm}$ diameter. Temperature $25{ }^{\circ} \mathrm{C}$. . . . . 131

5.3 Coupling measurements: $\left|S_{11}\right|$ modulus as function of iris diameter in $\mathrm{mm}$. Smaller to bigger iris dimensions are presented from right to left. . . . . . . . . . . . . . . 132

5.4 Coupling measurements: $\left|S_{11}\right|$ phase as function of iris diameter in $\mathrm{mm}$. The larger the iris dimensions, the larger the coupling (circumference diameter). . . . . . . . . . .

5.5 QW3D simulation (frontal and transversal view) of the magnitude of the E-Field distribution in a $\mathrm{TM}_{010}$ microwave cylindrical cavity at $10 \mathrm{GHz} . \ldots \ldots \ldots . \ldots \ldots$

5.6 $\mathrm{S}_{11}$ measurement of the $\mathrm{TM}_{010}$ cylindrical microwave cavity at a frequency of $9.998 \mathrm{GHz}$ while 3 consecutive drops are passing through. . . . . . . . . . . . . . . .

$5.7 \mathrm{~S}_{11}$ measurement of the $\mathrm{TM}_{010}$ cylindrical microwave cavity for a single drop at: a) the resonant frequency of the empty cavity $10.054 \mathrm{GHz}$, b) a transient frequency $10.023 \mathrm{GHz}$ between resonances, and c) the resonant frequency of the cavity with the drop 10.005 GHz. . . . . . . . . . . . . . . . . . . 136

$5.8 \mathrm{~S}_{11}$ measurement of the cylindrical microwave cavity as a function of the residence time, over the frequency range of the cylindrical cavity $\mathrm{TM}_{010}$ mode, while consecutive $1.47 \mathrm{~mm}$ radius water drops are passing through. . . . . . . . . . . .

5.9 CST simulation of the cylindrical cavity with a $1.45 \mathrm{~mm}$ radius drop passing through its axis. . . . . . . . . . . 138

5.10 CST simulation of the resonant frequency shift while a $1.45 \mathrm{~mm}$ radius drop is passing through a cylindrical microwave cavity. 139

5.11 Smith chart representation of the reflexion parameter $\mathrm{S}_{11}$. Influence of a drop presence in the center of the cavity on the resonance $\mathrm{TM}_{010}$ mode for different materials. Red marks (circle, square and triangle) correspond to the ones represented in figures 5.7 and 5.12. The triangle is double represented to indicate the relationship with the resonance of the cavity with quartz without drop. . . . . . . . . . . . . 140 
5.12 Magnitude of the $\mathrm{S}_{11}$. Influence of a drop presence in the center of the cavity on the resonance $\mathrm{TM}_{010}$ mode for different materials. Red marks (circle, square and triangle) correspond to the ones represented in figures 5.7 and 5.11. .

5.13 Calculated apparent dielectric properties as a function of assumed water drop radius. . . . . . . . . . . . . . 143

5.14 Calculated apparent dielectric properties as a function of assumed methanol drop radius. . . . . . . . . . . . . . . . 144

5.15 Calculated apparent dielectric properties as a function of assumed methanol drop radius. . . . . . . . . . . . . . . . 144

5.16 Calculated a) frequency and b) $Q$-factor deviation of a cavity while a HMTA cerium based solution $1.1 \mathrm{~mm}$ radius spherical sample falls through its central axis as function of drop position. . . . . . . . . . . . . . . .

5.17 Calculated total absorbed power $P_{i n}$ and power absorbed by a $1.1 \mathrm{~mm}$ HMTA cerium based drop $P_{d}$ while falling through the cavity as function of sample position for $P_{\text {gen }}=150 \mathrm{~W}$.

5.18 FDTD simulation of the heating rate for a 1.1 radius HMTA cerium based drop using a rectangular cavity (incident power $150 \mathrm{~W}) . \ldots \ldots \ldots \ldots \ldots \ldots \ldots$

5.19 Measured temperature of a $1.1 \mathrm{~mm}$ HMTA cerium based drop (thermal properties: $c_{p}=2750.6 \mathrm{~J} \cdot \mathrm{kg}^{-1} \cdot \mathrm{K}^{-1}, \rho=$ $\left.1108.9 \mathrm{~kg} \cdot \mathrm{m}^{-3}, k_{T}=0.6 \mathrm{~W} \cdot \mathrm{m}^{-1} \cdot \mathrm{K}^{-1}\right)$ after its fall through the cavity as function of $P_{i n} \ldots \ldots \ldots$. . . . . . . 148

6.1 MIG Setup (B and C). . . . . . . . . . . . . 155

6.2 Cylindrical cavity: a) CST cavity simulation, b) ITACA cavity manufactured for low power measurements and c) PSI cavity manufactured with option of high power measurements. 157

6.3 Simulation of the a) E-field distribution in a rectangular cavity and b) interaction of the field with a drop performed with CST software. . . . . . . . . . . . . . . 158

6.4 ITACA rectangular cavity manufactured for low and high power measurements: a) Cavity in the MIG system, b) iris, c) waveguide connexion. . . . . . . . . . . . . 158

6.5 Microwave Generator. . . . . . . . . . . . . . . . 159

6.6 Amplifier. . . . . . . . . . . . . . . . . . 160

6.7 Isolator. . . . . . . . . . . . . . . . . . 162 
6.8 Waveguides: a) transition from WRD750D24 to WR90; b) directional coupler in WR90; c) 90 degree twist in WRD750D24;

d) 90 degree twist in WR90. . . . . . . . . . . . . . 163

6.9 a) Adapter from WR90 to N. b) Detector . . . . . . . . . 164

6.10 Vector Network Analysers. . . . . . . . . . . . . . . . 166

6.11 Oscilloscope. . . . . . . . . . . . . . . . 166

6.12 CST rectangular empty cavity frequency response. . . . . . 170

6.13 Coupling measurements on rectangular cavity: $\left|S_{11}\right|$ module depending on the coupling dictated by the insertion of the screw. The deeper the screw, the stronger the resonance and the higher its frequency shift. . . . . . . . . . . . . . 171

6.14 Coupling measurements on rectangular cavity: $\left|S_{11}\right|$ phase depending on the coupling dictated by the insertion of the screw. The deeper the screw, the smaller the circumference on the Smith chart. . . . . . . . . . . . . . . . . 171

6.15 Detector characterization curve. . . . . . . . . . . . 172

6.16 Input power at amplifier vs. input power going to the cavity (measured with the detector). . . . . . . . . . 173

6.17 MIG system assembly in the Hotlab of the Paul Scherrer Institut. . . . . . . . . . . . . . . . . 173

6.18 Schema broth preparation, generation of drops and microwave heating. . . . . . . . . . . . . . 175

6.19 Schema of the heating experiment protocol. . . . . . . . . 176

6.20 Thermal captures of water drops with ThermaCAM FLIR E45. . . . . . . . . . . . . . . . . . 177

6.21 Temperature of a $1.1 \mathrm{~mm}$ water drop (thermal properties: $\left.c_{p}=4190 \mathrm{~J} \cdot \mathrm{kg}^{-1} \cdot \mathrm{K}^{-1}, \rho=1000 \mathrm{~kg} \cdot \mathrm{m}^{-3}, k_{T}=0.6 \mathrm{~W} \cdot \mathrm{m}^{-1} \cdot \mathrm{K}^{-1}\right)$ after its fall through the cavity as function of $P_{i n}$, for two different modes. . . . . . . . . . . . . . . . . 178

6.22 Temperature of a $1.1 \mathrm{~mm}$ water drop after its fall through the cavity as function of $P_{i n} \ldots \ldots \ldots \ldots 178$

6.23 Cerium gelled spheres collected in water. . . . . . . . . 179

B.1 Crank Nicholson scheme for a one dimensional approach. . . 206

D.1 Bessel function of 1 st order $J_{m}(x)$ for $m=0,1,2 \ldots \ldots .225$ 


\section{List of symbols}

\section{Mathematical formulation}

$\begin{array}{ll}\mathbf{X} & \text { Matrix } \\ \mathbf{x} & \text { Vector } \\ x, X & \text { Scalar } \\ X_{i, j} & i, j \text { component of matrix } \mathbf{X} \\ \mathbf{x}^{n} & \text { Vector } \mathbf{x} \text { in } n \text {-th time instant } \\ x_{i}^{n}, X_{i}^{n} & i \text {-th component of vector } \mathbf{x}^{n} \\ \mathbf{I}_{N \times N} & \text { Identity matrix of size } N \times N \\ |\cdot| & \text { Absolute value } \\ n & \text { Time index } \\ i & \text { Space index } \\ t & \text { Discrete time } \\ j & \text { Complex number, } j^{2}=-1 \\ \Delta x & \text { Discrete interval of x variable } \\ N_{x} & \text { Number of points in } x \\ \nabla & \text { Vector differential operator }\end{array}$

\section{Manuscript nomenclature}

Thermal Related nomenclature

$\begin{array}{ll}\alpha & \text { Thermal diffusivity }\left(\mathrm{m}^{2} \cdot \mathrm{s}^{-1}\right) \\ \beta & \text { Volumetric heat capacity }\left(\mathrm{J} \cdot \mathrm{m}^{-3} \cdot \mathrm{K}^{-1}\right) \\ c_{p} & \text { Specific heat capacity at constant pressure }\left(\mathrm{J} \cdot \mathrm{kg}^{-1} \cdot \mathrm{K}^{-1}\right) \\ D & \text { Characteristic length, drop diameter }(\mathrm{m}) \\ \Delta x, \Delta z, \Delta r & \text { Spatial sampling period }(\mathrm{m}) \\ \Delta t & \text { Temporal sampling period }(\mathrm{s}) \\ \epsilon & \text { Emissivity }(-) \\ \varepsilon_{0} & \text { Vacuum permittivity }\left(\mathrm{F} \cdot \mathrm{m}^{-1}\right) \\ \varepsilon^{\prime} & \text { Dielectric constant }(-) \\ \varepsilon^{\prime \prime} & \text { Loss Factor }(-) \\ E_{r m s} & \text { Root mean square of } \mathrm{E}-\text { field }\left(\mathrm{V} \cdot \mathrm{m}^{-1}\right) \\ \eta & \text { Dynamic viscosity }\left(\mathrm{kg} \cdot \mathrm{m}^{-1} \cdot \mathrm{s}^{-1}\right)\end{array}$




\begin{tabular}{|c|c|}
\hline$f_{0}$ & Resonant frequency $(\mathrm{Hz})$ \\
\hline$H$ & Enthalpy $\left(\mathrm{J} \cdot \mathrm{m}^{-3}\right)$ \\
\hline$\dot{H}$ & Rate of thermal energy change $\left(\mathrm{W} \cdot \mathrm{m}^{-3}\right)$ \\
\hline$h_{T}$ & Convective heat transfer coefficient $\left(\mathrm{W} \cdot \mathrm{m}^{-2} \cdot \mathrm{K}^{-1}\right)$ \\
\hline$k_{T}$ & Thermal conductivity $\left(\mathrm{W} \cdot \mathrm{m}^{-1} \cdot \mathrm{K}^{-1}\right)$ \\
\hline$L$ & Length of the material $(\mathrm{m})$ \\
\hline$N_{u}$ & Nusselt number (-) \\
\hline$\nu$ & Kinematic viscosity $\left(\mathrm{m}^{2} \cdot \mathrm{s}^{-1}\right)$ \\
\hline$P_{r}$ & Prandtl number $(-)$ \\
\hline$Q$ & Heat transfer rate $(\mathrm{W})$ \\
\hline$Q_{\text {flux }}$ & $\begin{array}{l}\text { Heat flux: heat transfer rate per unit area normal to the } \\
\text { direction of heat flow }\left(\mathrm{W} \cdot \mathrm{m}^{-2}\right)\end{array}$ \\
\hline $\mathbf{q}_{\text {flux }}$ & Heat flux vector \\
\hline$Q_{g e n}$ & Microwaves heat generation rate $\left(\mathrm{W} \cdot \mathrm{m}^{-3}\right)$ \\
\hline$Q_{h_{T}}$ & Convection heat exchange $(\mathrm{W})$ \\
\hline$Q_{\text {local }}$ & $\begin{array}{l}\text { Heat rate generated by a local or chemical reaction } \\
\left(\mathrm{W} \cdot \mathrm{m}^{-3}\right)\end{array}$ \\
\hline$Q_{\text {loss }}$ & $\begin{array}{l}\text { Loss rate referred to the surface convection and } \\
\text { radiation of energy }\left(\mathrm{W} \cdot \mathrm{m}^{-3}\right)\end{array}$ \\
\hline$Q_{n e t}$ & Net rate of heat generation in a material volume $\left(\mathrm{W} \cdot \mathrm{m}^{-3}\right)$ \\
\hline$Q_{p h}$ & $\begin{array}{l}\text { Heat rate derivated from phase changes or material } \\
\text { transformation }\left(\mathrm{W} \cdot \mathrm{m}^{-3}\right)\end{array}$ \\
\hline$Q_{\text {rad }}$ & Radiation heat exchange $(\mathrm{W})$ \\
\hline$R, r$ & Radius of a sphere or cylinder (m) \\
\hline$R_{e}$ & Reynolds number $(-)$ \\
\hline$\rho$ & Material density $\left(\mathrm{kg} \cdot \mathrm{m}^{-3}\right)$ \\
\hline$S$ & Surface $\left(\mathrm{m}^{2}\right)$ \\
\hline$\sigma$ & Stefan-Boltzmann constant $\left(\mathrm{W} \cdot \mathrm{m}^{-2} \cdot \mathrm{K}^{-4}\right)$ \\
\hline$t$ & Continuous time (s) \\
\hline$t_{f}$ & Final time $(\mathrm{s})$ \\
\hline$t_{i}$ & Initial time $(\mathrm{s})$ \\
\hline $\mathrm{t}$ & Vector temperature \\
\hline$T_{K}$ & Kelvin temperature $(\mathrm{K})$ \\
\hline$T_{C}$ & Celsius temperature $\left({ }^{\circ} \mathrm{C}\right)$ \\
\hline$T_{F}$ & Fahrenheit temperature $\left({ }^{\circ} \mathrm{F}\right)$ \\
\hline$T_{\infty}$ & Environmental external temperature (K) \\
\hline$T_{S}$ & Surface temperature $(\mathrm{K})$ \\
\hline$T^{*}$ & Dimensionless temperature $(-)$ \\
\hline
\end{tabular}




$\begin{array}{ll}u & \text { Velocity }\left(\mathrm{m} \cdot \mathrm{s}^{-1}\right) \\ \omega & \text { Angular frequency }\left(\mathrm{rad} \cdot \mathrm{s}^{-1}\right) \\ x^{*} & \text { Dimensionless length }(-)\end{array}$

\section{MicrowaVes RELATED NOMENClATURE}

a Rectangular cavity width $(\mathrm{m})$

$\alpha_{z} \quad$ Attenuation constant $\left(\mathrm{Np} \cdot \mathrm{m}^{-1}\right)$

$A_{m n}, B_{m n} \quad$ Constants as function of the incident power (-)

$A^{\prime}, B^{\prime} \quad$ Constants related with $P_{d}$ calculation (-)

$b \quad$ Rectangular cavity high $(\mathrm{m})$

$\beta_{x} \quad$ Phase constant in $x$ direction $\left(\mathrm{rad} \cdot \mathrm{m}^{-1}\right)$

$\beta_{y} \quad$ Phase constant in $y$ direction $\left(\mathrm{rad} \cdot \mathrm{m}^{-1}\right)$

$\beta_{z} \quad$ Phase constant in $z$ direction $\left(\mathrm{rad} \cdot \mathrm{m}^{-1}\right)$

$\varepsilon_{0} \quad$ Vacuum permittivity $\left(\mathrm{F} \cdot \mathrm{m}^{-1}\right)$

$\varepsilon^{*} \quad$ Complex relative permittivity $\left(\mathrm{F} \cdot \mathrm{m}^{-1}\right)$

$\varepsilon_{r} \quad$ Relative dielectric constant $(-)$

$\varepsilon^{\prime} \quad$ Dielectric constant (-)

$\varepsilon^{\prime \prime} \quad$ Loss Factor $(-)$

$\epsilon_{m}, \epsilon_{n}, \epsilon_{p} \quad$ Constants related with energies and powers calculation (-)

$\vec{E} \quad$ Electric field inside a cavity $\left(\mathrm{V} \cdot \mathrm{m}^{-1}\right)$

$E_{r m s} \quad$ Root mean square of E-field $\left(\mathrm{V} \cdot \mathrm{m}^{-1}\right)$

$\eta \quad$ Filling factor in perturbation model $(-)$

$\eta^{\prime} \quad$ Characteristic impedance for a TEM wave in free space $(\Omega)$

$f \quad$ Frequency $(\mathrm{Hz})$

$f_{\text {gen }} \quad$ Working frequency of the microwave generator $(\mathrm{Hz})$

$f_{u} \quad$ Unloaded resonant frequency of a cavity $(\mathrm{Hz})$

$f_{u 0} \quad$ Unloaded resonant frequency of an empty cavity $(\mathrm{Hz})$

$f_{u 0, m n p} \quad$ Unloaded resonant frequency of an empty cavity

for a $\mathrm{TE}_{m n p}$ or $\mathrm{TM}_{m n p}$ mode $(\mathrm{Hz})$

$\gamma_{z} \quad$ Propagation constant $\left(\mathrm{rad} \cdot \mathrm{m}^{-1}\right)$

$h \quad$ Cylindrical cavity length $(\mathrm{m})$

$\vec{H} \quad$ Magnetic field inside a cavity $\left(\mathrm{A} \cdot \mathrm{m}^{-1}\right)$

$\vec{H}_{t a n} \quad$ Tangential magnetic field to the cavity walls $\left(\mathrm{A} \cdot \mathrm{m}^{-1}\right)$

$J_{m}(x) \quad$ Bessel function of the first kind (-)

$k \quad$ Propagation constant in free space $\left(\mathrm{rad} \cdot \mathrm{m}^{-1}\right)$

$k_{0} \quad$ Propagation constant in an empty cavity $\left(\mathrm{rad} \cdot \mathrm{m}^{-1}\right)$

$k_{c, m n} \quad$ Cuttoff wave number $\left(\mathrm{rad} \cdot \mathrm{m}^{-1}\right)$

$L \quad$ Cavity length $(\mathrm{m})$ 


$\begin{array}{ll}\lambda & \text { Wavelength }(\mathrm{m}) \\ \mu_{0} & \text { Vacuum permeability }\left(\mathrm{V} \cdot \mathrm{s} \cdot \mathrm{A}^{-1} \cdot \mathrm{m}^{-1}\right) \\ \omega & \text { Angular frequency }\left(\mathrm{rad} \cdot \mathrm{s}^{-1}\right) \\ \omega_{u} & \text { Angular unloaded resonant frequency of a cavity }\left(\mathrm{rad} \cdot \mathrm{s}^{-1}\right) \\ \omega_{u 0} & \text { Angular unloaded resonant frequency of an empty } \\ & \text { cavity }\left(\mathrm{rad} \cdot \mathrm{s}^{-1}\right) \\ \Omega & \text { Complex angular frequency }\left(\mathrm{rad} \cdot \mathrm{s}^{-1}\right) \\ P_{0} & \text { Real power dissipated by the cavity walls }(\mathrm{W}) \\ P_{d} & \text { Real power dissipated by the dielectric of a cavity }(\mathrm{W}) \\ P_{e x} & \text { Dissipated power by the external circuit coupled of a cavity }(\mathrm{W}) \\ P_{i n} & \text { Total real power dissipated inside a cavity }(\mathrm{W}) \\ P_{m n} & \text { Zeros of Bessel function }(-) \\ P_{m n}^{\prime} & \text { Zeros of the derivative of Bessel function }(-) \\ Q_{c} & \text { Quality }(Q) \text { factor due to the walls cavity generated losses }(-) \\ Q_{c 0} & Q \text {-factor due to the walls empty cavity generated losses }(-) \\ Q_{d} & Q \text {-factor due to the dielectric cavity generated losses }(-) \\ Q_{e} & \text { External } Q \text {-factor }(-) \\ Q_{u} & \text { Unloaded } Q \text {-factor of a cavity }(-) \\ Q_{u 0} & \text { Unloaded } Q \text {-factor of an empty cavity }(-) \\ R_{s} & \text { Surface resistance of a conductor }(\Omega) \\ R, r & \text { Radius sphere or cylinder }(\mathrm{m}) \\ \rho & \text { Reflection factor } \\ S_{c} & \text { Cavity superficial contour }\left(\mathrm{m}^{2}\right) \\ \sigma & \text { Metal conductivity }\left(\mathrm{S} \cdot \mathrm{m}^{-1}\right) \\ \sigma_{e} & \text { Equivalent dielectric conductivity }\left(\mathrm{S} \cdot \mathrm{m}^{-1}\right) \\ \tan \delta & \text { Loss tangent }(-) \\ T E_{m n p} & \text { Transversal Electric } m n p \text { resonant mode in } z \text { direction } \\ T M_{m n p} & \text { Transversal Magnetic } m n p \text { resonant mode in } z \text { direction } \\ V_{c} & \text { Cavity volume }\left(\mathrm{m}^{3}\right) \\ v_{s} & \text { Sample volume }\left(\mathrm{m}^{3}\right) \\ W_{e} & \text { Medium stored electric energy inside a cavity }(\mathrm{J}) \\ W_{m} & \text { Medium stored magnetic energy inside a cavity }(\mathrm{J}) \\ X_{s} & \text { Surface reactance of a conductor }(\Omega) \\ Z_{s} & \text { Surface impedance of a conductor }(\Omega) \\ Z_{T E} & \text { Characteristic impedance of TE guided modes }(\Omega) \\ Z_{T M} & \text { Characteristic impedance of TM guided modes }(\Omega) \\ & \\ & \end{array}$




\section{Abbreviations and Acronyms}

$\begin{array}{ll}\text { ADS } & \text { Accelerator Driven System } \\ \text { a.k.a. } & \text { also known as } \\ \text { BC } & \text { Boundary Condition } \\ \text { BWR } & \text { Boiling Water Reactor } \\ \text { CCEM } & \text { Competence Center Energy and Mobility } \\ \text { DES } & \text { Dielectric Spectroscopy } \\ \text { e.g. } & \text { for example (from the latin exempli gratia) } \\ \text { ETHZ } & \text { Eidgenössische Technische Hochschule Zürich } \\ \text { EM } & \text { Electromagnetic } \\ \text { EMPA } & \text { Eidgenössische Materialprfungs- und Forschungsanstalt } \\ \text { FBR } & \text { Fast Breeder Reactor } \\ \text { FD } & \text { Finite Difference } \\ \text { FDTD } & \text { Finite Difference Time Domain } \\ \text { FEM } & \text { Finite Elements Method } \\ \text { GAM } & \text { Generalized Admittance Matrix } \\ \text { GSM } & \text { Generalized Scattering Matrix } \\ \text { GUI } & \text { Graphical User Interface } \\ \text { HLW } & \text { High Level Waste } \\ \text { HTGR } & \text { High Temperature Gas cooled Reactors } \\ \text { HMTA } & \text { Hexamethylenetetramine } \\ \text { HWR } & \text { Heavy Water Reactor } \\ \text { IAEA } & \text { International Atomic Energy Agency } \\ \text { ICRP } & \text { International Commission on Radiological Protection } \\ \text { i.e. } & \text { that is (from the latin id est) } \\ \text { ILW } & \text { Intermediate Level Waste } \\ \text { ISL } & \text { In Situ Leaching } \\ \text { ISR } & \text { In Situ Recovering } \\ \text { ITACA } & \text { Institute for the Applications of Advanced Information } \\ & \text { and Communication Technologies } \\ \text { LLW } & \text { Low Level Waste } \\ \text { LWR } & \text { Light Water Reactor } \\ \text { MA } & \text { Minor Actinides } \\ \text { MIG } & \text { Microwave Internal Gelation } \\ & \end{array}$




$\begin{array}{ll}\text { MOX } & \text { Mixed Oxide } \\ \text { NEA } & \text { Nuclear Energy Agency } \\ \text { OECD } & \text { Organisation for Economic Co-operation and Development } \\ \text { Pa } & \text { Protactinium } \\ \text { PDE } & \text { Partial Differential Equation } \\ \text { PHWR } & \text { Pressurized Heavy Water Reactor } \\ \text { PINE } & \text { Platform for Innovative Nuclear FuEls } \\ \text { PSI } & \text { Paul Scherrer Institut } \\ \text { Pu } & \text { Plutonium } \\ \text { PWR } & \text { Pressurized Water Reactor } \\ \text { P\&T } & \text { Partitioning and Transmutation } \\ \text { QS } & \text { Quasi Static } \\ \text { QW3D } & \text { QuickWave 3D Simulator } \\ \text { SCWR } & \text { Supercritical Water Reactor } \\ \text { SFR } & \text { Sodium Fast Reactor } \\ \text { SP } & \text { Sphere-Pac } \\ \text { TE } & \text { Transversal Electric } \\ \text { TEM } & \text { Transversal Electromagnetic } \\ \text { Th } & \text { Thorium } \\ \text { TM } & \text { Transversal Magnetic } \\ \text { TRU } & \text { Transuranic } \\ \text { U } & \text { Uranium } \\ \text { UDEPO } & \text { World Distribution of Uranium Deposits } \\ \text { UPV } & \text { Universitat Politècnica de València } \\ \text { VNA } & \text { Vector Network Analyser } \\ \text { WR } & \text { Rectangular Waveguide } \\ \text { WRD } & \text { Double Ridge Waveguide }\end{array}$




\section{Introduction and Scope}

\subsection{Background}

Since the Discovery of the Radioactivity by Antoine Henri Becquerel, who exposed a photographic plate to uranium salts in 1896, a continuous research with radioactive materials has been conducted allowing the development of nuclear energy.

From the three common Uranium isotopes ${ }^{238} \mathrm{U},{ }^{235} \mathrm{U}$ and ${ }^{234} \mathrm{U},{ }^{235} \mathrm{U}$ is the only naturally occurring fissile isotope, having higher fission crosssection for slow neutrons. ${ }^{235} \mathrm{U}$ is therefore the essential component of nuclear fuel and has to be enriched up to $5 \%$ before the fuel manufacture [1]. However only $0.7198-0.7202 \%$ of the Uranium isotopes are ${ }^{235} \mathrm{U}$ being the majority of the isotopes $(99.2739-99.2752 \%){ }^{238} \mathrm{U}$, which has a small probability for spontaneous fission. Moreover, after its reactor utilisation e.g. in a Light Water Reactor (LWR), the ${ }^{235} \mathrm{U}$ is again an insignificant part of the spent nuclear fuel composition [2], which contains additional components such as ${ }^{239} \mathrm{Pu}$ and Minor Actinides (MA) with high radiotoxicity. 
The radio-toxicity of the disposed LWR spent fuel takes about 100000 years of natural decay to reach a level comparable to that of the natural uranium ore. An advanced nuclear fuel cycle can effectively reduce this period to 1000 years. To achieve this reduction the LWR spent fuel has to be reprocessed by means of a chemical separation, which separates uranium, plutonium and the MAs for an advanced MA containing Mixed Oxides (MA-MOX) fuel fabrication [3] used in (Sodium) Fast Reactors (SFR) in a closed cycle or once trough in an Accelerator Driven System (ADS) [4].

Although fuel pressed in form of pellets and filled into fuel pins is a consolidated standard, there is a increasing interest in emerging fuel kernels e.g. Sphere-Pac (SP) fuel, which has a simpler fabrication and offers several advantages when the fuel is coming from reprocessing. One of the most important advantages is that many mechanical devices like presses and grinding machines can be avoided, decreasing the maintenance requirements $[5,6]$. This benefit must be taken into account because, compared to fresh fuel, the radiation dose of spent fuel becomes very important (minor actinides), making the operation in hot-cells inevitable.

Internal gelation is used to produce SP fuel because it is an aqueous chemical process and almost powder-less [7-10]. With the internal gelation, the contamination of the equipment is minimized. This allows a more continuous operation with less cleaning operations and lowers the human radiation exposure and contamination risk. The key parameter in this chemical process is no longer the mixing of the catalyst but is the ability to control the heating in the volume of the solution either by contact or contact-less.

The most common procedure to shape the nuclear fuel in spheres is the internal gelation by contact with silicon oil, developed by the KEMA laboratory in the 1960s [8]. For this purpose the cooled (at roughly 270$272 \mathrm{~K}$ ) feed solution is dropped into the hot (roughly 373-379 K) silicon oil. Because of the immersion into the oil (former procedure), the spheres have to be cleaned subsequently using an organic solvent and ammonia. Depending on the development stage of the equipment, this can presuppose sophisticated recycling equipment, which is an important drawback of the process. However, nowadays the silicon oil based process is still used in research [11].

By using contact-less volumetric microwave heating as heat source, a large amount of liquid waste can be avoided and, as seen in the flowchart 


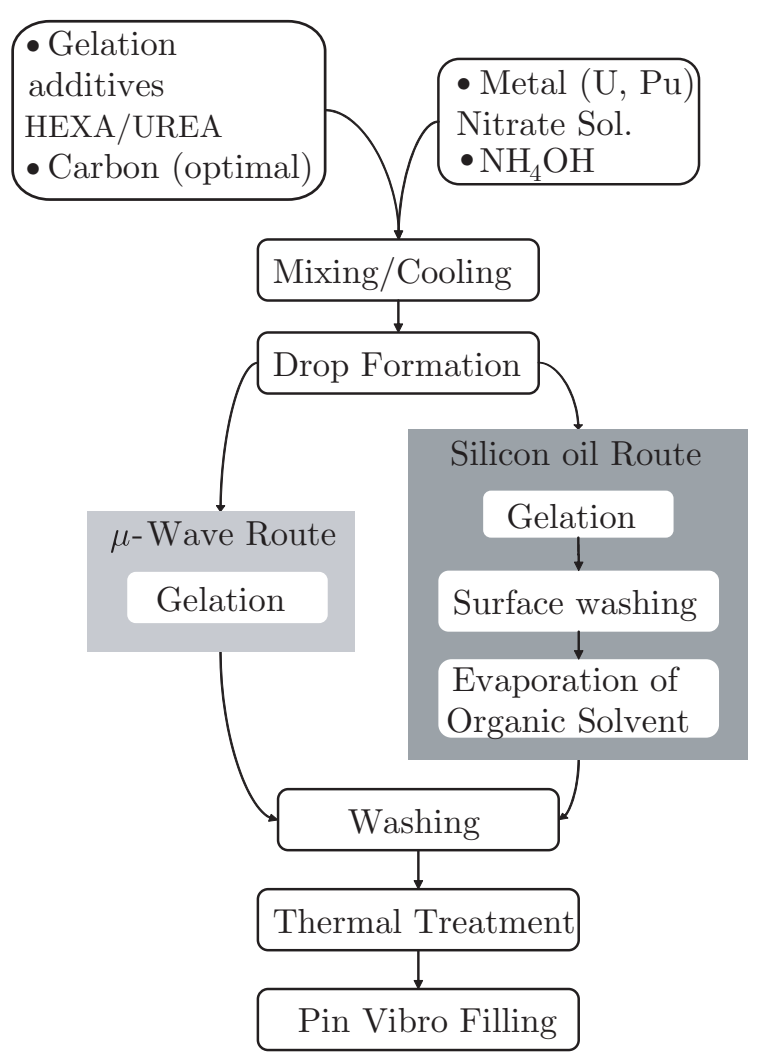

Figure 1.1. Comparative flowchart of contact (by silicon oil) and contact-less (through microwaves) internal gelation.

represented in Figure 1.1, the production unit is much simplified (about half of the space and parts). The flowchart represents an overview of the steps that are involved in the production of SP fuel [7]: first the components are mixed together to form the feed solution and the mixture is cooled to avoid a premature gelation before it is dropped in the heat medium; after the gelation the spheres are washed and enter in a thermal treatment to be solidified and finally filled into the pin.

Microwave heating has important advantages over conventional techniques, which are described below.

- Uniform heating within the precursor leads to high chemical and 
micro-structural homogeneity as well as to finer micro- and nanostructures and thus to improved physical, chemical and mechanical properties.

- Rapid processing heating/cooling rates allow short processing times, important energy savings and high-throughput processing, enable the restraint of metastable phases and, most important, of ultra-fine or nano-scale structures.

- Ability for selective heating and on the potential to modify solidstate and chemical reaction pathways, to yield new materials, phases or micro-structures.

Currently, microwaves are used in heating or sintering processes of a wide range of materials, including metallic powders. Common applications range from food conditioning, to wood processing, sterilization and drying, to the non hazardous chemical synthesis of materials. Increasing attention is recently being paid to the use of microwaves in high-temperature applications, in particular for metals processing.

The idea of Microwave Internal Gelation (MIG) has already been partially investigated [12-18]. Knotik [12] and Lederberger [13, 14] were working in the $8-14 \mathrm{GHz}$ regime, whereas Yamagashi [15-17] and Rosin [18] used the commercially widely applied $2.45 \mathrm{GHz}$ frequency.

Around $12 \mathrm{GHz}$, the applicators were upright mono-mode cavities, where several E-field maxima are established. The required microwave power levels were $80-100 \mathrm{~W}$, resulting in a temperature increase of $60-80$ $\mathrm{K}$. This was sufficient to achieve adequate gelation. Using $2.45 \mathrm{GHz}$, Yamagishi $[15-17]$ required $5000 \mathrm{~W}$ to achieve the same temperature increase of $80 \mathrm{~K}$. The decrease of energy transfer efficiency when decreasing the frequency is due to the lowering of the quantum energy and some scattering influence [19]. Rosin [18] experimented some optimization of the cavity design and pre-heating of the droplets at the nozzle. When reviewing these studies, the use of the $8-14 \mathrm{GHz}$, with the about $1-2$ orders of magnitude higher energy transfer efficiency [18], seems to be essential for a successful microwave gelation, although the generators and amplifiers are much more expensive for the $10-12 \mathrm{GHz}$ range. When applied to an industrial scale, these factors become much less grave, and the (energy) efficient operation of a plant becomes more important. 
The literature [19-24] shows that the microwave internal gelation depends on several parameters: the frequency, the power level, the time of residence of the material (droplets) inside the cavity, the properties of the material (thermal and dielectric properties), how the field interacts with the drops, the boundary conditions, the electromagnetic fields distribution of the microwave mode and the microwave cavity model (shape) used for this purpose. Each of these parameters is studied individually and unified, however some options e.g. cylindrical cavities, have not been evaluated.

Currently, there is no available information of other research groups working on microwave internal gelation; the experiments described in [18] are the last documented activities and the usage of $2.45 \mathrm{GHz}$ is possibly one of the main reasons for some attempts to fail, as the energy transfer is very unfavourable at this frequency, for the short residence time of the dielectric material inside the cavity. In X Band (between $8.2 \mathrm{GHz}$ and 12.4 $\mathrm{GHz}$ ) the energy transfer is maximized in such a short falling time of the drops and the smaller dimensions of the components are also advantageous.

\subsection{Motivation}

The main motivation of this thesis is the development of an advanced production method for Sphere-Pac fuel, in which the internal gelation is triggered by applying high frequency electromagnetic waves. This is also one of the main goals of the funded project Platform for Innovative Nuclear FuEls (PINE), which was supported by the Competence Center Energy and Mobility (CCEM) from Switzerland and was carried out in the Paul Scherrer Institut (PSI) from Switzerland with the collaboration of the Universitat Politècnica de València (UPV) from Spain, the Eidgenössische Technische Hochschule Zürich (ETHZ) and the EMPA Thun Materials Science \& Technology as the main partners in Switzerland.

The new SP fuel concept for SFR and ADS comes from a chemical reprocessing of the LWR spent fuel and therefore contains minor actinides. Compared to fresh fuel, the radiation dose gains high importance and accordingly the treatment in hot-cells is inevitable. Moreover the use of this fuel cuts down the decay times of the nuclear waste from 100000 years to 1000 years during its underground disposal.

When producing SP fuel instead of the classical fuel pellets, many me- 
chanical devices, e.g. presses and grinding machines, can be avoided. This importantly decreases the maintenance requirements. Furthermore the proposed internal gelation is almost powder-less. Therefore the contamination of the equipment is minimized allowing a more continuous operation with less cleaning operations and lowering the human radiation exposure and contamination risks.

On the other hand, by using microwaves as a heating source a large amount of liquid waste (compared to the formally used silicon oil based technique) can be avoided and the production unit is simplified.

Nowadays no industrial production of SP fuel exists. Since the advantages of SP are of increasing interest and recognized by the international community the Microwave internal gelation discussed in this dissertation can be a solution for a big scale production.

\subsection{Scope of the Thesis}

The main scope of the proposed thesis is as follows:

To develop new modelling and experimental methods for the understanding of the interaction between the electromagnetic field distribution and the materials during the microwave internal gelation of aqueous droplets. To design and manufacture a microwave heating cavity, of which the main purpose is the internal gelation of nuclear fuel after a previous phase dealing with the production of inactive materials (ceria and natural uranium based microspheres). To optimize the microwave applicator integrating the boundary processes as the gelation chemistry and the drop formation, before applicability at laboratory and later industrial scale can be reached.

Some particular tasks and aims from the main scope are the following:

- To study and analyse the physical and chemical phenomena that govern the reaction to form the nuclear fuel by microwave internal gelation.

- To explore the main problems appearing in this application context and consequently propose new solutions.

- To develop a model for microwave heating of materials (dielectric and metallic) able to determine the temperature of a material (droplets 
in particular) as a function of the frequency, materials properties (dielectric and thermal) and energy (power and time).

- To design, manufacture and optimize cavities for the internal gelation use.

- To validate the results of the developed theoretical models with the measurements of the manufactured cavities, proving at the same time their functionality.

\subsection{Organization of the Thesis}

The following chapters of this manuscript describe the research that has been undertaken to reach the aforementioned goals. Figure 1.2 shows a block diagram with the organization and connections of the chapters which are presented as follows:

- Chapter 2: This first part of the manuscript contains the state of the art research related on one side to nuclear energy and fuels and on the other side to microwave internal gelation. The first sections describe the nuclear fuels from their extraction to their recycling after usage in a closed fuel cycle, mentioning the hazards that motivate the research of new recycling mechanisms. Then, the Sphere-Pac fuel production is introduced in all its modalities. Finally, some preliminaries related to the internal gelation by microwaves and an introduction to the PINE project are presented.

- Chapter 3: Here, a the thermal FDTD model used to evaluate the heating of a material is developed. First, the parameters that influence the behaviour of the model are described, giving special attention to the boundary conditions which surround the material. The FDTD solution of the model is later given and implemented. Furthermore, several examples are executed to highlight the parameter influences and the model is then validated by other methods.

- Chapter 4: Microwave cavities and their involvement in the PINE project are described. First, the cavity functionalities (generation of fields depending on the working mode, energy and power) and 


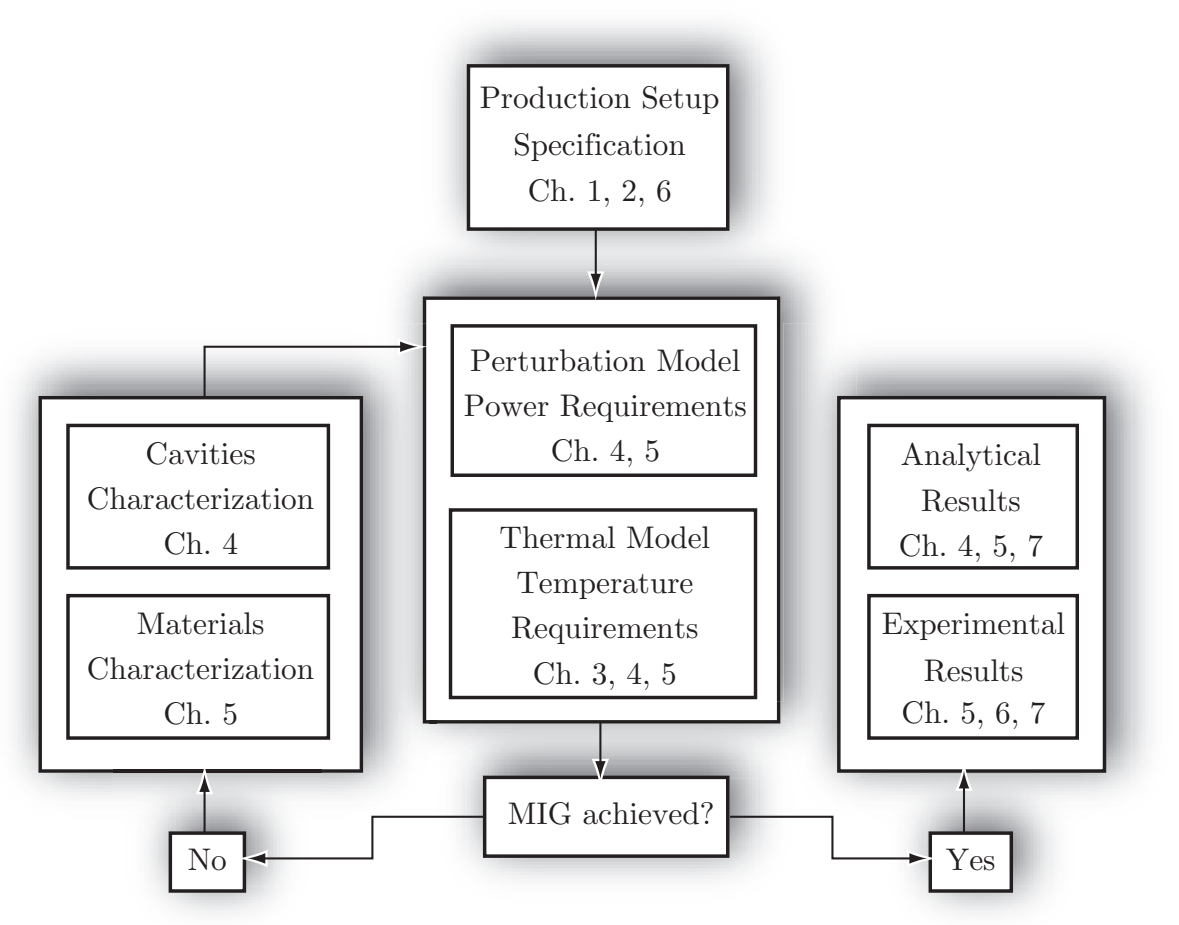

Figure 1.2. Block diagram of the Microwave Internal Gelation process with the corresponding manuscript chapters.

their modifications due to the dielectrics and coupling are presented. Next, specific designs as a rectangular cavity is further introduced in the PINE project. Taking into account the geometry of the samples that are heated inside, the perturbation method, which analyses the interaction of the fields produced in the cavity with the samples and the dissipated powers in both, is presented. Finally, the thermal model of Chapter 3 is updated and results are shown.

- Chapter 5: The importance of the dielectric properties of the materials that are going to be heated in a microwave cavity are introduced. First, the concept of the dielectric properties and several techniques used for their measurement are described. Furthermore, a new characterization method for measuring the dielectric properties of mate- 
rials in form of drops which freely fall through a microwave cavity is presented and proved. In the last part, the perturbation and thermal models presented in Chapters 3 and 4 are updated showing the new results.

- Chapter 6: The MIG system structure and its functionality as a fuel producer are presented. First, the schemas of the system and all the necessary equipment are described. Then, a power study is done to ensure the minimal needed power while preventing the system from failure. Furthermore, temperature measurements are carried out ensuring the minimal temperature increase for the internal gelation. Finally, experiments with inactive metal surrogate solution samples are performed and gelated spheres are successfully produced.

- Chapter 7: Finally, conclusions obtained through this thesis are emphasized, including some guidelines for future research lines. A list of published works related to this thesis is also given. 



\section{Preliminaries and State of the Art}

2.1 Introduction . . . . . . . . . . . . 13

2.2 Nuclear Fuel Cycle . . . . . . . . . . . . . . . . . . . . . 14

2.3 Solid Nuclear Fuel . . . . . . . . . . . . . . . . . . . 23

2.4 Sphere-Pac Fuel Production . . . . . . . . . . . . . . 27

2.5 State of the Art of the Internal Gelation SP Fuel Production 30

2.6 Conclusions . . . . . . . . . . . . . . . . . . 35 



\section{Preliminaries and State of the Art}

IN THIS CHAPTER, the global framework for the nuclear fuel cycle is presented, with an emphasis on the fuel production techniques including the Sphere-Pac fuel. The previous research in the different production techniques is also described as a starting point of the research work for this thesis.

\subsection{Introduction}

The prevision of a coal and oil shortage, used in the fossil fuel power generation, introduced the uranium as a fission product in the thermal power plants to produce energy. The main drawback of uranium based fuel is its increasing radioactivity and toxicity after its use. The spent nuclear fuel is planned to be disposed underground for a long term until its radiation level naturally decreases to the one of the natural uranium ore. However, that fuel can be reprocessed and further reused in a way to decrease the decay times of the disposal.

The general framework for nuclear fuel cycle presented in this chapter has been mostly inspired by excellent overviews on the topic, especially the ones published by the Nuclear Energy Agency (NEA) [3, 25] and the 
International Atomic Energy Agency (IAEA) [26-31].

\subsection{Nuclear Fuel Cycle}

Before using the uranium for power generation it has to be processed from its natural ore state (mined from the ground). The uranium fuel cycle is described as follows:

1. Exploration and evaluation of the uranium deposits to extract the materials.

2. Mining and milling to get the yellow cake i.e. concentrated uranium.

3. Refining and conversion of the concentrated uranium into either uranium dioxide $\left(\mathrm{UO}_{2}\right)$ for Heavy Water Reactors (HWR) or gaseous uranium hexafluoride $\left(\mathrm{UF}_{6}\right)$, which needs further procedure to get enriched $\mathrm{UO}_{2}$ for Light Water Reactors (LWR).

4. Enrichment to increase the proportion of the fissile uranium, ${ }^{235} \mathrm{U}$.

5. Fuel fabrication in the form of pellets.

6. Electricity generation thanks to the nuclear reactions effected in the reactors.

7. Reprocessing of the residual uranium after a storage period.

8. Disposal until the residual uranium recovers the natural radioactivity levels of the ore.

Figure 2.1 shows an overview of a complete nuclear fuel cycle following the above mentioned sequence. Inside this cycle two main sub-cycles are differentiated: on one side the LWR fuel cycle where the reactors need ${ }^{235} \mathrm{U}$ enriched fuel to produce energy and on the other side the advanced reactor fuel cycle, that treats the spent fuel with the main purpose to cut down the decay times of the radioactive waste before its disposal and to utilize the fertile ${ }^{238} \mathrm{U}$.

The advanced fuel cycle could be a fully closed cycle when the fuel is fissioned in a fast breeder reactor (FBR) and again reprocessed. However, 


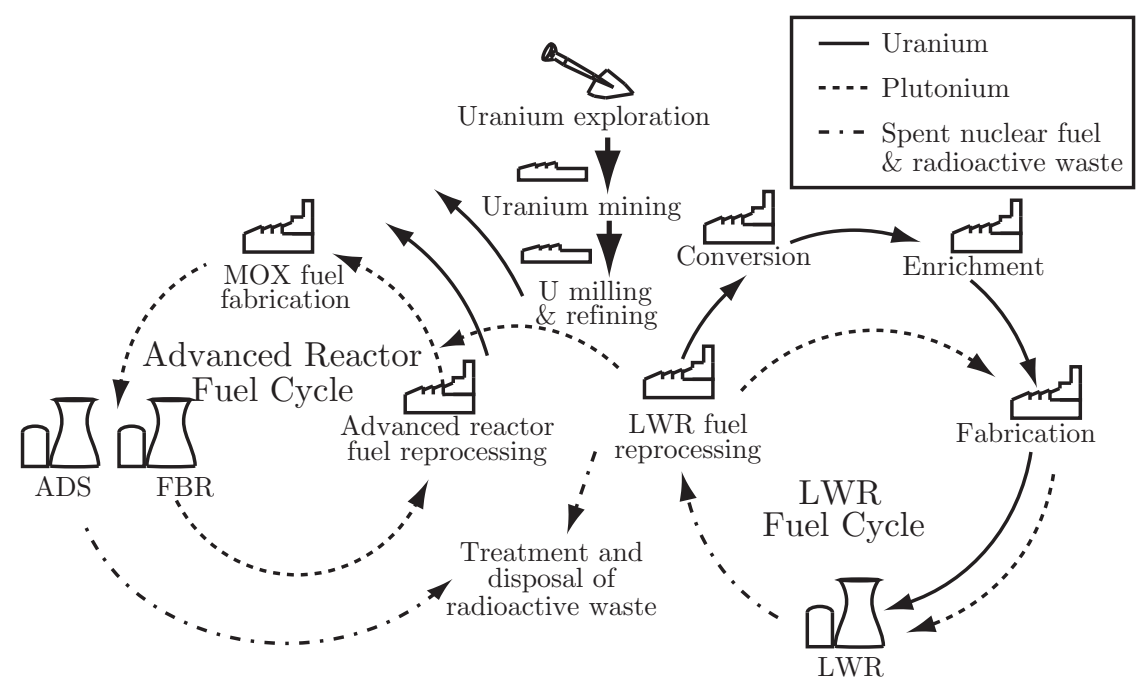

Figure 2.1. Nuclear fuel cycle.

the fuel could be burned in a once through subcritical system like an accelerator driven system (ADS) before being disposed, without additional reprocessing.

The work presented in this manuscript is applied in the MOX fuel fabrication and in the reprocessing steps of the advanced reactor fuel cycle.

Over the next points a detailed description from each of the steps in the nuclear fuel cycle is presented.

\subsubsection{Exploration of Uranium Deposits}

A uranium deposit is a mass of naturally occurring mineral from which uranium could be exploited. There are several types of uranium deposits, depending on the chemical composition of the rocks forming the terrain orography where the uranium deposit is placed. The deposits are collected in a classification in the OECD/NEA-IAEA Red Book. This deposit classification is established and updated by experts from six countries and from the IAEA. Nowadays a database call UDEPO is continuously updated and improved and is placed in a website for the public access [30].

The exploration of uranium deposits follows different phases in which 
the decision to continue or terminate the exploration is taken. Currently these phases are: area selection, reconnaissance, follow-up and detailed exploration [27]. Geologists make geochemical analyse in order to select the most suitable exploration methods. Furthermore an economic evaluation of the grade and characteristics of the orebody must be completed.

First deposits were close to the surface and easy to discover using Gamma radiation detection techniques. Gamma radiation indicates the activity of the uranium and the elements of the uranium decay chain. In the case of depleted uranium (nearly pure ${ }^{238} \mathrm{U}$ ), virtually all of the gamma activity will originate from ${ }^{238} \mathrm{U},{ }^{234} \mathrm{Th}$ and ${ }^{234} \mathrm{~Pa}$. Enriched uranium will have somewhat higher gamma activity proportional to the contribution of ${ }^{235} \mathrm{U}$ and ${ }^{231} \mathrm{Th}$.

With time the deposits of uranium are more difficult to find due to their location in a deep underground. Advanced technologies like satellite imaging or remote sensing techniques are used to explore these deposits.

\subsubsection{Mining and Milling}

There are three main alternatives to recover the uranium ore: the open pit mining method which is used for deposits close to the surface, the underground mining method which is used for the deep deposits and the in situ leaching (ISL) or in situ recovering (ISR) mining method to recover uranium in solution [31].

During the mining processes a prevention and minimization of the contamination and adverse impacts produced on the surface and subsurface environment and on the mine workers and local population should be considered.

Mining uranium from its ores generally follows the next steps [27]:

1. Size reduction: The ore is detonated to provide a suitable range of particle sizes required for effective leaching and to produce the slurry pumped through the processing circuits. The ore is crushed to separate the uranium metal from unwanted rock material.

2. Leaching: Uranium is dissolved from the ground ore using either acid or alkaline (carbonate) solutions with or without heating and with or without the addition of oxidants. The leaching separates chemically the solids from the condensed uranium solution. 
3. Solid-liquid separation and washing: The pregnant liquid is separated from the leached ore, which is then washed to remove any remaining dissolved uranium.

4. Purification and concentration: The uranium liquid is concentrated and purified using either ion exchange resins or solvent extraction, or both.

5. Precipitation and solid-liquid separation: An uranium oxide concentrate $\mathrm{U}_{3} \mathrm{O}_{8}$ (yellow cake) is precipitated and filtered to separate the water by dissipation and centrifugation.

6. Drying or calcining: The concentrate is dried or calcined and packed in steel drums lined with polyethylene.

7. Tailings disposal: Treatment of waste and recovering and recycling the water used.

After these operations, the packaged yellow cake is transported to the refineries.

\subsubsection{Refining and Conversion}

The yellow cake is refined to remove the impurities producing high-purity uranium trioxide $\left(\mathrm{UO}_{3}\right)$. Afterwards, according to the reactor where the $\mathrm{UO}_{3}$ will be used, it is converted either into powdered uranium dioxide $\left(\mathrm{UO}_{2}\right)$ used in the fabrication of pellets for the HWR, or uranium hexafluoride $\left(\mathrm{UF}_{6}\right)$, which is enriched and converted in enriched $\mathrm{UO}_{2}$ in order to fabricate LWR pellets. $\mathrm{UF}_{6}$ is the only uranium compound used to perform enrichment because its thermal stability and relatively high volatility.

\subsubsection{Enrichment}

The natural level of ${ }^{235} \mathrm{U}$ is $0.7 \%$. Most reactors require contents in an average level of $3-5 \%$. The enrichment is the procedure to increase the concentration of the ${ }^{235} \mathrm{U}$ in the $\mathrm{UF}_{6}$. During the process the lighter ${ }^{235} \mathrm{U}$ atoms are separated from the heavier and predominant ${ }^{238} \mathrm{U}$ atoms in order to concentrate them. This procedure can be done by two different methods: gaseous diffusion and centrifugation [28]. 
During the enrichment depleted uranium with a ${ }^{235} \mathrm{U}$ - content of approximately $0.2 \%$ is generated. This material may be classified as a byproduct or as a waste.

\subsubsection{Fuel Fabrication}

The fabrication is the last operation before loading the uranium fuel in the reactor and using it. There are liquid and solid forms of fuel. The assembly of these fuels, packed in fuel rods, depends on the reactor where they are burned. A description of the fuels related with this manuscript is presented in 2.3 .

\subsubsection{Electricity Generation}

Most of the electricity provided in buildings is generated in electric power generating plants. The reactor in a nuclear power plant works similar to the boiler in a fossil fuel plant. As seen in Figure 2.2, the nuclear fuel supplies heat to boil water and produces steam, the pressure of which rotates turbines. The movement of the turbines turns the shaft of the generator, inside which electricity is produced by the interaction of magnetic fields generated with coils of wire.

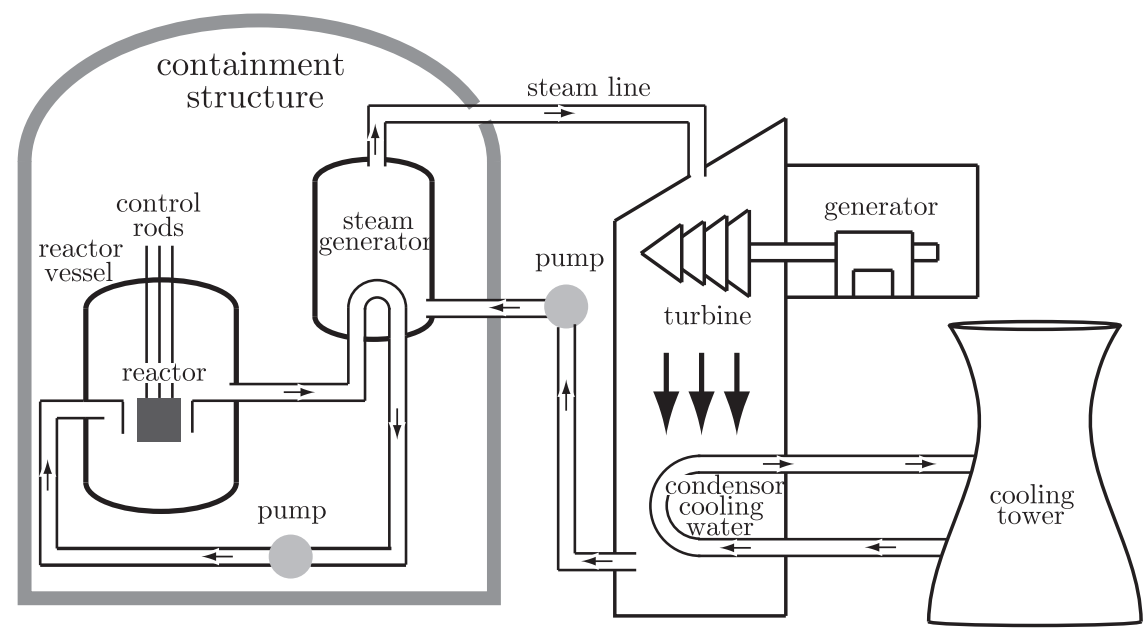

Figure 2.2. Nuclear fuel electric power generating plant 
The thermal reactor is constituted of five basic parts, which are: the core, a moderator, the control rods, a coolant and shields, whereas the fast reactor does not feature a moderator. The core contains the nuclear fuel. The moderator slows down the neutrons produced by fission of the fuel while the control rods, filled with pellets of substances like hafnium or cadmium and inserted into the core of the reactor, control the chain reaction absorbing neutrons and slowing down the reaction.

As the amount of heat generated by fission is huge, a coolant carries this heat away from the reactor to the steam generator. Shields are used to prevent the environment from radiation.

As seen before, there are different types of reactors: boiling water reactors (BWR), pressurized water reactor (PWR), pressurized heavy water reactor (PHWR), fast breeder reactor (FBR), supercritical water reactor (SCWR) or high temperature gas cooled reactors (HTGR). The most common reactors are described below.

\section{Light Water Reactor}

Light water reactors (LWR) are thermal reactors where light water (i.e. $\mathrm{H}_{2} \mathrm{O}$ ) is used as a moderator and coolant, i.e. PWRs, BWRs and water cooled, water moderated power reactors (WWERs). Worldwide the LWRs are the dominant type of nuclear plants [29]. Moreover, advanced LWR designs building are being developed with different power ranges in several countries for future energy needs.

LWR produces heat by controlled nuclear fission in its core. The core consists of nuclear fuel and control elements. The LWR fuel is mostly enriched $\mathrm{UO}_{2}$ in form of pellets that fills the rods forming the fuel assembly.

In BWRs, the electricity is generated in power turbines directly fed by the steam coming from the primary cooling circuit. This is not the case of the PWR, where a heat exchanger connects two circuits to maintain water under pressure. The reactor is placed in the primary circuit and the steam is generated in the secondary circuit with the heat transmitted through the exchanger. A condenser turns back the steam into water after flowing through the turbines. 


\section{Fast Breeder Reactor}

Fast Breeder Reactors (FBR) are primarily Liquid metal cooled fast reactors (LMFRs), that have been widely developed [29]. FBR are capable to produce more fuel than it consumes during the fission process turning fertile elements into fissile ones by neutron capture. Moreover, they offer the possibility to burn actinides with the intention of reducing waste, and therefore they are one of the most promising technologies of the Generation IV reactors.

As a comparison with the thermal reactors, FBR produce sixty-toseventy times more energy from plutonium breeding than the LWR from uranium do. Because of the different spectrum fast reactors can reduce the plutonium stockpiles generated from the fission of uranium in LWR. Present fast reactor concepts are the Gas-cooled fast reactor (GFR), the Sodium-cooled fast reactor (SFR) and the Lead-cooled fast reactor (LFR). They could be used in a closed nuclear fuel cycle and contribute to the reduction of the disposal time of the high level radioactive waste.

\section{Accelerator Driven System}

Both the LWR and the FBR are critical reactors, i.e. once the fission of the fuel starts, a chain reaction is sustained. An Accelerator-driven system (ADS) is a subcritical reactor that uses additional neutrons from an outside source and produces fission without criticality. The neutron source can be a particle accelerator producing neutrons by spallation [29].

The potential advantages of ADS systems are low waste production, high transmutation capability, enhanced safety characteristics and better long term utilization of resources (e.g., with thorium fuels). Despite being intended to be used for the burning of spent nuclear fuel coming from the LWR before to be disposed, the ADS remains a reactor and could be used in a closed fuel cycle providing electricity while burning waste [32].

\subsubsection{Reprocessing}

The nuclear fuel has a limited life. As the fuel in other systems, it should be replaced by new fuel after its use. The spent fuel contains residual ${ }^{235} \mathrm{U}$, plutonium $(\mathrm{Pu})$ that is generated from the absorption of neutrons of the ${ }^{238} \mathrm{U}$, minor actinides, and other fission products and wastes that should be reprocessed (see annex for more information about the transuranic ele- 


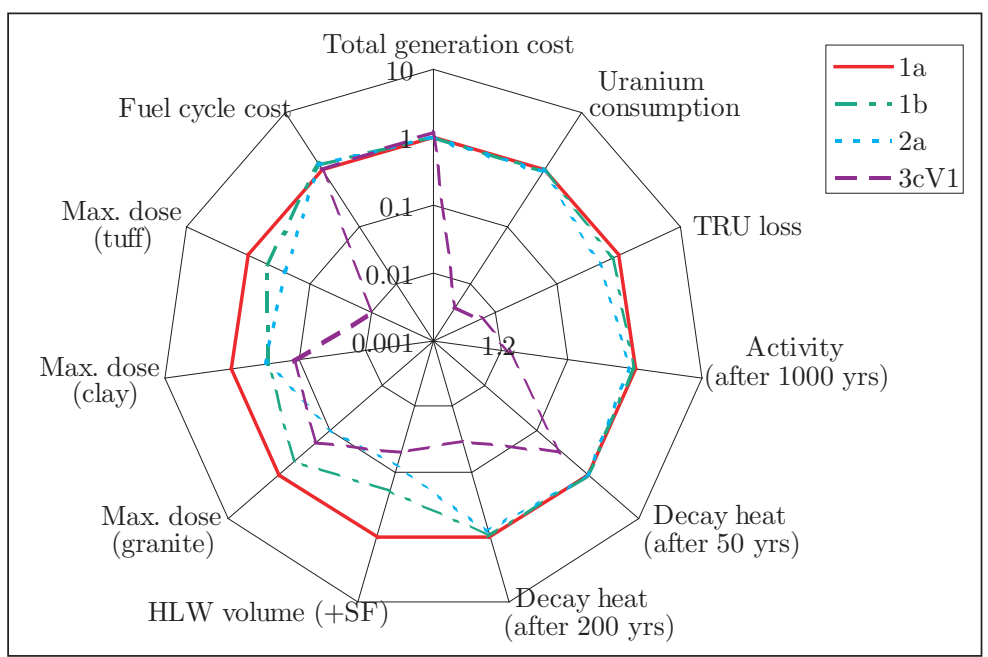

Figure 2.3. Comparison of 11 representative indicators for various fuel cycle schemes. 1a) Once-through PWR (reference); 1b) $100 \%$ PWR, spent fuel reprocessed and Pu reused once; 2a) $100 \%$ PWR, spent fuel reprocessed and multiple reuse of $\mathrm{Pu}$; 3cV1) $100 \%$ fast reactors and fully closed fuel cycle. Source: OECD/NEA, 2006

ments). There is a need for nuclear waste management [3] and most of the elements of the spent fuel can be reused, e.g. the $\mathrm{Pu}$ can be used to produce mixed oxide (MOX) fuel and burned in a reactor.

Figure 2.3 (source [25]) shows the impact of several advanced nuclear fuel cycle scenarios and how they behave in consideration of aspects like: uranium consumption, transuranic waste loss, activity and volume of the high level waste (HLW), maximum dose released to the biosphere or costs. The decay heat is one of the main inputs to be considered for the design of the underground repositories. The maximum allowable density of disposal in granite, clay and tuff formations is determined by thermal limitations.

The fully closed fuel cycle shows a scenario where almost all the represented indicators are considerably reduced without supposing an extra cost. Consequently this thesis finds one of the main motivations to participate in the reprocessing stage of the nuclear fuel cycle, perhaps contributing to the reduction of these indicators. 


\subsubsection{Geological Disposal and Radiotoxicity}

The nuclear waste is classified in three categories depending on the radioactivity as follows:

- Low-level waste (LLW): Waste with small amounts of radioactivity that can be disposed near to the surface until the radioactivity decays.

- Intermediate-level waste (ILW): Waste that comes mostly from nuclear industry. It requires containment and isolation and a disposal of the order of tens to a few hundred metres. It needs no or limited provision for heat dissipation.

- High-level waste (HLW): generally describes spent fuel from the reactors that generates significant quantities of heat by radioactive decay. The disposal should be deep and in stable geological formations at usually several hundreds of metres bellow the surface.

Currently research and development of disposal systems is carried out. The Grimsel Test Site (GTS) was established in 1984 supporting a wide range of research projects on the geological disposal of radioactive waste, with partners from Europe, Asia and North America as a Unique facility. GTS provides realistic conditions analogous to that of a repository site. Figure 2.4 shows a storage model (researched by NAGRA [33]) of a fuel assembly disposed underground, where the safety barriers of the HLW are easy differentiable: the waste matrix containing radioactive material is placed in the center; it is then surrounded by a metal container that meanwhile is surrounded by a buffer and placed inside the host rock, which provides the geological barrier.

Partitioning and Transmutation (P\&T) of the actinide elements appears as a new concept in a closed fuel cycle, where unlike in the open cycle directly associated with LWR, the spent fuel is reprocessed before it is disposed. P\&T is considered as a solution to slow down the load of geological disposal and therefore has a potential impact on the waste disposal issues compared to the direct disposal of spent fuel.

Furthermore, P\&T allows designing the radiotoxicity (i.e. toxicity of radioactive elements) which is a measurement of the potential hazard to humans from ingestion, inhalation and exposure to the contained radionuclides. The International Commission on Radiological Protection (ICRP) 


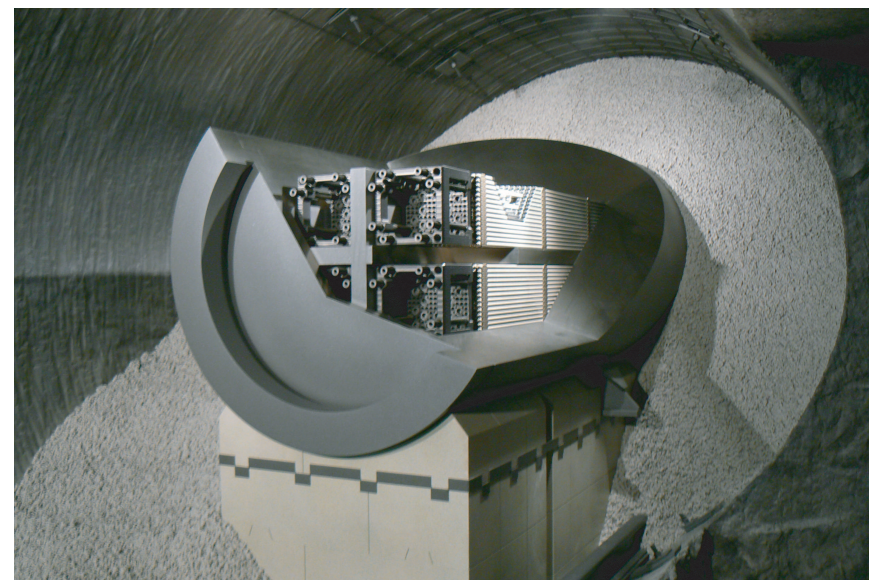

Figure 2.4. Storage model for nuclear spent fuel in the Grimsel Test Site in the Swiss Alps. Picture taken by the author of this manuscript during a NAGRA guided visit in the GTS.

publishes continuously information that relates the radiation emanating from any given isotope to a health hazard [34]. The radiotoxicity is measured with dose conversion factors that are continuously being re-evaluated and updated. These dose conversion factors depend on the type of radiation. Figure 2.5 (source [2]) provides an example of the ingestion radiotoxicity for the different elements of the spent fuel compared with the radiotoxicity of the natural uranium ore.

From this figure it can be concluded that the fission products are discharged in a shorter period (1000 years) than the minor-actinides (MA) and plutonium (10000 years) and become less radiotoxic than the natural uranium ore. Consequently a closed fuel cycle scenario for the treatment of these elements before disposal in a geological repository is preferred.

\subsection{Solid Nuclear Fuel}

The main intention of the project described in this thesis is to find a solution for the production of reprocessed nuclear fuel from the existing spent fuel. The most spread nuclear fuel is in the physical form of pellets. Nevertheless, because of the spent fuel radioactivity, the pellets are not the optimal 


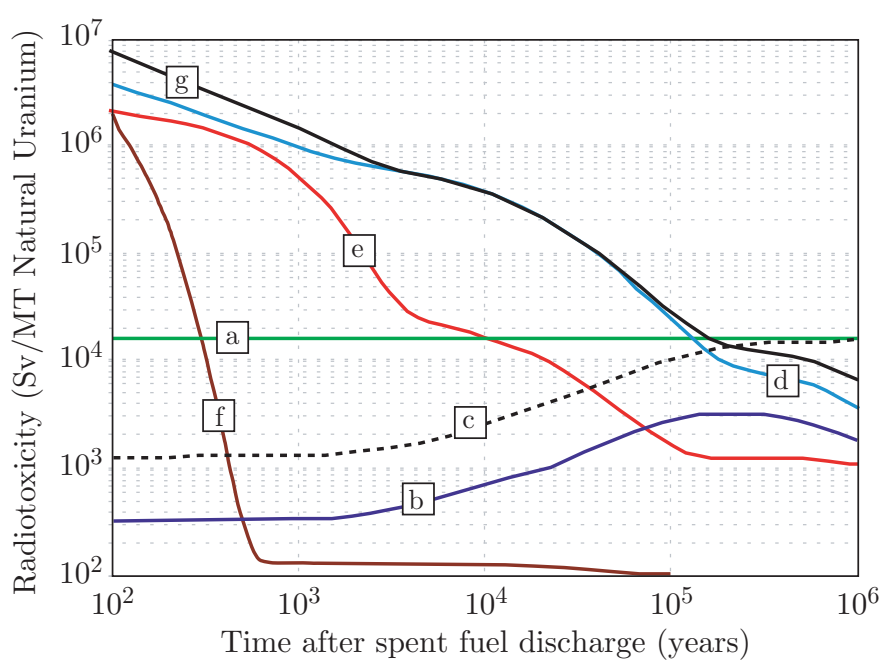

Figure 2.5. Ingestion radiotoxicity for spent fuel. a) Uranium ore; b) Uranium and decay products; c) Radiotoxicity of natural uranium and decay products; d) Plutonium and decay products; e) Minor actinides and decay products; f) Fission Products; g) Total. Source: Salvatores, 2011

solution to handle the components. Therefore, particle fuel provides a new concept to deal with radioactivity difficulties.

\subsubsection{Pellets}

After the conversion of $\mathrm{UF}_{6}$ and/or $\mathrm{UO}_{3}$ in enriched uranium dioxide $\mathrm{UO}_{2}$ the manufacture of pellets starts. The $\mathrm{UO}_{2}$ powder enters in a homogenization process to ensure uniformity in its particle size and apply additives to ensure a satisfactory microstructure and density in the pellets.

The powder is fed into dies and pressed at high speed into cylindrical pellet form. Afterwards, they are sintered at temperatures up to $1750{ }^{\circ} \mathrm{C}$ in a furnace and controlled under low pressure to consolidate them. Then the pellets are modelled with grinding machines to have exact dimensions (usually about one $\mathrm{cm}$ diameter and one $\mathrm{cm}$ long) and finally they pass a rigorous dimension and integrity quality control [35].

Some of the pellets may contain burnable absorbers (such as Gadoli- 
nium and Boron), in order to compensate the reduction of the neutron flux during irradiation, namely to limit the early reactivity in the fuel.

The pellets are filled in rods, also known as cladding, which are made usually of zirconium alloy because of its stability (mechanic and chemical) and its low cross section to let the neutrons through, or steel in case of fast reactors. The rods are flushed with helium and pressurized, certifying a free space to prevent the thermal expansion of the pellets and the formation of fission product gases. A spring is placed in this free space to avoid the movement of the pellet.

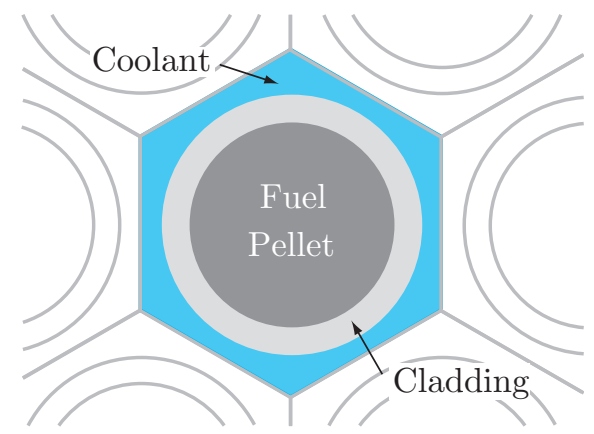

Figure 2.6. Schema of a fast reactor fuel assembly of pellets.

Cladding is fixed in assemblies, which structures are designed with tight tolerances, being resistant to chemical corrosion, high temperatures, vibrations and fluid or mechanical impacts. The structures are made from zirconium alloy and steel and permit inter-spaces between rods allowing the coolant to pass trough. Both rods and parts of the structure must be as transparent as possible to neutrons. A schematic overview of a fast reactor fuel assembly is shown in Figure 2.6

\section{MOX fuel}

Mixed Oxide (MOX) fuel consists of plutonium and natural or depleted uranium. MOX fuel is fabricated in reprocessing plants and follows the same procedure with pressing and grinding machines as the $\mathrm{UO}_{2}$ pellets. However, the fabrication process is carried out in glove-boxes with controlled atmosphere where Argon gas is used to maintain chemical inertia [36] (carbide is chemically active for oxygen and water). 


\subsubsection{Particle Fuel}

As an alternative to the fuel in form of pellets, the particle fuel has been extensively researched. Particle fuel appears to simplify the fuel fabrication process and offers high relevance advantages. There are two major types of particle fuel, Vibropack Fuel also known as Vipac fuel and Sphere-Pac Fuel which will be the one fabricated following the description of this manuscript. Figure 2.7 shows a comparison of both fuel structures.

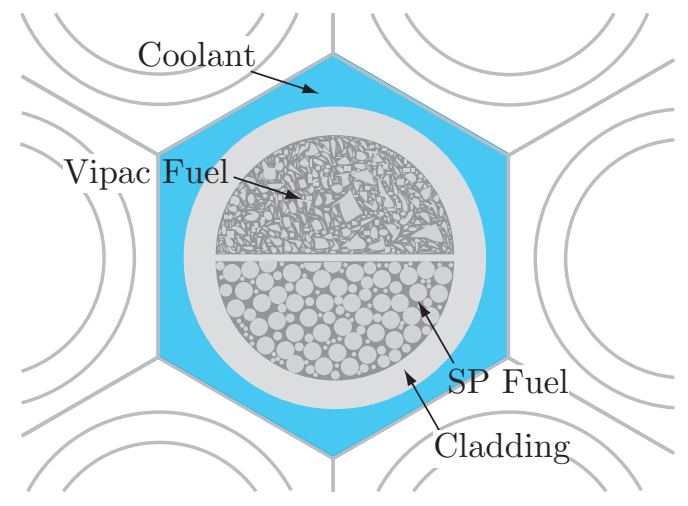

Figure 2.7. Comparative schema of a fuel assembly of Vipac (up) and Sphere-pac (down) fuel.

The use of particle fuel instead of pellets implies changes in several important properties. The structure integrity and the mechanical properties are modified because of the higher temperature increase. There is more fission gas release and a lower conductivity due to a larger surface. The introduction of fissile material is an option to adjust the thermal behaviour and the porosity. In any case safety aspects should be researched considering cladding failure scenarios.

\section{Vipac (Vibropack) Fuel}

The concept of Vipac Fuel appears in the late 1950s as a possibility to avoid the MOX pellets fuel manufacturing [37, 38]. This nuclear fuel consists in filling cladding with randomly shaped particles by "Vibration-Packing". The fuel particles are scraped off from electrodes used in a dry reprocessing (oxide electrowinning). They are sieved into several sizes (between 3 and 7 classes) which are filled simultaneously and in the right ratio into the pin, in 
order to reach high densities. Additionally an oxygen absorber is introduced with the fuel to minimize the chemical reactions inside the cladding. Vipac Fuel is also considered for the fabrication of MA-containing MOX fuel, for its use in fast reactors.

\section{Sphere-Pac Fuel}

As in the Vipac fuel, Sphere-Pac (SP) fuel consists also in particles filled directly in the cladding. The main difference with the Vipac fuel, as seen in Figure 2.7, is the sphericity of the particles in the SP fuel, which enables to predetermine their arrangement inside the cladding. These spherical particles are also of several sizes to aim for a maximal fuel density. Another improvement is related with the production process [39]. The particles are formed by means of an aqueous method that assures an excellent distribution of the fissile material in the matrix forming a solid solution in the ceramic and allowing a good control of the particle fraction dimensions. Moreover, advantages like powder reduction and less use of mechanical devices, are also related to this method.

\subsection{Sphere-Pac Fuel Production}

Several methods are considered for the production of spherical particles from solutions and suspensions, where powderless handling and chemical homogeneity of the solutions are the main advantages. Sol-gel methods are the more widespread due to their resistance to radioactive environments. There are three common methods (see Figure 2.8): internal gelation where the gelation is triggered applying heat, external gelation which is driven by a mass transfer of ammonia and water extraction process, where the water is extracted into organic alcohols.

Gelation is a chemical process through where isolated building blocks are destabilized and react together to generate a three-dimensional network. The destabilization is initiated by addition of an external catalytic agent, generally either an acid or a base. The following points offer a short description of the methods. 


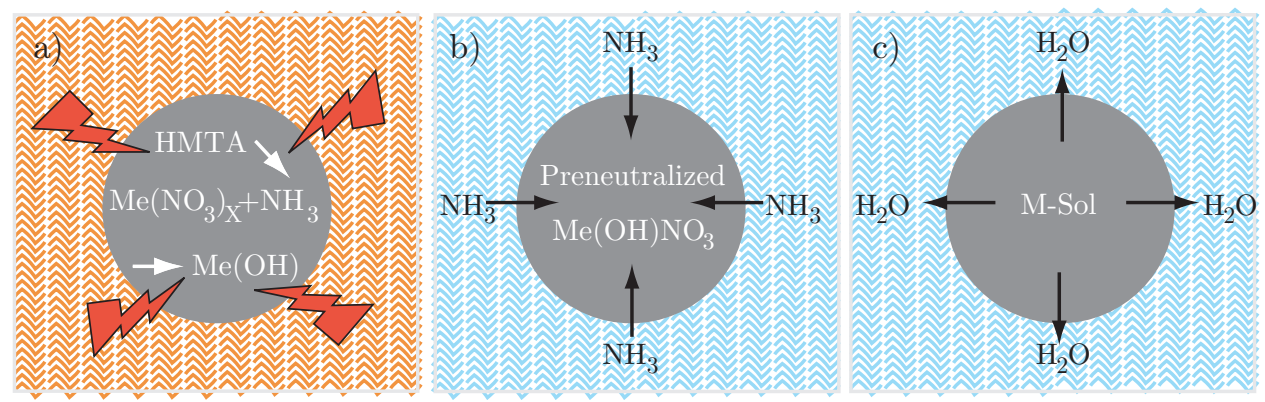

Figure 2.8. Schema of the sol-gel processes: a) internal gelation, b) external gelation, c) water extraction process.

\subsubsection{Water Extraction Process}

Water extraction process, represented in Figure $2.8 \mathrm{c}$ ), consists of a gel microspheres formation while an organic liquid with water solubility, which contains a surface active agent, is precipitated into a hydrous oxide solution. This procedure is also historically called ORNL process [40]. The water is extracted to cause the gelation and afterwards a drying, calcination and sintering processes of the gelated spheres is done. The organic liquid is recovered for reuse.

\subsubsection{External Gelation}

External Gelation is the largest-scale process used in the production of particle fuel. Germany reports a long term experience in the high quality production of $\mathrm{UO}_{2}$ particles [41]. A schema of the external gelation is represented in Figure $2.8 \mathrm{~b}$ ).

A uranium feed solution is precipitated from a nozzle in form of drops, which pass through an ammonia gas layer hardening its surface to form spheres. Afterwards the spheres are collected in an aqueous concentrated ammonium hydroxide bath, where they are gelated due to the transfer of ammonia and aged. Then a washing procedure is applied followed by a drying, calcination and sintering processes. 


\subsubsection{Internal Gelation}

The particularity of internal gelation (schema in Figure 2.8 a)) is that all components reagents are mixed together in a homogeneous solution at first. The base (ammonia) is generated in-situ by thermal decomposition of a precursor, hexamethylenetetramine (HMTA). With the internal gelation a wide range of controlled sizes of spheres can be manufactured and it is possible to use the procedure for non-oxide ceramics, e.g. nitrides and carbides.

A temperature increase to trigger the gelation was the reason of researchers to look for the best heating transfer medium, going from silicon oil to the microwaves $[8,9]$. At the Paul Scherrer Institut (PSI) in Switzerland the internal gelation has been extensively investigated [10, 12-14, 42]. With this thesis, also developed at PSI, a better understanding of the molecules interaction with the microwaves during heating in the internal gelation procedure is researched.

Over the following sections a detailed state of the art of the internal gelation procedure is presented.

\section{Chemistry of the internal gelation}

For reasons of versatility in the early stage of the project development where the thesis is involved, the feed solution utilized in the internal gelation process consists of an inactive metal surrogate. Cerium is chosen because of its physico-chemical similarity with actinide elements, especially Plutonium $(\mathrm{Pu})$ [43], [44]. The sample is a mixture of metallic solution (ceric ammonium nitrate) and the hexamethylenetetramine (HMTA) solution (composed of HMTA, urea and water).

$$
\left(\mathrm{CH}_{2}\right)_{6} \mathrm{~N}_{4}^{+}+10 \mathrm{H}_{2} \mathrm{O} \longrightarrow 4 \mathrm{NH}_{4} \mathrm{OH}+6 \mathrm{CH}_{2} \mathrm{O}
$$

where:

$$
\mathrm{NH}_{4} \mathrm{OH}=\mathrm{NH}_{4}^{+}+\mathrm{OH}^{-}
$$

The HMTA $\left(\left(\mathrm{CH}_{2}\right)_{6} \mathrm{~N}_{4}\right)$, as seen in the reaction 2.1 , decomposes with temperature increase and yields ammonium hydroxide 2.2 and formaldehyde $\left(\mathrm{CH}_{2} \mathrm{O}\right)$, which causes the gelation within the nitrate solution. The hydrolysis of HMTA releases $\mathrm{OH}^{-}$ions that react with the cerium ions to precipitate as cerium hydroxide, turning into cerium oxide (or carbide if carbon-black is added to the fuel solution) after a heat treatment. 
The reaction occurs also at a room temperature taking more time. In order to prevent a premature reaction, both solutions are cooled down to a temperature between $0{ }^{\circ} \mathrm{C}$ and $5{ }^{\circ} \mathrm{C}$ and mixed shortly before the production.

\subsection{State of the Art of the Internal Gelation SP Fuel Production}

The Sphere-Pac fuel production by internal gelation needs a heat source to initiate the solidification of the mixed solution, i.e. the gelation. The widespread heating source has been a silicon oil bath $[8,9]$, but the solution, which is going to be gelated, has already all the necessary gelation ingredients. Therefore there is no need of a bath which implies a post-procedure to clean both the silicon oil and the gelated spheres.

During the eighties the idea of using microwave as a heating source was introduced $[12-14,42]$ minimizing the steps during the particles production by internal gelation. Figure 1.1 presents a comparison of the silicon oil and the microwave based manufacturing. In subsequent years several microwaves systems working in different frequency bands were developed [1518]. Currently a new program called Platform for Innovative Nuclear Fuels (PINE) (description in [7]) rescues the idea of the microwave internal gelation with the main intention of transfer the concept to a large scale industrial production.

A detailed description of both variants and their advances are presented below. In the following chapters this manuscript presents the advances which contribute to the PINE project and the microwave internal gelation improvement.

\subsubsection{Silicon Oil Internal Gelation}

A status report from the Netherlands in the KEMA laboratories at Arnhem [45] describes the three sol-gel processes above mentioned. In the case of internal gelation it is reported that particles up to a size of $700 \mu \mathrm{m}$ are gelated with temperatures between $50{ }^{\circ} \mathrm{C}$ and $60{ }^{\circ} \mathrm{C}$ depending on the water content of the medium which is Alphanol-79. However, in case of particles in the millimetre range they notice that two factors should be 
considered: a linear shrinkage of the big particles and the preservation of the shape of the drop during solidification.

To achieve these goals Hermans et al. [45] considered two main aspects in the gelation system. Firstly they chose an organic medium with a high density and high interfacial tension towards water, e.g. tetrachloroethylene. In a second level they rise up the speed of the gelation process by increasing both the temperature of the organic medium and the HMTA concentration in the feed solution.

These early studies introduced multiple scientific research about internal gelation, which was the subject of some past work also at PSI [46].

Figure 2.9 shows a schema of the production unit used at PSI [39]. From left to right the schema describes the steps followed during the internal gelation process. Firstly, the feed solution is prepared mixing the nitrates, the urea and the HTMA and is kept at roughly $270 \mathrm{~K}-272 \mathrm{~K}$ to prevent an early gelation.

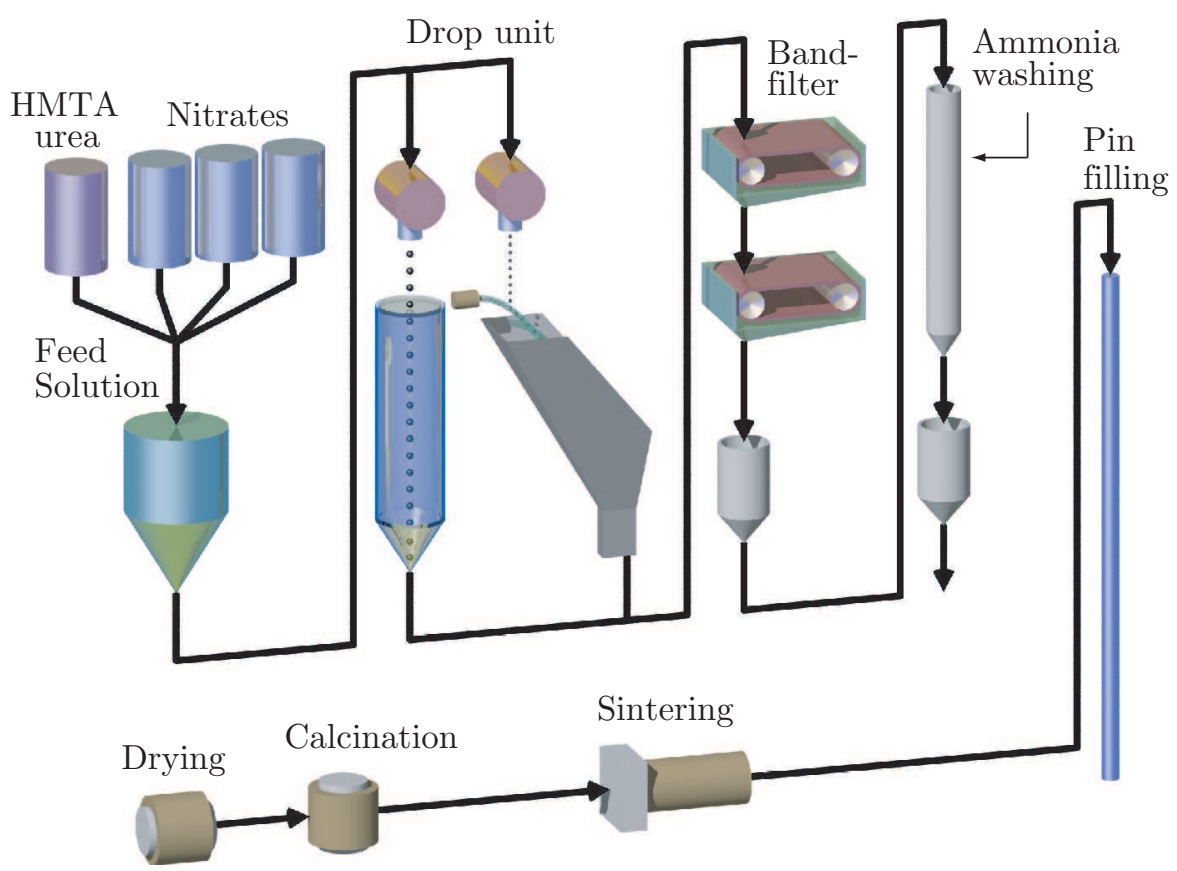

Figure 2.9. Schema of internal gelation production unit used in the past at Paul Scherrer Institut. 
The drop unit produces different sizes of particles (from $40 \mu \mathrm{m}$ to 1.5 $\mathrm{mm}$ typically) by changing the nozzle and the induced vibration. The schema presents two different drop collectors: on one side the small spheres (15000 per second) are produced by vibration and they impact into an oil jet which flows into an oil bath; on the other side the large spheres (6 per second) dropped by gravity directly into the oil bath. The oil jet in the case of the small drops prevents agglomeration before the solidification. In both cases the hot silicon oil is at roughly $373 \mathrm{~K}-379 \mathrm{~K}$ to trigger the gelation procedure.

The gelated particles are transported through a filter to separate the oil by a small vacuum unit. The oil is filtered removing the water picked up during the process and then it is recirculated into the oil circuit. The spheres are washed with an organic solvent and again filtered to removed the potentially oil contaminated organic solvent, which is also recycled.

The collected spheres are redirected to a final washing in ammonia solution which removes the residual reaction products and the excess of nitrates. Also the ammonia is recycled. The following step in the process consists on a thermal treatment including: drying in hot air, calcination and sintering of the spheres[10, 47]. Finally the spheres are filled directly into the pin forming the so-called Sphere-Pac fuel.

\subsubsection{Microwaves Internal Gelation}

Although the internal gelation production avoids the powder handling, the unit described before requires more space than the production unit in the case of pellets fabrication and it presents some disadvantages as the silicon oil and ammonia recycling. These reasons caused the search for an optimization of the process resulting in the proposal of the microwaves as a potential advanced approach.

As the feed solution contains all the ingredients to conduct the solidification, the microwaves provide the heat needed to run the gelation and (as seen in Figure 1.1) reduce the steps during the production of the spheres.

During the eighties several researchers proposed microwave heating for the production of SP-fuel by internal gelation $[12-15,17,18]$. The relevant studies with the justification of each author is here presented in two sections: the work developed in X-band and the work developed in S-band. 


\section{X-band}

Knotik et al. [12] used the $14 \mathrm{GHz}-14.5 \mathrm{GHz}$ frequency range. They tested several X-band microwave cavities with square cross-sections between $10 \times$ $10 \mathrm{~mm}^{2}$ and $14 \times 14 \mathrm{~mm}^{2}$ and lengths between $150 \mathrm{~mm}$ and $1000 \mathrm{~mm}$. Moreover all the designs sustained at least one resonant frequency with a $\mathrm{TE}_{10 p}$ mode $(p=15 \cdots 100)$ in the proposed frequency range. The optimum operational cavity was $0.3 \mathrm{~m}$ long. The drops continued in a $5 \mathrm{~m}$ free fall after being heated in the cavity to ensure their solidification without any further treatment. That made the complete system around $7 \mathrm{~m}$ height.

The amplifier provided an incident power until $250 \mathrm{~W}$, being necessary only $80 \mathrm{~W}-90 \mathrm{~W}$ to increase the temperature by $85 \mathrm{~K}$ and get satisfactorily gelated spheres. Lower power levels produced deformation in the particles when impacting in the collector.

Ledergerber $[13,14]$ was developing meanwhile also a X-band system (Figure 2.10) in $8 \mathrm{GHz}-14 \mathrm{GHz}$ frequency range. The cross-section of the resonator matched with WR90 waveguide dimensions. The cavity was working in the $\mathrm{TE}_{10(11)}$ mode. The spheres fell through the cavity from top to bottom provided openings during $0.12 \mathrm{~s}$. A quartz tube was placed inside to protect the cavity from contamination. As seen in Figure 2.10 (source [14]), the Ledergerber system is shorter than the Knotik system. The drops are deposited in the collector after they pass through the cavity.

Incident powers of $80 \mathrm{~W}-100 \mathrm{~W}$ resulted in temperature gradients of $60 \mathrm{~K}-80 \mathrm{~K}$ within the drops, giving gelated spheres with the same quality as the ones generated by silicon oil internal gelation.

\section{S-band}

Yamagishi et al. $[15,17]$ wanted to use an inexpensive commercial microwave power source operating at the engineering frequency $2.45 \mathrm{GHz}$ instead of the communications reserved frequency band (X-band) used by Ledergerber. The cavity was designed for a $\mathrm{TE}_{10}$ mode with a single vertical E-field maximum. Therefore, the drop travelled always through a maximal intensity during its residence time inside the cavity. The length of the cavity was shorter than the one in Ledergerber design justifying the avoidance of spheres deformation during gelation.

As the working frequency of the cavity varies with the temperature, the placement of the quartz tube and the presence of droplets, it was proposed 


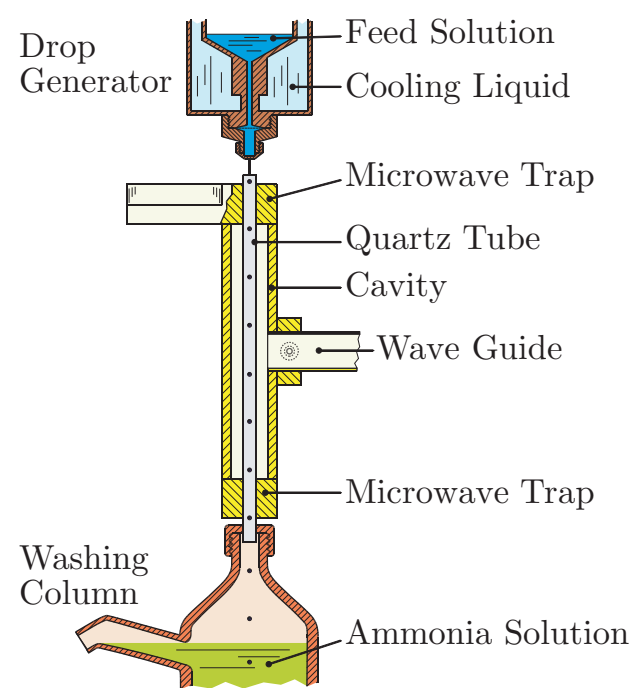

Figure 2.10. Design of the microwave applicator by Ledergerber at Paul Scherrer Institut.

to have the cavity monitored with a spectrum analyser in that case. A frequency range was defined by a minimum and maximum value, $\mathrm{f}_{\min }$ and $\mathrm{f}_{\max }$ respectively, and the quartz tube was moved up and down with a drive mechanism to tune the cavity working frequency in that range.

Incident power between $4 \mathrm{~kW}-5 \mathrm{~kW}$ was used over several experiments, showing a temperature rise in all cases of $80 \mathrm{~K}-85 \mathrm{~K}$ and satisfactory gelated spheres.

Rosin et al. [18] tried also to increase the heating rate of the droplets compared to the X-band units. They proposed three main points to aim for this heating rate increase in their studies: a) an increase of the power level, b) a reorientation of the droplet movement in the direction of the maximum electric field, and c) an increase of the residence time in the microwave cavity. They selected the b) and c) options for the design of a microwave unit at $2.45 \mathrm{GHz}$, while a) was implied by the designed cavity $(0.5 \mathrm{~kW}-2 \mathrm{~kW})$, but reducing the level with respect Yamagishi case.

As a novelty, the drops were coming out from a nozzle placed inside the cavity on the top part and travelling parallel to the electric field orientation through the cavity. This design wanted to extend the residence heating time 
of the drop formation. The dimensions of the cavity, which works with a $\mathrm{TE}_{10}$ mode, matched with a WR340 waveguide and includes a shorting plunger to adjust the E-field distribution.

The reported work shows that they had several limitations and drawbacks, the drops where exploding and the feed solution had a premature gelation because of the introduction of the nozzle inside the cavity.

Table 2.1 shows a comparison of the main parameters of the designs presented in the previous literature. As a conclusion it is obvious to see that the heating efficiency falls off with the microwave frequency decrease.

\begin{tabular}{lcccc}
\cline { 2 - 5 } & $\begin{array}{c}\text { Knotik } \\
\text { et al. [12] }\end{array}$ & $\begin{array}{c}\text { Ledergerber } \\
{[13,14]}\end{array}$ & $\begin{array}{c}\text { Yamagishi } \\
\text { et al. [15, 17] }\end{array}$ & $\begin{array}{c}\text { Rosin } \\
\text { et al. [18] }\end{array}$ \\
\hline Power (W) & $80-90$ & $80-100$ & 5000 & $500-2000$ \\
Frequency $(\mathrm{GHz})$ & $14-14.5$ & $8-14$ & 2.45 & 2.45 \\
Dimensions & WR90 & WR90 & - & WR340 \\
Temp. gradients (K) & 85 & $60-80$ & $80-85$ & 40 \\
Gelated Spheres & Yes & Yes & Yes & No \\
\hline
\end{tabular}

Table 2.1. MIG literature comparative resume.

This thesis aims at the optimization of the work developed in X-band by Ledergerber $[13,14]$, providing a detailed analysis of the interaction between the microwaves and the feed solution in form of drops, and justifying the design of the cavity for the production.

\subsection{Conclusions}

In this chapter, the problem of MIG for the production of particle fuel was introduced. An overview of nuclear fuel manufacture was also presented. In a first level the chapter came up with the nuclear fuel cycle going through the steps which are part of the nuclear fuel, i.e. before, during and after its use. Secondly the widespread and well known pellets were presented together with newer concepts of solid nuclear fuel, e.g. particle fuel.

Next, the production methods for particle fuel were presented together with the motivation for the internal gelation. The chemistry of this SP production method was cited and briefly introduced. 
The last part of the chapter introduced the internal gelation research, which was done before to pick up the thread of this topic with the development of this manuscript in the context of the PINE project. The system model associated to a silicon oil as a heat carrier to trigger the gelation was shown, presenting the disadvantages that motivate the search of new manufacturing methods. Finally, the microwave techniques proposed by several authors were described for a comprehensive analysis of the subject of the thesis. 


\section{Thermal Model}

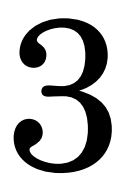

3.1 Introduction . . . . . . . . . . . . . . . . . . 39

3.2 The Heat Equation . . . . . . . . . . . . . . . . . 42

3.3 Heat Equation Solution by Numerical Methods . . . . 46

3.4 Boundary Conditions . . . . . . . . . . . . . . 50

3.5 Implementation and Results . . . . . . . . . . . . 53

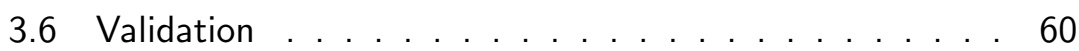

3.7 Conclusions . . . . . . . . . . . . . . . . . 67 



\section{Thermal Model}

The THERMAL BeHAVIOUR of a material heated by microwaves becomes the main research if the essential parameter to carry out the primary process of this dissertation is the temperature. This chapter presents a FDTD thermal model capable to inform each instant about the thermal behaviour of any point inside the heated material. This model includes the boundary conditions of the materials to approach the real experimental cases. In addition three different techniques are presented and utilized in order to validate this thermal model.

\subsection{Introduction}

Two regions in contact with different temperatures are subject to an energy transfer between them known as heat transfer, which flows always from higher temperature regions to lower temperature regions. The mechanisms of heat transfer are known as conduction, radiation and convection [48].

\section{Conduction}

Within a body the heat transfer from higher to lower temperature zones in a molecular level is the conduction. It also takes place between two different 
bodies in physical contact, from the body at a higher temperature to the body at a lower temperature. The more energetic molecules impact with the lower energy level molecules and exchange energy and momentum. In case of liquids and gases the continuous energy transport is similar, while in case of solids non electric conductors (dielectrics) heat is conducted by lattice waves caused by atomic motion.

The heat transfer rate per unit area normal to the direction of heat flow (a.k.a. heat flux) $Q_{f l u x}$ is proportional to the temperature gradient:

$$
Q_{\text {flux }}=-k_{T} \frac{\mathrm{d} T}{\mathrm{~d} x}
$$

where the constant $k_{T}\left(\mathrm{~W} \cdot \mathrm{m}^{-1} \cdot \mathrm{K}^{-1}\right)$ is the thermal conductivity, which is characteristic of the material. The minus sign is because the heat is transferred toward lower temperature regions. Fourier's law of heat conduction, which is represented in Equation 3.1 can be written in a general statement with the heat conduction rate equation as

$$
\mathbf{q}_{\text {flux }}=-k_{T} \nabla T
$$

where $\nabla$ is the three-dimensional nabla operator and $T$ is the scalar temperature field.

Considering a one-dimensional heat flow over a thin plane wall of $\Delta x$ thickness and $S$ surface, the heat transfer rate is given by:

$$
Q=\frac{k_{T} S}{\Delta x}\left(T_{2}-T_{1}\right)
$$

where $T_{1}$ and $T_{2}$ are the wall-face temperatures.

In conduction problems, the thermal diffusivity $\alpha$ is the relation of the thermal conductivity with the density $\rho\left(\mathrm{kg} \cdot \mathrm{m}^{-3}\right)$ and the specific heat capacity $c_{p}\left(\mathrm{~J} \cdot \mathrm{kg}^{-1} \cdot \mathrm{K}^{-1}\right)$ of the material.

$$
\alpha=\frac{k_{T}}{\rho c_{p}}
$$

Thermal diffusivity compares the ability of a material to conduct energy relative to its ability to store energy, i.e. it defines the velocity of heat transfer. In a material with small thermal diffusivity there is a slow change of temperature, while in a material with high thermal diffusivity the heat is rapidly transferred because of a high thermal conductivity with relation to its volumetric heat capacity $\beta$, defined by the denominator $\rho c_{p}$. 


\section{Radiation}

The thermal radiation is an electromagnetic radiation emitted by a body because of its temperature and internal energy. Contrary to the thermal conduction, the radiation does not require a medium to transfer energy and is even more efficiently in vacuum.

The calculation of thermal radiation is based on the Stefan-Boltzmann law, which relates the energy flux radiated by a body to the fourth power of the absolute temperature by

$$
e=\epsilon \sigma T^{4}
$$

where $e$ in $\mathrm{W} \cdot \mathrm{m}^{-2}$ is the surface specific emission, $\sigma=5.669 \times 10^{-8}$ $\mathrm{W} \cdot \mathrm{m}^{-2} \cdot \mathrm{K}^{-4}$ is the Stefan-Boltzmann constant and $\epsilon$ is the emissivity of a material, which corresponds to the relative ability of its surface to emit energy by radiation. Emissivity has values between $\epsilon=0$ (no emission) and $\epsilon=1$ (black body), i.e. for water $\epsilon=0.98$.

In case two bodies exchange heat by radiation, the net heat exchange is proportional to the difference in $T^{4}$. The net heat exchange from body 1 to body 2 is given by

$$
Q_{\text {rad }}=e_{S_{1}}-e_{S_{2}}=\epsilon \sigma S_{1}\left(T_{1}^{4}-T_{2}^{4}\right)
$$

where $S_{1}$ represents the contact surface. In most of the cases, at ambient temperatures $\left(\approx 20^{\circ} \mathrm{C}\right)$, the heat exchange produced by radiation is negligible in comparison with the heat exchange produced by convection.

\section{Convection}

Convective heat transfer is referred to the transfer of heat from a bounding surface to a flowing fluid. If the flow is induced by a device (e.g. a blower) or by a moving object the process is known as a forced convection. If the flow occurs as a result of the density difference produced by a temperature difference, the process is called free or natural convection.

The heat transfer through convection per unit surface is related by the Newton's law of cooling, which equation can be expressed as:

$$
Q_{h_{T}}=h_{T} S\left(T_{S}-T_{\infty}\right)
$$

where $h_{T}$ is the convective heat transfer coefficient, $T_{S}$ is the surface temperature and $T_{\infty}$ is the fluid temperature. 


\begin{tabular}{ll}
\hline Free convection Air & $5-25\left(\mathrm{~W} \cdot \mathrm{m}^{-2} \cdot \mathrm{K}^{-1}\right)$ \\
Free convection Water & $20-100\left(\mathrm{~W} \cdot \mathrm{m}^{-2} \cdot \mathrm{K}^{-1}\right)$ \\
Forced convection Air & $10-200\left(\mathrm{~W} \cdot \mathrm{m}^{-2} \cdot \mathrm{K}^{-1}\right)$ \\
Forced convection Water & $50-10.000\left(\mathrm{~W} \cdot \mathrm{m}^{-2} \cdot \mathrm{K}^{-1}\right)$ \\
Boiling Water & $3.000-100.000\left(\mathrm{~W} \cdot \mathrm{m}^{-2} \cdot \mathrm{K}^{-1}\right)$ \\
Condensing Water Vapour & $5.000-100.000\left(\mathrm{~W} \cdot \mathrm{m}^{-2} \cdot \mathrm{K}^{-1}\right)$ \\
\hline
\end{tabular}

Table 3.1. Convective heat transfer coefficient ranges.

The convection heat transfer coefficient is dependent on the type of media, i.e. gas or liquid, the flow properties such as velocity, viscosity and other flow and temperature dependent properties (see Appendix A for the information about $h_{T}$ in forced convection calculation).

Table 3.1 shows the convective heat transfer coefficient values for some common fluids.

From the heat transfer concept, this introduction leads to the following chapter structure. Section 3.2 describes the heat equation. Then, Section 3.3 gives the method for the solution of the heat equation. Afterwards the boundary conditions for the heat diffusion problems are explained in Section 3.4. The implementation of the heat model is presented in Section 3.5 and validated in Section 3.6. Finally the comparison of the results and some conclusions are detailed in Section 3.7.

\subsection{The Heat Equation}

The heat equation encompasses all the heat transfer mechanisms to evaluate the energy transfer between two bodies. Figure 3.1 represents, as a main example of this manuscript, the factors that dictate the energy transfer between a drop falling through a microwave cavity and the involved media. 


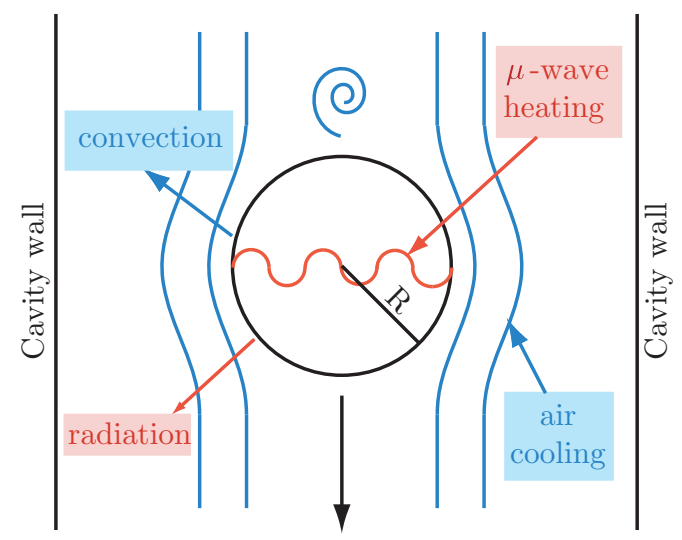

Figure 3.1. Main thermal factors that influence the heating of the droplet falling in the cavity.

\subsubsection{Definition}

A differential statement of the thermal energy balance defines the heat equation. Either when energy is removed or added through the boundaries of a body and/or energy is generated or absorbed within a body thermal energy is exchanged. The rate of change per unit volume $\dot{H}\left(\mathrm{~W} \cdot \mathrm{m}^{-3}\right)$ at a point inside a volume is

$$
\dot{H}=\frac{\partial H}{\partial t}
$$

where $H$ is the enthalpy or total energy per unit volume in $\mathrm{J} \cdot \mathrm{m}^{-3}$. The enthalpy of a body when changing its temperature from $T_{i}$ to $T_{f}$ is expressed from thermodynamics as:

$$
H=\int_{T_{i}}^{T_{f}} \rho c_{p} d T
$$

Then the rate of change of thermal energy becomes

$$
\dot{H}=\rho c_{p} \frac{\partial T}{\partial t}
$$

Since the heat leaving the surface of a material should be equal to that produce in the materials volume, for an isotropic material the Fourier energy 
balance equation $[49,50]$ allows the calculation of the temperature diffusion inside the material from the following equation:

$$
\rho \cdot c_{p} \frac{\partial T}{\partial t}=\nabla \cdot\left(k_{T} \cdot \nabla T\right)+Q_{n e t}
$$

where the term on the left side of the equation represents the energy stored as heat, the first term on the right is the Fourier net heat input by conduction and $Q_{\text {net }}\left(\mathrm{W} \cdot \mathrm{m}^{-3}\right)$ represents the net rate of heat generation in the material volume.

$$
Q_{n e t}=Q_{g e n}+Q_{\text {loss }}+Q_{\text {local }}+Q_{p h}
$$

$Q_{n e t}$ depends on the electromagnetic and thermal boundary conditions and can be solved by computing the electric field distribution within the sample in order to determine the microwave heat generation rate $\left(Q_{g e n}\right)$, the loss rate referred to the surface convection and radiation of energy $\left(Q_{\text {loss }}\right)$, the heat rate generated by any local or chemical reaction $\left(Q_{\text {local }}\right)$ and that derivated from phase changes or material transformation $\left(Q_{p h}\right)$. $Q_{\text {gen }}$ value is null in conventional heating, where the material is heated by contact at the surface according to the thermal boundary conditions. The microwave induced heating rate is given by [49]:

$$
Q_{g e n}=\omega \varepsilon_{0} \varepsilon^{\prime \prime}\left|E_{r m s}\right|^{2}
$$

where $\omega=2 \pi f_{0}$ is given by the resonant frequency $f_{0}(\mathrm{~Hz})$ of the cavity, $E_{r m s}$ is the electric field $\left(\mathrm{V} \cdot \mathrm{m}^{-1}\right)$ supplied to the cavity by an external generator, $\varepsilon_{0}$ is the vacuum permittivity $\left(\mathrm{F} \cdot \mathrm{m}^{-1}\right)$ and $\varepsilon^{\prime \prime}$ is the loss factor of the material (workload).

\subsubsection{Common Forms of the Heat Equation}

The commonly used equations representing the energy transfer are as follows: 
- Rectangular coordinates $(x, y, z)$ :

$$
\begin{aligned}
& \rho c_{p}\left(\frac{\partial T}{\partial t}+u \frac{\partial T}{\partial x}+v \frac{\partial T}{\partial y}+w \frac{\partial T}{\partial z}\right) \\
& =k_{T}\left(\frac{\partial^{2} T}{\partial x^{2}}+\frac{\partial^{2} T}{\partial y^{2}}+\frac{\partial^{2} T}{\partial z^{2}}\right)+2 \mu\left\{\left(\frac{\partial u}{\partial x}\right)^{2}+\left(\frac{\partial v}{\partial y}\right)^{2}+\left(\frac{\partial w}{\partial z}\right)^{2}\right\} \\
& +\mu\left\{\left(\frac{\partial u}{\partial y}+\frac{\partial v}{\partial x}\right)^{2}+\left(\frac{\partial u}{\partial z}+\frac{\partial w}{\partial x}\right)^{2}+\left(\frac{\partial v}{\partial z}+\frac{\partial w}{\partial y}\right)^{2}\right\}
\end{aligned}
$$

- Cylindrical coordinates $(r, \theta, z)$ :

$$
\begin{aligned}
\rho c_{p} & \left(\frac{\partial T}{\partial t}+v_{r} \frac{\partial T}{\partial r}+\frac{v_{\theta}}{r} \frac{\partial T}{\partial \theta}+v_{z} \frac{\partial T}{\partial z}\right) \\
& =k_{T}\left(\frac{1}{r} \frac{\partial}{\partial r}\left(r \frac{\partial T}{\partial r}\right)+\frac{1}{r^{2}} \frac{\partial^{2} T}{\partial \theta^{2}}+\frac{\partial^{2} T}{\partial z^{2}}\right) \\
& +2 \mu\left\{\left(\frac{\partial v_{r}}{\partial r}\right)^{2}+\left[\frac{1}{r}\left(\frac{\partial v_{\theta}}{\partial \theta}+v_{r}\right)\right]^{2}+\left(\frac{\partial v_{z}}{\partial z}\right)^{2}\right\} \\
& +\mu\left\{\left(\frac{\partial v_{\theta}}{\partial z}+\frac{1}{r} \frac{\partial v_{z}}{\partial \theta}\right)^{2}+\left(\frac{\partial v_{z}}{\partial r}+\frac{\partial v_{r}}{\partial z}\right)^{2}+\left[\frac{1}{r} \frac{\partial v_{r}}{\partial \theta}+r \frac{\partial}{\partial r}\left(\frac{v_{\theta}}{r}\right)\right]^{2}\right\}
\end{aligned}
$$

- Spherical coordinates $(r, \theta, \phi)$ :

$$
\begin{aligned}
& \rho c_{p}\left(\frac{\partial T}{\partial t}+v_{r} \frac{\partial T}{\partial r}+\frac{v_{\theta}}{r} \frac{\partial T}{\partial \theta}+\frac{v_{\theta}}{r \sin \theta} \frac{\partial T}{\partial \phi}\right) \\
& =k_{T}\left(\frac{1}{r^{2}} \frac{\partial}{\partial r}\left(r^{2} \frac{\partial T}{\partial r}\right)+\frac{1}{r^{2} \sin \theta} \frac{\partial}{\partial \theta}\left(\sin \theta \frac{\partial T}{\partial \theta}\right)+\frac{1}{r^{2} \sin ^{2} \theta} \frac{\partial^{2} T}{\partial \phi^{2}}\right) \\
& +2 \mu\left\{\left(\frac{\partial v_{r}}{\partial r}\right)^{2}+\left(\frac{1}{r} \frac{\partial v_{\theta}}{\partial \theta}+\frac{v_{r}}{r}\right)^{2}+\left(\frac{1}{r \sin \theta} \frac{\partial v_{\theta}}{\partial \phi}+\frac{v_{r}}{r}+\frac{v_{\theta} \cot \theta}{r}\right)^{2}\right\} \\
& +\mu\left\{\left[r \frac{\partial}{\partial r}\left(\frac{v_{\theta}}{r}\right)+\frac{1}{r} \frac{\partial v_{r}}{\partial \theta}\right]^{2}+\left[\frac{1}{r \sin \theta} \frac{\partial v_{r}}{\partial \phi}+r \frac{\partial}{\partial r}\left(\frac{v_{\phi}}{r}\right)\right]^{2}\right. \\
& \left.+\left[\frac{\sin \theta}{r} \frac{\partial}{\partial \theta}\left(\frac{v_{\phi}}{\sin \theta}\right)+\frac{1}{r \sin \theta} \frac{\partial v_{\theta}}{\partial \phi}\right]^{2}\right\}
\end{aligned}
$$


where the terms contained in braces \{\} are neglected because they are associated with viscous dissipation in systems with large velocity gradients.

In this manuscript the Fourier energy balance equation is reproduced simplifying the expressions to one dimensional model (because of the radial symmetry of the droplets) and complementing the equations adding the value of the net production of heat in the material volume $Q_{n e t}$.

\subsection{Heat Equation Solution by Numerical Methods}

Heat conduction problems are often solved by numerical mathematical models. The finite difference (FD) and finite elements (FE) are the main approximation techniques to solve the heat equation as an algebraic problem after a previous discretization (the medium is discretized producing a mesh of cells or volumes of finite size).

\subsubsection{Finite Difference Time Domain Method}

A Finite Difference gives a function information with a discrete approximation. There are two kinds of FD, implicit when solving an equations system is required; and explicit when a time loop is generated and the new values are calculated from previous values. The case presented here is explicit and it provides the necessary imput for in the calculation of the temperature evolution with time when a material is heated by microwaves.

It is important to consider that when an explicit Finite Difference Time Domain (FDTD) is applied to solve an equation, instability problems may occur. That is the reason why the discrete time interval $(\Delta t)$ must be dimensioned according to the chosen discrete space interval $(\Delta z)$, i.e. the transit time between two nodes $(\Delta z)$ must be higher that the chosen time step $(\Delta t)$.

$$
\Delta t \leq \frac{\Delta z}{c}
$$

where $c \approx 3 \times 10^{8} \mathrm{~m} \cdot \mathrm{s}^{-1}$ is the speed of light. The microwave energy balance equation is discretized using a FDTD method to study the temperature evolution. As aforesaid the equation is simplified to a one-dimensional model because of the radial symmetry and it is developed in three different coordinates systems. 
a)

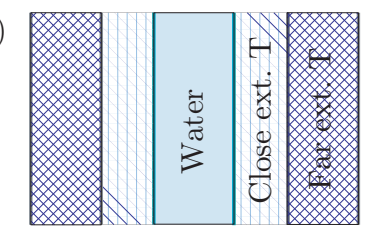

b)

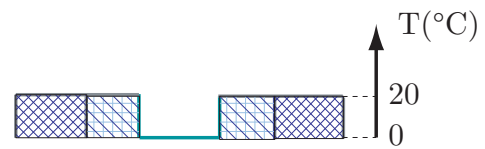

c)

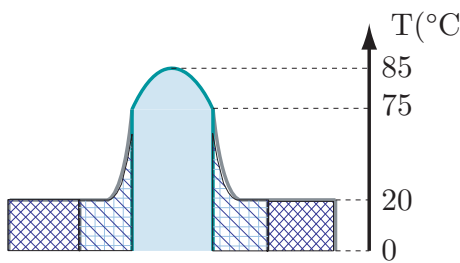

Figure 3.2. Representation of the rectangular thermal case: a) 1D drop rectangular profile. b) Temperature profile before heating and c) after heating. The drop is initially cooled, therefore the low water-temperature value in $b$ )

The rectangular case represents the case of a material filling the entire volume of a rectangular microwaves cavity which extends infinitely in two directions. As seen in Figure 3.2 in the rectangular case the material is enclosed between two air walls representing the external boundaries. The temperature is calculated over a spatial vector where each point is defined as external or internal of the material being studied.

The cylindrical coordinates system analyses the temperature evolution of the cylinder formed when a liquid material flows through the central axis of a microwaves cavity. Finally, the spherical coordinate system represents the temperature evolution of a single drop inside a microwave cavity.

The temperature rise in the material can be calculated combining Equation 3.11 and Equations 3.14, 3.15 and 3.16, and simplifying them to a single partial differential equation:

- Rectangular coordinates $(z)$ :

$$
\frac{\partial T}{\partial t}=\left(\frac{k_{T}}{\rho c_{p}}\right) \frac{\partial^{2} T}{\partial z^{2}}+\frac{1}{\rho c_{p}} Q_{g e n}
$$


- Cylindrical coordinates $(r)$ :

$$
\frac{\partial T}{\partial t}=\left(\frac{k_{T}}{\rho c_{p}}\right) \frac{1}{r} \frac{\partial}{\partial r}\left(r \frac{\partial T}{\partial r}\right)+\frac{1}{\rho c_{p}} Q_{g e n}
$$

- Spherical coordinates $(r)$ :

$$
\frac{\partial T}{\partial t}=\left(\frac{k_{T}}{\rho c_{p}}\right) \frac{1}{r^{2}} \frac{\partial}{\partial r}\left(r^{2} \frac{\partial T}{\partial r}\right)+\frac{1}{\rho c_{p}} Q_{g e n}
$$

The Crank-Nicholson strategy [51, 52], often used in diffusion problems, is applied to discretize these partial differential equations. A linear interpolation of the neighbour node values computes the first order spatial derivative over the same time step $n+1$ (seen in 3.21). Equation 3.22 shows how the first order time partial derivative is calculated by a linear interpolation of the adjacent time steps over the same spatial node $i$.

$$
\begin{aligned}
& \frac{\partial T}{\partial z}=\frac{T_{i+1}^{n+1}-T_{i}^{n+1}}{\Delta z} \\
& \frac{\partial T}{\partial t}=\frac{T_{i}^{n+1}-T_{i}^{n}}{\Delta t}
\end{aligned}
$$

Finally, the second order spatial derivative is substituted in Equation 3.23 by a linear interpolation of the adjacent nodes to the one being considered, and enables the time dependence between two continuous instants ( $n$ and $n+1)$.

$$
\frac{\partial^{2} T}{\partial z^{2}}=\frac{1}{2}\left[\frac{T_{i-1}^{n+1}-2 T_{i}^{n+1}+T_{i+1}^{n+1}}{\Delta z^{2}}+\frac{T_{i-1}^{n}-2 T_{i}^{n}+T_{i+1}^{n}}{\Delta z^{2}}\right]
$$

Combining the energy Equations 3.18, 3.19 and 3.20 and the Crank-Nicholson strategy Equations 3.21, 3.22 and 3.23 the temperature profile can be obtained in each time step (see Appendix B for a complete Crank-Nicholson explanation and the development followed to obtain the next expressions).

- Rectangular coordinates $(z)$ :

$$
\frac{T_{i}^{n+1}-T_{i}^{n}}{\Delta t}=\alpha \frac{1}{2}\left[\frac{T_{i-1}^{n+1}-2 T_{i}^{n+1}+T_{i+1}^{n+1}}{\Delta z^{2}}+\frac{T_{i-1}^{n}-2 T_{i}^{n}+T_{i+1}^{n}}{\Delta z^{2}}\right]+\beta g_{i}^{n}
$$


- Cylindrical coordinates $(r)$ :

$$
\begin{aligned}
& \frac{T_{i}^{n+1}-T_{i}^{n}}{\Delta t}=\alpha \frac{1}{2}\left[\frac{1}{r} \frac{T_{i+1}^{n+1}-T_{i-1}^{n+1}}{\Delta r}+\frac{T_{i-1}^{n+1}-2 T_{i}^{n+1}+T_{i+1}^{n+1}}{\Delta r^{2}}\right. \\
& \left.+\frac{T_{i-1}^{n}-2 T_{i}^{n}+T_{i+1}^{n}}{\Delta r^{2}}\right]+\beta g_{i}^{n}
\end{aligned}
$$

- Spherical coordinates $(r)$ :

$$
\begin{aligned}
& \frac{T_{i}^{n+1}-T_{i}^{n}}{\Delta t}=\alpha\left[\frac{1}{r} \frac{T_{i+1}^{n+1}-T_{i-1}^{n+1}}{\Delta r}+\frac{1}{2}\left(\frac{T_{i-1}^{n+1}-2 T_{i}^{n+1}+T_{i+1}^{n+1}}{\Delta r^{2}}\right.\right. \\
& \left.\left.+\quad \frac{T_{i-1}^{n}-2 T_{i}^{n}+T_{i+1}^{n}}{\Delta r^{2}}\right)\right]+\beta g_{i}^{n}
\end{aligned}
$$

The constants are regrouped using the thermal diffusivity $\alpha$ (eq. 3.4) and the volumetric heat capacity $\beta$ concepts.

Separating the terms in the $n+1$ and the $n$ instant, it is possible to find a general equation for the three coordinate systems in matrix form:

$$
\left(\mathbf{I}-\frac{1}{2} c_{1} \mathbf{A}-\frac{1}{2} c_{2} \mathbf{B}\right) \mathbf{t}^{n+1}=\left(\mathbf{I}+\frac{1}{2} c_{1} \mathbf{A}\right) \mathbf{t}^{n}+\frac{c_{1}}{2}\left(\mathbf{b}^{n+1}+\mathbf{b}^{n}\right)+\mathbf{s}^{n}
$$

Where the constants, matrices and vectors are defined as follows:

$$
\begin{aligned}
& \mathbf{s}^{n}=\Delta t \beta \mathbf{g}^{n}, \\
& c_{1}=\frac{\alpha \Delta t}{\Delta z^{2}}, c_{2}=\frac{\alpha \Delta t}{\Delta r} \frac{1}{r}(\delta-1)\left\{\begin{array}{l}
\delta=1 \\
\delta=2 \\
\delta=3 \\
\text { (rectangular coordinates) } \\
\text { (spherical coordinates) }
\end{array}\right. \\
& \mathbf{A}=\left(\begin{array}{ccccc}
-2 & 1 & 0 & \cdots & 0 \\
1 & -2 & 1 & \ddots & \vdots \\
0 & \vdots & \vdots & \vdots & 0 \\
\vdots & \ddots & 1 & -2 & 1 \\
0 & \cdots & 0 & 1 & -2
\end{array}\right) \mathbf{B}=\left(\begin{array}{ccccc}
0 & 1 & 0 & \cdots & 0 \\
-1 & 0 & 1 & \ddots & \vdots \\
0 & \vdots & \vdots & \vdots & 0 \\
\vdots & \ddots & -1 & 0 & 1 \\
0 & \cdots & 0 & -1 & 0
\end{array}\right)
\end{aligned}
$$




$$
\mathbf{t}^{n}=\left(\begin{array}{c}
T_{1}^{n} \\
T_{2}^{n} \\
\vdots \\
T_{N-1}^{n} \\
T_{N}^{n}
\end{array}\right) \mathbf{t}^{n+1}=\left(\begin{array}{c}
T_{1}^{n+1} \\
T_{2}^{n+1} \\
\vdots \\
T_{N-1}^{n+1} \\
T_{N}^{n+1}
\end{array}\right) \mathbf{b}^{n}=\left(\begin{array}{c}
b_{0}^{n} \\
0 \\
\vdots \\
0 \\
b_{N+1}^{n}
\end{array}\right) \mathbf{g}^{n}=\left(\begin{array}{c}
g_{1}^{n} \\
g_{2}^{n} \\
\vdots \\
g_{N-1}^{n} \\
g_{N}^{n}
\end{array}\right)
$$

Depending on which boundary conditions of the material are applied to the study, the matrix $\mathbf{A}$ and the vector $\mathbf{b}$ will be changed adjusting the model to these conditions. The vector $\mathbf{g}$ changes depending on the features of the microwaves cavity and the heating generation rate $Q_{g e n}$.

\subsection{Boundary Conditions}

It is important to consider the external conditions (heat, mass and pressure transfer) at the surface of the material that is being heated by microwaves. The surrounding could be air or a different material.

\subsubsection{The Dirichlet Boundary Condition}

The first type of boundary condition (BC) defining the values of a function on the surface or border is the Dirichlet boundary condition.

Applied to the heat equation, the isothermal (Dirichlet) BC gives the information about the temperature distribution on the surface of the heated material, which could be considered constant.

The outer points of the vector $\mathbf{b}$ described before are fixed to a specified temperature (i.e. $b_{0}^{n}=T_{0}^{n}$ and $b_{N+1}^{n}=T_{N+1}^{n}$ ).

\subsubsection{The von Neumann Boundary Condition}

In the case of a von Neumann boundary condition the temperature distribution at the boundaries is calculated with the evaluation of the surrounding air effect. These boundary conditions are directly related with the convection mechanism, which is defined as follows:

$$
-k_{T}\left[\frac{\partial T}{\partial n}\right]_{S}=h_{T}\left(T_{S}-T_{\infty}\right)
$$


where $n$ is the coordinate normal to the heated surface, $h_{T}$ is the convection heat transfer coefficient $\left(\mathrm{W} \cdot \mathrm{m}^{-2} \cdot \mathrm{K}^{-1}\right)$ computed according the description in Appendix A, $T_{S}$ is the surface temperature (in $\mathrm{K}$ ) and $T_{\infty}$ is the environmental temperature (in $\mathrm{K}$ ). In the case of conduction heat transfer boundary between materials of different thermal conductivities the next equation is used:

$$
k_{T 1}\left[\frac{\partial T_{1}}{\partial n}\right]_{S}=k_{T 2}\left[\frac{\partial T_{2}}{\partial n}\right]_{S}
$$

where $k_{T 1}$ and $T_{1}$ are respectively the thermal conductivity and surface temperature of one layer and $k_{T 2}$ and $T_{2}$ are the thermal conductivity and surface temperature of the other layer.

The Neumann boundary conditions are the ones selected for the designed model because of the air that surrounds the material when it is inside the cavity.

The approach to calculate the Newton-Neumann boundary conditions is based on the idea of using a Dirichlet BC that will embody the von Neumann BC.

The material is discretized as a vector where the first point is $z_{0}=0$ and the last point is the length (dimension) of the material $z_{N+1}=L$. The discrete space interval is given by the length and the number of points used.

$$
\Delta z=\frac{L}{N+1}
$$

The value of $T$ for $0<z<L$ is known since it is in the bulk:

$$
T(z, 0)=f(z)
$$

that means that it is possible to calculate the differential equation defined in Section 3.3.1 for all the points $\left(T_{1}, T_{2}, \cdots, T_{N}\right)$. With them it is possible to get the values at the borders. The two external points are added to the vector representing the material: in the first position $T(0,1)$ and in the final position $T(L, 1)$. For the boundary values the first derivative of the point is imposed. In case of no losses the condition is:

$$
\begin{aligned}
& \frac{\partial T(0,1)}{\partial z}=0 \\
& \frac{\partial T(L, 1)}{\partial z}=0
\end{aligned}
$$


while in case of losses due to convection the condition is:

$$
\begin{aligned}
& \frac{\partial T(0,1)}{\partial z}=k_{1} T(0,1)+\beta_{1} \\
& \frac{\partial T(L, 1)}{\partial z}=k_{2} T(L, 1)+\beta_{2}
\end{aligned}
$$

where the constant values are calculated using Equation 3.25.

$$
\begin{aligned}
& k_{1}=-\frac{h_{T}}{k_{T}}, k_{2}=\frac{h_{T}}{k_{T}} \\
& \beta_{1}=\beta_{2}=\frac{h_{T}}{k_{T}} T_{\infty}
\end{aligned}
$$

To calculate the values $T_{0}$ and $T_{N+1}$ a Taylor expansion of $T$ around $z_{0}$ and $z_{N+1}$ up to second order is performed. For the case of the $z_{0}$ boundary (the $z_{N+1}$ boundary is calculated following the same procedure) it follows that:

$$
\begin{aligned}
& T\left(z_{0}+\Delta z\right)=T\left(z_{0}\right)+\Delta z T\left(z_{0}\right)^{\prime}+\frac{\Delta z^{2}}{2} T\left(z_{0}\right)^{\prime \prime}+\vartheta\left(\Delta z^{3}\right) \\
& T\left(z_{0}+2 \Delta z\right)=T\left(z_{0}\right)+2 \Delta z T\left(z_{0}\right)^{\prime}+2 \Delta z^{2} T\left(z_{0}\right)^{\prime \prime}+\vartheta\left(\Delta z^{3}\right)
\end{aligned}
$$

For $\Delta z=1$ :

$$
\begin{aligned}
& T_{1}=T_{0}+\Delta z T_{0}^{\prime}+\frac{\Delta z^{2}}{2} T_{0}^{\prime \prime}+\vartheta\left(\Delta z^{3}\right) \\
& T_{2}=T_{0}+2 \Delta z T_{0}^{\prime}+2 \Delta z^{2} T_{0}^{\prime \prime}+\vartheta\left(\Delta z^{3}\right)
\end{aligned}
$$

where the values $T_{1}$ and $T_{2}$ are known. From the system it is possible to get $T_{0}^{\prime}$ :

$$
T_{0}^{\prime}=\frac{1}{2 \Delta z}\left(4 T_{1}-T_{2}-3 T_{0}\right)
$$

and imposing the condition $3.28 T_{0}$ is solved as:

$$
T_{0}=\frac{4}{3+2 \Delta z k_{1}} T_{1}-\frac{1}{3+2 \Delta z k_{1}} T_{2}-\frac{2 \Delta z \beta_{1}}{3+2 \Delta z k_{1}}
$$

The solution has a structure of type

$$
\begin{aligned}
& T_{0}=p_{1} T_{1}+p_{2} T_{2}+p_{3} \\
& T_{N+1}=q_{1} T_{1}+q_{2} T_{2}+q_{3}
\end{aligned}
$$


where $p_{1}, p_{2}, p_{3}, q_{1}, q_{2}$ and $q_{3}$ should be coefficients to satisfy the boundary conditions for the order of the chosen method.

In case of $T_{0}$ the coefficients $p_{1}$ and $p_{2}$ will be in the first row of the matrix $\mathbf{A}$ and the coefficient $p_{3}$ with $b_{0}$. In case of $T_{N+1}$ the coefficients $q_{1}$ and $q_{2}$ will be in the last row of the matrix $\mathbf{A}$ and the coefficient $q_{3}$ is $b_{N+1}$.

\subsection{Implementation and Results}

The heat equation described in Sections 3.3 and 3.4 is implemented following the FDTD description adjusted to the BC using MATLAB ${ }^{\circledR}$.

A resume of the code is seen in Algorithm 1, while Algorithms 2 and 3 offers a detailed definition split in two parts.

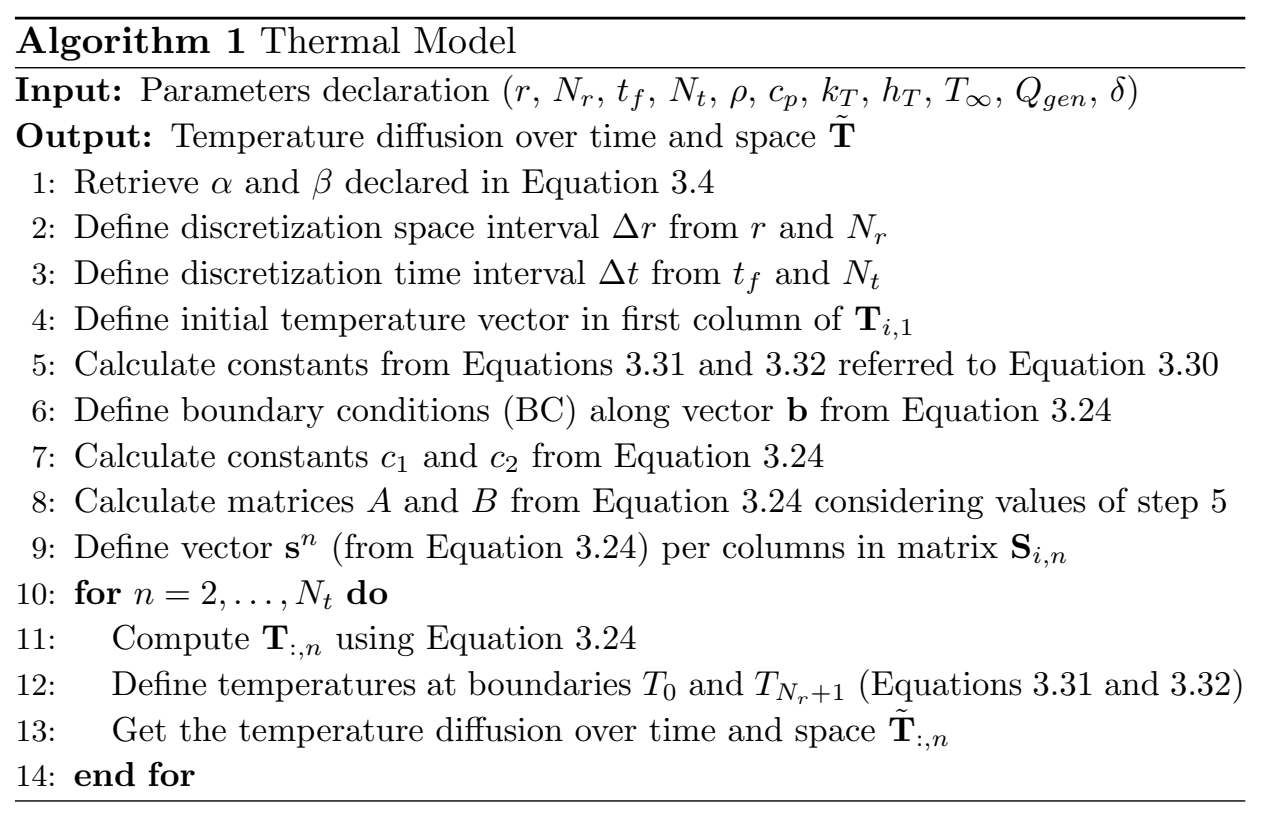

Some of the input parameters $\left(t_{f}, h_{T}\right.$ and $\left.Q_{g e n}\right)$ are given for this code as a fixed value. Those parameters are introduced in the next chapters and then, the algorithm is updated. 


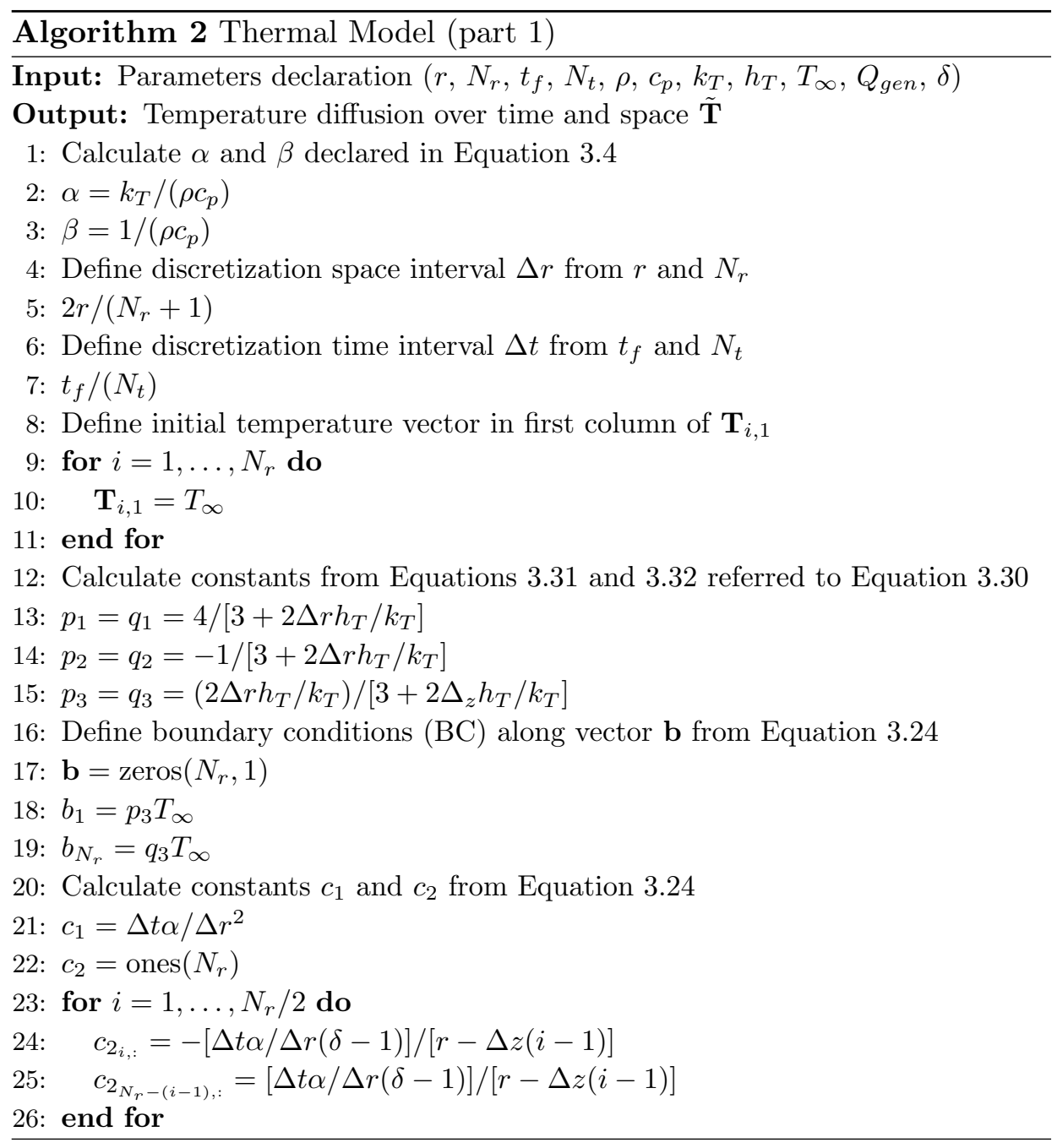




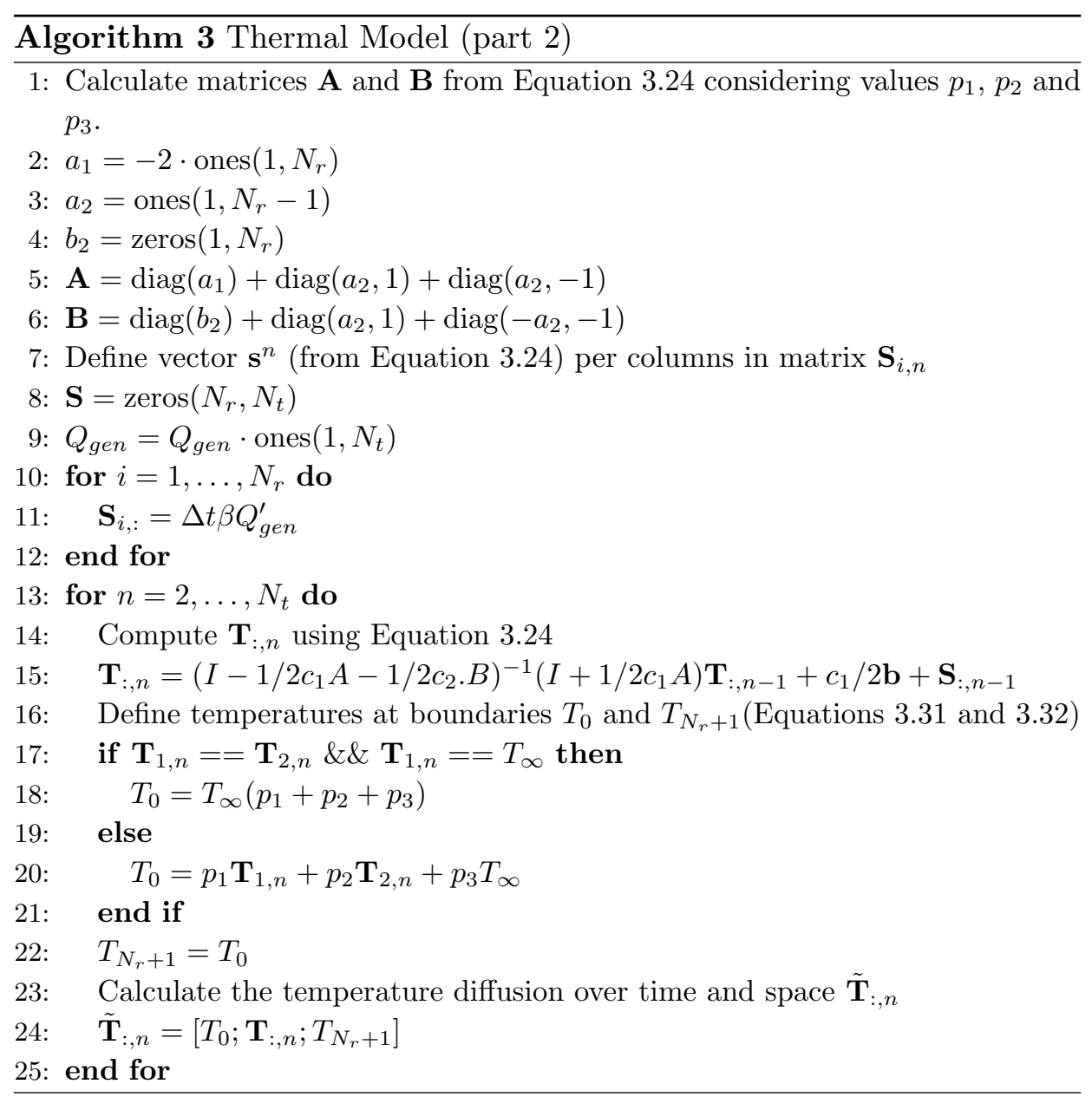

\subsubsection{Thermal Parameters Influence}

Several parameter studies are conducted in order to estimate the appropriate input power for the internal gelation. In all studies a $80 \mathrm{~K}$ temperature increase of the droplet center is targeted. This temperature shift is chosen in order to initiate on one hand the internal gelation (explained in Section 2.5.2) and to omit on the other hand an overheating of the center. The overheating of the droplet (leading to its explosion) is considered as the most critical aspect in the study. Since the center temperature is the highest in the droplet, it is taken as reference value. The working window for the internal gelation starts at $60{ }^{\circ} \mathrm{C}$. 


\begin{tabular}{ll}
\hline Density $(\rho)$ & $1000 \mathrm{~kg} \cdot \mathrm{m}^{-3}$ \\
Specific heat capacity $\left(c_{p}\right)$ & $4190 \mathrm{~J} \cdot \mathrm{kg}^{-1} \cdot \mathrm{K}^{-1}$ \\
Thermal conductivity $\left(k_{T}\right)$ & $0.6 \mathrm{~W} \cdot \mathrm{m}^{-1} \cdot \mathrm{K}^{-1}$ \\
\hline
\end{tabular}

Table 3.2. Common properties for water at atmospheric pressure.

The most relevant parameters (size of droplet, thermal conductivity, free convection heat transfer coefficient) are considered to determine the optimal microwave power and the frequency that should be used for the production. Water is used as a material in a first approximation. Its relevant parameters are shown in Table 3.2.

Using this thermal parameters, the microwave heat generation rate needed to heat multiple sizes of drops is calculated. As can be seen in Figure 3.3 , a lower $Q_{\text {gen }}$ is needed when the radius of the drop grows. Furthermore the value decreases with increasing processing time in the cavity.

This result is expected because the volume to surface ratio is increased with the radius and thus the relative heat losses through the surface decrease.

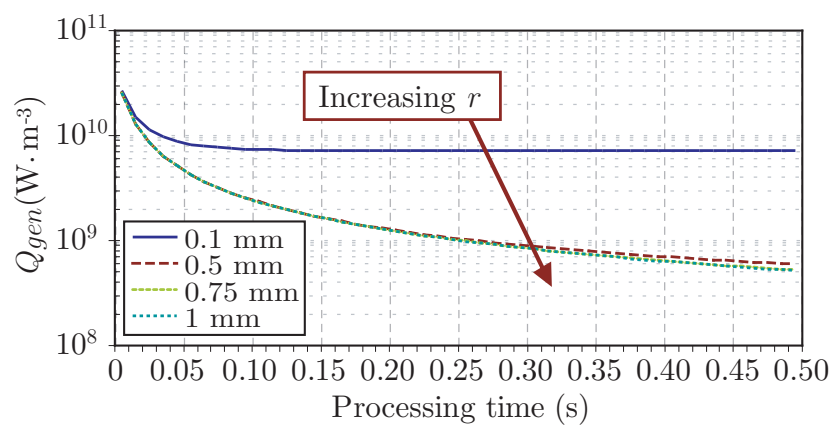

Figure 3.3. Microwave heat generation rate $\left(Q_{g e n}\right)$ as function of processing time for different drop radius, to achieve a temperature increase of $80 \mathrm{~K}$. 


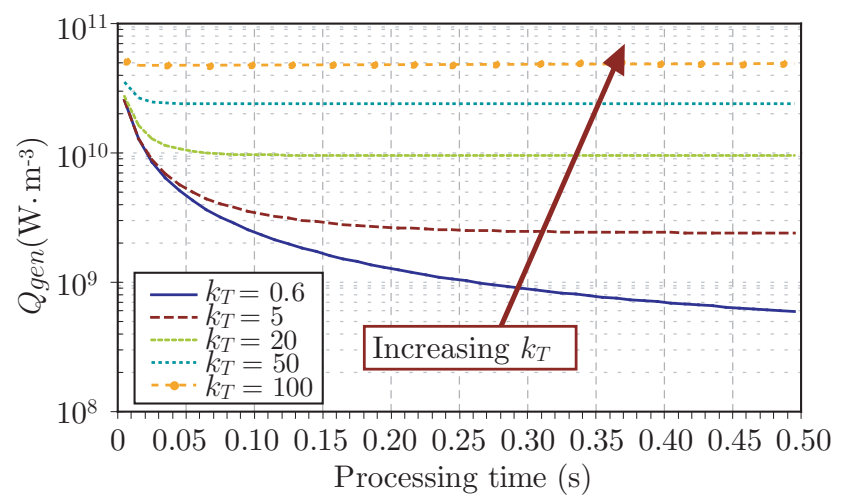

Figure 3.4. Microwave heat generation rate $\left(Q_{g e n}\right)$ as function of processing time for several values of thermal conductivity, to achieve a temperature increase of $80 \mathrm{~K}$ in a $0.5 \mathrm{~mm}$ radius drop.

The droplets planned to be used in the real experiments have a radius of $0.5 \mathrm{~mm}$. The influence of the thermal conductivity in the model is studied for this size, keeping the rest of the water thermal properties $\left(\rho, c_{p}\right)$. The higher the thermal conductivity is, the higher the thermal loss. Therefore, for a bigger $k_{T}$, a higher $Q_{g e n}$ is needed to heat the droplet (Figure 3.4).

Finally the convection heat transfer coefficient $\left(h_{T}\right)$ is varied to determine its effect on the model. As seen in Section 3.4, this coefficient introduce the relation of the material with its surrounding through the BC modifying the surface temperature. Then, considering the same direction of heating, this coefficient is independent of the droplet size. As can be seen in Figure 3.5, for a processing time of $92 \mathrm{~ms}$, the boundary temperature decreases with a $h_{T}$ increase. 


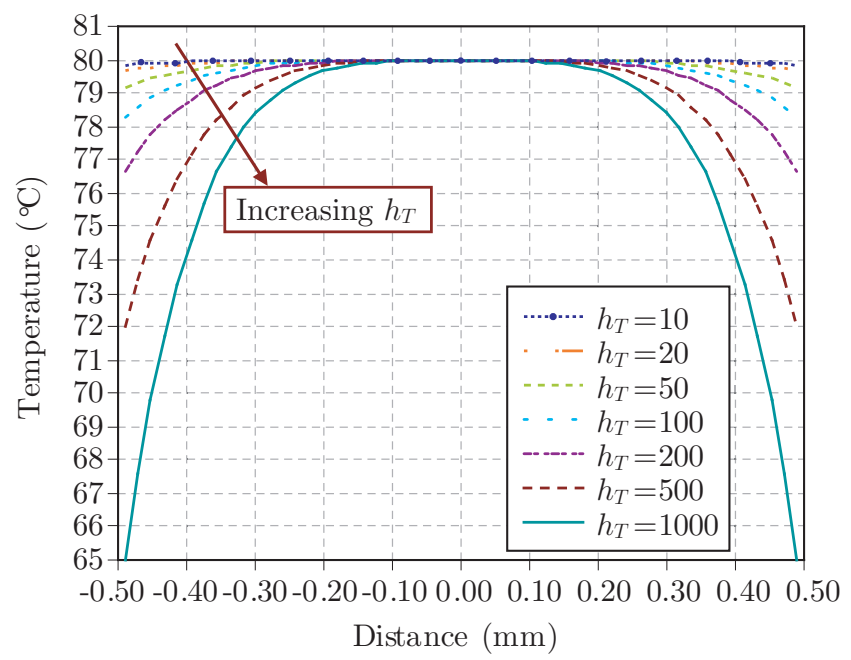

Figure 3.5. Temperature profile of a $0.5 \mathrm{~mm}$ water droplet for several values of convection heat transfer coefficient.

\subsubsection{Influence of the Coordinates System Election}

As an example the model is evaluated considering a $0.5 \mathrm{~mm}$ radius water drop (thermal properties in Table 3.2). The forced convection coefficient is supposed to be a fixed value $h_{T}=250 \mathrm{~W} \cdot \mathrm{m}^{-2} \cdot \mathrm{K}^{-1}$ and the environmental external temperature is considered $T_{\infty}=20^{\circ} \mathrm{C}$. The drop is heated by microwaves and the microwave heat generation rate is fixed to $Q_{g e n}=$ $10^{9} \mathrm{~W} \cdot \mathrm{m}^{-3}$ for the first calculations.

Figure 3.6 a) shows the temperature profile as function of the radial position inside the drop. Every line represents a different time step. In Figure $3.6 \mathrm{~b}$ ), in addition to the radial position dependence, there is a better appreciation of the temperature variation with time.

The processing time $t=100 \mathrm{~s}$ has been selected in order to bring the model to a temperature saturation value and to see the temperature deviation between the center point and the surface points of the drop, that in this case is $\Delta T=208.241 \mathrm{~K}$.

Figure 3.7 a) and b) present the temperature profile in cylindrical coordinates following the same structure as seen for the rectangular case. 

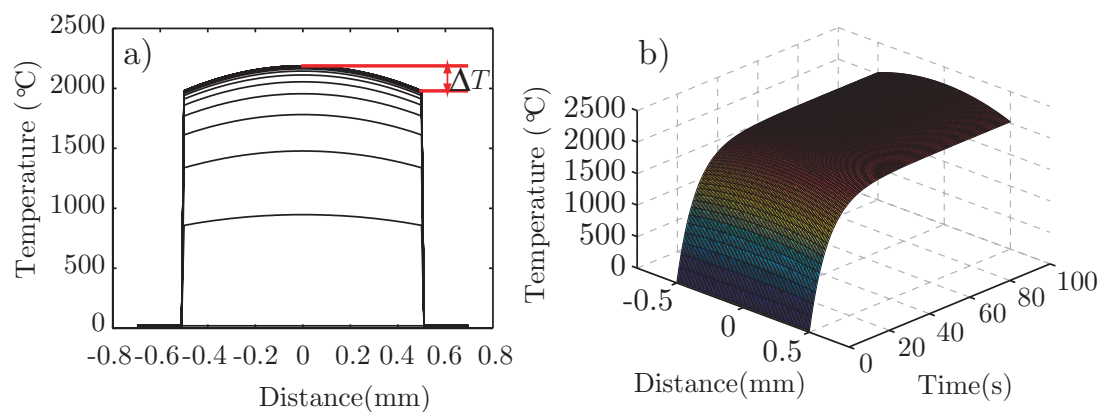

Figure 3.6. Temperature profile (in rectangular coordinates) of a drop heated by microwaves as function of a) radius, b) radius and processing time.
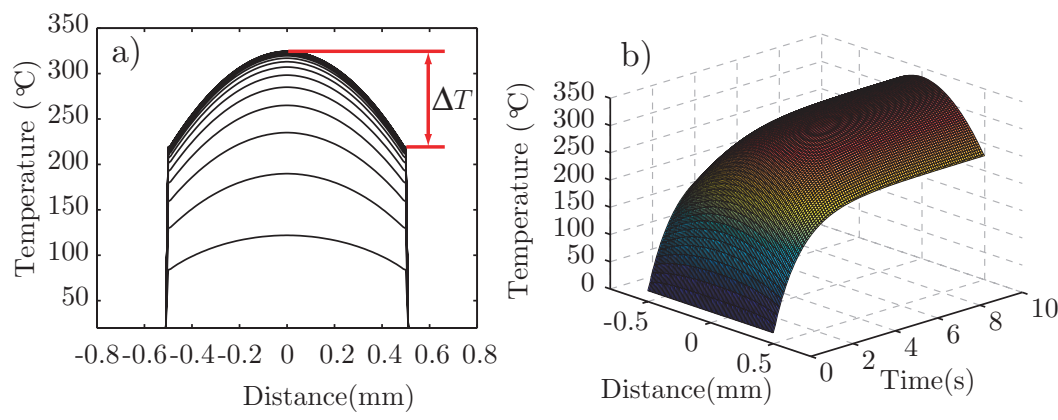

Figure 3.7. Temperature profile (in cylindrical coordinates) of a drop heated by microwaves as function of a) radius, b) radius and processing time.

The cylindrical model needs a lower processing time $t=10 \mathrm{~s}$ to reach the temperature saturation. The temperature deviation is here $\Delta T=$ $105.440 \mathrm{~K}$.

Figure 3.8 a) and b) present the temperature profile in spherical coordinates following the same structure as seen for the previous cases.

The processing time is chosen as for the cylindrical case $t=10 \mathrm{~s}$ and the temperature deviation is $\Delta T=70.228 \mathrm{~K}$.

In Figures 3.6, 3.7 and 3.8 it is possible to see that the temperature deviation is increasing from the case of rectangular coordinates to the case 

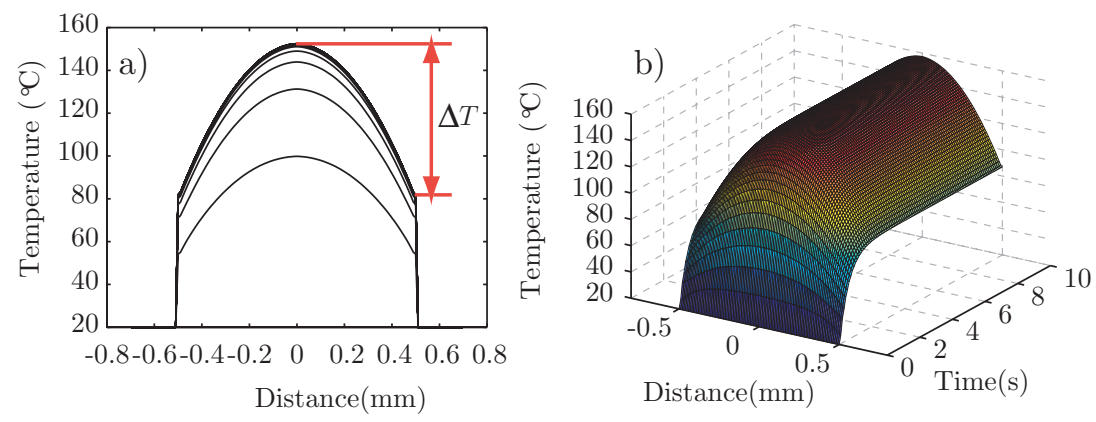

Figure 3.8. Temperature profile (in spherical coordinates) of a drop heated by microwaves as function of a) radius, b) radius and processing time.

of spherical coordinates, i.e. the temperature at the borders is lower in the spherical case. This is due to the lowest surface to volume ratio in case of a sphere.

\subsection{Validation}

Several validations are made to ensure the consistency of the FDTD thermal model. The first one consists on a theoretical validation, then a validation is made using the PDE Toolbox from Matlab software and finally a validation based on the reproduction of examples from the literature is presented.

\subsubsection{Physically Validation}

The physical validation is based on the concepts shown in Section 3.1. The principles of heat transfer are applied below to the three coordinates systems to know the maximal temperature deviation between the borders and the center of a material.

\section{Rectangular Coordinates}

An infinitesimal plate with $\Delta x$ high, $\Delta y$ width and $\Delta z$ thickness is considered (see Figure 3.9 a)).

By applying the second law of thermodynamics, the heating of the 


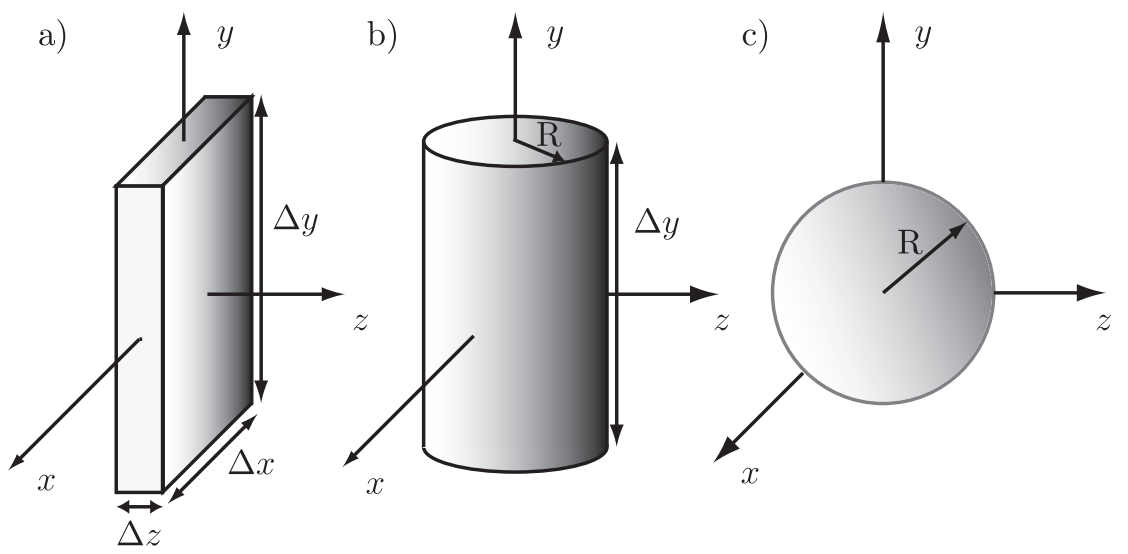

Figure 3.9. Infinitesimal volume geometry in rectangular coordinates $a$ ), cylindrical coordinates $b$ ) and spherical coordinates $c$ ).

plate volume should be the same as the heat leaving its surface:

$$
Q_{\text {gen }} \cdot \Delta x \cdot \Delta y \cdot \Delta z \cdot 2=Q_{\text {flux }} \cdot \Delta x \cdot \Delta y \cdot 2
$$

Then, depending on the plate $z$ position:

$$
Q_{\text {flux }}=Q_{\text {gen }} \cdot z
$$

The heat flow is related with the thermal conductivity by:

$$
Q_{\text {flux }}=-k_{T} \cdot \nabla T \Rightarrow Q_{\text {flux }}(z)=-k_{T} \frac{\mathrm{d} T}{\mathrm{~d} z}
$$

then:

$$
\mathrm{d} T=-\frac{1}{k_{T}} Q_{f l u x}(z) \mathrm{d} z=-\frac{1}{k_{T}} Q_{g e n} \cdot z \cdot \mathrm{d} z
$$

Finally, the temperature distribution follows the next relation depending on the $z$ position along the plate thickness:

$$
T(z)=\int_{T_{\text {center }}}^{T_{z}} \mathrm{~d} T=\frac{Q_{\text {gen }}}{k_{T}} \int_{0}^{z} z \cdot \mathrm{d} z=T_{\text {center }}-\frac{Q_{g e n} \cdot z^{2}}{2 \cdot k_{T}}
$$

Applying the same parameters as in the example presented in 3.5.2 $\left(Q_{g e n}=\right.$ $\left.10^{9} \mathrm{~W} \cdot \mathrm{m}^{-3}, r=0.5 \mathrm{~mm}, k_{T}=0.6 \mathrm{~W} \cdot \mathrm{m}^{-1} \cdot \mathrm{K}^{-1}\right)$ the temperature deviation between the center and the surface of the drop (considered as a plate) is $\Delta T=208.333 \mathrm{~K}$. 


\section{Cylindrical Coordinates}

In a given infinitesimal segment of a cylinder with radius $R$ and $\Delta y$ width (Figure $3.9 \mathrm{~b}$ )), the heat generated in the cylinder volume should be equal to the heat leaving its surface:

$$
Q_{\text {gen }} \cdot \pi \cdot R^{2} \cdot \Delta y=Q_{\text {flux }} \cdot 2 \cdot \pi \cdot R \cdot \Delta y
$$

Depending on the $r$ radial position within the cylinder:

$$
Q_{f l u x}(r)=\frac{Q_{g e n}(r) \cdot r}{2}
$$

The heat flow is related to the thermal conductivity by:

$$
Q_{f l u x}=-k_{T} \cdot \nabla T \Rightarrow Q_{f l u x}(r)=-k_{T} \frac{\mathrm{d} T}{\mathrm{~d} r}
$$

then:

$$
\mathrm{d} T=-\frac{1}{k_{T}} Q_{f l u x}(r) \mathrm{d} r=-\frac{1}{k_{T}} \frac{Q_{g e n}(r) \cdot r}{2} \cdot \mathrm{d} r
$$

The temperature distribution depending on the radial $r$ position within the cylinder is calculated by integration:

$$
T(r)=\int_{T_{\text {center }}}^{T_{r}} \mathrm{~d} T=\frac{Q_{g e n}}{2 \cdot k_{T}} \int_{0}^{r} r \cdot \mathrm{d} r=T_{\text {center }}-\frac{Q_{g e n} \cdot r^{2}}{4 \cdot k_{T}}
$$

For a radial position equal to the drop radius $r=R=0.5 \mathrm{~mm}$ (considered as a cylinder) and using the same thermal parameters as before $\left(Q_{g e n}, k_{T}\right)$, the maximal temperature deviation is now $\Delta T=104.167 \mathrm{~K}$.

\section{Spherical Coordinates}

In a given sphere of $R$ radius (Figure $3.9 \mathrm{c}$ )), the heating of the sphere volume should be the same as the heat leaving the surface:

$$
Q_{\text {gen }} \cdot \frac{4}{3} \cdot \pi \cdot R^{3}=Q_{\text {flux }} \cdot 4 \cdot \pi \cdot R^{2}
$$

Depending on the radial $r$ position within the sphere:

$$
Q_{f l u x}(r)=\frac{Q_{g e n}(r) \cdot r}{3}
$$


The heat flow is related with the thermal conductivity by:

$$
Q_{\text {flux }}=-k_{T} \cdot \nabla T \Rightarrow Q_{f l u x}(r)=-k_{T} \frac{\mathrm{d} T}{\mathrm{~d} r}
$$

then:

$$
\mathrm{d} T=-\frac{1}{k_{T}} Q_{f l u x}(r) \mathrm{d} r=-\frac{1}{k_{T}} \frac{Q_{g e n}(r) \cdot r}{3} \cdot \mathrm{d} r
$$

The temperature distribution depending on the radial $r$ position within the sphere is calculated by integration:

$$
T(r)=\int_{T_{\text {center }}}^{T_{r}} \mathrm{~d} T=\frac{Q_{g e n}}{3 \cdot k_{T}} \int_{0}^{r} r \cdot \mathrm{d} r=T_{\text {center }}-\frac{Q_{g e n} \cdot r^{2}}{6 \cdot k_{T}}
$$

A spherical drop of radius $R=0.5 \mathrm{~mm}$ has a maximal temperature deviation, between the surface and the center, of $\Delta T=69.444 \mathrm{~K}$ considering the same thermal parameters $\left(Q_{g e n}, k_{T}\right)$.

\subsubsection{Matlab Pdetools}

The Partial Differential Equation Toolbox is a software package that includes a complete graphical user interface (GUI) in Matlab and covers all aspects of a partial differential equation processes [53]. The tool includes several types of PDE application including electromagnetic modelling and the heat transfer equation, and solves the partial derivative equations using the Finite Elements Method FEM. These numerical methods help solving computational electromagnetic analysis problems, which motivate the use of pdetools as a validation of the designed thermal model.

Because pdetools is working with two dimensional geometry models, only the rectangular and the cylindrical model can be validated considering an infinitesimal rectangle and cylinder. The main idea of the FEM is the approximation of the computational domain with the union of simple geometric objects (i.e. finite elements) forming a mesh in the drawn geometry.

To validate the rectangular model, an infinitesimal rectangle is designed in pdetools (Figure $3.10 \mathrm{a}$ )). The main point of the design is that the dimension in which the heating is considered should be the same that the selected sphere radius in the implemented FDTD thermal model. In case 

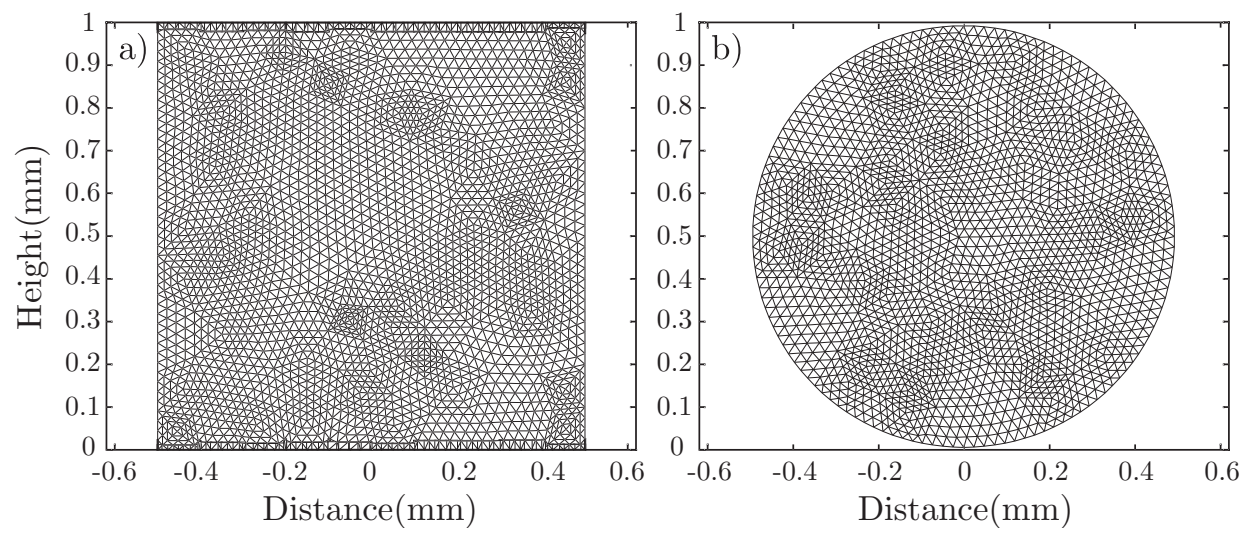

Figure 3.10. Pdetools meshing for a) rectangular coordinates and b) cylindrical coordinates.

of the cylindrical model the design consists of an infinitesimal cylinder represented by a circle which radius is equal to the sphere radius dimension of the implemented FDTD thermal model (Figure 3.10b)).

In this case the rectangle width is $1 \mathrm{~mm}$, because $0.5 \mathrm{~mm}$ radius drops are considered in the case of the FDTD model which should be validated. The height should be small to be considered infinitesimal, and $1 \mathrm{~mm}$ is also selected as an example. The radius in the circle of the cylindrical case is also considered $0.5 \mathrm{~mm}$.

The boundary conditions type Neumann are selected because of the air being considered to surround the material. They are defined for a surface temperature of $T_{S}=20^{\circ} \mathrm{C}$ and a convection heat transfer coefficient of $h_{T}=250 \mathrm{~W} \cdot \mathrm{m}^{-2} \cdot \mathrm{K}^{-1}$ (value chosen for forced convection in liquids [54]). In the rectangular model the boundary conditions are only defined on the sides of heating direction, while in the cylindrical case the $\mathrm{BC}$ are defined over the whole circumference. To specify the values in the pdetools, two variables are presented: $q=h_{T}$ and $g=h_{T} T_{S}$.

As seen in Figure 3.10, a mesh is applied at the surface to calculate the parabolic equation define in Equation 3.11. The selected thermal parameters, as in previous examples, are: $\rho=1000 \mathrm{~kg} \cdot \mathrm{m}^{-3}, c_{p}=4190 \mathrm{~J} \cdot \mathrm{kg}^{-1} \cdot \mathrm{K}^{-1}$, $k_{T}=0.6 \mathrm{~W} \cdot \mathrm{m}^{-1} \cdot \mathrm{K}^{-1}, Q_{g e n}=10^{9} \mathrm{~W} \cdot \mathrm{m}^{-3}$. The time process is selected to determine the convergence temperature values to a maximum. Hundred 

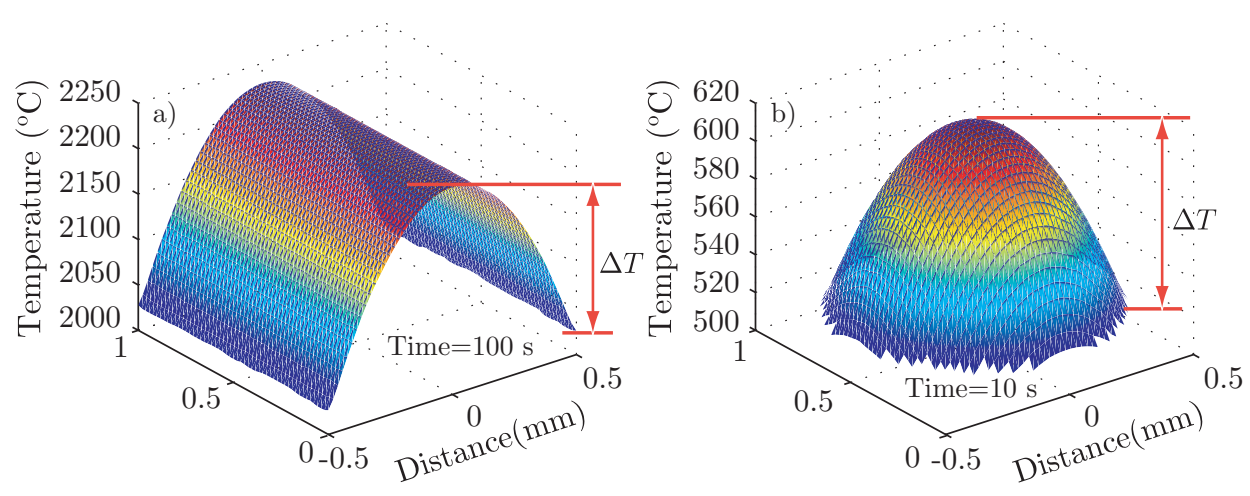

Figure 3.11. Temperature diffusion for the a) rectangular case and b) cylindrical case in Pdetools.

seconds are used in the rectangular case while only 10 seconds are used for the cylindrical one. Figure 3.11 shows the results for both cases.

The difference between the temperature at the central point (maximal temperature of convergence) and the temperature at the borders gives the maximal temperature deviation within the sample $(\Delta T)$ for each case. The values for the rectangular and cylindrical cases are respectively $208.399 \mathrm{~K}$ and $102.669 \mathrm{~K}$.

\subsubsection{Validation from a Literature Example}

Using the work reported by Incropera et al. [54], several simulations were carried out in order to corroborate the behaviour of the model. Figure 3.12 shows the evolution of the temperature profile with time for a specific example in [54], where a $20 \mathrm{~mm}$ fuel element of a nuclear reactor is convectively cooled at the surface with a convection heat transfer coefficient of $h=1100 \mathrm{~W} \cdot \mathrm{m}^{-2} \cdot \mathrm{K}^{-1}$ and $T_{\infty}=250^{\circ} \mathrm{C}$. This $U O_{2}$ fuel element, with thermal properties $k=30 \mathrm{~W} \cdot \mathrm{m}^{-1} \cdot \mathrm{K}^{-1}$ and $\alpha=5 \times 10^{-6} \mathrm{~m}^{2} \cdot \mathrm{s}^{-1}\left(\alpha=k_{T} / \rho \cdot c_{p}\right)$, is heated with $Q_{g_{e n}}=10^{7} \mathrm{~W} \cdot \mathrm{m}^{-3}$ at normal operating power. For the experiment the generation rate is changed to $Q_{g e n_{2}}=2 \times 10^{7} \mathrm{~W} \cdot \mathrm{m}^{-3}$. The effect of the convective cooling on the boundary points can be seen. After $150 \mathrm{~s}$ the temperature profile remains stable. 


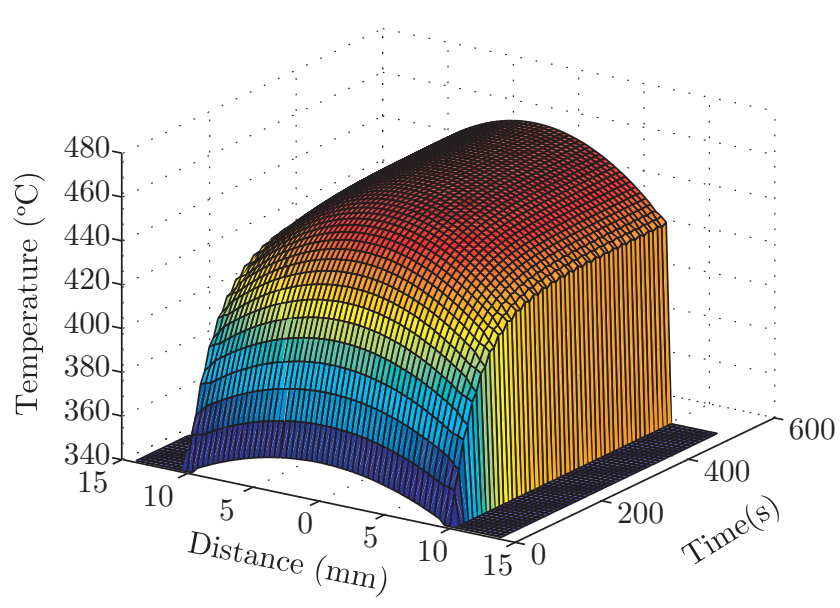

Figure 3.12. Spatial temperature distribution changing with time within a fuel element.

\subsubsection{Validation Results}

Table 3.3 summarizes the results of the FDTD model applied to the example reported in Section 3.5.2 and both validations (physically and pdetools validations) which are executed with the same parameters.

\begin{tabular}{lccc} 
& $\begin{array}{c}\text { Thermal } \\
\text { FDTD model }\end{array}$ & $\begin{array}{c}\text { Physical } \\
\text { validation }\end{array}$ & $\begin{array}{c}\text { Pdetool } \\
\text { validation }\end{array}$ \\
\hline Rectangular model & $208.241 \mathrm{~K}$ & $208.333 \mathrm{~K}$ & $208.399 \mathrm{~K}$ \\
Cylindrical model & $105.440 \mathrm{~K}$ & $104.166 \mathrm{~K}$ & $102.669 \mathrm{~K}$ \\
Spherical model & $70.228 \mathrm{~K}$ & $69.440 \mathrm{~K}$ & - \\
\hline
\end{tabular}

Table 3.3. Maximal temperature deviation values within the sample $(\Delta T)$. 


\begin{tabular}{lcc|cc}
$\begin{array}{l}\text { Time } \\
(\mathrm{s})\end{array}$ & $\begin{array}{c}\text { Temperature }\left({ }^{\circ} \mathrm{C}\right) \\
\text { Reference }[54]\end{array}$ & $\begin{array}{c}\text { at Boundary } \\
\text { Model }\end{array}$ & $\begin{array}{c}\text { Temperature }\left({ }^{\circ} \mathrm{C}\right) \\
\text { Reference }[54]\end{array}$ & $\begin{array}{c}\text { at Center } \\
\text { Model }\end{array}$ \\
\hline 0 & 340.91 & 340.91 & 357.58 & 357.57 \\
0.3 & 341.41 & 341.07 & 358.08 & 358.07 \\
0.6 & 341.88 & 341.64 & 358.58 & 358.56 \\
0.9 & 342.35 & 342.15 & 359.08 & 359.07 \\
1.2 & 342.82 & 342.63 & 359.58 & 359.56 \\
1.5 & 343.27 & 343.10 & 360.08 & 360.06 \\
$\infty$ & 431.82 & 432.78 & 465.15 & 465.14 \\
\hline
\end{tabular}

Table 3.4. Validation with an example from [54]. Temperature values are at the boundary and in the center of the sample.

A maximal error of $\pm 0.027 \%$ leads to the assumption of a validated model.

Table 3.4 shows the results up to 1.5 s extracted from the Figure 3.12 and compares these with the results of the example in [54].

The difference between the reference values [54] and the values calculated with the model is higher at the boundaries (maximum difference is $0.22 \%$ ) than in the center, where it is negligible (maximum difference is about $0.006 \%$ ). This high accordance can also be considered as a good validation of the model.

\subsection{Conclusions}

This chapter presented the performance of the thermal model when it is applied for the microwave heating of a material. The model was implemented with the FDTD technique in three coordinates systems: rectangular, cylindrical and spherical. Boundary conditions were applied to adjust the model to real conditions. The thermal variables used in the method were evaluated for a deeper knowledge of the model behaviour.

Several validations were applied to the thermal model for its evaluation. The results of all them confirmed the consistence of the model.

The main contributions of this chapter were published in [55]. 



\section{Microwave Cavities}

4.1 Introduction . . . . . . . . . . . . . . 71

4.2 Rectangular Cavities . . . . . . . . . . . . . . 72

4.3 Cylindrical Cavities . . . . . . . . . . . . . . 83

4.4 Cavity coupling . . . . . . . . . . . . . . . 88

4.5 Modelling of Microwave Cavities . . . . . . . . . . . 91

4.6 Perturbation Analysis of Cavities . . . . . . . . . . 103

4.7 Conclusions . . . . . . . . . . . . . . . . . . 118 



\section{Microwave Cavities}

THIS CHAPTER contains an introduction to the microwave rectangular and cylindrical cavities and their relation (characteristics and uses) to the PINE project. The electromagnetic fields, the resonant frequencies and quality factors expressions are given for several resonant modes in both cases: the case of empty cavities and the case of cavities loaded with dielectrics. The software used for the design of the cavities in this project is presented. Furthermore, the different coupling methods and the power transfer mechanisms of the cavities are explained using the perturbation method to analyse the losses when a dielectric sample is placed inside a cavity. Finally the thermal model from Chapter 3 is updated with the heat generation rate provided with the developed power transfer model.

\subsection{Introduction}

Transmission lines, e.g. waveguides, are usually employed in radio-frequency systems. Resonant cavities are produced by limiting the waveguides by means of two conducting plates positioned at the desired ends. The cavities are simply manufactured and used and they provide very high quality factors ( $Q$-factors), usually on the order of $5000-10000$, which make them 

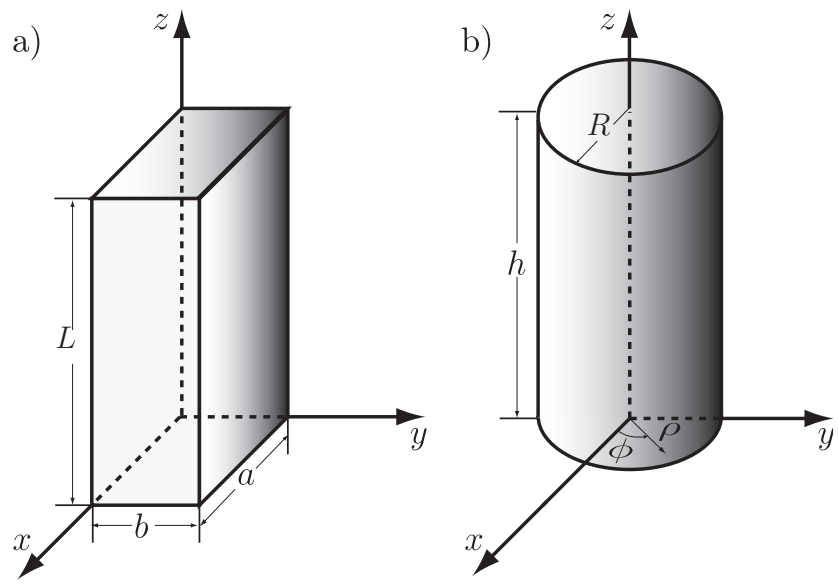

Figure 4.1. Geometry for the rectangular a) and cylindrical b) cavities.

attractive for measuring dielectric properties and/or heating materials.

The most common geometries of cavities are rectangular and cylindrical. The objective in this chapter is to introduce and analyse the characteristics and parameters of the cavities applied in the context of this manuscript.

Rectangular cavities, of which the geometry is represented in Figure $4.1 \mathrm{a}$ ), are applied to trigger the internal gelation of the feed solution in form of drops and obtain the SP fuel. Cylindrical cavities, represented in Figure $4.1 \mathrm{~b}$ ), are principally used to measure the dielectric properties of the feed solution (Chapter 5).

\subsection{Rectangular Cavities}

A cavity is a waveguide closed by two perfect matching conductors, where its electromagnetic solution consists at the superposition of an incident and a reflected waves $[56,57]$. If the fields are separated in tangential and axial components and the propagation is along the $\mathrm{z}$ axis, this means for the 
Transversal Electric (TE) modes

$$
\begin{aligned}
\vec{E} & =\vec{E}_{0 t}(x, y)\left(e^{-j \beta_{z}}-\rho e^{j \beta_{z}}\right) \\
\vec{H} & =\vec{H}_{0 t}(x, y)\left(e^{-j \beta_{z}}+\rho e^{j \beta_{z}}\right)+\vec{H}_{0 z}(x, y)\left(e^{-j \beta_{z}}-\rho e^{j \beta_{z}}\right)
\end{aligned}
$$

where $\beta_{z}$ is the phase constant in $z$ direction $\left(\mathrm{rad} \cdot \mathrm{m}^{-1}\right)$ and $\rho$ is the reflection factor. In case of the Transversal Magnetic (TM) modes the fields are given by

$$
\begin{aligned}
\vec{E} & =\vec{E}_{0 t}(x, y)\left(e^{-j \beta_{z}}-\rho e^{j \beta_{z}}\right)+\vec{E}_{0 z}(x, y)\left(e^{-j \beta_{z}}+\rho e^{j \beta_{z}}\right) \\
\vec{H} & =\vec{H}_{0 t}(x, y)\left(e^{-j \beta_{z}}+\rho e^{j \beta_{z}}\right)
\end{aligned}
$$

Because of the rectilinear geometry (rectangular waveguide of section $a \times b$ ) the propagation equations are defined using a rectangular coordinate system. For the TE modes the equations are given by

$$
\begin{aligned}
E_{x} & =Z_{T E} H_{y}=A_{m n} \frac{j w \mu_{0}}{k_{c, m n}^{2}} \beta_{y} \cos \left(\beta_{x} x\right) \sin \left(\beta_{y} y\right) e^{-\gamma_{z} z} \\
E_{y} & =-Z_{T E} H_{x}=-A_{m n} \frac{j w \mu_{0}}{k_{c, m n}^{2}} \beta_{x} \sin \left(\beta_{x} x\right) \cos \left(\beta_{y} y\right) e^{-\gamma_{z} z} \\
E_{z} & =0 \\
H_{x} & =A_{m n} \frac{\gamma_{z}}{k_{c, m n}^{2}} \beta_{x} \sin \left(\beta_{x} x\right) \cos \left(\beta_{y} y\right) e^{-\gamma_{z} z} \\
H_{y} & =A_{m n} \frac{\gamma_{z}}{k_{c, m n}^{2}} \beta_{y} \cos \left(\beta_{x} x\right) \sin \left(\beta_{y} y\right) e^{-\gamma_{z} z} \\
H_{z} & =A_{m n} \cos \left(\beta_{x} x\right) \cos \left(\beta_{y} y\right) e^{-\gamma_{z} z}
\end{aligned}
$$

where $A_{m n}$ is a constant dependent on the incident power and the mode wave numbers or mode phase constants in the $x$ and $y$ direction are

$$
\left.\begin{array}{ll}
\beta_{x}=\frac{m \pi}{a} & m=0,1,2, \ldots \\
\beta_{y}=\frac{n \pi}{b} & n=0,1,2, \ldots
\end{array}\right\} m=n \neq 0
$$


In the case of the TM modes the propagation equations are

$$
\begin{aligned}
E_{x} & =-B_{m n} \frac{\gamma_{z}}{k_{c, m n}^{2}} \beta_{x} \cos \left(\beta_{x} x\right) \sin \left(\beta_{y} y\right) e^{-\gamma_{z} z} \\
E_{y} & =-B_{m n} \frac{\gamma_{z}}{k_{c, m n}^{2}} \beta_{y} \sin \left(\beta_{x} x\right) \cos \left(\beta_{y} y\right) e^{-\gamma_{z} z} \\
E_{z} & =B_{m n} \sin \left(\beta_{x} x\right) \sin \left(\beta_{y} y\right) e^{-\gamma_{z} z} \\
H_{x} & =-\frac{E_{y}}{Z_{T M}} \\
& =B_{m n} \frac{j w \varepsilon_{0} \varepsilon_{r}(1-j \tan \delta)}{k_{c, m n}^{2}} \beta_{y} \sin \left(\beta_{x} x\right) \cos \left(\beta_{y} y\right) e^{-\gamma_{z} z} \\
H_{y} & =\frac{E_{x}}{Z_{T M}} \\
& =-B_{m n} \frac{j w \varepsilon_{0} \varepsilon_{r}(1-j \tan \delta)}{k_{c, m n}^{2}} \beta_{x} \cos \left(\beta_{x} x\right) \sin \left(\beta_{y} y\right) e^{-\gamma_{z} z} \\
H_{z} & =0
\end{aligned}
$$

where $B_{m n}$ is a constant dependent on the incident power and the phase constants are defined by

$$
\left.\begin{array}{ll}
\beta_{x}=\frac{m \pi}{a} & m=1,2,3, \ldots \\
\beta_{y}=\frac{n \pi}{b} & n=1,2,3, \ldots
\end{array}\right\}
$$

For both TE and TM modes, the characteristic relations of the guide are given by the following equations

$$
\begin{aligned}
& k_{c, m n}=\sqrt{\beta_{x}^{2}+\beta_{y}^{2}} \\
& k^{2}=k_{c, m n}^{2}-\gamma_{z}^{2} \\
& k^{2}=w^{2} \mu_{0} \varepsilon_{0} \varepsilon_{r}(1-j \tan \delta) \\
& \gamma_{z}=\sqrt{k_{c, m n}^{2}-k^{2}}=\alpha_{z}+j \beta_{z} \\
& \tan \delta=\frac{\varepsilon^{\prime \prime}}{\varepsilon^{\prime}} \\
& Z_{T M}=\frac{\gamma_{z}}{j \omega \varepsilon_{0} \varepsilon_{r}(1-j \tan \delta)}=\frac{\gamma_{z}}{j k} \eta^{\prime}
\end{aligned}
$$




$$
\begin{aligned}
& Z_{T E}=\frac{j \omega \mu_{0}}{\gamma_{z}}=\frac{j k}{\gamma_{z}} \eta^{\prime} \\
& \eta=\sqrt{\frac{\mu_{0}}{\varepsilon_{0} \varepsilon_{r}(1-j \tan \delta)}}
\end{aligned}
$$

where $k_{c, m n}$ is the cutoff wave number and $k$ refers to the propagation constant in free space. $\gamma_{z}$ is the propagation constant with $\alpha_{z}$ the attenuation constant and $\beta_{z}$ the phase constant of the TE or TM mode. $\varepsilon_{0}$ and $\mu_{0}$ are the vacuum permittivity and permeability respectively, $\varepsilon_{r}=\varepsilon^{\prime}$ is the relative dielectric constant and $\tan \delta$ is the dielectric loss tangent, both defined by the complex relative permittivity of a homogeneous dielectric $\varepsilon^{*}=\varepsilon^{\prime}-j \varepsilon^{\prime \prime} . Z_{T M}$ and $Z_{T E}$ represent the characteristic impedance of the $\mathrm{TM}$ and TE guided modes respectively, and $\eta^{\prime}$ represents the characteristic impedance for a Transversal Electromagnetic (TEM) wave in free space.

Developing the expressions 4.1 and 4.2 in function of the progressive and regressive waves and applying the boundary conditions, i.e. the perfectly conducting end plates in $z=0$ and $z=L$ to define the rectangular cavity, the solutions of the electromagnetic fields inside the cavity are expressed for the $\mathrm{TE}_{m n p}$ modes as

$$
\begin{aligned}
E_{x} & =A_{m n} \frac{w \mu_{0}}{k_{c, m n}^{2}} \beta_{y} \cos \left(\beta_{x} x\right) \sin \left(\beta_{y} y\right) \sin \left(\beta_{z} z\right) \\
E_{y} & =-A_{m n} \frac{w \mu_{0}}{k_{c, m n}^{2}} \beta_{x} \sin \left(\beta_{x} x\right) \cos \left(\beta_{y} y\right) \sin \left(\beta_{z} z\right) \\
E_{z} & =0 \\
H_{x} & =A_{m n} \frac{j \beta_{z}}{k_{c, m n}^{2}} \beta_{x} \sin \left(\beta_{x} x\right) \cos \left(\beta_{y} y\right) \cos \left(\beta_{z} z\right) \\
H_{y} & =A_{m n} \frac{j \beta_{z}}{k_{c, m n}^{2}} \beta_{y} \cos \left(\beta_{x} x\right) \sin \left(\beta_{y} y\right) \cos \left(\beta_{z} z\right) \\
H_{z} & =-j A_{m n} \cos \left(\beta_{x} x\right) \cos \left(\beta_{y} y\right) \sin \left(\beta_{z} z\right)
\end{aligned}
$$

where the mode phase constants in the $x, y$ and $z$ direction are

$$
\left.\begin{array}{ll}
\beta_{x}=\frac{m \pi}{a} & m=0,1,2, \ldots \\
\beta_{y}=\frac{n \pi}{b} & n=0,1,2, \ldots \\
\beta_{z}=\frac{p \pi}{L} & p=1,2,3, \ldots
\end{array}\right\} m=n \neq 0
$$


In the case of the $\mathrm{TM}_{m n p}$ modes:

$$
\begin{aligned}
E_{x} & =-B_{m n} \frac{\beta_{z}}{k_{c, m n}^{2}} \beta_{x} \cos \left(\beta_{x} x\right) \sin \left(\beta_{y} y\right) \sin \left(\beta_{z} z\right) \\
E_{y} & =-B_{m n} \frac{\beta_{z}}{k_{c, m n}^{2}} \beta_{y} \sin \left(\beta_{x} x\right) \cos \left(\beta_{y} y\right) \sin \left(\beta_{z} z\right) \\
E_{z} & =B_{m n} \sin \left(\beta_{x} x\right) \sin \left(\beta_{y} y\right) \cos \left(\beta_{z} z\right) \\
H_{x} & =B_{m n} \frac{j w \varepsilon_{0}}{k_{c, m n}^{2}} \beta_{y} \sin \left(\beta_{x} x\right) \cos \left(\beta_{y} y\right) \cos \left(\beta_{z} z\right) \\
H_{y} & =-B_{m n} \frac{j w \varepsilon_{0}}{k_{c, m n}^{2}} \beta_{x} \cos \left(\beta_{x} x\right) \sin \left(\beta_{y} y\right) \cos \left(\beta_{z} z\right) \\
H_{z} & =0
\end{aligned}
$$

where the mode phase constants in the $x, y$ and $z$ direction are

$$
\left.\begin{array}{ll}
\beta_{x}=\frac{m \pi}{a} & m=1,2,3, \ldots \\
\beta_{y}=\frac{n \pi}{b} & n=1,2,3, \ldots \\
\beta_{z}=\frac{p \pi}{L} & p=0,1,2 \ldots
\end{array}\right\}
$$

Similarly to the case of the waveguide equations, $A_{m n}$ and $B_{m n}$ are constants related with the incident power. $a, b$ and $L$ are respectively the width, height and length of the cavity. $\varepsilon_{0}$ and $\mu_{0}$ are respectively the vacuum permittivity and permeability, and $k_{0}$ is the free space propagation constant, which is given by the following equations in the case of an empty cavity

$$
\begin{aligned}
& k_{0}^{2}=k_{c, m n}^{2}+\beta_{z}^{2} \\
& k_{0}^{2}=\omega_{u 0}^{2} \mu_{0} \varepsilon_{0} \\
& \omega_{u 0}=\frac{1}{\sqrt{\mu_{0} \varepsilon_{0}}} \sqrt{\left(\frac{m \pi}{a}\right)^{2}+\left(\frac{n \pi}{b}\right)^{2}+\left(\frac{p \pi}{L}\right)^{2}}
\end{aligned}
$$

where $\omega_{u 0}$ is the angular resonant frequency of an empty or unloaded cavity.

In the case of a cavity filled with a dielectric, the equations include the 
term of the lossy dielectric through the relative permittivity $\varepsilon_{r}$ as

$$
\begin{aligned}
& k_{0}^{2}=k_{c, m n}^{2}+\beta_{z}^{2} \\
& k_{0}^{2}=w^{2} \mu_{0} \varepsilon_{0} \varepsilon_{r}(1-j \tan \delta) \\
& \tan \delta=\frac{\varepsilon^{\prime \prime}}{\varepsilon^{\prime}} \\
& \omega_{u}=\frac{1}{\sqrt{\mu_{0} \varepsilon_{0} \varepsilon_{r}}} \sqrt{\left(\frac{m \pi}{a}\right)^{2}+\left(\frac{n \pi}{b}\right)^{2}+\left(\frac{p \pi}{L}\right)^{2}}=\frac{\omega_{u 0}}{\sqrt{\varepsilon_{r}}}
\end{aligned}
$$

where $w_{u}$ is the angular unloaded resonant frequency of the cavity.

The solution of the electromagnetic fields is the same for both the case of an empty cavity and the case of the cavity filled with a dielectric. The resonance in stable regime only exists in the case of a lossless medium $(\tan \delta=0)$. In lossy media the fields will vanish with time but the losses can be compensated by applying energy to the cavity.

\subsubsection{Empty Rectangular Cavities}

The quality factor ( $Q$-factor) of an empty cavity, $Q_{u 0}$, is related to the losses associated to this cavity. Considering the perturbation method [56, 57], the fields in a cavity with small losses and the fields of a lossless cavity, i.e. cavity with perfect conductive walls, are assumed to be the same. However, the walls are lossy surfaces, the losses of which are represented by a surface impedance $Z_{s}[56]$ :

$$
Z_{s}=R_{s}+j X_{s}=\sqrt{\frac{j \omega \mu_{0}}{\sigma+j \omega \varepsilon_{0}}} \stackrel{\sigma \gg \omega \varepsilon_{0}}{\cong} \sqrt{\frac{j \omega \mu_{0}}{\sigma}}=\sqrt{\frac{\omega \mu_{0}}{2 \sigma}}(1+j)
$$

where $\sigma$ is the metal conductivity $\left(\mathrm{S} \cdot \mathrm{m}^{-1}\right), R_{s}$ and $X_{s}$ are the surface resistance and reactance of a conductor respectively $(\Omega)$.

From this concept, the $Q$-factor is defined by the relation between the stored energy and the dissipated power of the cavity.

$$
Q_{u 0}=Q_{c 0} \equiv \omega \frac{\text { stored energy }}{\text { dissipated power }}=\left.\omega \frac{W_{e}+W_{m}}{P_{0}}\right|_{\omega=\omega_{u 0}}
$$

where $\omega_{u 0}=2 \pi f_{u 0}$ is the angular unloaded resonant frequency of an empty cavity, linked to the frequency, which is calculated from its relation with 
the free space propagation constant as:

$$
f_{u 0, m n p}=\frac{\omega_{u 0}}{2 \pi}=\frac{1}{2 \pi \sqrt{\mu_{0} \varepsilon_{0}}} \sqrt{\left(\frac{m \pi}{a}\right)^{2}+\left(\frac{n \pi}{b}\right)^{2}+\left(\frac{p \pi}{L}\right)^{2}}
$$

The stored electric $W_{e}$ and magnetic $W_{m}$ energies are proportional to the cavity volume $V_{c}\left(\mathrm{~m}^{3}\right)$ :

$$
\begin{aligned}
& W_{e}=\frac{\varepsilon_{0}}{4} \int_{V_{c}} \vec{E} \cdot \vec{E}^{*} d V=\frac{\varepsilon_{0}}{4} \int_{V_{c}}|\vec{E}|^{2} d v \\
& W_{m}=\frac{\mu_{0}}{4} \int_{V_{c}} \vec{H} \cdot \vec{H}^{*} d V=\frac{\mu_{0}}{4} \int_{V_{c}}|\vec{H}|^{2} d v
\end{aligned}
$$

where $\vec{E}$ is the electric field inside the cavity and $\vec{H}$ is the magnetic field inside the cavity.

Nevertheless, the real dissipated power in the walls of the cavity is proportional to its surface $S_{c}\left(\mathrm{~m}^{2}\right)$ :

$$
P_{0}=\frac{R_{s}}{2} \int_{S_{c}}\left|\vec{H}_{\tan }\right|^{2} d S
$$

where $\left|\vec{H}_{\text {tan }}\right|$ is the magnetic field tangent to the cavity walls and $R_{s}$ is the surface resistance of the walls, which for a metal with small losses is calculated as

$$
R_{s}=\sqrt{\frac{\omega_{u 0} \mu_{0}}{2 \sigma}}
$$

\section{Calculation of energies and powers related to the empty rectangular cavity}

Applying the electromagnetic field Equations 4.13 and 4.14, the average stored electric and magnetic energies equations are expressed as follows (development in Appendix C).

For the TE modes (with $m=0,1,2, \ldots, n=0,1,2, \ldots(m=n \neq 0)$ and $p=1,2,3, \ldots)$ :

$$
\begin{aligned}
& W_{e}=\frac{\varepsilon_{0}}{4} \frac{A_{m n}^{2} \omega^{2} \mu_{0}^{2}}{k_{c, m n}^{2}} \frac{a b L}{8} \epsilon_{m} \epsilon_{n} \\
& W_{m}=\frac{\mu_{0}}{4} A_{m n}^{2} \frac{a b L}{8} \epsilon_{m} \epsilon_{n}\left(1+\frac{\beta_{z}^{2}}{k_{c, m n}^{2}}\right)
\end{aligned}
$$


where:

$$
\begin{aligned}
\epsilon_{m} & = \begin{cases}2, & m=0 \\
1, & m \neq 0\end{cases} \\
\epsilon_{n} & = \begin{cases}2, & n=0 \\
1, & n \neq 0\end{cases}
\end{aligned}
$$

For the TM modes (with $m=1,2,3, \ldots, n=1,2,3, \ldots$ and $p=$ $0,1,2, \ldots)$ :

$$
\begin{aligned}
& W_{e}=\frac{\varepsilon_{0}}{4} B_{m n}^{2} \frac{a b L}{8} \epsilon_{p}\left(1+\frac{\beta_{z}^{2}}{k_{c, m n}^{2}}\right) \\
& W_{m}=\frac{\mu_{0}}{4} \frac{B_{m n}^{2} \omega_{u}^{2} \varepsilon_{0}^{2}}{k_{c, m n}^{2}} \frac{a b L}{8} \epsilon_{p}
\end{aligned}
$$

where:

$$
\epsilon_{p}= \begin{cases}2, & p=0 \\ 1, & p \neq 0\end{cases}
$$

The real dissipated power by the walls of the cavity for the $\mathrm{TE}_{m n p}$ modes (with $m=0,1,2, \ldots, n=0,1,2, \ldots(m=n \neq 0)$ and $p=1,2,3, \ldots)$ is

$$
P_{0}=R_{s} A_{m n}^{2}\left\{\frac{\beta_{z}^{2}}{k_{c, m n}^{4}}\left[\frac{a b}{4} \epsilon_{m} \epsilon_{n} k_{c, m n}^{2}+\frac{L}{4}\left(\frac{(m \pi)^{2}}{a}+\frac{(n \pi)^{2}}{b}\right)\right]+\frac{L}{4}\left(a \epsilon_{m}+b \epsilon_{n}\right)\right\}
$$

and for the $\mathrm{TM}_{m n p}$ modes (with $m=1,2,3, \ldots, n=1,2,3, \ldots$ and $p=$ $0,1,2, \ldots)$ is

$$
P_{0}=R_{s} \frac{B_{m n}^{2} \omega^{2} \varepsilon_{0}^{2}}{k_{c, m n}^{4}}\left\{\frac{a b}{4} k_{c, m n}^{2}+\frac{L}{4} \epsilon_{p}\left[b\left(\frac{m \pi}{a}\right)^{2}+a\left(\frac{n \pi}{b}\right)^{2}\right]\right\}
$$

\subsubsection{Rectangular Cavities filled with homogeneous dielectrics}

The $Q$-factor of a cavity completely filled with a homogeneous dielectric is dictated by the losses of the dielectric:

$$
Q_{d}=\frac{1}{\tan \delta}
$$


which is related to the complex resonance frequency (further explanation presented later in Section 4.6.2) as:

$$
\Omega=\omega_{u}\left(1+\frac{j}{2 Q_{d}}\right)
$$

From this equation the resonant frequency of the cavity is obtained:

$$
f_{u}=\frac{1}{2 \sqrt{\mu_{0} \varepsilon_{0} \varepsilon_{r}}} \sqrt{\left(\frac{m}{a}\right)^{2}+\left(\frac{n}{b}\right)^{2}+\left(\frac{p}{L}\right)^{2}}
$$

In the case of no losses on the walls of the cavity, the $Q$-factor is defined like in the case of the empty cavity (Equation 4.18) but referring to the dissipated power in the dielectric:

$$
Q_{d}=\omega \frac{W_{e}+W_{m}}{P_{d}}
$$

The stored magnetic energy is calculated with the same expression than the empty cavity (Equation 4.21) and the stored electric energy, which includes the relative dielectric constant, is read as:

$$
W_{e}=\frac{\varepsilon_{0} \varepsilon_{r}}{4} \int_{V_{c}} \vec{E} \cdot \vec{E}^{*} d V=\frac{\varepsilon_{0} \varepsilon_{r}}{4} \int_{V_{c}}|\vec{E}|^{2} d v
$$

which is also dependent on the cavity volume.

The dissipated power in the sample is proportional to its volume $\left(v_{s}\right)$.

$$
P_{d}=\frac{\sigma_{e}}{2} \int_{v_{s}}|\vec{E}|^{2} d v
$$

where $\sigma_{e}$ is the equivalent dielectric conductivity given by:

$$
\sigma_{e}=\omega \varepsilon_{0} \varepsilon^{\prime \prime}=\omega \varepsilon_{0} \varepsilon_{r} \tan \delta
$$

In case of lossy walls the $Q$-factor of the cavity filled with the dielectric is calculated as

$$
Q_{c}=\left.\omega \frac{W_{e}+W_{m}}{P_{0}}\right|_{\omega=\omega_{u}}=\omega_{u} \frac{2 W_{e}}{P_{0}}=\omega_{u} \frac{2 W_{m}}{P_{0}}
$$


being the same expression than for the $Q$-factor of the empty cavity $\left(Q_{u 0}\right)$ but calculated for the frequency of the perturbed cavity $w_{u} . P_{0}$ is calculated with Equation 4.22 also including $w_{u}$ in the calculation of $R_{s}$.

The total $Q$-factor of the cavity is defined as

$$
Q_{u}=\left.\omega \frac{W_{e}+W_{m}}{P_{t}}\right|_{\omega=\omega_{u}}
$$

where $P_{t}=P_{0}+P_{d}$ is the real dissipated power in the cavity, i.e. the sum of the dissipated power on the walls of the cavity and the dissipated power in the dielectric. The relation between $Q$-factors is then

$$
\frac{1}{Q_{u}}=\frac{1}{Q_{c}}+\frac{1}{Q_{d}}
$$

Calculation of energies and powers in the cavity completely filled with a dielectric

Applying the electromagnetic field Equations 4.13 and 4.14, and following the same procedure than for the empty cavity, the average stored electric and magnetic energy equations are expressed as follows

For the TE modes (with $m=0,1,2, \ldots, n=0,1,2, \ldots(m=n \neq 0)$ and $p=1,2,3, \ldots)$ :

$$
\begin{aligned}
& W_{e}=\frac{\varepsilon_{0} \varepsilon_{r}}{4} \frac{A_{m n}^{2} \omega_{u}^{2} \mu_{0}^{2}}{k_{c, m n}^{2}} \frac{a b L}{8} \epsilon_{m} \epsilon_{n} \\
& W_{m}=\frac{\mu_{0}}{4} A_{m n}^{2} \frac{a b L}{8} \epsilon_{m} \epsilon_{n}\left(1+\frac{\beta_{z}^{2}}{k_{c, m n}^{2}}\right)
\end{aligned}
$$

where:

$$
\begin{aligned}
\epsilon_{m} & = \begin{cases}2, & m=0 \\
1, & m \neq 0\end{cases} \\
\epsilon_{n} & = \begin{cases}2, & n=0 \\
1, & n \neq 0\end{cases}
\end{aligned}
$$

For the TM modes (with $m=1,2,3, \ldots, n=1,2,3, \ldots$ and $p=$ 
$0,1,2, \ldots):$

$$
\begin{aligned}
& W_{e}=\frac{\varepsilon_{0} \varepsilon_{r}}{4} B_{m n}^{2} \frac{a b L}{8} \epsilon_{p}\left(1+\frac{\beta_{z}^{2}}{k_{c, m n}^{2}}\right) \\
& W_{m}=\frac{\mu_{0}}{4} \frac{B_{m n}^{2} \omega_{u}^{2} \varepsilon_{0}^{2} \varepsilon_{r}^{2}}{k_{c, m n}^{2}} \frac{a b L}{8} \epsilon_{p}
\end{aligned}
$$

where:

$$
\epsilon_{p}= \begin{cases}2, & p=0 \\ 1, & p \neq 0\end{cases}
$$

In case the dielectric occupies the whole cavity volume and there are lossless walls, the real power absorbed by the dielectric for the $\mathrm{TE}_{m n p}$ modes (with $m=0,1,2, \ldots, n=0,1,2, \ldots(m=n \neq 0)$ and $p=1,2,3, \ldots)$ is

$$
P_{d}=\frac{\omega_{u} \varepsilon_{0} \varepsilon_{r} \tan \delta}{2} \frac{A_{m n}^{2} \omega_{u}^{2} \mu_{0}^{2}}{k_{c, m n}^{2}} \frac{a b L}{8} \epsilon_{m} \epsilon_{n}
$$

and for the $\mathrm{TM}_{m n p}$ modes (with $m=1,2,3, \ldots, n=1,2,3, \ldots$ and $p=$ $0,1,2, \ldots)$ is

$$
P_{d}=\frac{\omega_{u} \varepsilon_{0} \varepsilon_{r} \tan \delta}{2} B_{m n}^{2} \frac{a b L}{8} \epsilon_{p}\left(1+\frac{\beta_{z}^{2}}{k_{c, m n}^{2}}\right)
$$

In the case of lossy walls, for the TE and TM modes the dissipated power on the walls of the cavity $P_{0}$ reads respectively

$$
\begin{gathered}
P_{0}=R_{s} A_{m n}^{2}\left\{\frac{\beta_{z}^{2}}{k_{c, m n}^{4}}\left[\frac{a b}{4} \epsilon_{m} \epsilon_{n} k_{c, m n}^{2}+\frac{L}{4}\left(\frac{(m \pi)^{2}}{a}+\frac{(n \pi)^{2}}{b}\right)\right]+\frac{L}{4}\left(a \epsilon_{m}+b \epsilon_{n}\right)\right\} \\
P_{0}=R_{s} \frac{B_{m n}^{2} \omega_{u}^{2} \varepsilon_{0}^{2} \varepsilon_{r}^{2}}{k_{c, m n}^{4}}\left\{\frac{a b}{4} k_{c, m n}^{2}+\frac{L}{4} \epsilon_{p}\left[b\left(\frac{m \pi}{a}\right)^{2}+a\left(\frac{n \pi}{b}\right)^{2}\right]\right\}
\end{gathered}
$$




\subsection{Cylindrical Cavities}

The electromagnetic equations in a cylindrical cavity, as in the rectangular cavity, are calculated from the sum of an incident and a reflected waves in a $R$ radius cylindrical waveguide which is limited by two walls in $z=0$ and $z=h$ (Figure $4.1 \mathrm{~b})$ ). Considering perfectly conductive walls for the TE modes, these equations are expressed, in cylindrical coordinates because of the tubular geometry [57], as

$$
\begin{aligned}
E_{\rho} & =A_{m n} \frac{w \mu}{k_{c, m n}^{\prime 2}} \frac{m}{\rho} \sin (m \phi) J_{m}\left(k_{c, m n}^{\prime} \rho\right) \sin \left(\beta_{z} z\right) \\
E_{\phi} & =A_{m n} \frac{w \mu}{k_{c, m n}^{\prime 2}} \cos (m \phi) J_{m}^{\prime}\left(k_{c, m n}^{\prime} \rho\right) \sin \left(\beta_{z} z\right) \\
E_{z} & =0 \\
H_{\rho} & =-A_{m n} \frac{j \beta_{z}}{k_{c, m n}^{\prime}} \cos (m \phi) J_{m}^{\prime}\left(k_{c, m n}^{\prime} \rho\right) \cos \left(\beta_{z} z\right) \\
H_{\phi} & =A_{m n} \frac{j \beta_{z}}{k_{c, m n}^{\prime 2}} \frac{m}{\rho} \sin (m \phi) J_{m}\left(k_{c, m n}^{\prime} \rho\right) \cos \left(\beta_{z} z\right) \\
H_{z} & =-j A_{m n} \cos (m \phi) J_{m}\left(k_{c, m n}^{\prime} \rho\right) \sin \left(\beta_{z} z\right)
\end{aligned}
$$

(for $m=0,1,2, \ldots, n=1,2,3, \ldots$ and $p=1,2,3, \ldots$ ). The mode phase constant in $z$ direction is given by:

$$
\beta_{z}=\sqrt{k_{0}^{2}-k_{c, m n}^{\prime 2}}=\frac{p \pi}{h}
$$

then,

$$
k_{c, m n}^{\prime}=\frac{P_{m n}^{\prime}}{R}
$$

where $P_{m n}^{\prime}$ is the $n$th zero of the derivative $\left(J_{m}^{\prime}(x)\right)$ of the Bessel function of the first kind $J_{m}(x)$ (see Table D.2 in Appendix D), without considering the zero at the origin when $m=0$. 
In case of the TM modes, for $(m=0,1,2, \ldots, n=1,2,3, \ldots$ and $p=0,1,2, \ldots)$ :

$$
\begin{aligned}
E_{\rho} & =-B_{m n} \frac{\beta_{z}}{k_{c, m n}} \cos (m \phi) J_{m}^{\prime}\left(k_{c, m n} \rho\right) \sin \left(\beta_{z} z\right) \\
E_{\phi} & =B_{m n} \frac{\beta_{z}}{k_{c, m n}^{2}} \frac{m}{\rho} \sin (m \phi) J_{m}\left(k_{c, m n} \rho\right) \sin \left(\beta_{z} z\right) \\
E_{z} & =B_{m n} \cos (m \phi) J_{m}\left(k_{c, m n} \rho\right) \cos \left(\beta_{z} z\right) \\
H_{\rho} & =-B_{m n} \frac{j w \varepsilon_{0}}{k_{c, m n}^{2}} \frac{m}{\rho} \sin (m \phi) J_{m}\left(k_{c, m n} \rho\right) \cos \left(\beta_{z} z\right) \\
H_{\phi} & =-B_{m n} \frac{j w \varepsilon_{0}}{k_{c, m n}} \cos (m \phi) J_{m}^{\prime}\left(k_{c, m n} \rho\right) \cos \left(\beta_{z} z\right) \\
H_{z} & =0
\end{aligned}
$$

where the mode phase constant in $z$ direction is given by:

$$
\beta_{z}=\sqrt{k_{0}^{2}-k_{c, m n}^{2}}=\frac{p \pi}{h}
$$

then,

$$
k_{c, m n}=\frac{P_{m n}}{R}
$$

where $P_{m n}$ is the $n$th zero of the Bessel function of the first kind $J_{m}(x)$ (see Table D.1 in Appendix D), without considering the zero at the origin when $m \neq 0$.

$A_{m n}$ and $B_{m n}$ are constants related with the incident power. $R$ and $h$ are respectively the radius and height of the cavity. $\varepsilon_{0}$ and $\mu_{0}$ are respectively the vacuum permittivity and permeability, and $k_{0}$ is the free space propagation constant, which is given by the Equation 4.15 a) in the case of an empty cavity.

\subsubsection{Empty Cylindrical Cavities}

The resonant frequencies for the TE and TM modes in an empty cavity are given respectively as:

$$
f_{u 0, m n p}=\frac{1}{2 \pi \sqrt{\mu_{0} \varepsilon_{0}}} \sqrt{\left(\frac{P_{m n}^{\prime}}{R}\right)^{2}+\left(\frac{p \pi}{h}\right)^{2}}
$$




$$
f_{u 0, m n p}=\frac{1}{2 \pi \sqrt{\mu_{0} \varepsilon_{0}}} \sqrt{\left(\frac{P_{m n}}{R}\right)^{2}+\left(\frac{p \pi}{h}\right)^{2}}
$$

The quality factor $Q_{u 0}$ is calculated with the same expression than for the rectangular cavities 4.18, where the energies are calculated with Equations 4.20 and 4.21 and the dissipated power with Equation 4.22.

Applying the electromagnetic field equations in the cylindrical cavity (Equations 4.44 and 4.47, the average stored electric and magnetic energies for the TE modes with $(m=0,1,2, \ldots, n=1,2,3, \ldots$ and $p=1,2,3, \ldots)$ are:

$$
\begin{aligned}
& W_{e}=\frac{\varepsilon_{0}}{16} \pi \epsilon_{m} \frac{A_{m n}^{2} \omega^{2} \mu_{0}^{2} R^{4} h}{P_{m n}^{\prime 2}}\left(1-\frac{m^{2}}{P_{m n}^{\prime 2}}\right) J_{m}^{2}\left(P_{m n}^{\prime}\right) \\
& W_{m}=\frac{\mu_{0}}{16} \pi \epsilon_{m} \frac{A_{m n}^{2} R^{2} h}{P_{m n}^{\prime 2}}\left(1-\frac{m^{2}}{P_{m n}^{\prime 2}}\right) J_{m}^{2}\left(P_{m n}^{\prime}\right) \cdot\left[\beta_{z}^{2} R^{2}+P_{m n}^{\prime 2}\right]
\end{aligned}
$$

and the dissipated power is read as

$$
P_{0}=\frac{R_{s}}{2} \epsilon_{m} \pi A_{m n}^{2} \cdot J_{m}^{2}\left(P_{m n}^{\prime}\right)\left[\frac{R h}{2}\left(1+\frac{m^{2} \beta_{z}^{2} R^{2}}{P_{m n}^{\prime 4}}\right)+\frac{\beta_{z}^{2} R^{4}}{P_{m n}^{\prime 2}}\left(1-\frac{m^{2}}{P_{m n}^{\prime 2}}\right)\right]
$$

In case of TM modes with $(m=0,1,2, \ldots, n=1,2,3, \ldots$ and $p=$ $0,1,2, \ldots)$ the stored electric and magnetic energies are given as

$$
\begin{aligned}
& W_{e}=\frac{\varepsilon_{0}}{16} \pi h \epsilon_{m} \epsilon_{p} \frac{B_{m n}^{2} R^{2} J_{m}^{\prime 2}\left(P_{m n}\right)}{P_{m n}^{2}}\left[P_{m n}^{2}+\beta_{z}^{2} R^{2}\right] \\
& W_{m}=\frac{\mu_{0}}{16} \pi \epsilon_{m} \epsilon_{p} \frac{B_{m n}^{2} \omega^{2} \varepsilon_{0}^{2} R^{4} h}{P_{m n}^{2}} J_{m}^{\prime 2}\left(P_{m n}\right)
\end{aligned}
$$

and the dissipated power is

$$
P_{0}=\frac{R_{s}}{2} \epsilon_{m} \pi B_{m n}^{2} \omega^{2} \varepsilon_{0}^{2} \frac{1}{k_{c}^{2}} J_{m}^{\prime 2}\left(P_{m n}\right)\left(\epsilon_{p} \frac{R h}{2}+R^{2}\right)
$$

According to the perturbation method these equations are utilized with real frequencies. At the resonant frequency $\omega=\omega_{u 0}$ the stored electric and magnetic energies are equivalent $\left(W_{e}=W_{m}\right)$. Then the quality factor can be calculated as

$$
Q_{u 0}=\omega_{u 0} \frac{2 W_{e}}{P_{0}}=\omega_{u 0} \frac{2 W_{m}}{P_{0}}
$$




\subsubsection{Cylindrical Cavities completely filled with homogeneous dielectrics}

As mentioned in the case of the rectangular cavities the solution of the electromagnetic fields inside a microwave cylindrical cavity filled with a dielectric, with lossless cavity walls, is the same than for the empty cavity case or the case of a cavity with a lossless dielectric. The resonances persist only for a stable lossless medium, i.e. $\tan \delta=0$, or if an energy supply compensates the fields in lossy media. The resonant frequencies are expressed as for the case of the empty cavity (Equations 4.50 and 4.51 ) for the TE and TM modes replacing $\varepsilon_{0}$ with $\varepsilon_{0} \varepsilon_{r}(1-j \tan \delta)$ and taking the real part of the complex frequency:

$$
\begin{aligned}
& f_{u, m n p}=\frac{1}{2 \pi \sqrt{\mu_{0} \varepsilon_{0} \varepsilon_{r}}} \sqrt{\left(\frac{P_{m n}^{\prime}}{R}\right)^{2}+\left(\frac{p \pi}{h}\right)^{2}} \\
& f_{u, m n p}=\frac{1}{2 \pi \sqrt{\mu_{0} \varepsilon_{0} \varepsilon_{r}}} \sqrt{\left(\frac{P_{m n}}{R}\right)^{2}+\left(\frac{p \pi}{h}\right)^{2}}
\end{aligned}
$$

that is $f_{u}=f_{u 0} / \sqrt{\varepsilon_{r}}$.

The $Q$-factor is calculated as in the case of the rectangular cavity using Equations 4.31 - 4.34. Applying the electromagnetic field equations in the cylindrical cavity (Equations 4.44 and 4.47), the average stored electric and magnetic energies for the TE modes (with $m=0,1,2, \ldots, n=1,2,3, \ldots$ and $p=1,2,3, \ldots)$ are:

$$
\begin{aligned}
& W_{e}=\frac{\varepsilon_{0} \varepsilon_{r}}{16} \pi \epsilon_{m} \frac{A_{m n}^{2} \omega^{2} \mu_{0}^{2} R^{4} h}{P_{m n}^{\prime 2}}\left(1-\frac{m^{2}}{P_{m n}^{\prime 2}}\right) J_{m}^{2}\left(P_{m n}^{\prime}\right) \\
& W_{m}=\frac{\mu_{0}}{16} \pi \epsilon_{m} \frac{A_{m n}^{2} R^{2} h}{P_{m n}^{\prime 2}}\left(1-\frac{m^{2}}{P_{m n}^{\prime 2}}\right) J_{m}^{2}\left(P_{m n}^{\prime}\right) \cdot\left[\beta_{z}^{2} R^{2}+P_{m n}^{\prime 2}\right]
\end{aligned}
$$

and the dissipated power in the dielectric is read as

$$
P_{d}=\frac{\varepsilon_{0} \varepsilon_{r}}{8} \epsilon_{m} \pi \frac{A_{m n}^{2} \omega^{3} \mu_{0}^{2} R^{4} h}{P_{m n}^{\prime 2}}\left(1-\frac{m^{2}}{P_{m n}^{\prime 2}}\right) J_{m}^{2}\left(P_{m n}^{\prime}\right) \tan \delta
$$

In case of TM modes (with $m=0,1,2, \ldots, n=1,2,3, \ldots$ and $p=$ $0,1,2, \ldots)$ the stored electric and magnetic energies are given as

$$
W_{e}=\frac{\varepsilon_{0} \varepsilon_{r}}{16} \pi h \epsilon_{m} \epsilon_{p} \frac{B_{m n}^{2} R^{2} J_{m}^{\prime 2}\left(P_{m n}\right)}{P_{m n}^{2}}\left[P_{m n}^{2}+\beta_{z}^{2} R^{2}\right]
$$




$$
W_{m}=\frac{\mu_{0}}{16} \pi \epsilon_{m} \epsilon_{p} \frac{B_{m n}^{2} \omega^{2} \varepsilon_{0}^{2} \varepsilon_{r}^{2} R^{4} h}{P_{m n}^{2}} J_{m}^{\prime 2}\left(P_{m n}\right)
$$

and the dissipated power is

$$
P_{d}=\frac{\varepsilon_{0} \varepsilon_{r}}{8} \omega \pi h \epsilon_{m} \epsilon_{p} \frac{B_{m n}^{2} R^{2} J_{m}^{\prime 2}\left(P_{m n}\right)}{P_{m n}^{2}}\left[P_{m n}^{2}+\beta_{z}^{2} R^{2}\right] \tan \delta
$$

At the resonant frequency $\omega=\omega_{u}$, as in the case of the empty cavity, the stored electric and magnetic energies are equivalent $\left(W_{e}=W_{m}\right)$. The quality factor of the cavity filled with the dielectric can then be calculated as

$$
Q_{u}=\omega_{u} \frac{2 W_{e}}{P_{d}}=\omega_{u} \frac{2 W_{m}}{P_{d}}
$$

When the cavity filled with the dielectric has lossy walls, the cavity $Q-$ factor is calculated with Equation 4.35, being the same expression than for the $Q$-factor of the empty cavity $\left(Q_{u 0}\right)$ but calculated for the frequency of the perturbed cavity $w_{u} . P_{0}$ is calculated with Equation 4.22 also including $w_{u}$ in the calculation of $R_{s}$. Then, for the TE and TM modes the dissipated power on the walls of the cavity reads respectively

$$
\begin{gathered}
P_{0}=\frac{R_{s}}{2} \epsilon_{m} \pi A_{m n}^{2} \cdot J_{m}^{2}\left(P_{m n}^{\prime}\right)\left[\frac{R h}{2}\left(1+\frac{m^{2} \beta_{z}^{2} R^{2}}{P_{m n}^{\prime 4}}\right)+\frac{\beta_{z}^{2} R^{4}}{P_{m n}^{\prime 2}}\left(1-\frac{m^{2}}{P_{m n}^{\prime 2}}\right)\right] \\
P_{0}=\frac{R_{s}}{2} \epsilon_{m} \pi B_{m n}^{2} \omega^{2} \varepsilon_{0}^{2} \varepsilon_{r}^{2} \frac{1}{k_{c}^{2}} J_{m}^{\prime 2}\left(P_{m n}\right)\left(\epsilon_{p} \frac{R h}{2}+R^{2}\right)
\end{gathered}
$$

The total $Q$-factor of the cavity is defined as

$$
Q_{u}=\left.\omega \frac{W_{e}+W_{m}}{P_{t}}\right|_{\omega=\omega_{u}}
$$

where $P_{t}=P_{0}+P_{d}$ is the real dissipated power in the cavity, i.e. the sum of the dissipated power on the walls of the cavity and the dissipated power on the dielectric. The relation between $Q$-factors is then

$$
\frac{1}{Q_{u}}=\frac{1}{Q_{c}}+\frac{1}{Q_{d}}
$$




\subsection{Cavity coupling}

The coupling of a cavity is a mechanism which provides energy to the resonator. It generates an electromagnetic field, modelled as an electric or magnetic dipole moment, which couples the field of the resonant mode.

The coupling system always modifies the behaviour of the cavity and the generated fields with respect to the ideal case, i.e. the original unloaded frequency response is shifted to $f_{L}$ and the quality factor is lowered to $Q_{L}$ [58]. There are two kind of coupling networks: magnetic and electric.

Figure 4.2 represents a scheme of two kinds of magnetic networks: current loops and inductive iris. These networks couple magnetic fields and the resonance of the coupling network has an inductive behaviour. The coupling is positioned in zones where the magnetic field is maximal, with the dipole moment oriented in the direction of the magnetic field of the excited mode. The coupling strength depends on the magnetic flux through the loop i.e. the inductivity of the loop.

The question whether a loop coupling or an iris coupling is used lies in the applied power levels. Loop coupling is usually used at low powers while the iris allows the coupling of power levels sufficient for heating materials inside the cavity.

Electric coupling networks are modelled as electric dipole moments. The resonance of the coupling network has a capacitive behaviour. These

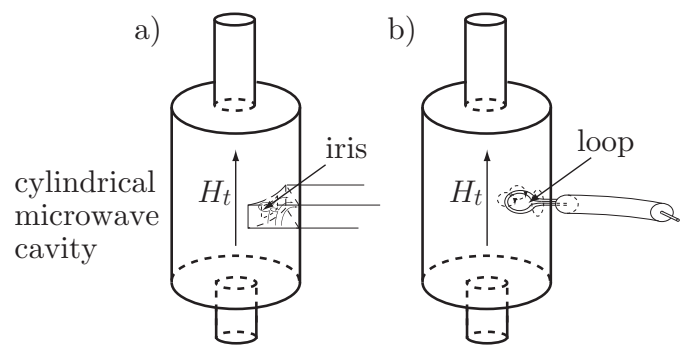

Figure 4.2. Different schemas of a magnetic coupling on a cylindrical microwave cavity: a) through an iris, b) through a loop. 


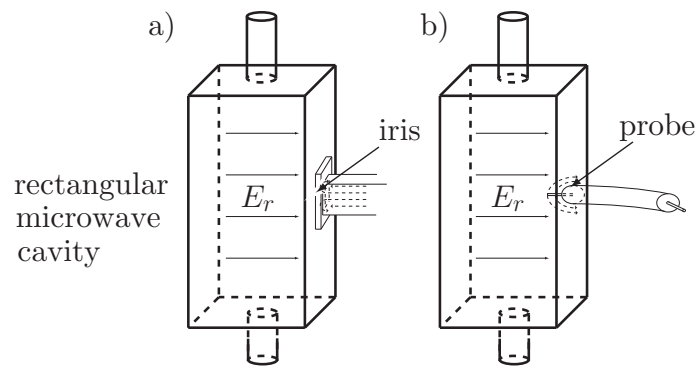

Figure 4.3. Different schemas of an electric coupling on a rectangular microwave cavity: a) through an iris, b) through an electric probe.

networks are placed in zones where the electric field is maximal with the dipole moment orientated in the direction of the electric field of the mode which is going to be excited. Some examples, shown in Figure 4.3 are electric probes and capacitive iris.

The degree of coupling must be known in order to calculate the unloaded parameters $Q_{u 0}$ and $f_{u 0}$ in empty cavities as well as $Q_{u}$ and $f_{u}$ in cavities with dielectrics. Several methods, which are based on equivalent circuits derived from Foster's forms, describe the extraction of those unloaded parameters from one-port measurements of resonators [58-60].

\subsubsection{The Coupling Factor}

The coupling factor is a parameter, with which the reflection coefficient at resonance is related by [49]

$$
\Gamma=\frac{1-\left(1 / \beta^{\prime}\right)}{1+\left(1 / \beta^{\prime}\right)}
$$

At critical coupling $\left(\beta^{\prime}=1\right)$ the reflection coefficient is zero $(\Gamma=0)$ whereas if the cavity is highly overcoupled $\left(\beta^{\prime} \gg 1\right)$ the coefficient tends to +1 and if highly undercoupled $\left(\beta^{\prime} \ll 1\right)$ tends to -1 .

The reflection parameter is represented with a circumference in a Smith chart, shown in Figure 4.4, where the diameter is dictated by the relation 


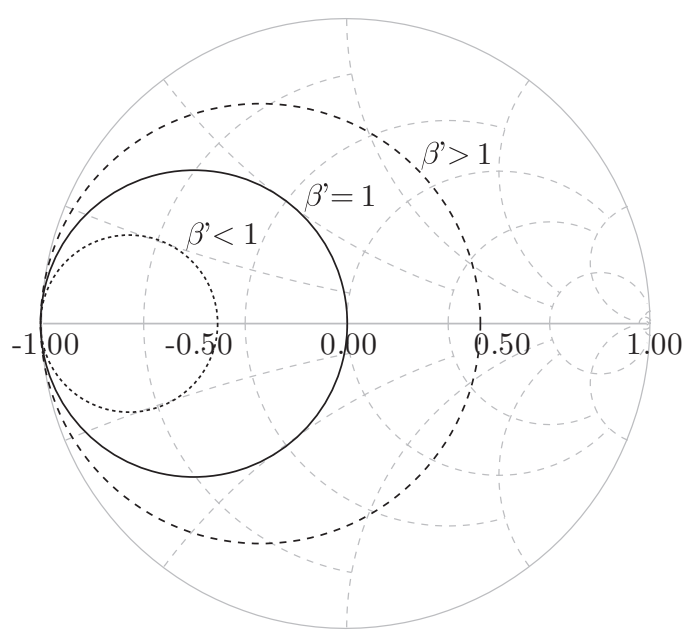

Figure 4.4. Smith chart representation of the reflection coefficient for an undercoupled $\left(\beta^{\prime} \ll 1\right)$, critically coupled $\left(\beta^{\prime}=1\right)$ and overcoupled $\left(\beta^{\prime} \gg 1\right)$ cavity.

with the coupling factor, and the position depends on the transmission line length.

When matching the cavity at its resonant frequency containing the sample, which is going to be measured or heated, it is recommended to work in a critical coupling situation, since it will provide the energy transfer from the generator to the workload with a minimum loss.

The ratio of the power dissipated in the external circuit to the power dissipated in the unloaded resonator gives the coupling factor, which is then related with the corresponding quality factors by [58]

$$
\beta^{\prime}=\frac{P_{e x}}{P_{0}}=\frac{Q_{u}}{Q_{e}}
$$

With the external quality factor, the detuning of a resonator due to the coupling elements can be calculated with the following expression [58]:

$$
f_{L}=\left(1+\frac{A}{Q_{e}^{\alpha}}\right) f_{u}
$$

where parameters $A$ and $\alpha$ depend on the electromagnetic fields inside the cavity, the coupling network and on the changes in the feeding network 
and modify $Q_{e}$. Since $f_{L}$ depends on $Q_{e}, A$ and $\alpha$ can be determined from several measurements of the input reflection coefficient by changing stepwise the feeding structure, e.g. different radii apertures or different loop insertion distance in the cylindrical cavity.

Coupling details referred to the PINE project are presented in Chapters 5 and 6.

\subsection{Modelling of Microwave Cavities}

Several software packages have been utilized to model the microwave cavities employed in the PINE project. These are presented and evaluated in this manuscript. On one side a numerical computation software, i.e. MATLAB ${ }^{\circledR}$, has been used to evaluate the fields of the different modes inside a cavity of determined dimensions. On the other side two electromagnetic simulation software packages offered the possibility to visualize the cavity structure as well as to calculate parameters such as resonant frequencies, field patterns, reflection coefficients, etc., for the predetermined modes.

\subsubsection{Matlab Modelling}

Different algorithms have been developed to model the fields inside a microwave cavity. Algorithm 4 computes the fields inside a rectangular cavity by using Equations 4.13 and 4.14 . 


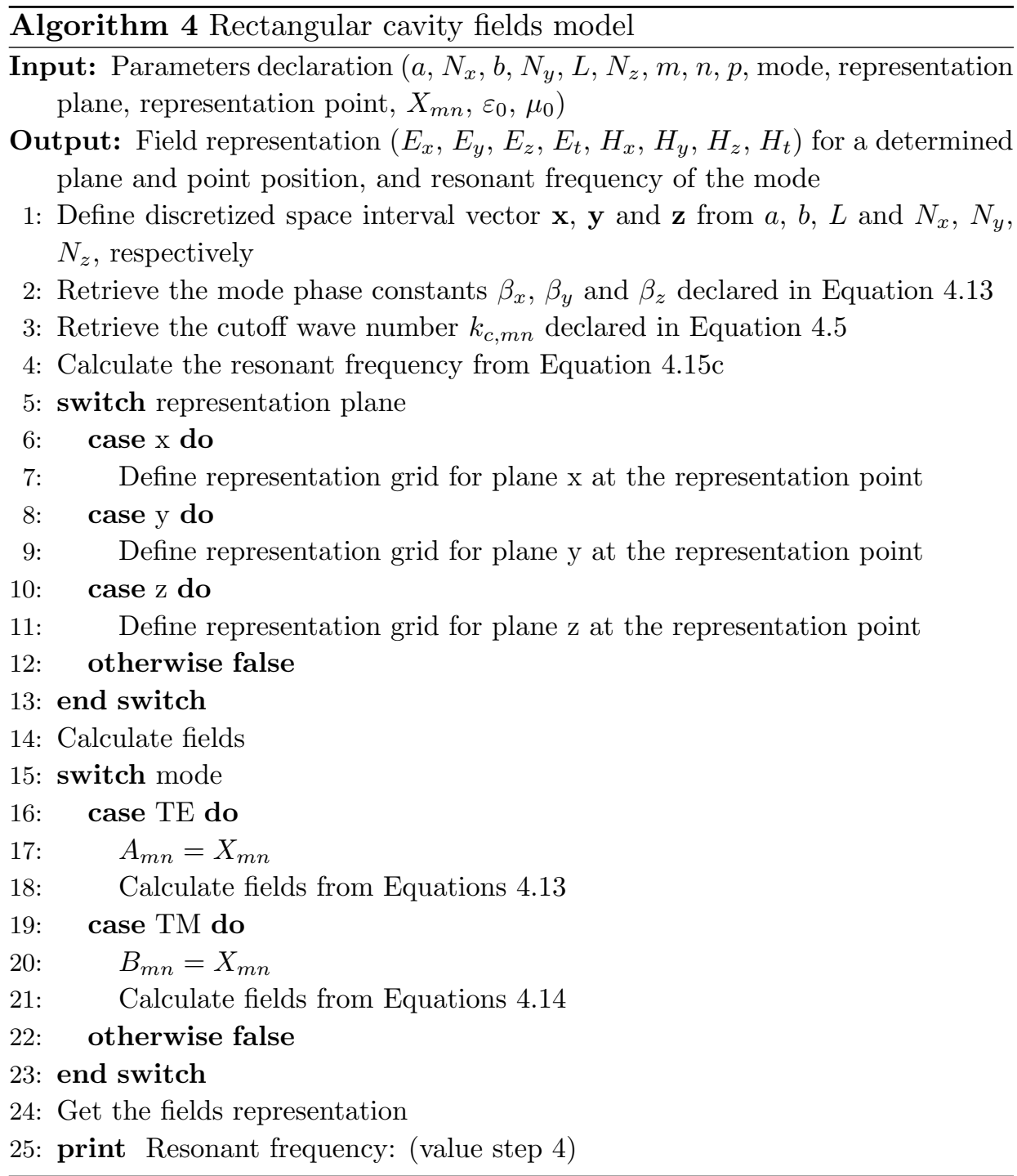


One of the input parameters is $X_{m n}$, which refers to the $A_{m n}$ (for $\mathrm{TE}_{m n p}$ modes) and $B_{m n}$ (for $\mathrm{TM}_{m n p}$ modes) constants presented in the field equations. These constants are related with the incident power $\left(P_{0}\right)$ and the metal conductivity, and they are solved before the fields are determined with Algorithm 4. Algorithm 5 resumes the code for its calculation.

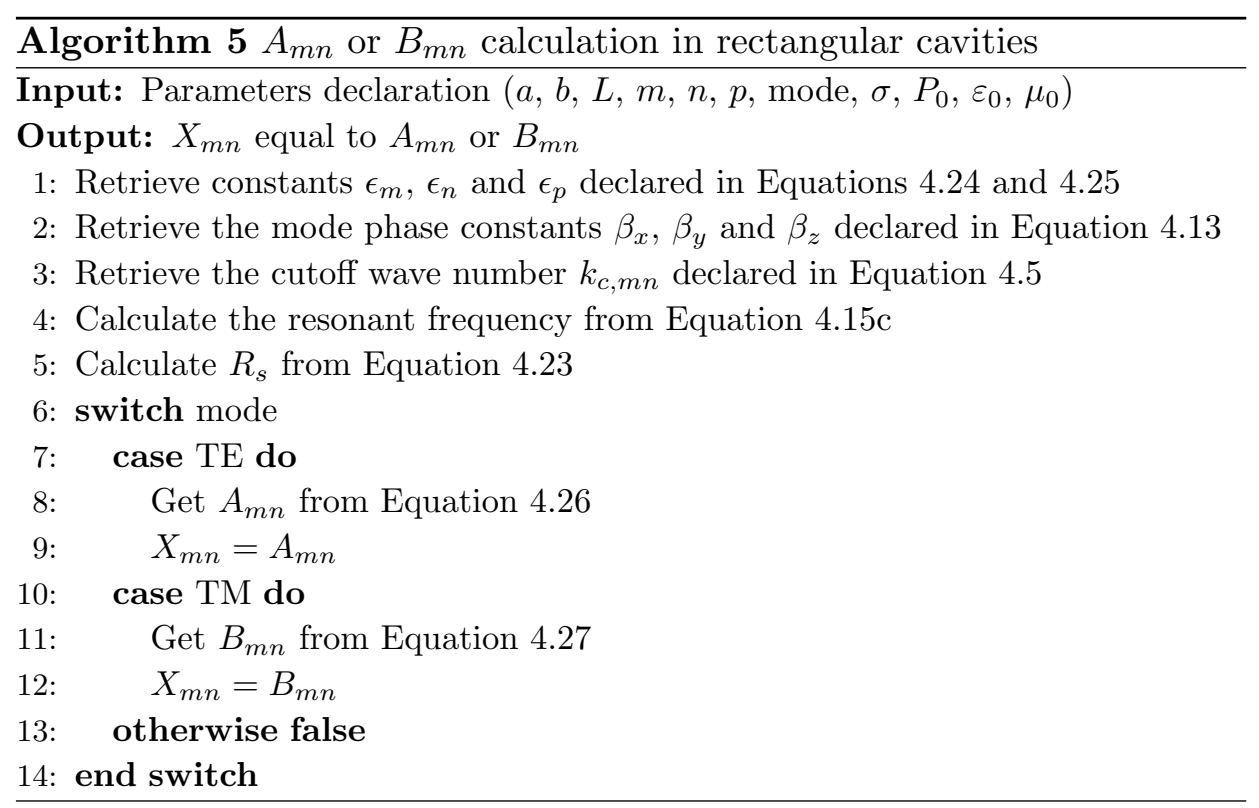

Figure 4.5 shows the order of resonant frequencies of the TE and TM modes as function of the cavity length for a cavity which dimensions $a \times b$ correspond to the standard X-band WR90 waveguide dimensions $(22.86 \times$ $10.16 \mathrm{~mm}$ ). The frequencies has been calculated by processing iteratively the Algorithms 4 and 5 for an input power of $10 \mathrm{~W}$ and a metal conductivity equal to $2.069 \mathrm{e} 7 \mathrm{~S} \cdot \mathrm{m}^{-1}$. 


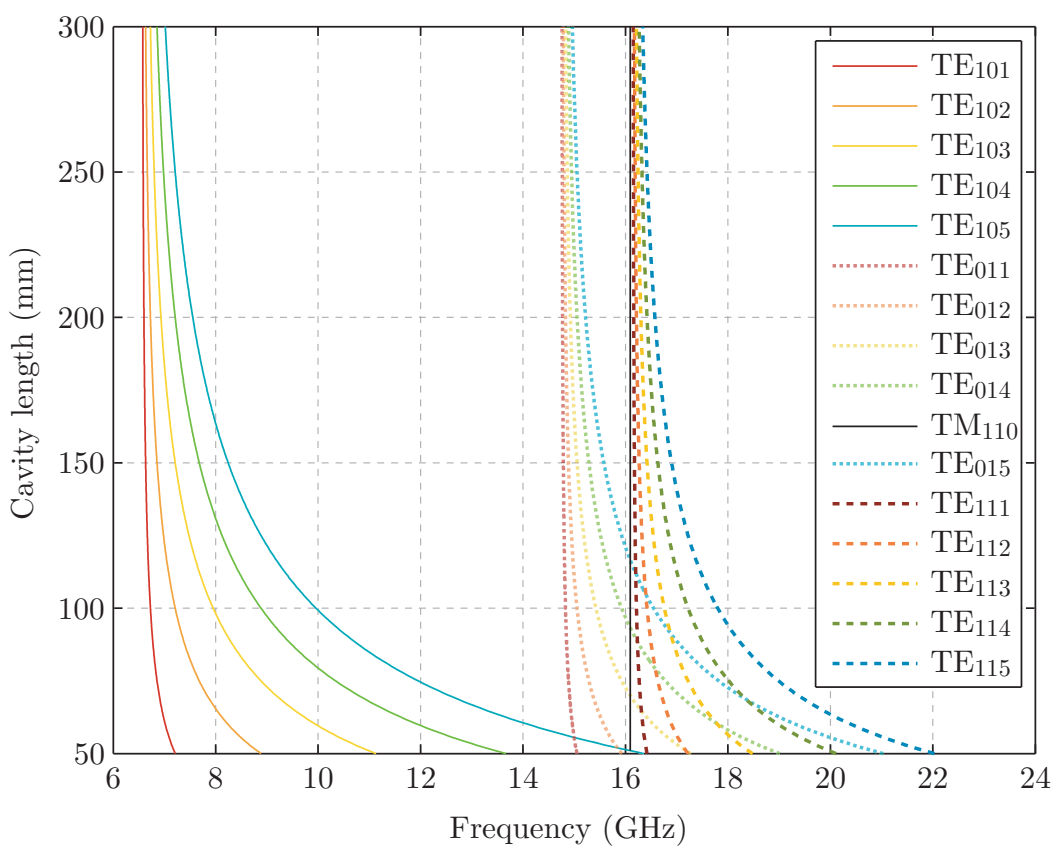

Figure 4.5. Rectangular cavity modes chart as a function of cavity length.

In case of the PINE project a cavity length of $200 \mathrm{~mm}$ has been selected. In this way, the cavity is large enough to heat the samples while passing through its central axis.

Figure 4.6 represents several $\mathrm{TE}_{m n p}$ and $\mathrm{TM}_{m n p}$ mode examples of the total electric field pattern generated inside an empty rectangular Xband cavity, the dimensions of which correspond to a WR-90 waveguide $(22.86 \times 10.16 \mathrm{~mm})$ of $200 \mathrm{~mm}$ length. The representations are cut views of the $x z$ plane taken at the middle of the cavity height $y=b / 2$. 
a) $\quad \mathrm{TE}_{101}$

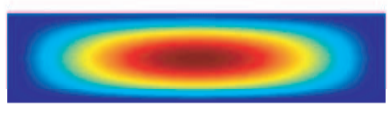

c) $\quad \mathrm{TE}_{011}$

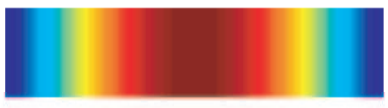

e) $\quad \mathrm{TE}_{111}$

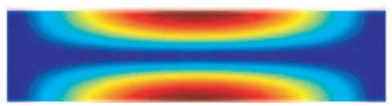

g) $\quad \mathrm{TE}_{211}$

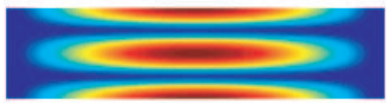

i)

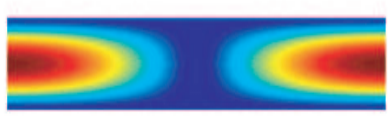

k)

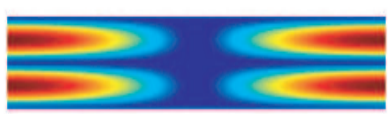

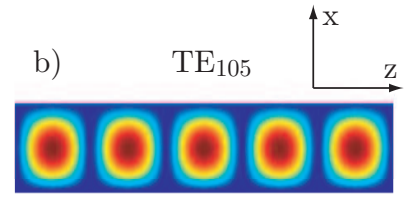

d) $\quad \mathrm{TE}_{015}$

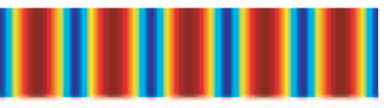

f) $\quad \mathrm{TE}_{115}$

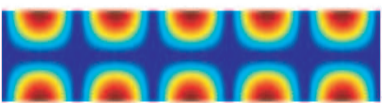

h) $\quad \mathrm{TE}_{215}$

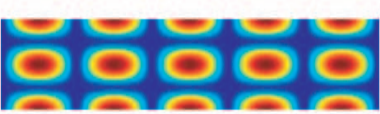

j) $\quad \mathrm{TM}_{115}$

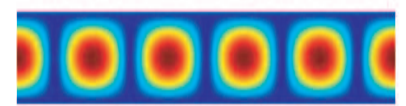

1) $\quad \mathrm{TM}_{215}$

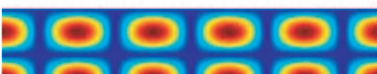

Figure 4.6. Total E-field pattern of several $\mathrm{TE}_{m n p}$ and $\mathrm{TM}_{m n p}$ modes inside a rectangular cavity of $22.86 \times 10.16 \times$ $200 \mathrm{~mm}$ (along $x, y, z$ ) dimensions. View of the centered $x z$ plane

In the PINE project the mode $\mathrm{TE}_{10(15)}$ at $13.0147 \mathrm{GHz}$ has been selected. $\mathrm{TE}_{10 p}$ modes ensure the maximums of the field only along the central axis of the microwave cavity (in $z$ ). Then the drops passing through have maximal interaction with the field. The mode with $p=15$ gives the 


\begin{tabular}{ll}
\hline Width & $\mathrm{a}=22.86 \mathrm{~mm}$ \\
Height & $\mathrm{b}=10.16 \mathrm{~mm}$ \\
Length & $\mathrm{L}=200 \mathrm{~mm}$ \\
Mode & $\mathrm{TE}_{10(15)}$ \\
Metal Conductivity & $\sigma=2.069 \mathrm{e} \mathrm{S} \cdot \mathrm{m}^{-1}$ \\
Frequency & $13.0147 \mathrm{GHz}$ \\
\hline
\end{tabular}

Table 4.1. PINE cavity parameters.

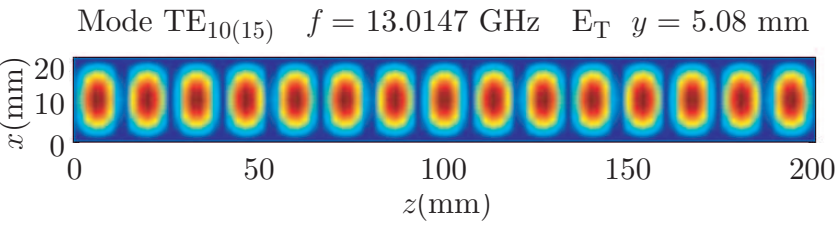

Figure 4.7. Total E-field pattern of the $\mathrm{TE}_{10(15)}$ mode inside the PINE rectangular cavity. View of the centered $x z$-plane

maximal frequency in the X-band range and it is not disturbed with higher modes frequencies. Table 4.1 summarizes the parameters of the PINE microwave cavity design.

Figure 4.7 shows the total E-field pattern of the selected mode for the experiments with the PINE microwave cavity.

Algorithms 6 and 7 model the fields inside a cylindrical empty microwave cavity from the dimensions and the mode specifications by using Equations 4.44 and 4.47 in a cylindrical coordinates system.

The input parameter $X_{m n}$, is computed from the incident power $\left(P_{0}\right)$ and the metal conductivity, following the code in Algorithm 7. 


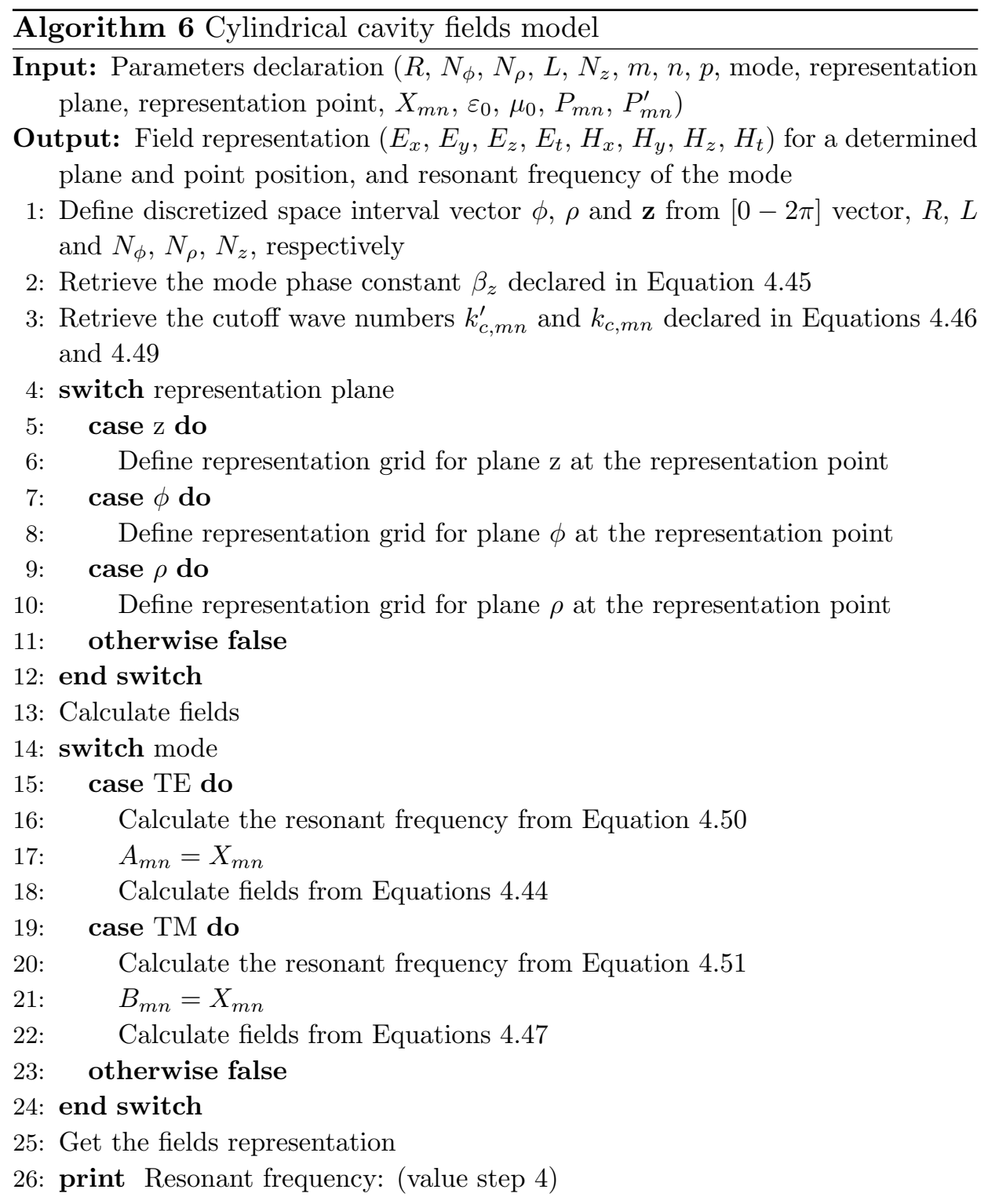




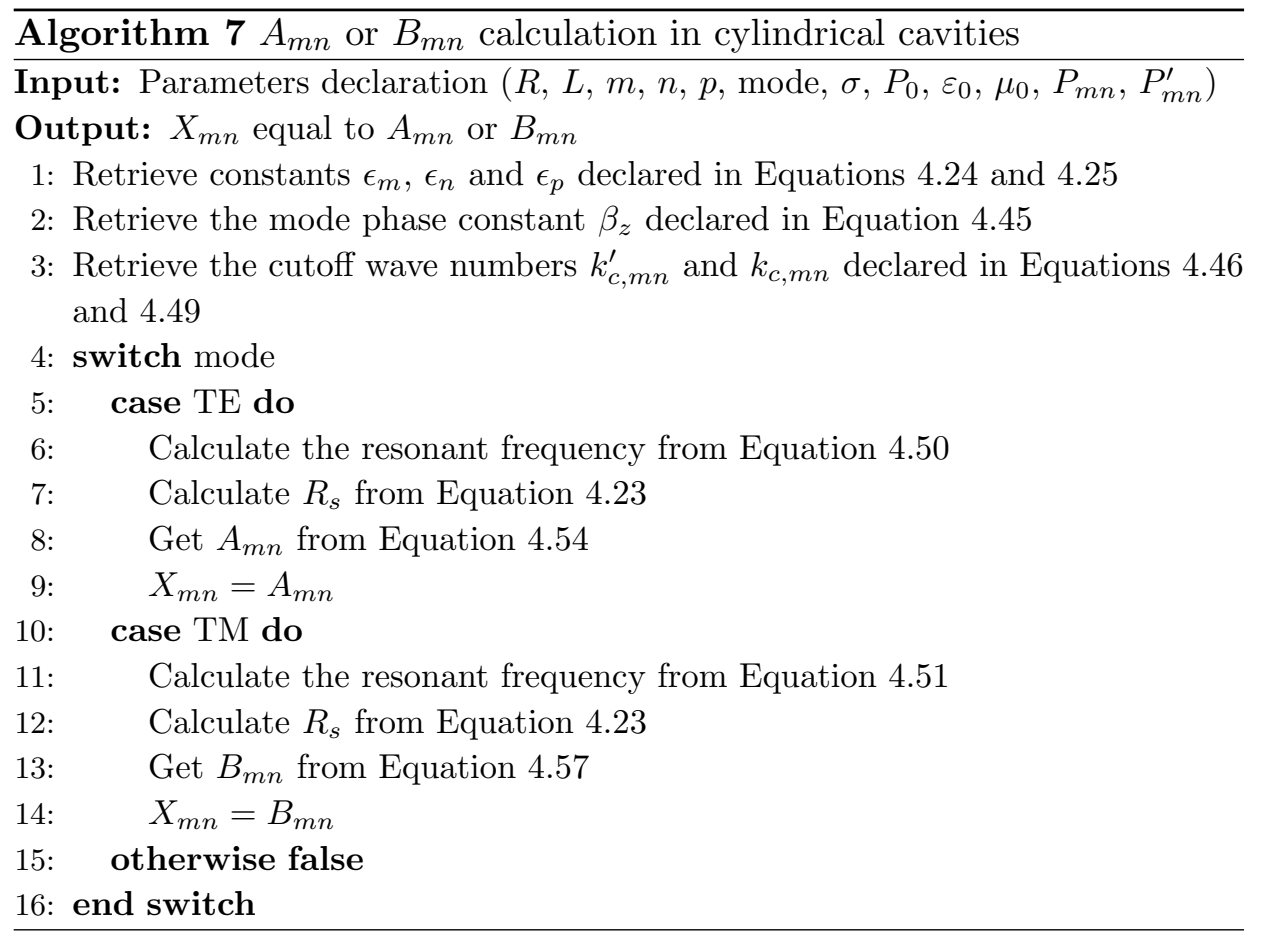


Figures 4.8 and 4.9 show the chart of TE and TM modes respectively. The diameter of the cylindrical cavity $(18 \mathrm{~mm})$ has been selected to find the frequencies of the modes in the X-band range. The resonant frequencies (closer in the spectra for larger cavities) have been calculated by processing iteratively the Algorithms 6 and 7 for $P_{0}=10 \mathrm{~W}$ and $\sigma=2.069 \mathrm{e} \mathrm{S} \cdot \mathrm{m}^{-1}$.

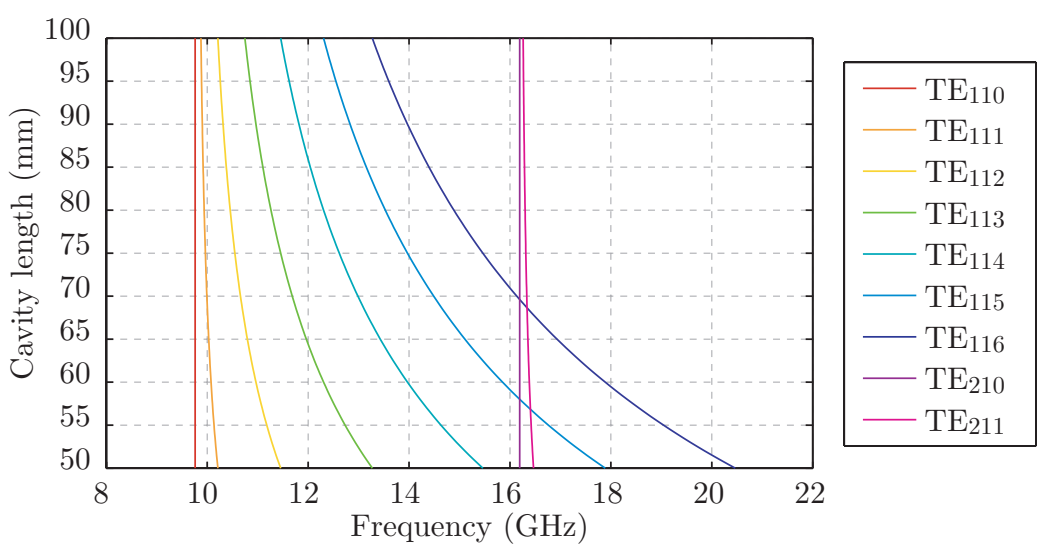

Figure 4.8. Cylindrical cavity TE modes chart (9 mm radius).

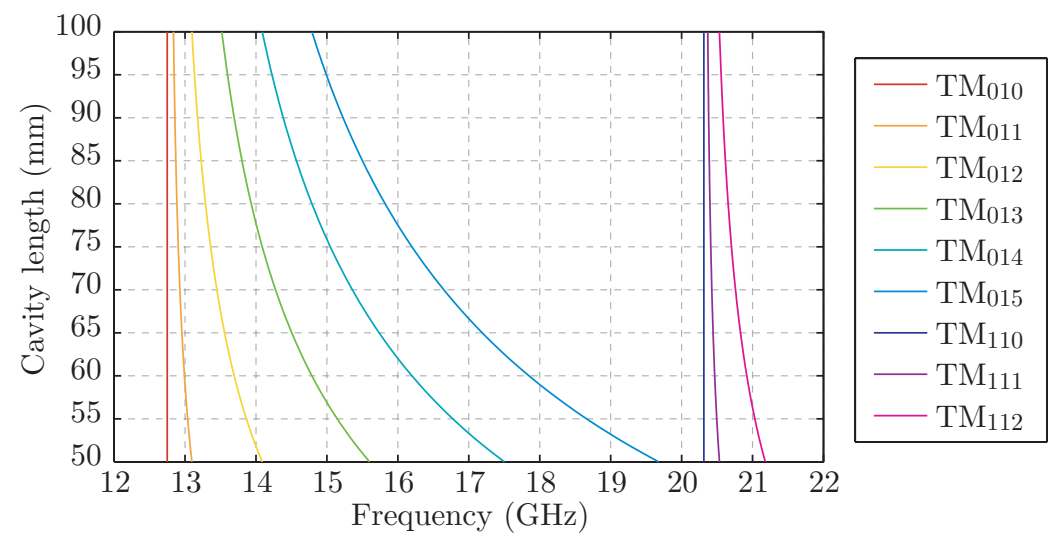

Figure 4.9. Cylindrical cavity TM modes chart (9 $\mathrm{mm}$ radius). 
a) $\mathrm{TE}_{111}$

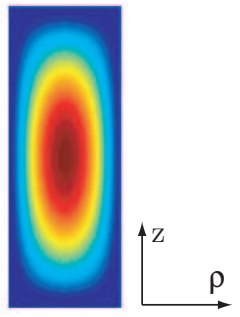

b) $\mathrm{TE}_{112}$

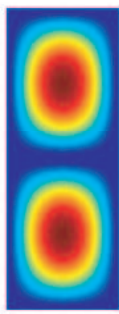

e) $\mathrm{TE}_{212}$

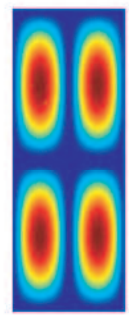

i) $\mathrm{TM}_{012}$

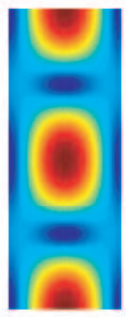

f) $\mathrm{TE}_{213}$

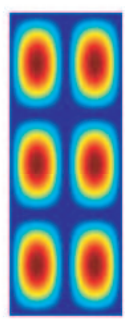

j) $\mathrm{TM}_{013}$

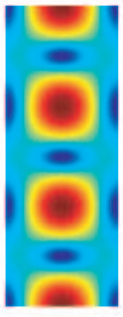

c) $\mathrm{TE}_{113}$

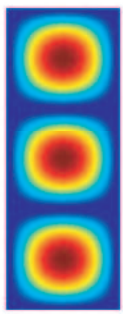

g) $\mathrm{TM}_{010}$

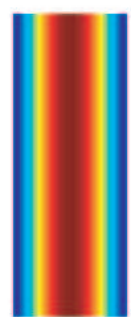

k) $\mathrm{TM}_{110}$

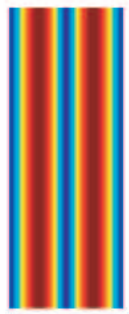

d) $\mathrm{TE}_{211}$

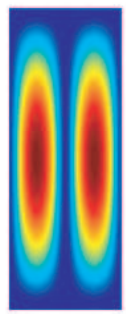

h) $\mathrm{TM}_{011}$

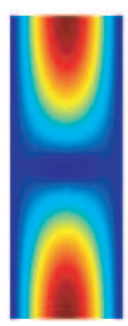

1) $\mathrm{TM}_{111}$

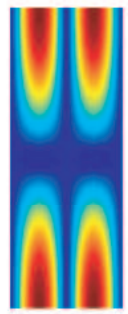

Figure 4.10. Total E-field pattern of several $\mathrm{TE}_{m n p}$ and $\mathrm{TM}_{m n p}$ modes inside a cylindrical $9 \mathrm{~mm}$ radius cavity of $50 \mathrm{~mm}$ length. View of the middle $(\phi=0)$-plane

In case of the PINE project, a cavity length of $50 \mathrm{~mm}$ has been selected looking for the longest residence time while avoiding interference with high order modes in the same frequency band. Figure 4.10 represents the total 
electric field patterns generated inside an empty cylindrical X-band cavity for several $\mathrm{TE}_{m n p}$ and $\mathrm{TM}_{m n p}$ mode examples. The cavity dimensions are $9 \mathrm{~mm}$ radius and $50 \mathrm{~mm}$ length. The representations are a cut view of the $\rho z$ plane taken in the $\phi=0$ point.

The $\mathrm{TM}_{010}$ mode at $12.8267 \mathrm{GHz}$ is the one utilised in the dielectric properties measurements of the PINE project due to its constant field pattern along the central axis (in z) where the samples pass through.

\subsubsection{Electromagnetic Simulation Software}

QuickWave-3D is a three-dimensional electromagnetic simulator based on the Finite Difference Time Domain (FDTD) method and supplemented with a range of unique models for curved boundaries, media interfaces, modal excitation, and parameter extraction [61].

It can be applied to a variety of microwave problems including: accurate S-parameter determination, calculation of heating patterns for microwave power applications, with accurate and fast display of instantaneous, time-maximum, and time-averaged patterns of fields and dissipated power, determination of eigenfrequencies, Q-factors, and pure modal field patterns for shielded and open inhomogeneous resonators, also in cases involving closely-spaced modes.

CST is also a three-dimensional electromagnetic simulation software which offers accurate, efficient computational solutions for electromagnetic design and analysis and enables to choose the most appropriate method for the design and optimization of devices operating in a wide range of frequencies [62].

CST MICROWAVE STUDIO ${ }^{\circledR}$ (CST MWS) is a specialist tool for the three-dimensional electromagnetic simulation of high frequency components, which gives an insight into its behaviour. From the six different solver CST MWS modules, the time domain solver applies the Perfect Boundary Approximation $(\mathrm{PBA})^{\circledR}$, which are modules based on FEM, MoM, MLFMM and SBR methods. This transient solver also delivers frequency domain results like S-parameters with a fine frequency resolution. 


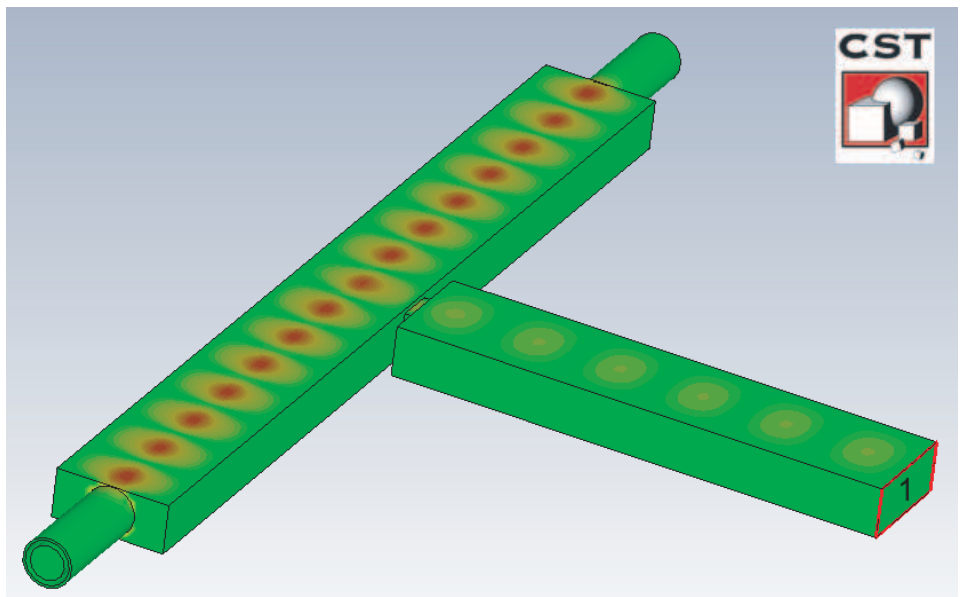

Figure 4.11. Simulation of the E-field distribution in the rectangular PINE cavity performed with CST software

Compared to the Matlab modelling, the electromagnetic simulation software allows the evaluation of the coupling and other mechanisms, e.g. cavity openings for the transfer of samples. The behaviour of the fields when interacting with samples inside the cavity can also be simulated. Figure 4.11 show the simulation of the PINE cavity described in Table 4.1 performed with CST software. In this simulation the cavity is fed from a $\mathrm{X}$-band waveguide through a rectangular iris. Furthermore the apertures for the insertion of the drops and the quartz tube, which prevents the cavity from contamination, are also simulated.

It can be seen that the E-field pattern is close to the one calculated with the Matlab modelling (represented in Figure 4.7).

The use of both software tools referred to the PINE project are further presented in Chapters 5 and 6. 


\subsection{Perturbation Analysis of Cavities}

As mentioned in Chapter 1, in the motivation part (Section 1.2), the PINE project aims for the production of SP-fuel by MIG. The related literature has been presented in Chapter 2 (Section 2.5). Within this section the selection of the X-band as a best candidate frequency range for the design of a heating microwave cavity in the gelation process is proved. First the perturbation method shows how the frequency is deviated when a sample is introduced in a cavity. Afterwards the power dissipation model is analysed, i.e. when a drop, seen as a small spherical specimen, is falling down through the central axis of a $\mathrm{TE}_{10 p}$ rectangular cavity. The perturbation analysis can be applied if the variation of the field produced by the dielectric is minimal, which is the case of the samples in the PINE project.

\subsubsection{Experimental setup}

Figure 4.12 shows a schematic view of the installation, for which simulation the perturbation method is applied. A microwave rectangular cavity is used to evaluate the power dissipated when a drop passes through its central axis. The cavity dimensions, operating mode and frequency correspond to the ones selected for the PINE project as described in the previous section (Table 4.1).

A $1.1 \mathrm{~mm}$ radius drop is used as a specimen to be heated in the cavity. For the calculations the drop is approximated by a cube of a side length $c$ equal to the cube root of the volume of the drop as

$$
c=\left(\frac{4}{3} \pi R^{3}\right)^{1 / 3}
$$

where $R$ is the drop radius, which is considered as a sphere, because of the high surface tension.

\subsubsection{Perturbation Method}

Usually the perturbation theory provides the key to calculate the dielectric properties of a material inserted in a microwave cavity. However, knowing the complex permittivity and permeability, $\varepsilon_{r}=\varepsilon^{\prime}-j \varepsilon^{\prime \prime}$ and $\mu_{r}=\mu^{\prime}-j \mu^{\prime \prime}$, 


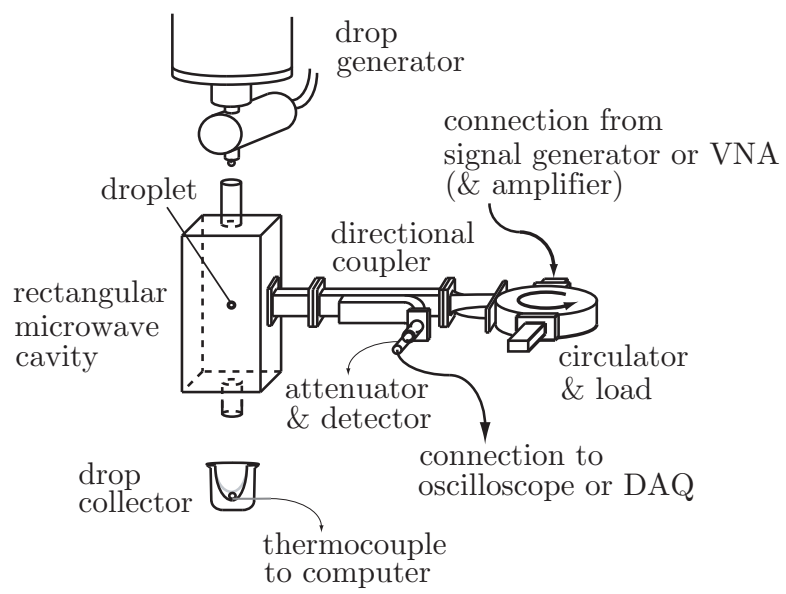

Figure 4.12. MIG Setup (B and C).

respectively, this theory can be useful to calculate the dissipated power in a material from the complex resonant frequency shift, and from the relations between sample dimension, position and electromagnetic properties. The shift of the complex resonant frequency can be written as [63-65]

$$
\frac{\Omega_{u}-\Omega_{u 0}}{\Omega_{u}}=-\frac{\int_{v_{s}}\left[\varepsilon_{0}\left(\varepsilon_{r}-1\right) E \cdot E_{0}-\mu_{0}\left(\mu_{r}-1\right) H \cdot H_{0}\right] d v}{\int_{V}\left[\varepsilon_{0} \varepsilon_{r} E_{0} \cdot E-\mu_{0} \mu_{r} H_{0} \cdot H\right] d v}
$$

where $E_{0}$ and $H_{0}$ are the electric and magnetic fields in the empty cavity, $E$ and $H$ are the electric and magnetic fields in the cavity perturbed with the sample, and $v_{s}$ and $V$ are the volumes of the sample and the cavity respectively.

The complex resonance frequencies can be expressed in terms of measurable quantities as

$$
\Omega_{n}=\omega\left(1+j \frac{1}{2 Q_{n}}\right)
$$

where the index changes referring in each case to the perturbed or unperturbed situation. 
For specimens with the same permeability than the original medium in the cavity or specimens placed in a region where the magnetic field is negligible (i.e. in a maximum of electric field), the variation of the complex resonance frequency (Equation 4.76) can be written [63]

$$
\frac{\Omega_{u}-\Omega_{u 0}}{\Omega_{u}}=-\frac{\left(\varepsilon_{r}-1\right) \int_{v_{s}} E \cdot E_{0} d v}{2 \int_{V}\left|E_{0}\right|^{2} d v}
$$

where perturbation conditions are assumed: the volume of the sample is very small with respect the wavelength $v_{s} \ll V$ and its insertion produces only a small change of the fields [66], i.e. the electromagnetic field with and without the sample are approximately equal (the relative shift of the frequency is small and the changes in the inverse of $Q$-factor is less than the relative shift of the frequency [67]).

Although it would be possible to work directly with unperturbed and perturbed complex frequencies, $\Omega_{u 0}$ and $\Omega_{u}$, it is usual to split Equation 4.78 into its real and imaginary parts by making use of 4.77 , so the left side is expressed in terms of measurable quantities as

$$
\frac{\Omega_{u}-\Omega_{u 0}}{\Omega_{u}}=\frac{f_{u}-f_{u 0}}{f_{u}}+j \frac{f_{u 0}}{f_{u}} \frac{1}{2}\left(\frac{1}{Q_{u}}-\frac{1}{Q_{u 0}}\right)
$$

and then

$$
\begin{aligned}
& \frac{\Delta f}{f}=-\eta\left(\varepsilon^{\prime}-1\right)=\frac{f_{u}-f_{u 0}}{f_{u}} \\
& \Delta\left(\frac{1}{2 Q}\right)=\eta \varepsilon^{\prime \prime}=\frac{f_{u 0}}{f_{u}} \frac{1}{2}\left(\frac{1}{Q_{u}}-\frac{1}{Q_{u 0}}\right)
\end{aligned}
$$

where the filling factor $\eta$ is given by

$$
\eta=\frac{\int_{v_{s}}\left|E_{0}\right|^{2} d v}{2 \int_{V}\left|E_{0}\right|^{2} d v}
$$

The electric field inside the specimen, $E$, is not known. Because the drops are considered to be spheres appear consequently depolarization effects and $E$ can not be considered equal to $E_{0}$ but related to it and to the permittivity and shape of the sample. For ellipsoidal samples immersed in 
a uniform field parallel to one of the axes of the ellipsoid, the internal field can be expressed, in a quasi static (QS) approximation, as [68]

$$
E=\frac{E_{0}}{1+N\left(\varepsilon_{r}-1\right)}
$$

where $N \in[0,1]$ is the sample depolarization factor in the direction of the electric field polarization. In the case of a sphere, $N=1 / 3$ [69]. Applying the depolarization factor to Equations 4.80 and 4.81 the variation of the frequency and $Q$-factor while the sample is passing through the cavity is calculated, respectively, as

$$
\begin{aligned}
& \frac{\Delta f}{f}=-\eta \frac{\left(\varepsilon^{\prime}-1\right)\left[1+N\left(\varepsilon^{\prime}-1\right)\right]+N \varepsilon^{\prime \prime 2}}{\left[1+N\left(\varepsilon^{\prime}-1\right)\right]^{2}+\left(N \varepsilon^{\prime \prime}\right)^{2}} \\
& \Delta\left(\frac{1}{2 Q}\right)=\frac{\eta \varepsilon^{\prime \prime}}{\left[1+N\left(\varepsilon^{\prime}-1\right)\right]^{2}+\left(N \varepsilon^{\prime \prime}\right)^{2}}
\end{aligned}
$$

The filling factor, which depends on the cavity volume and the samples volume, is solved applying the field equations in two parts: $\eta_{\text {num }}$, which refers to the numerator of Equation 4.82 i.e. the integration of the field in the sample volume, and $\eta_{\text {den }}$, which refers to the denominator of 4.82 i.e. the integration of the field in the cavity volume, then

$$
\eta=\frac{\eta_{\text {num }}}{2 \eta_{\text {den }}}
$$

For cavities working in $\mathrm{TE}_{10 p}$ modes, $\eta_{\text {den }}$ is calculated as

$$
\begin{aligned}
& \int_{V}\left|E_{0}\right|^{2}=\int_{V} B_{m n}^{2} \frac{\left(\omega \mu_{0}\right)^{2}}{k_{c, m n}^{4}}\left[\frac{\pi}{a} \sin \left(\frac{\pi}{a} x\right) \sin \left(\frac{p \pi}{L} z\right)\right]^{2} d V \\
& =B_{m n}^{2} \frac{\left(\omega \mu_{0}\right)^{2}}{k_{c, m n}^{4}} \int_{V}\left[\left(\frac{\pi}{a}\right)^{2} \sin ^{2}\left(\frac{\pi}{a} x\right) \sin ^{2}\left(\frac{p \pi}{L} z\right)\right] d x d y d z \\
& =B_{m n}^{2} \frac{\left(\omega \mu_{0}\right)^{2}}{k_{c, m n}^{4}}\left(\frac{\pi}{a}\right)^{2} \int_{0}^{a} \sin ^{2}\left(\frac{\pi}{a} x\right) d x \int_{0}^{b} d y \int_{0}^{L} \sin ^{2}\left(\frac{p \pi}{L} z\right) d z \\
& =B_{m n}^{2} \frac{\left(\omega \mu_{0}\right)^{2}}{k_{c, m n}^{4}}\left(\frac{\pi}{a}\right)^{2} \frac{1}{2}\left[x-\frac{a}{2 \pi} \sin \left(\frac{2 \pi}{a} x\right)\right]_{0}^{a}[y]_{0}^{b} \frac{1}{2}\left[z-\frac{L}{2 \pi p} \sin \left(\frac{2 \pi p}{L} z\right)\right]_{0}^{L} \\
& =B_{m n}^{2} \frac{\left(\omega \mu_{0}\right)^{2}}{k_{c, m n}^{4}}\left(\frac{\pi}{a}\right)^{2} \frac{1}{2} a b \frac{1}{2} L
\end{aligned}
$$


The sample is falling down through the central axis of the cavity $(a / 2$, $b / 2, z)$. Along this axis $\eta_{\text {num }}$ will be solved, by the same procedure as $\eta_{\text {den }}$ for three different cases: when the sample is inside the cavity $(c / 2<$ $z<L-c / 2$ ), when the sample is entering the cavity, i e. top boundary $(-c / 2<z<c / 2)$ and when the sample is leaving the cavity, i.e. bottom boundary $(L-c / 2<z<L+c / 2)$.

Then, the solution of the filling factor is given in three parts:

- Inside the cavity $(c / 2<z<L-c / 2)$

$$
\eta=\frac{1}{2} \frac{c}{a b L}\left[c+\frac{a}{\pi} \sin \left(\frac{\pi}{a} c\right)\right]\left[c-\frac{L}{\pi p} \cos \left(\frac{2 \pi p}{L} z\right) \sin \left(\frac{\pi p}{L} c\right)\right]
$$

- Top boundary $(-c / 2<z<c / 2)$

$$
\eta=\frac{1}{2} \frac{c}{a b L}\left[c+\frac{a}{\pi} \sin \left(\frac{\pi}{a} c\right)\right]\left[z+\frac{c}{2}-\frac{L}{2 \pi p} \sin \left(\frac{2 \pi p}{L}\left(z+\frac{c}{2}\right)\right)\right]
$$

- Bottom boundary $(L-c / 2<z<L+c / 2)$

$$
\eta=\frac{1}{2} \frac{c}{a b L}\left[c+\frac{a}{\pi} \sin \left(\frac{\pi}{a} c\right)\right]\left[L-z+\frac{c}{2}+\frac{L}{2 \pi p} \sin \left(\frac{2 \pi p}{L}\left(z-\frac{c}{2}\right)\right)\right]
$$

\subsubsection{Power dissipation}

If the cavity is coupled to a microwave generator by means of a one-port lossless coupling mechanism, from [59] (p. 57), it is possible to approximate the magnitude of its reflection coefficient $|\Gamma|$ around a resonance by means of

$$
|\Gamma|=\left|1-2\left(\frac{Q_{e}}{Q_{u}}+1+2 j Q_{e} \frac{f_{g e n}-f_{u}}{f_{u}}\right)^{-1}\right|
$$

where $Q_{e}$ is the external $Q$-factor, i.e. external from the resonator, which considers the losses due to the coupling network, and $f_{\text {gen }}$ is the working frequency of the microwave generator. 
When the sample is passing through a maximum of E-field, both the resonance frequency and $Q$-factor of the resonator reach their maximum deviation from values of the empty case, i.e. their minimum values, $f_{u(\min )}$ and $Q_{u(\min )}$, respectively. In this situation it seems convenient to match the cavity to the generator $(\Gamma=0)$ and to tune the microwave generator frequency $f_{\text {gen }}$ to the resonance frequency of the cavity. So, by design, $f_{\text {gen }}=f_{u(\min )}$ and $Q_{e}=Q_{u(\min )}$. Then $|\Gamma|>0$ in any other position of the sample.

From the generator power $P_{\text {gen }}$, the transmitted power to the cavity can be obtained as

$$
P_{\text {in }}=P_{\text {gen }}\left(1-|\Gamma|^{2}\right)
$$

This power is dissipated by the cavity walls $\left(P_{0}\right)$ and by the dielectric sample $\left(P_{d}\right)$

$$
P_{\text {in }}=P_{0}+P_{d}
$$

where according to [56] the power dissipated by the cavity walls is calculated from Equation 4.22 and the power dissipated by the dielectric sample is calculated from Equation 4.33. Applying the depolarization factor leads to:

$$
P_{d}=\frac{\sigma_{e}}{2} \int_{v_{s}}|E|^{2} d v=\frac{\sigma_{e} / 2}{\left|1+N\left(\varepsilon^{\prime}-1-j \varepsilon^{\prime \prime}\right)\right|^{2}} \int_{v_{s}}\left|E_{0}\right|^{2} d v
$$

with

$$
\sigma_{e}=\omega \varepsilon_{0} \varepsilon^{\prime \prime}
$$

For the calculation of $P_{0}$ (solved in Equation 4.26) the tangential magnetic field in the cavity walls, $H_{\tan }$, is considered to have the same distribution than in the unperturbed cavity.

For the solution of the dissipated power by the dielectric the approximation of the drop as a equi-volume cube is also utilized. As in the case of the filling factor calculation $(\eta), P_{d}$ is solved for three different cases: inside the cavity, on the top boundary and on the bottom boundary. The solution reads: 
- Inside the cavity $(c / 2<z<L-c / 2)$

$$
P_{d}=\alpha_{P_{d}}\left(c-\frac{L}{\pi p} \cos \left(\frac{2 \pi p}{L} z\right) \sin \left(\frac{\pi p}{L} c\right)\right) \beta_{P_{d}}
$$

- Top boundary $(-c / 2<z<c / 2)$

$$
P_{d}=\alpha_{P_{d}}\left(z+\frac{c}{2}-\frac{L}{2 \pi p} \sin \left(\frac{2 \pi p}{L}\left(z+\frac{c}{2}\right)\right)\right) \beta_{P_{d}}
$$

- Bottom boundary $(L-c / 2<z<L+c / 2)$

$$
P_{d}=\alpha_{P_{d}}\left(L-z+\frac{c}{2}+\frac{L}{2 \pi p} \sin \left(\frac{2 \pi p}{L}\left(z-\frac{c}{2}\right)\right)\right) \beta_{P_{d}}
$$

where, $\alpha_{P_{d}}$ and $\beta_{P_{d}}$ encompass the common parts of the equations as

$$
\begin{gathered}
\alpha_{P_{d}}=\frac{1}{\left|1+N\left(\varepsilon_{r}-1\right)\right|} \frac{\sigma_{e}}{2} A_{m n}^{2} \frac{\left(\omega \mu_{0}\right)^{2}}{k_{c, m n}^{4}} \frac{1}{8} \\
\beta_{P_{d}}=\left[\left(\frac{n \pi}{b}\right)^{2} \epsilon_{m}\left(c+A^{\prime}\right)\left(c-B^{\prime}\right)+\left(\frac{m \pi}{a}\right)^{2} \epsilon_{m}\left(c-A^{\prime}\right)\left(c+B^{\prime}\right)\right]
\end{gathered}
$$

with,

$$
\begin{aligned}
A^{\prime} & = \begin{cases}0 & m=0 \\
\frac{a}{\pi m} \cos (\pi m) \sin \left(\frac{c \pi m}{a}\right) & m \neq 0\end{cases} \\
B^{\prime} & = \begin{cases}0 & n=0 \\
\frac{b}{\pi n} \cos (\pi n) \sin \left(\frac{c \pi n}{b}\right) & n \neq 0\end{cases} \\
\epsilon_{m} & = \begin{cases}2, & m=0 \\
1, & m \neq 0\end{cases} \\
\epsilon_{n} & = \begin{cases}2, & n=0 \\
1, & n \neq 0\end{cases}
\end{aligned}
$$




\subsubsection{Model Implementation and Results}

The perturbation analysis has been developed by using the numerical computation software MATLAB ${ }^{\circledR}$. The method, which provides the maximum frequency deviation between the case of the empty cavity and the cavity with the sample, can be used for materials with different complex permittivity.

Algorithm 8 computes the perturbation method in a $\mathrm{TE}_{m n p}$ rectangular cavity for a $R$ radius drop and gives the frequency deviation.

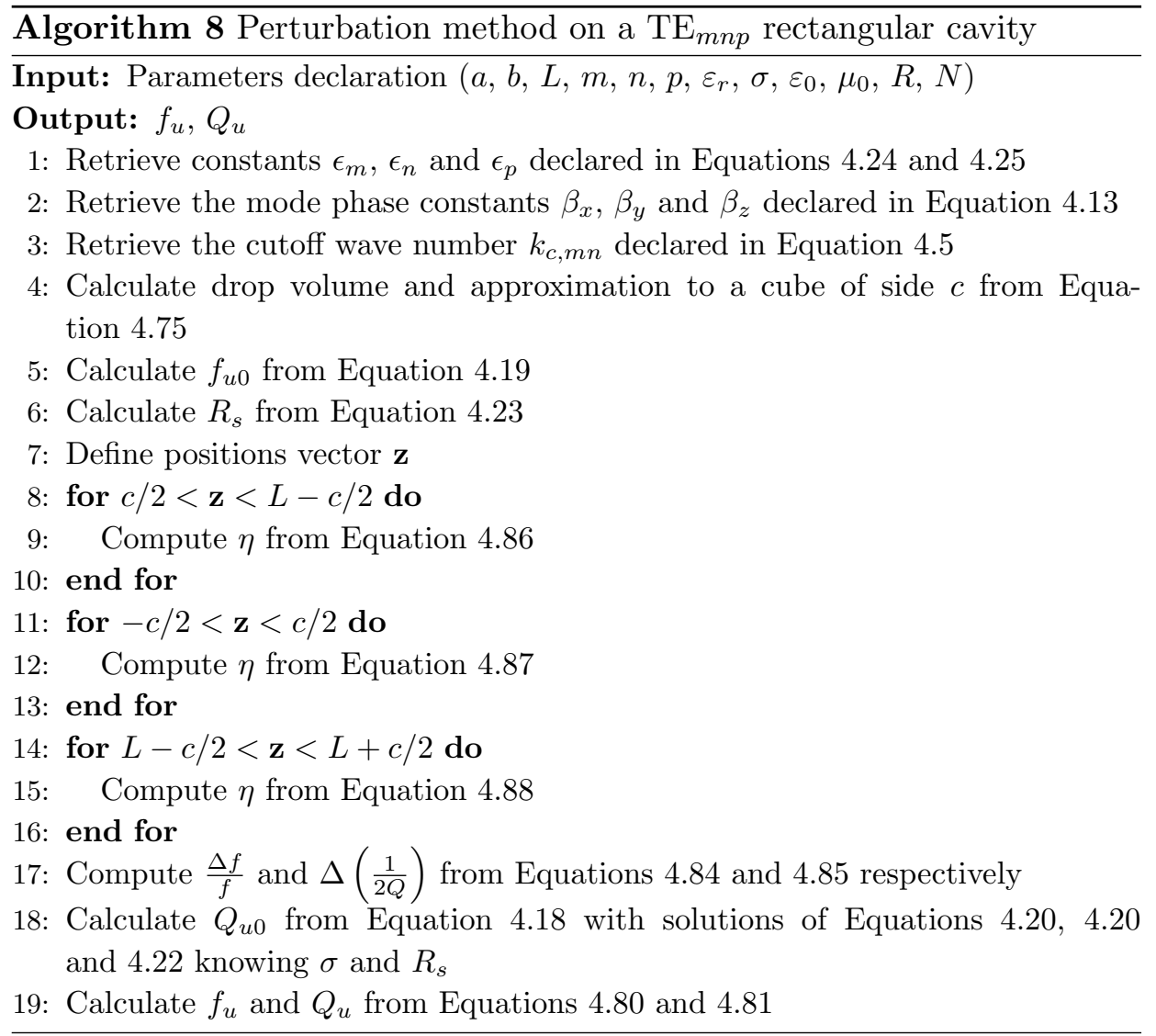

Figure 4.13 represents the maximum frequency deviation between the case of the empty cavity and the cavity with the sample for different values of the dielectric constant $\left(\varepsilon^{\prime}\right)$ and loss factor $\left(\varepsilon^{\prime \prime}\right)$. The values are calculated 


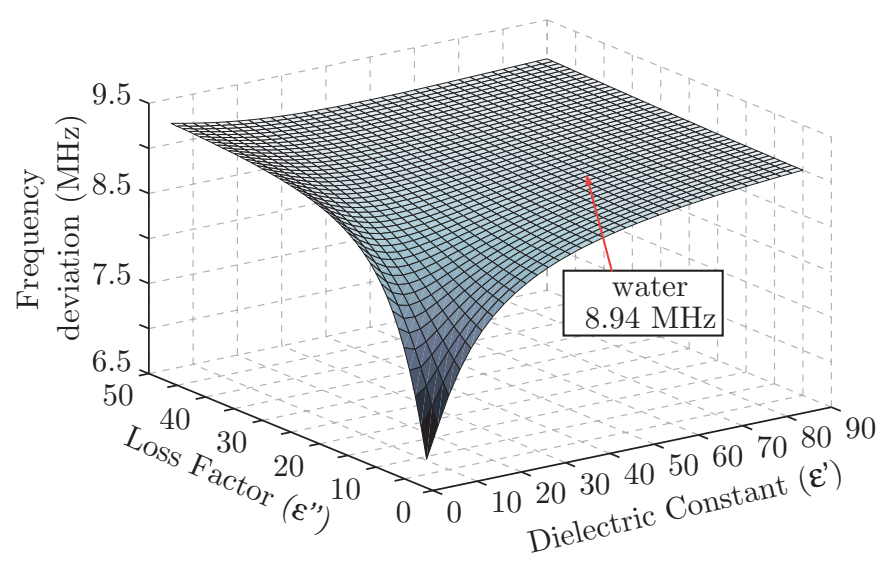

Figure 4.13. Calculated frequency deviation of a cavity while a $1.1 \mathrm{~mm}$ radius spherical sample falls through its central axis as function of $\varepsilon^{\prime}$ and $\varepsilon^{\prime \prime}$.

for the PINE rectangular cavity and for a $1.1 \mathrm{~mm}$ radius drop by entering the $\varepsilon_{r}$ values in Algorithm 8.

In the case of a water drop $\left(\varepsilon_{r}=55.8-j 33.5\right.$ [70] $)$ the maximal frequency deviation is $8.94 \mathrm{MHz}$. This point is indicated and labelled in Figure 4.13.

Figure 4.14 a) represents the calculated frequency deviation (Equation 4.80) produced by the same water drop when crossing the cavity as function of its position. Just like the frequency, the $Q$-factor of the cavity is affected by the drop perturbation. Figure $4.14 \mathrm{~b}$ ) shows $Q$ deviation as function of the drop position.

The frequency and $Q$-factor values provide the position dependent reflection parameter (Equation 4.89). Hence the real power sent to the cavity $\left(P_{i n}\right)$ can be calculated from Equation 4.90 knowing the frequency and the power of the generator $\left(f_{g e n}\right.$ and $P_{g e n}$ respectively) and the external quality factor $Q_{e}$. Algorithm 9, as an extension of Algorithm 8, develops this function.

Moreover the frequency values allow, from Equations 4.26 and 4.92, the extraction of the absorbed power by the walls of the cavity $\left(P_{0}\right)$ as well 

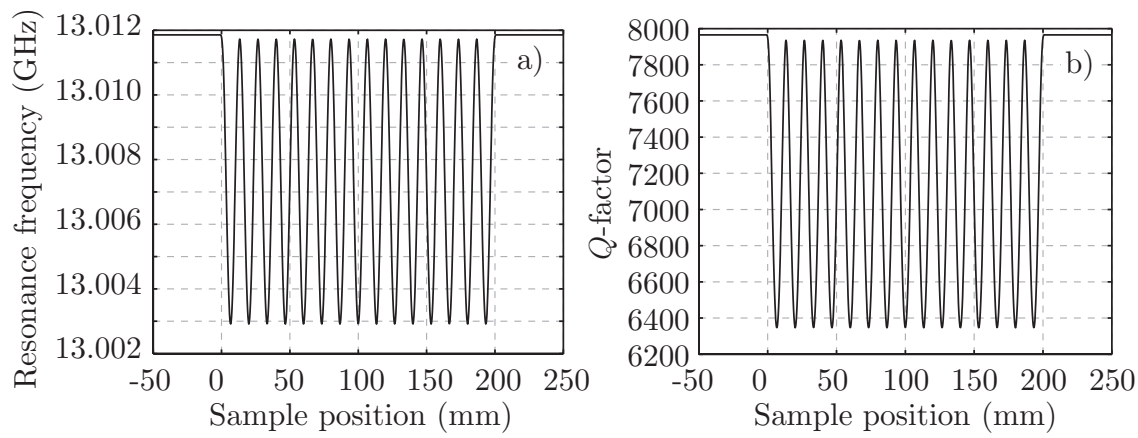

Figure 4.14. Calculated a) frequency and b) $Q$-factor deviation of a cavity while a water $1.1 \mathrm{~mm}$ radius spherical sample falls through its central axis as function of drop position.

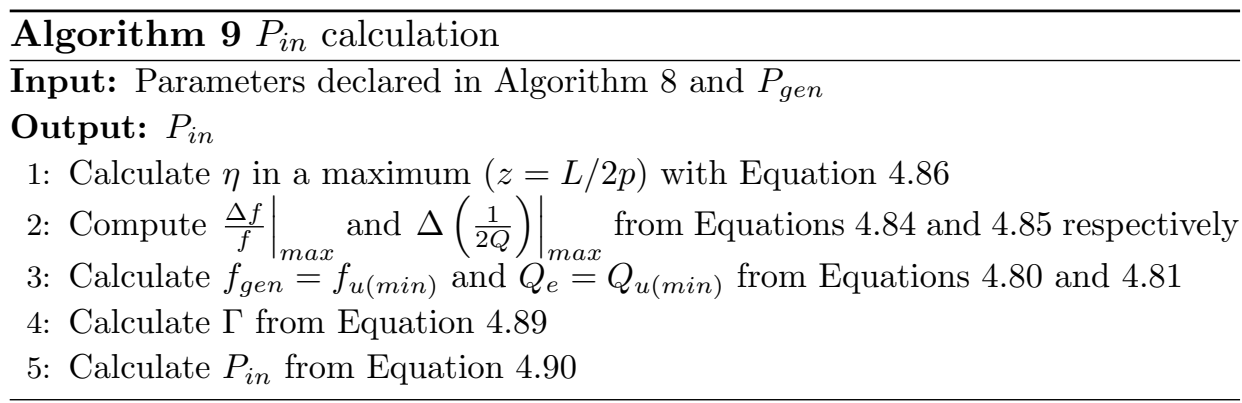

as the power dissipated in the drop $\left(P_{d}\right)$ respectively.

Algorithm 10 is a modified version of Algorithm 5 and computes the dissipated power in both the cavity walls and the drop using the values previously calculated in Algorithms 8 and 9.

The values of the drop dissipated power are represented in Figure 4.15 together with the input power $\left(P_{i n}\right)$ for the case of the previous described water drop.

As can be seen, the drop is not permanently dissipating significant power during its travel through the cavity. The maximal power is dissipated in the positions of maximum E-field. By applying the absorbed power to the thermal model through the heat generation rate $\left(Q_{\text {gen }}\right)$ which is solved by Equation 3.13, also expressed as $Q_{g e n}=P_{d} / v_{s}$, the maximal temperature 


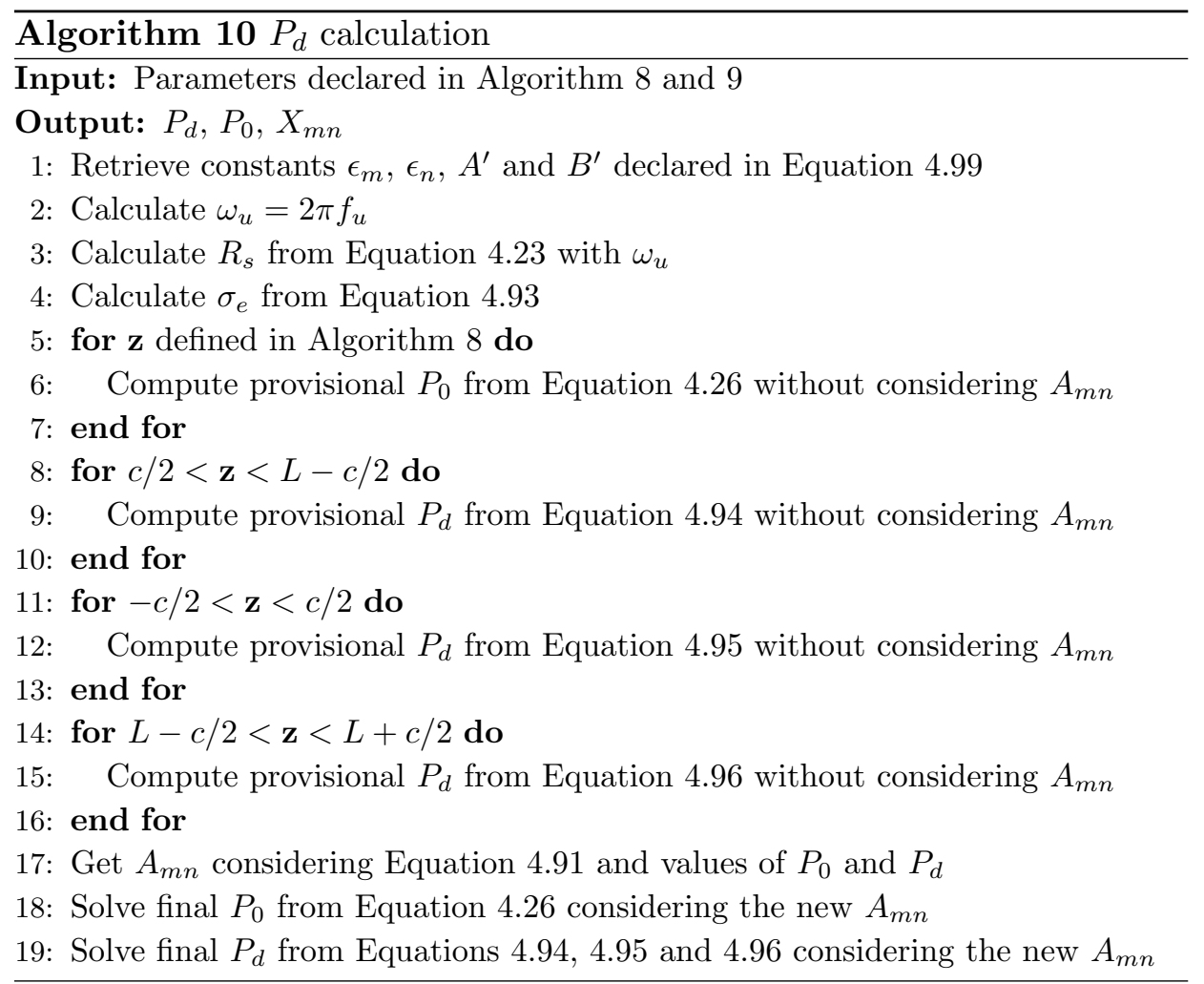




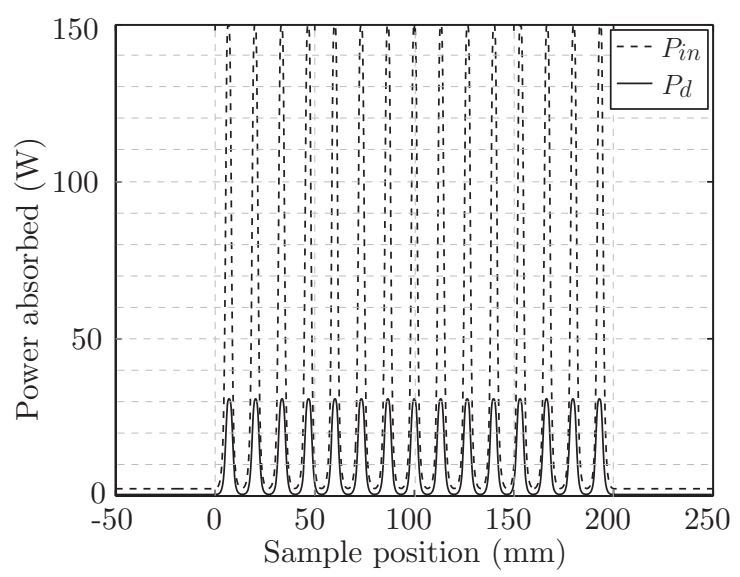

Figure 4.15. Calculated total absorbed power $P_{i n}$ and power absorbed by a $1.1 \mathrm{~mm}$ water drop $P_{d}$ while falling through the cavity as function of sample position for $P_{g e n}=150 \mathrm{~W}$.

of a drop, after crossing the cavity, is calculated for different values of dielectric constant $\varepsilon^{\prime}$ and loss factor $\varepsilon^{\prime \prime}$.

Algorithm 11 is an update of the thermal model presented in Algorithms 1, 2 and 3, where instead of fixing the $Q_{g e n}$ value, it is calculated from the values of $P_{d}$ inside the cavity. In this update the drop is also considered to have an acceleration while falling through the cavity. Therefore the convection heat transfer coefficient changes with position/time and is calculated by using expressions from Appendix A (given the kinematic viscosity $\nu_{a}$ and the specific heat capacity $c_{p_{a}}$ of air). 


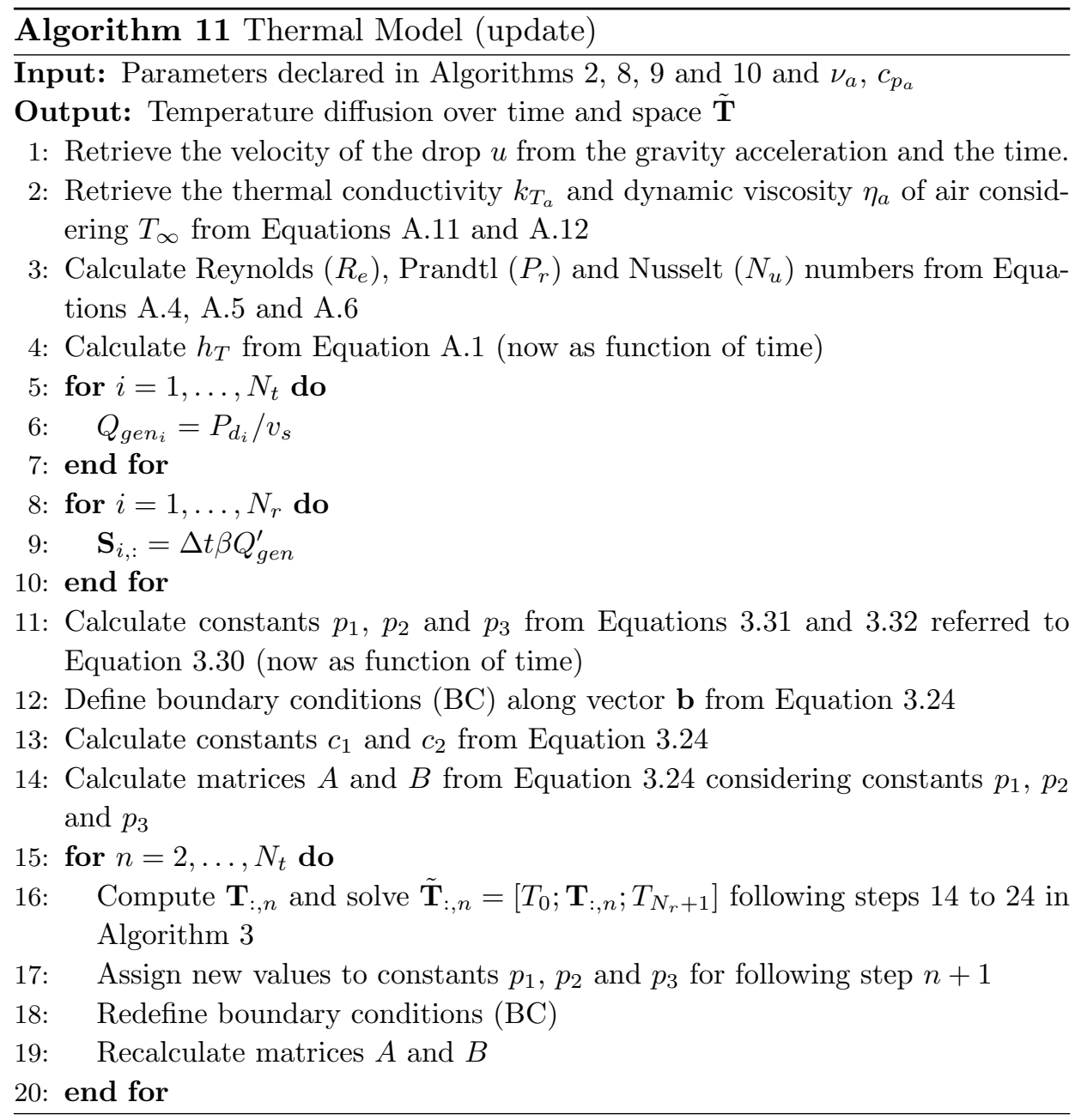




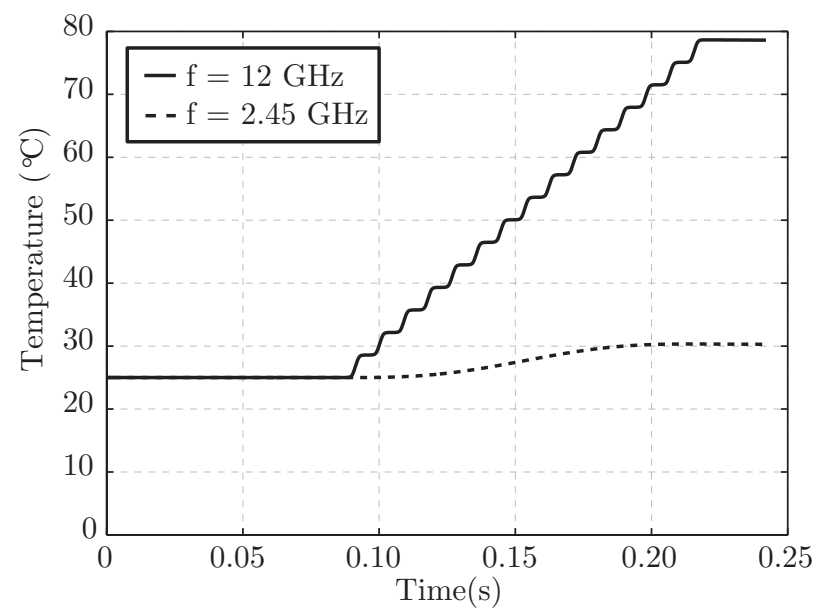

Figure 4.16. FDTD simulation of the heating rate for a 1.1 radius water drop using either 12 or $2.45 \mathrm{GHz}$ (incident power $150 \mathrm{~W})$.

Figure 4.16 displays the temperature evolution of a water drop ( $\rho=$ $1000 \mathrm{~kg} \cdot \mathrm{m}^{-3}, c_{p}=4190 \mathrm{~J} \cdot \mathrm{kg}^{-1} \cdot \mathrm{K}^{-1}, k_{T}=0.6 \mathrm{~W} \cdot \mathrm{m}^{-1} \cdot \mathrm{K}^{-1}$ ) during free fall from a drop generator $(t=0 \mathrm{~s})$ through a microwave rectangular cavity $(0.0869<t<0.2199 \mathrm{~s})$ as a function of residence time. Free convection of the air is considered as a main boundary condition $\left(h_{T}\right.$ calculated as explained in Algorithm 11). The water drop has $1.1 \mathrm{~mm}$ radius and takes $133 \mathrm{~ms}$ to cross the cavity, which length is $200 \mathrm{~mm}$ and which top entrance is located $37 \mathrm{~mm}$ below the drop generator. The cavity, simulated and applied to the perturbation and thermal models, is working with the $\mathrm{TE}_{10 N}$ mode and fed with a microwave power of 150 watts.

The Figure 4.16 shows the different temperature rates that can be achieved by designing the cavity at different frequencies. At X-band a water drop with an initial temperature of $25^{\circ} \mathrm{C}$ reaches the internal gelation temperature $\left(80 \pm 5{ }^{\circ} \mathrm{C}\right)$, which corroborates the experiments performed by other authors in $[13,14,42]$. However, in the S-band microwave frequency range at $2.45 \mathrm{GHz}$, the drop temperature hardly reaches $30{ }^{\circ} \mathrm{C}$ from the same initial temperature. Preliminary studies at this frequency reported high power rates e.g. $5000 \mathrm{~W}$ [15] or pre-heating by other sources [18] to 

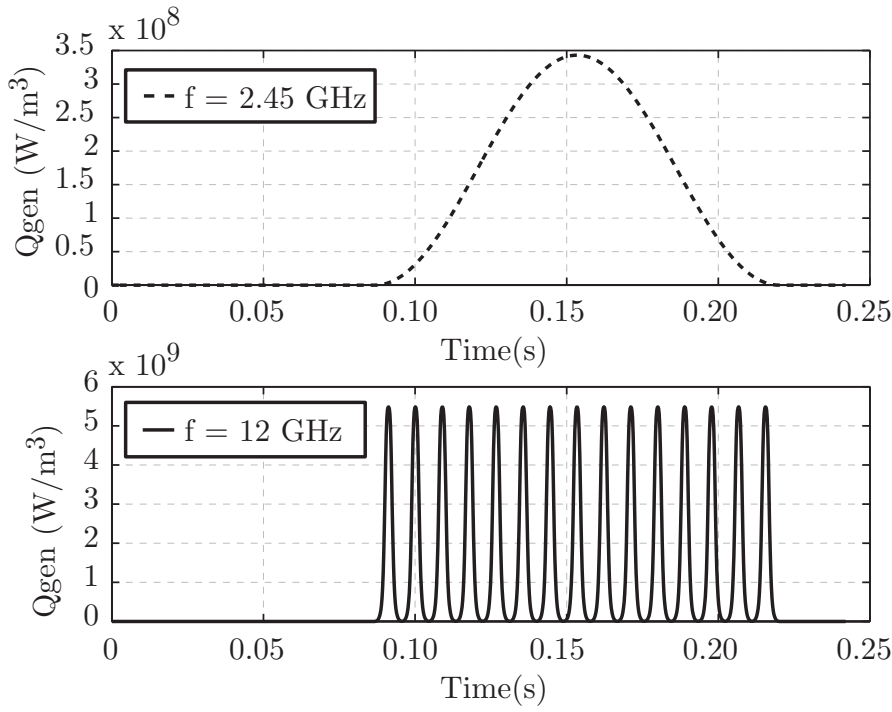

Figure 4.17. Qgen profile $(0.0869<t<0.2199 \mathrm{~s})$ applied to the FDTD thermal simulation of Figure 4.16 (incident power $150 \mathrm{~W}$, drop radius $1.1 \mathrm{~mm}$ ).

achieve the minimum temperature increase needed to trigger the gelation. The oscillations of the temperature evolution in Figure 4.16 correspond to the distribution of EM fields inside the microwave cavity which for X-band was $\mathrm{TE}_{10(15)}$ meanwhile for the S-band was $\mathrm{TE}_{101}$ for a total cavity length of approximately $200 \mathrm{~mm}$. Figure 4.17 shows the Qgen profile related with the generated fields inside the cavity for both cases.

The perturbation and thermal model, as it has been shown for water, can provide accurately the temperature diffusion according to the material properties for a selected frequency and power. Therefore, the dielectric properties of the feed solution for the production of fuel are also required in order to determine the frequency and power used for the MIG. A new experimental method, which is described in the next chapter, has been used to determined the dielectric properties of drops by means of a cylindrical microwave cavity.

Figure 4.18 offers a feasibility overview for heating a free falling ma- 


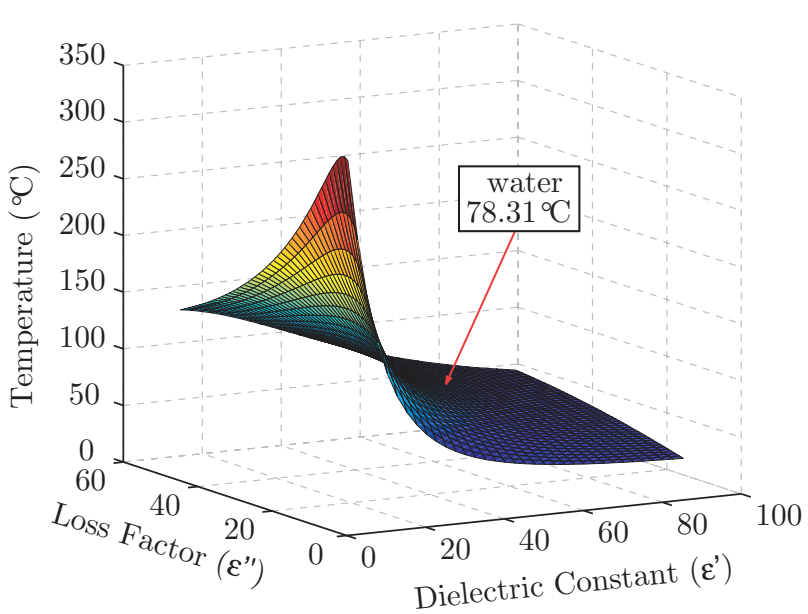

Figure 4.18. Calculated temperature of a $1.1 \mathrm{~mm}$ radius spherical sample after its fall through the cavity as function of $\varepsilon^{\prime}$ (from 5 to 90 ) and $\varepsilon^{\prime \prime}$ (from 5 to 50 ).

terial, in form of $1.1 \mathrm{~mm}$ radius drops, inside the PINE rectangular cavity (design parameters in Table 4.1). In case of water, the maximal reached temperature is $78.31^{\circ} \mathrm{C}$, a value that is indicated and labelled in the figure.

\subsection{Conclusions}

In this chapter, both rectangular and cylindrical cavities were presented. Their field equations and the calculation of their parameters, i.e. resonant frequency and quality factor, as well as the calculation of their energies and powers were developed for the case of empty cavities and cavities loaded with dielectrics.

The coupling system used to feed the cavities was shown to be crucial to transfer the power efficiently. Several software packages for the design of the cavities were commented. Specifically the numerical Matlab software was utilized to compute cavity models, with which the cavity parameters are solved and represented. 
In addition, the perturbation method was explained focused on the PINE project. The method was also processed with Matlab being used for connecting the cavities model and the thermal model presented in Chapter 3 . The results demonstrated the feasibility of heating falling drops inside the X-band rectangular cavity designed for the PINE project.

The main contributions of this chapter were published in [71, 72]. 



\section{Characterization of Materials}

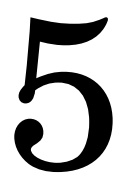

5.1 Introduction . . . . . . . . . . . . . . . . . . . 124

5.2 Dielectric Loss . . . . . . . . . . . . . . . . . . . . 124

5.3 Dielectric Measuring Techniques . . . . . . . . . . 128

5.4 New Characterization Method of Dielectrics . . . . . . 130

5.5 Perturbation and Thermal Models of Characterized Materials . . . . . . . . . . . . . . . . . 145

5.6 Conclusions . . . . . . . . . . . . . . . . . . . . . 148 



\section{Characterization of Materials}

The heating of A MATERIAL is governed by its dielectric properties. As the main intention of this thesis is to produce SP-fuel by Microwave Internal Gelation, it is important to know the dielectric properties of the feed solution that is going to be heated. Then, a better knowledge on the amount of power that is absorbed by the material will be provided. This chapter introduces an overview of the parameters that deal with the heating of a material by microwaves. In addition the different techniques used for the measurement of dielectric properties are presented. Because none of the conventional techniques was optimal for the project, a new dielectric properties characterization method is proposed and described considering the geometry and the influencing parameters of the samples that should be produced in the context of the PINE project. The experimental setup is presented and several experiments demonstrate the process feasibility. The measured dielectric properties are afterwards included in the perturbation and thermal models with the main intention of knowing the absorbed power by the material in form of drops and the reached temperature. 


\subsection{Introduction}

Electromagnetic waves at microwave and radio frequencies have been widely used for telecommunication. However, in the last half-century microwaves have also been used to interact with materials for other purposes like microwave diathermy (microwave heating).

The microwave energy penetrates in the whole volume of dielectric material and the electric field distribution along the material dictates its interaction quality and facilitates the information to recover the sample characteristics. Over the next sections the principle of the volumetric heating is explained as well as its main parameters.

\subsection{Dielectric Loss}

An insulating material can be heated by applying high frequency electromagnetic waves. The origin of this heating lies in the ability of the electric field to polarize the charges in the material and the inability of the polarization to follow the extremely rapid reversals of the electric field. The complex permittivity and complex polarizability of a material reflects its charge displacement dynamics. Both are function of excitation frequency and intensity [49].

\subsubsection{Polarization}

There are four basic mechanisms of polarization jointly or separately responsible in the heating of a material: space-charge, molecular, ionic and electronic (see Figure 5.1 [73]). The frequency determines the dominant mechanism [49].

- The space-charge polarization is known as an interfacial polarization and arises from charge build-up in interfaces between components in heterogeneous systems; this polarization dominates at lower frequencies, and it is very important in semiconductors.

- In the molecular polarization an external field aligns permanent dipoles 


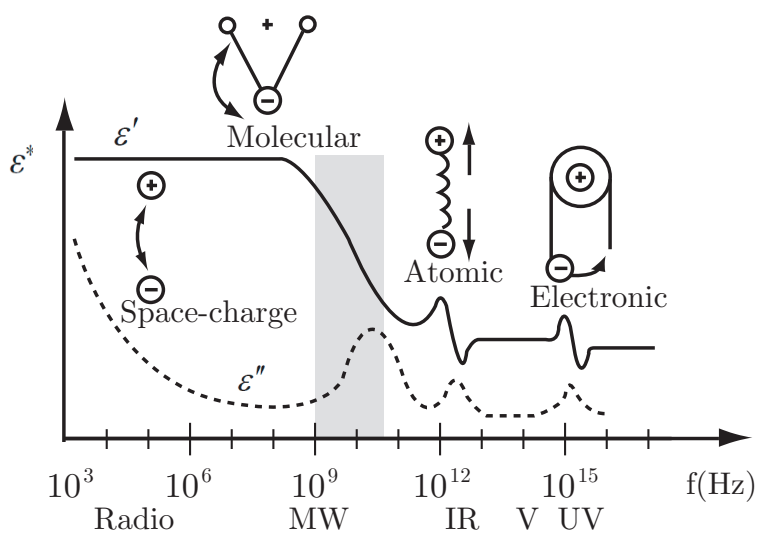

Figure 5.1. Frequency response of the permittivity in a material due to different polarization mechanisms. $\varepsilon^{\prime}$ represents the dielectric constant and $\varepsilon^{\prime \prime}$ the loss factor.

parallel to the field. In some materials it can be retained upon removal of the field due to the need for thermal activation of molecular rotation. The friction accompanying the orientation of the dipole will contribute to the dielectric losses. At microwave frequencies, the molecular polarization is the dominant mechanism because of the high number of aligned dipoles.

- The atomic polarization occurs due to the relative displacement of atomic nuclei because of the unequal charge distribution in the molecule formation. It is the dominant polarization at microwave frequencies for many dry solids.

- The electronic polarization is induced from the displacement of electrons around the atom core. This mechanism dominates at very high frequencies, where the ions cannot respond sufficiently fast to the field.

For a given dielectric material the sum of contributions for each mechanisms determines the net polarization.

$$
P=P_{s p-c h}+P_{m}+P_{a}+P_{e}
$$


where the subscripts refer to space-charge, molecular, atomic and electronic polarizations.

The molecular polarization is the dominant mechanism at microwaves frequencies for industrial heating [49]. The decrease of the effective polarization manifests itself as a decrease in the dielectric constant $\varepsilon^{\prime}$ and rise of the loss factor $\varepsilon^{\prime \prime}$ (see the shaded area in Figure 5.1). The energy is drawn from the system and is dissipated as heat into the material. A maximum energy is absorbed and $\varepsilon^{\prime \prime}$ comes to a resonant peak.

The equations that represent the microwave heating in a control volume should satisfy the first law of thermodynamics or the energy balance equation accounting changes in thermal energy storage. The temperature in a material can be determined from the Fourier energy balance equation which can be expressed as in equation $3.11[49,50]$.

\subsubsection{Complex Dielectric Constant}

When a field is applied to a dielectric the negative and positive charges are bounded in dipoles which are characterized by the average dipole moment $\vec{m}_{e l}$ as

$$
\vec{m}_{e l}=\sum_{i=1}^{N} q_{i} \vec{r}_{i}
$$

where $q_{i}$ and $\vec{r}_{i}$ are the charges and their separation respectively, and $N$ is the number of charges. The average dipole moment within a volume defines the polarization as:

$$
\vec{P}=\frac{d \vec{m}_{e l}}{d V}
$$

which can also be computed from the individual dipole moments:

$$
\vec{P}=\vec{m}_{e l} N_{D}
$$

where $N_{D}$ is the dipole density per volume unit.

The total charge of the system is given by the electric charge density vector $\vec{D}$ :

$$
\vec{D}=\varepsilon_{0} \vec{E}+\vec{P}
$$


In presence of a dielectric the density vector reads

$$
\vec{D}=\varepsilon_{s} \vec{E}
$$

where $\varepsilon_{s}$ is the static dielectric constant expressed as relative to the vacuum permittivity $\left(\varepsilon_{0}=8.854 \times 10^{-12} \mathrm{~F} \cdot \mathrm{m}^{-1}\right)$.

If the electric field of a system, where the dielectric is immersed, is dependent on time $\left(\vec{E}(t)=E_{0} \cos (\omega t)\right)$ with $\omega / 2 \pi$ being the frequency in $\mathrm{Hz}$, the density $\vec{D}$ will also be periodic with time, but not necessarily in phase with the field [74]:

$$
\vec{D}(t)=D_{0} \cos (\omega t-\phi)=D_{1} \cos (\omega t)+D_{2} \sin (\omega t)
$$

with (according to trigonometry):

$$
\begin{aligned}
& D_{1}=D_{0} \cos (\phi) \\
& D_{2}=D_{0} \sin (\phi)
\end{aligned}
$$

Then, from Equation 5.6 it can be seen that the dielectric is also dependent on the frequency:

$$
D_{1}=\varepsilon^{\prime}(\omega) E_{0}, D_{2}=\varepsilon^{\prime \prime}(\omega) E_{0}
$$

Consequently, in a real dielectric, the permittivity attains a complex form given by:

$$
\varepsilon^{*}(\omega)=\varepsilon^{\prime}(\omega)-j \varepsilon^{\prime \prime}(\omega)
$$

where the real part $\left(\varepsilon^{\prime}\right)$ is known as the dielectric constant and the imaginary part $\left(\varepsilon^{\prime \prime}\right)$ is the loss factor. When the frequency approaches zero the loss tangent approaches zero as well $\left(\varepsilon^{\prime \prime} \rightarrow 0\right)$ and the static case described in Equation 5.6 is accomplished with $\varepsilon^{\prime} \rightarrow \varepsilon_{s}$.

It can be foreseen that $\varepsilon^{\prime \prime}$ represents the energy loss in the dielectric due to the dipole lag $(\phi)$. This lag is due to the polarization, which cannot follow the rapid reversals of the field. Therefore the permittivity imaginary 
part includes both the conduction and the polarization losses, defining the effective loss factor as

$$
\varepsilon_{\mathrm{eff}}^{\prime \prime}(\omega)=\varepsilon_{s p-c h}^{\prime \prime}(\omega)+\varepsilon_{m}^{\prime \prime}(\omega)+\varepsilon_{a}^{\prime \prime}(\omega)+\varepsilon_{e}^{\prime \prime}(\omega)+\frac{\sigma}{\varepsilon_{0}} \omega
$$

where the subscripts refer to space-charge, molecular, atomic and electronic polarizations, and $\sigma$ is the metal electric conductivity.

\subsubsection{Volumetric Heating}

The design of a microwave applicator requires knowledge of the dielectric properties of the materials that are going to be processed.

In the interaction of a dielectric with microwave energy, the power is attenuated as the electromagnetic field penetrate the dielectric. This effect depends on the dielectric properties, specifically on the loss factor. The absorbed power in the dielectric reads:

$$
P_{d}=\omega \varepsilon_{0} \varepsilon_{\text {eff }}^{\prime \prime}\left|E_{r m s}\right|^{2} v_{s}
$$

which depends on the volume of the sample $v_{s}$. As seen in Chapter 3 the power per unit volume defines the volumetric heating through the heat generation rate concept $\left(Q_{g e n}=P_{d} / v_{s}\right)$, which is applied to the thermal model to know how the temperature is increased in a sample as function of the power and its dielectric properties.

\subsection{Dielectric Measuring Techniques}

The selection of an appropriate dielectric property measuring technique, also known as a Dielectric Spectroscopy (DES), depends on several aspects such as the kind of material, the sample preparation, the frequency band (narrow or broad), the measurement accuracy, the degree level of dielectric loss, etc.. A systematic technique classification is based on the frequency band: discrete frequency and broadband [75]. All DES techniques have strengths and weaknesses, and the researcher has to decide which accuracy he is aiming for. 
Discrete frequency methods include resonant methods over a few electromagnetic modes and they are used for accurate measurements on lowloss materials. In these resonant methods, the position and dimensions of the samples, the coupling or the dimensions of the cavity introduce uncertainties. Broadband methods transmit EM waves through a material while changing the frequency. In transmission lines measurements, for example, the air gaps also introduce uncertainties.

Some of the dielectric measuring techniques are described bellow.

\section{The Split Cylinder Resonator}

This resonator technique determines the permittivity of dielectric substrates from measurements of the resonance frequency and the quality factor, which is usually calculated from $Q=f_{0} / \Delta f$, with $f_{0}$ the empty cavity resonance and $\Delta f$ the frequency difference at $-3 \mathrm{~dB}$ of the resonance response.

The Split Cylinder Resonator is first measured empty, and then the sample, which is considered infinite in the radial direction, is inserted and the loaded resonance frequency and $Q$-factor is determined by computing the fields in the sample region. The permittivity is computed with a developed software from the measured $Q$-factor and resonant frequencies [76].

\section{Cylindrical Cavity}

The dielectric characterization of materials in a cylindrical cavity is done from the difference in frequencies and $Q$-factors between the case of the empty cavity and the cavity with a loaded sample.

A single-post coaxial reentrant cavity with a partially dielectric filled gap is described in [77]. Both loss and high loss dielectric materials are introduced in the cavity and their permittivity is computed with a developed software based on Generalized Scattering (GSM) or Admittance (GAM) Matrices [78, 79] considering a previous calibration of the cavity for recovering the unloaded frequencies [58]. 


\section{Open-ended Coaxial Line}

The open-ended coaxial line technique is used principally in liquids and semiliquid materials. The probe, with which the dielectric samples are analysed, consists on a truncated coaxial cable composed by an internal conductor insulated with a dielectric from the external conductor [80].

The probe is immersed in a test material, where the electromagnetic signal coming from a Vector Network Analyser (VNA) generates an electric field between the conductors over the dielectric medium, which is formed by the dielectric of the probe and the dielectric of the tested material. The input reflection coefficient is then collected and used together with the original signal to recover the material dielectric properties $\left(\varepsilon^{\prime}\right.$ and $\left.\varepsilon^{\prime \prime}\right)$ from the attenuation and the phase constants, i.e. from the propagation constant.

This technique is able to determine the dielectric properties over a wide frequency range $(100 \mathrm{MHz}-20 \mathrm{GHz})$ in a very short time, around $3-4$ seconds, which is an advantage for the identification of changes in molecular structures or dynamic processes and reactions like gelation. However, these chemical reactions could corrode the probe invalidating the dielectric properties measurements.

Over the next section a new narrow band technique to accurately measure the feed solution used for the SP-fuel production in the PINE project is presented.

\subsection{New Characterization Method of Dielectrics}

In this section the method for the dielectric property evaluation of a material in form of drops is introduced. First the experimental setup for the measurements of the resonant frequency is described. Afterwards the procedure of the method in order to obtain satisfactory results is explained. Finally the experimental measurements and the results are presented and discussed. 


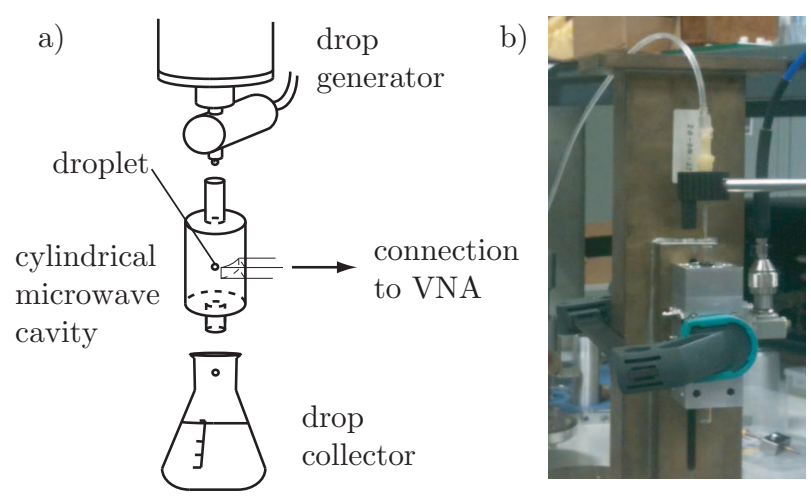

Figure 5.2. Experimental setup for the measurement of dielectric properties of a single drop: a) schema and b) photograph. Cavity: $50 \mathrm{~mm}$ length, $9 \mathrm{~mm}$ radius, $4.05 \mathrm{~mm}$ top and bottom holes radius. Drop $2.9 \mathrm{~mm}$ diameter. Temperature $25{ }^{\circ} \mathrm{C}$.

\subsubsection{Experimental Setup}

Figure 5.2 represents a schematic and photographic view of the dielectric measurements setup. A microwave cavity of cylindrical cross section has been designed, operating in the mode $\mathrm{TM}_{010}$ around the frequency of $10 \mathrm{GHz}$ (50 $\mathrm{mm}$ length, $9 \mathrm{~mm}$ radius, $4.05 \mathrm{~mm}$ top and bottom holes radius).

The length of the cavity has been designed considering two main principles: on one hand, the maximum length i.e. the longest residence time of the drop inside the cavity has been selected; on the other hand the operating resonant mode $\left(\mathrm{TM}_{010}\right)$ remains at the lowest frequency avoiding the confusion with high order modes in the same frequency band.

\section{Cavity Coupling}

The microwave cavity is connected, either through a magnetic loop or an aperture in a rectangular waveguide, to a Vector Network Analyzer (VNA). The magnetic loop was used in a previous study performed with water drops in order to prove the method feasibility. In the later work the use of an aperture enables the measurement of a liquid permittivity while changing 


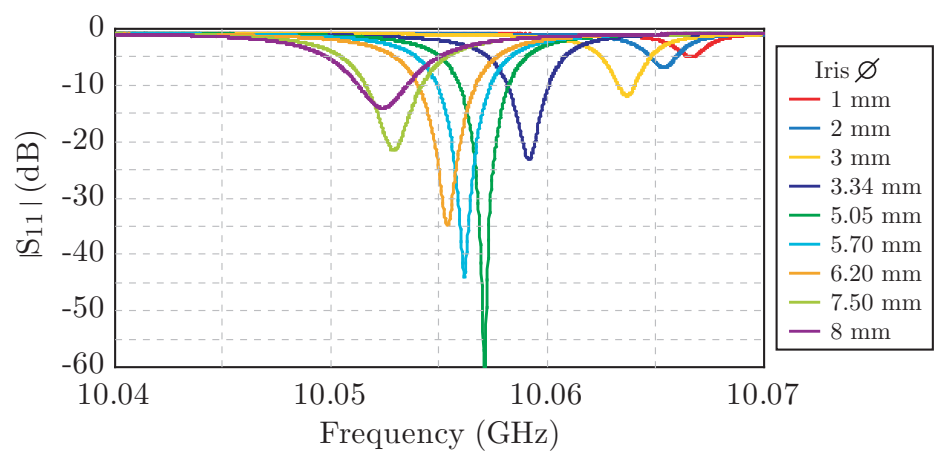

Figure 5.3. Coupling measurements: $\left|S_{11}\right|$ modulus as function of iris diameter in mm. Smaller to bigger iris dimensions are presented from right to left.

its temperature. In this case the feed solution described in Section 2.4.3 is refrigerated to avoid a premature gelation and this cooling is also applied to the cavity.

To evaluate the coupling of the cavity, several measurements of the input return losses were carried out while enlarging the diameter of the aperture, i.e. iris, by means of a VNA. These measurements allow the recovering of the natural unloaded resonant frequency and $Q$-factor of the cavity by using the model described in Section 4.4.1 [58].

Figures 5.3 and 5.4 show the modulus and phase representation of the input return losses respectively while the iris was enlarged in the cylindrical cavity with a quartz tube inside. The measurement was also carried out in the cavity without quartz.

The measured unloaded resonance frequencies were $12.8267 \mathrm{GHz}$ for the empty cavity and $10.0914 \mathrm{GHz}$ for the cavity with quartz, and the quality factors were 7955 and 5599 respectively.

The final iris has a diameter of $8 \mathrm{~mm}$ and, as seen in Figure 5.4, the representation in the Smith chart (larger circumference) is overcoupled. This aperture was chosen to compensate for the undercoupling effect when samples pass through the cavity. Therefore the sample will compensate the overcoupling to work in an ideal critical situation (circumference passing 
through the center of the Smith chart) and to ensure an efficient transmission of the energy.

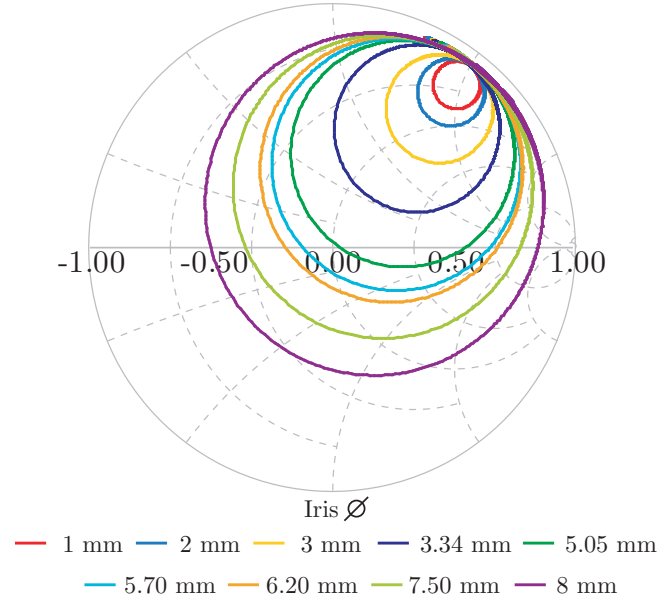

Figure 5.4. Coupling measurements: $\left|S_{11}\right|$ phase as function of iris diameter in $\mathrm{mm}$. The larger the iris dimensions, the larger the coupling (circumference diameter).

\section{Cavity working mode}

The mode $\mathrm{TM}_{010}$ has been selected for this application because of its maximum interaction with the spheres due to the constant electric field pattern in height, concentrated along the axis of the cavity. Figure 5.5 displays the magnitude of the Electric Field distribution in the cavity determined with the aid of the 3D EM Simulator QW3D [61].

Ideally the $\mathrm{TM}_{010}$ mode should keep the electric field constant along the cavity height. However, the openings at the top and the bottom of the cavity perturb the nominal electric field reducing the intensity at the borders and concentrating the maximum at the center of the cavity, where the interaction of microwaves with the drops is ideal.

\section{Feed solution}

The solution falls from the drop generator through holes at the top and the bottom of the cavity. The nozzle is adjusted to produce $1.47 \mathrm{~mm}$ radius 


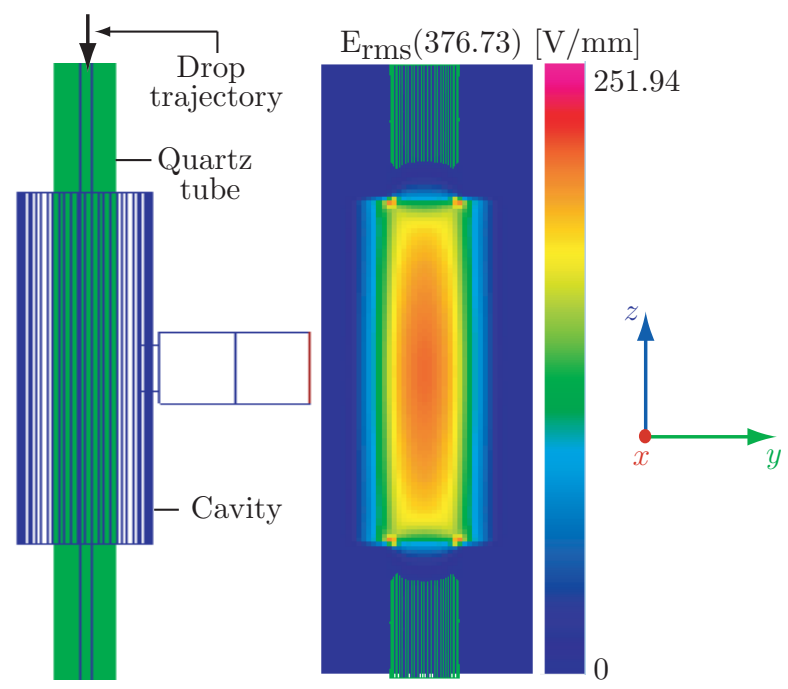

Figure 5.5. QW3D simulation (frontal and transversal view) of the magnitude of the E-Field distribution in a $\mathrm{TM}_{010}$ microwave cylindrical cavity at $10 \mathrm{GHz}$.

water drops, $1.12 \mathrm{~mm}$ radius methanol drops and $1.3 \mathrm{~mm}$ radius HMTA cerium based drops. A quartz tube (4 mm outer and $2.9 \mathrm{~mm}$ inner radius) is placed inside the microwave cavity along the axis to protect the walls from contamination from the droplets passing through.

\subsubsection{Drop Measurement}

The resonances measurement in microwave cavities with static samples can be carried out in a straightforward manner with an automatic VNA. However the conditions are different for a single sphere in motion e.g. a drop falling by gravity. Because the residence time of a single drop crossing the cavity is very short e.g. $52 \mathrm{~ms}$ being much faster than the analyzing VNA device response ( $\approx 20 \mathrm{~ms}, 100$ points, Rohde\&Schwarz ZVA 50 ), the frequency response cannot easily be captured. Therefore a new procedure is needed.

The method for the free falling drop consists of the excitation of the cylindrical cavity with a fixed frequency while the input return loss parame- 


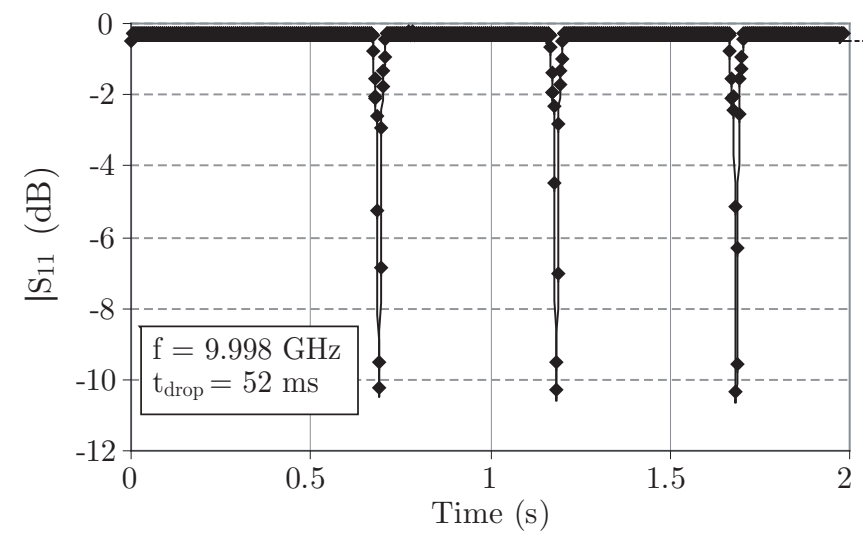

Figure 5.6. $\mathrm{S}_{11}$ measurement of the $\mathrm{TM}_{010}$ cylindrical microwave cavity at a frequency of $9.998 \mathrm{GHz}$ while 3 consecutive drops are passing through.

ter ( $\mathrm{S}_{11}$ amplitude and phase) is recorded with the aid of a VNA. The fixed frequency is varied stepwise while the water drops are falling by gravity along the axis of the cavity. Measurements are repeated over a frequency range large enough to recover the $S_{11}$ response and evaluate the resonant frequency displacement with regard to the other cases (i.e. empty cavity that can be straightforward measured with the VNA).

Figure 5.6 shows the measurements of the cavity with droplets at a single frequency as a function of time. Each pulse represents one drop through the cavity. The registered pulse length corresponds to the time that the drop takes to cross the cylindrical cavity. In the current study the time is $52 \mathrm{~ms}$.

If the pulses are enlarged, as seen in figure 5.7, it is possible to appreciate that the shape of the pulses changes depending on the selected frequency, which can be the resonance of the empty cavity, the resonance of the cavity with the drop or an intermediate step between both cases.

At the resonant frequency of the empty cavity, $10.054 \mathrm{GHz}$ (figure 5.7 a)), it is possible to observe how the $\mathrm{S}_{11}$ response is detuned and therefore presents a minimum value during the time that the drop remains inside the 

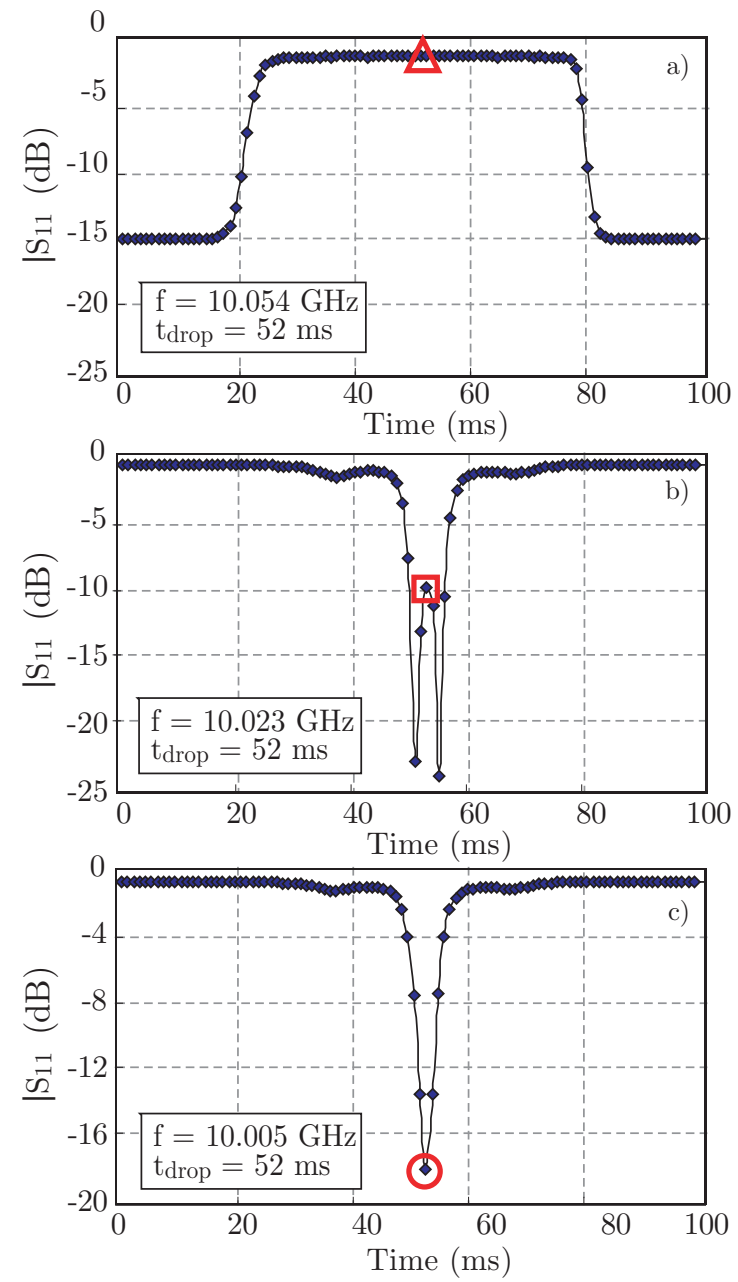

Figure 5.7. $\mathrm{S}_{11}$ measurement of the $\mathrm{TM}_{010}$ cylindrical microwave cavity for a single drop at: a) the resonant frequency of the empty cavity $10.054 \mathrm{GHz}$, b) a transient frequency $10.023 \mathrm{GHz}$ between resonances, and c) the resonant frequency of the cavity with the drop $10.005 \mathrm{GHz}$.

cavity. Using this value as starting point the frequency is decreased until the complete resonant frequency of the cavity with the drop is recovered. At $10.023 \mathrm{GHz}$ (figure $5.7 \mathrm{~b}$ )) the $\mathrm{S}_{11}$ parameter has a symmetrical response 
with respect to the middle point of the cavity. The return losses value is now reduced at the borders because this frequency is crossing the transient below the empty cavity resonant frequency. However this point is entering the transient above the resonant frequency of the cavity with a drop in the middle and therefore the value at the center is increased. At $10.005 \mathrm{GHz}$ (figure $5.7 \mathrm{c}$ )) the $\mathrm{S}_{11}$ parameter is maximal when the drop is at the center of the cavity i.e. this frequency corresponds to the resonant frequency of the cavity with the drop centered inside.

The middle point of these pulses (marked in figure 5.7, and later in figures 5.11 and 5.12 with a red triangle, square and circle for the $10.054 \mathrm{GHz}$, 10.023 GHz and $10.005 \mathrm{GHz}$ respectively), corresponds to the case when the drop is at the cavity center. This point is elected to reconstruct the frequency response.

Figure 5.8 displays an overview of the $\mathrm{S}_{11}$ amplitude measurements

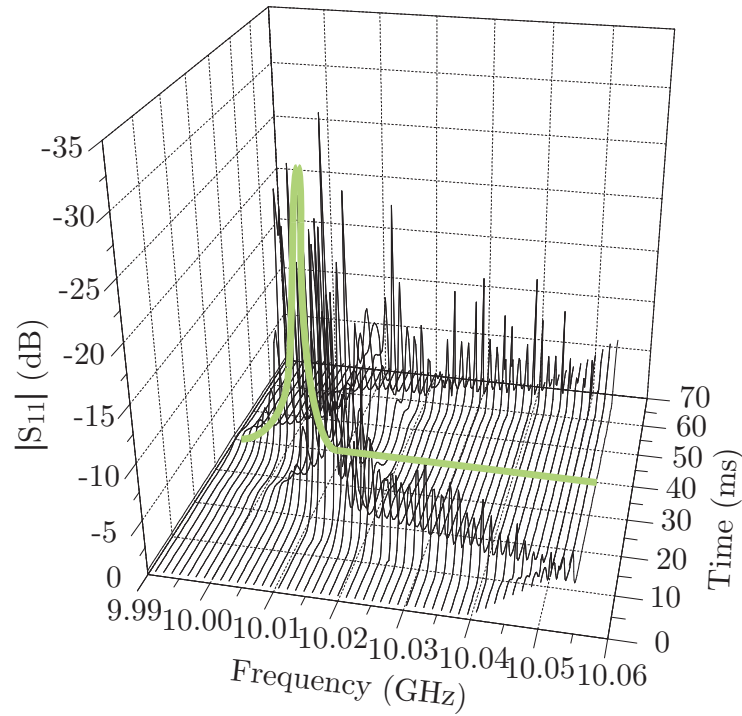

Figure 5.8. $\mathrm{S}_{11}$ measurement of the cylindrical microwave cavity as a function of the residence time, over the frequency range of the cylindrical cavity $\mathrm{TM}_{010}$ mode, while consecutive $1.47 \mathrm{~mm}$ radius water drops are passing through. 
over time for all the frequency steps used in this drop measurement for the frequency band of the selected mode. It is possible to observe the transient of the pulse shape from the empty (highest frequency) to the drop center loaded cavity case as a function of frequency. Cutting the representation along the $40 \mathrm{~ms}$ point on the time axes also results in the full frequency response when the drop is in the center of the cavity, i.e. in case of maximal deviation of frequency with respect to the case of the empty cavity (see approximation represented with a light green line).

\subsubsection{Drop Simulation}

The software CST Microwave Studio [62] is used with the main intention to simulate the behaviour of a drop while passing through the cavity. The cylindrical cavity used during the experiments is designed with the same characteristics than those described in Section 5.4.1 (Figure 5.9).

The mesh used for the simulation is adapted to the zones being analysed. In Figure 5.9, the finest mesh can be identified around the drop.

A drop is moved in $1 \mathrm{~mm}$ steps from $0 \mathrm{~mm}$ (cavity entrance) to $50 \mathrm{~mm}$

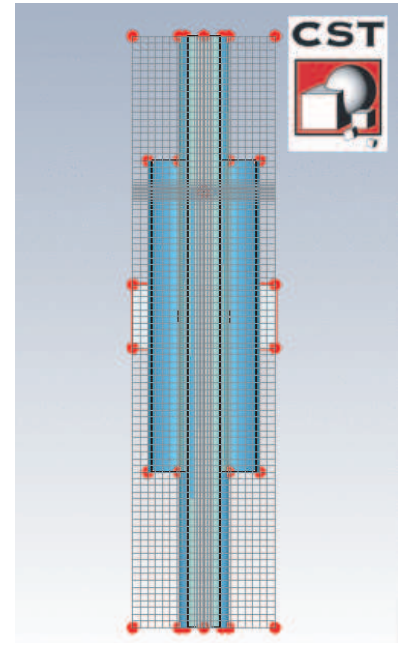

Figure 5.9. CST simulation of the cylindrical cavity with a $1.45 \mathrm{~mm}$ radius drop passing through its axis. 


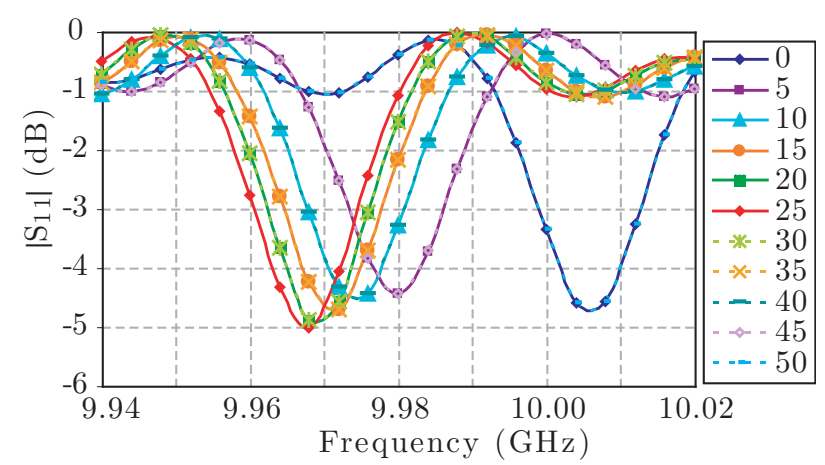

Figure 5.10. CST simulation of the resonant frequency shift while a $1.45 \mathrm{~mm}$ radius drop is passing through a cylindrical microwave cavity.

(cavity exit). The final result is in agreement with the one represented in figure 5.8 (but shifted in frequency). In figure 5.10 the input return losses are represented for positions taken every $5 \mathrm{~mm}$ steps.

The frequency response is symmetrical with respect to the center of the cavity: for a droplet travelling from the top to the center of the cavity the resonant frequency is shifted from a higher to a lower value; for a droplet travelling from the center to the bottom of the cavity the positions of the resonances are repeated in the contrary direction, i.e. from a lower to a higher frequency. The symmetry is in position but not in time because of the free fall of the drops being accelerated in the real case.

This representation gives further information about the interaction of the drop with respect to the cavity. Most of the resonances are close in frequency $( \pm 10 \mathrm{MHz})$ as the drop is close to the center. Any frequency of this range, and not only the frequency of the maximal excursion (center of the cavity), can be set in the frequency generator for the heating of drops in a microwave cavity to provide a maximal interaction with the fields.

\subsubsection{Experimental Measurements and Results}

Figure 5.11 shows the Smith chart representation of the frequency response of the input return losses $\left(\mathrm{S}_{11}\right)$ of the microwave cavity with different ma- 


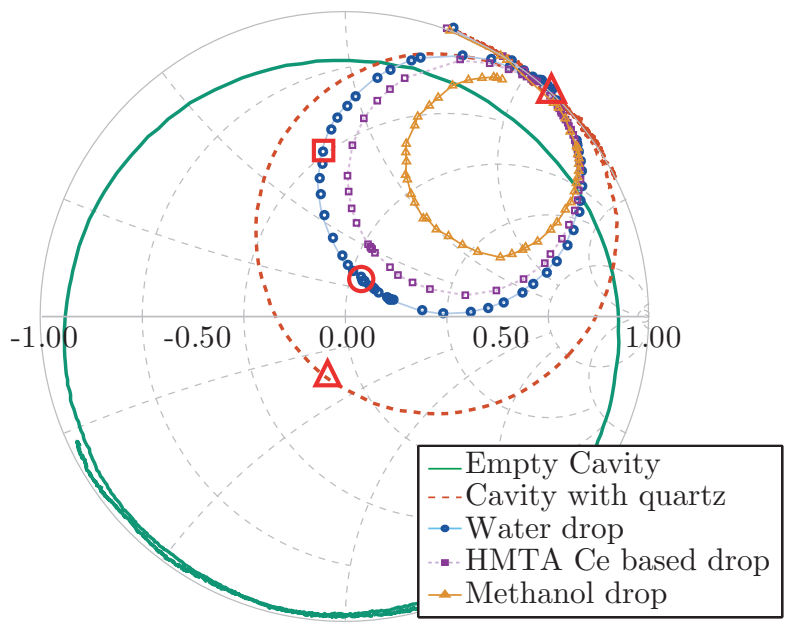

Figure 5.11. Smith chart representation of the reflexion parameter $S_{11}$. Influence of a drop presence in the center of the cavity on the resonance $\mathrm{TM}_{010}$ mode for different materials. Red marks (circle, square and triangle) correspond to the ones represented in figures 5.7 and 5.12. The triangle is double represented to indicate the relationship with the resonance of the cavity with quartz without drop.

terials at its center reconstructed stepwise with the procedure mentioned above (Section 5.4.2) for the resonant mode $\mathrm{TM}_{010}$. The $\mathrm{S}_{11}$ is measured through an aperture in a rectangular waveguide. The figure also shows the frequency response of the empty cavity and quartz loaded cavity measured conventionally with the VNA.

Since the representation of the $S_{11}$ parameter around the resonant frequency in this chart is a circle, this complex representation (with real and imaginary part) proved to be very useful to identify the correct module amplitude of each frequency measurement point of the cavity with different drops. It is possible to perceive that the middle points selected in figure 5.7 to reconstruct the resonant frequency (red signs) perfectly match the same single circle in the Smith chart. The red circle mark at $10.005 \mathrm{GHz}$ has the maximal excursion (diameter) in the representation of the $\mathrm{S}_{11}$ parameter and guarantees the most suitable resonant frequency value. 


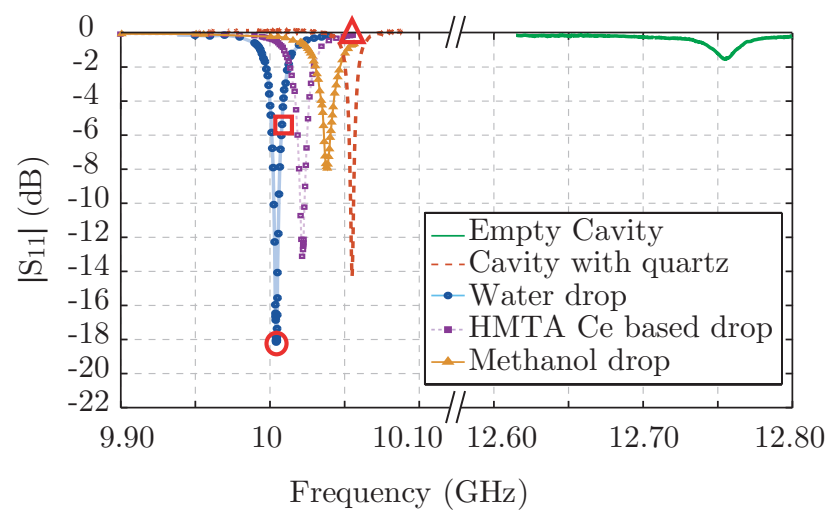

Figure 5.12. Magnitude of the $\mathrm{S}_{11}$. Influence of a drop presence in the center of the cavity on the resonance $\mathrm{TM}_{010}$ mode for different materials. Red marks (circle, square and triangle) correspond to the ones represented in figures 5.7 and 5.11 .

Figure 5.12 represents the frequency response of the input return losses $\left(\mathrm{S}_{11}\right)$ magnitude of the microwave cavity. Drop measurements are represented together with the measurement of the empty cavity and the cavity with quartz tube, for the resonant mode $\mathrm{TM}_{010}$ in the frequency range $(9.9-12.8 \mathrm{GHz})$. A shift of the resonant frequency for this mode can be seen when a drop is inside the cavity compared to the empty cavity with the quartz tube.

Table 5.1 summarizes the numerical values of the resonant frequencies $\left(f_{r}\right)$ and $Q$-factors for the measurements illustrated in figures 5.11 and 5.12. The calibration parameters of the cavity with the quartz tube, according to the procedure described in [58], were applied to the measurements with drops to determine the unloaded resonant frequencies. It also shows the permittivity values of the samples, obtained with the method described below.

\section{Dielectric Properties}

The procedure to determine the permittivity of the drops is based on the Circuit Analysis Method. This method consists of a segmentation of large electromagnetic problems (large structures) into smaller ones. Each simple 


\begin{tabular}{|c|c|c|c|c|}
\hline & $f_{r}(\mathbf{G H z})$ & $Q$-factor $(-)$ & $\varepsilon^{\prime}(-)$ & $\varepsilon^{\prime \prime} / \sigma(-)$ \\
\hline Empty Cavity & 12.7556 & 7532.26 & -- & $2.069 e 7 \mathrm{~S} \cdot \mathrm{m}^{-1}$ \\
\hline Cavity with quartz tube & 10.0551 & 6817.01 & 3.67 & 0.0004 \\
\hline $\begin{array}{l}\text { Cavity with quartz and } \\
\text { water single drop } \\
\text { (1.47 mm radius) }\end{array}$ & 10.0048 & 1597.05 & $\begin{array}{c}65.63 \pm 3.35 \\
(63.8[70])^{*}\end{array}$ & $\begin{array}{c}29.51 \pm 2.45 \\
(28.7[70])^{*}\end{array}$ \\
\hline $\begin{array}{l}\text { Cavity with quartz and } \\
\text { methanol single drop } \\
\text { (1.12 mm radius) }\end{array}$ & 10.0381 & 1038.05 & $\begin{array}{c}10 \pm 0.36 \\
(7.94[81])^{*}\end{array}$ & $\begin{array}{l}8.46 \pm 0.50 \\
(8.22[81])^{*}\end{array}$ \\
\hline $\begin{array}{l}\text { Cavity with quartz and } \\
\text { HMTA cerium based single } \\
\text { drop ( } 1.30 \mathrm{~mm} \text { radius })\end{array}$ & 10.0222 & 1331.77 & $\begin{array}{c}34 \pm 2.83 \\
(30.56[82])^{*}\end{array}$ & $\begin{array}{c}28.3 \pm 2.56 \\
(30.44[82])^{*}\end{array}$ \\
\hline
\end{tabular}

Table 5.1. Resonant frequencies, $Q$-factors and dielectric properties recovered from the measurements.

structure is characterized with the Generalized Admittance Matrix (GAM) and then all the simple structures are joined together, applying the boundary conditions, to analyse the whole complex structure [78, 79].

Figures 5.13, 5.14 and 5.15 show the dielectric constant and loss factor as a function of the assumed liquid drop radius. The radius is fixed by the drop generator which provides drops from $0.4 \mathrm{~mm}$ to $3 \mathrm{~mm}$ by changing its nozzle size. The permittivity values are calculated as a function of the drop radius with the Circuit Method described above, for the resonant frequency and $Q$-factor of the cavity in each situation (Table 5.1). The method provides the permittivity value independently of the radius dimension. Then, the relationship can also be used to fix the drop dimensions from a sample of known dielectric properties (such as water) and also indicates how the impact of the uncertainties in the drop dimensions can affect the permittivity determination.

The uncertainties represented in the Figures 5.13, 5.14 and 5.15 have 


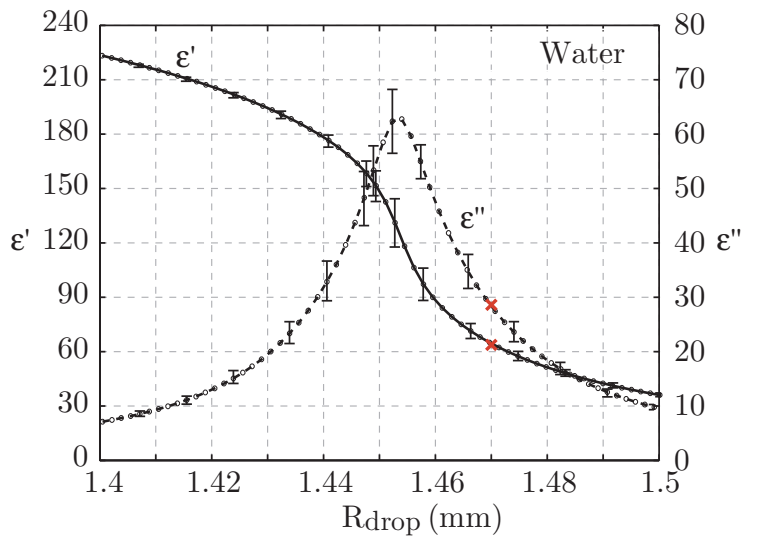

Figure 5.13. Calculated apparent dielectric properties as a function of assumed water drop radius.

been calculated, following the method described in [78, 83, 84], considering a $\pm 0.1 \mathrm{MHz}$ frequency, $\pm 5 Q$-factor, $\pm 0.1 \mu \mathrm{m}$ cavity radius, $\pm 0.1 \mu \mathrm{m}$ quartz radius and $\pm 1 \mu \mathrm{m}$ cavity height variations. The frequency variation is based on the frequency generator uncertainty and $Q$-factor variation has been empirically estimated from the deviation of the $Q$-factor value after several measurements with the system. Finally, the variations used for the cavity and quartz dimensions uncertainties are the ones provided by the manufacturers.

Table 5.1 shows the values of dielectric properties determined with this procedure for water (1.47 $\mathrm{mm}$ radius), methanol (1.12 $\mathrm{mm}$ radius) and HMTA cerium based solution (1.30 mm radius). For the cases of water and methanol, the calculated results are compared with published values measured with the open-ended coaxial method [70, 81].

As the measured values are in the range of the dielectric properties for water and methanol at these frequencies for the radius given by the drop generator, the method is validated and allows the measurement of new liquids. Then the dielectric properties of the HMTA cerium based solution used in the MIG process are finally determined. This dielectric properties were also validated by measuring them with the open-ended coaxial 


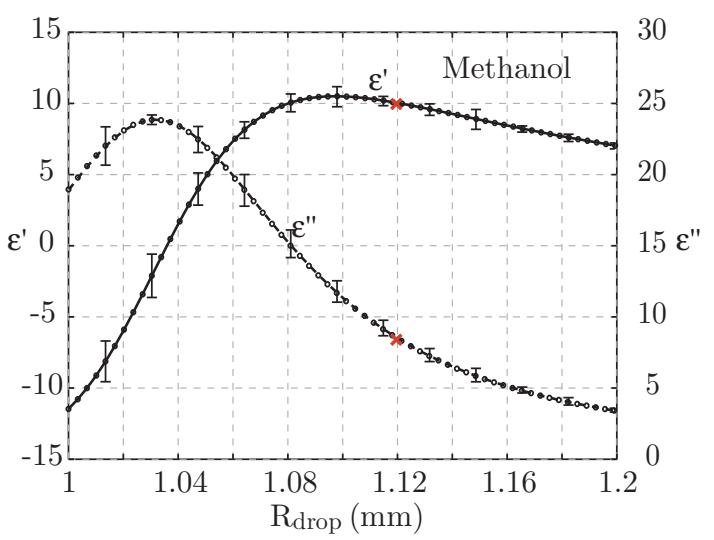

Figure 5.14. Calculated apparent dielectric properties as a function of assumed methanol drop radius.

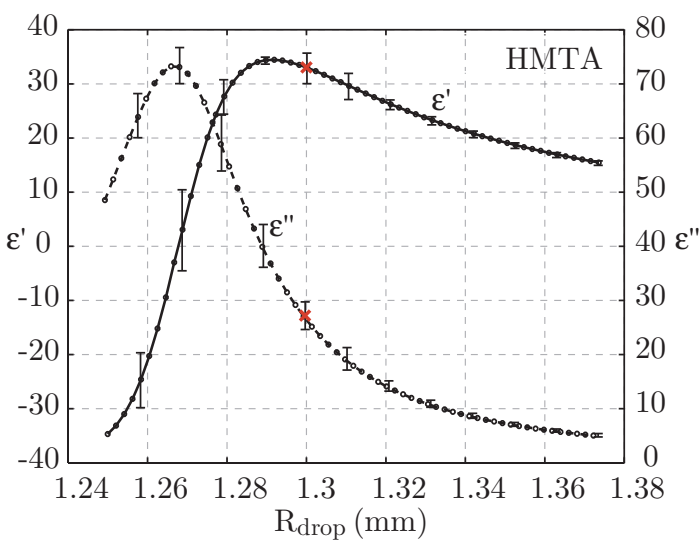

Figure 5.15. Calculated apparent dielectric properties as a function of assumed methanol drop radius.

method (work performed by a Master Student in the frame of the PINE project [82]). During the measurement, the chemical reaction manifested a corrosion of the probe, which states the decision of developing the new measurement method presented here. 


\subsection{Perturbation and Thermal Models of Characteri- zed Materials}

The measured permittivity of the HMTA cerium based solution $\left(\varepsilon_{r}=\right.$ $34-28.3 j$ ) is used as input parameter in Algorithm 8 (see Page 110) to recover analytically the deviation of the frequency and Quality factor when a drop of this solution falls down through a rectangular cavity with the main intention of triggering the gelation. Figure 5.16 shows the variation of these parameters as function of the drop position inside the cavity.
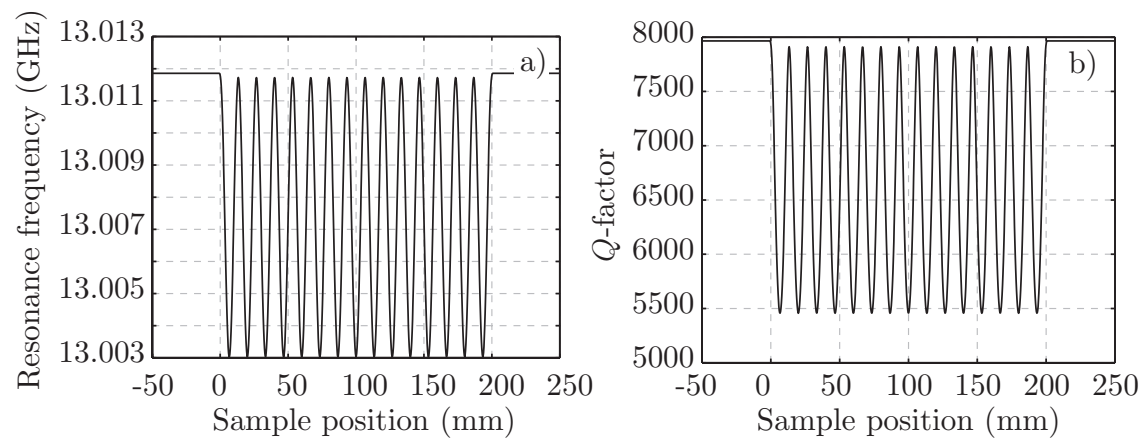

Figure 5.16. Calculated a) frequency and b) $Q$-factor deviation of a cavity while a HMTA cerium based solution $1.1 \mathrm{~mm}$ radius spherical sample falls through its central axis as function of drop position.

The maximal frequency deviation is in this case $8.82 \mathrm{MHz}$, while the $Q$-factor is reduced by $1 / 3$ from the empty cavity value, when the drop is at a maximum of the E-field.

The reflection parameter dependent from the position of the drop inside the cavity as well as the cavity input power, $P_{i n}$, are calculated with Algorithm 9 (see Page 112) from the frequency and quality factor values. The absorbed power $P_{d}$, which is represented in Figure 5.17 together with $P_{\text {in }}$, is calculated with Algorithm 10 (see Page 113).

To apply the absorbed power to the thermal model, a previous calculation of the HMTA solution thermal parameters is needed. HMTA has a 


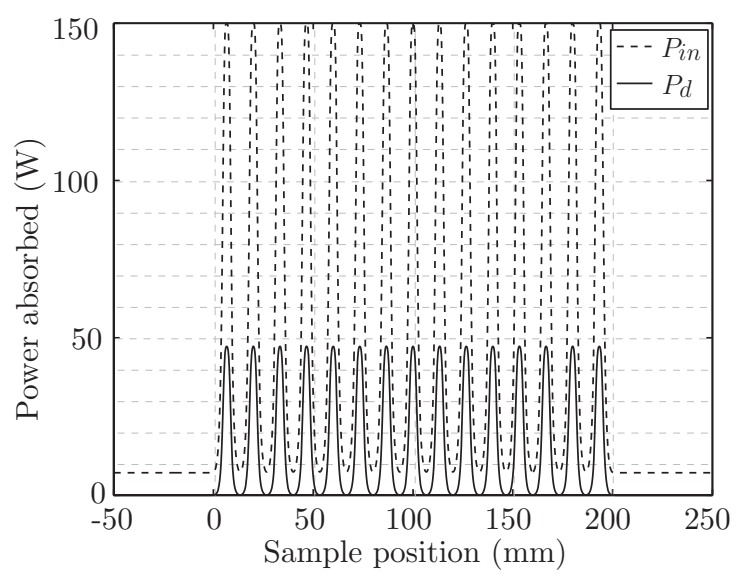

Figure 5.17. Calculated total absorbed power $P_{i n}$ and power absorbed by a $1.1 \mathrm{~mm}$ HMTA cerium based drop $P_{d}$ while falling through the cavity as function of sample position for $P_{\text {gen }}=150 \mathrm{~W}$.

solubility in water of $0.853 \mathrm{~kg} \cdot \mathrm{L}^{-1}$. That means that the mass fraction of HMTA in the solution is $\chi_{H}=0.4603$.

An approximation of the specific heat capacity for the solution can be calculated with the Kopp-Neumann law, which states that the molecular specific heat capacity of a compound is the sum of the atomic specific heat capacities of the elements composing it [85]:

$$
c_{p}=\sum_{i=1}^{N}\left(c_{p(i)} \chi_{i}\right)
$$

where $\chi$ is the mass fraction of the element. Knowing the density of HMTA $\left(1335 \mathrm{~kg} \cdot \mathrm{m}^{-3}\right)$ the specific heat capacity of this compound is calculated from data in [86] resulting in the value $1062.8 \mathrm{~J} \cdot \mathrm{kg}^{-1} \cdot \mathrm{K}^{-1}$. The water specific heat capacity is $c_{p}=4190 \mathrm{~J} \cdot \mathrm{kg}^{-1} \cdot \mathrm{K}^{-1}$. Consequently, the final solution specific heat capacity is $2750.6 \mathrm{~J} \cdot \mathrm{kg}^{-1} \cdot \mathrm{K}^{-1}$.

The densities of aqueous solutions are calculated from the mass fraction of hexamine in the mixture [87]:

$$
\rho=0.99707+0.2135 \chi+0.064 \chi^{2}
$$




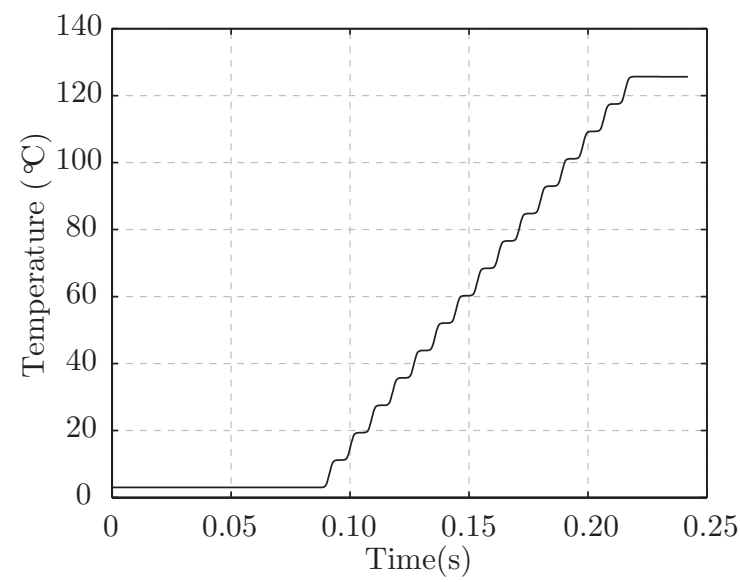

Figure 5.18. FDTD simulation of the heating rate for a 1.1 radius HMTA cerium based drop using a rectangular cavity (incident power $150 \mathrm{~W}$ ).

Accordingly, the density of the HMTA cerium based solution is $1108.9 \mathrm{~kg} \cdot \mathrm{m}^{-3}$

Because the main content of the HMTA cerium based solution is water, the water thermal conductivity is considered to solve the thermal model with the solution. The temperature of the drop as it is crossing the cavity has been analytically recovered by using Algorithm 11 (see Page 115) with $150 \mathrm{~W}$ of generator power.

To prevent a premature gelation, the solution is cooled to 3 degrees. Figure 5.18 represents the temperature profile as function of time since the drop generator $(t=0 \mathrm{~s})$ through the microwave rectangular cavity $(0.0869<t<0.2199 \mathrm{~s})$. It can be seen that the cerium solution reaches a higher temperature than the water solution for the same power (see Figure 4.16).

The maximal temperature of the cerium solution has been computed while sweeping the generator power from 0 to $150 \mathrm{~W}$ (Figure 5.19). Analytically it was found that about $100 \mathrm{~W}$ are enough to reach the $80 \pm 5^{\circ} \mathrm{C}$, which are necessary to trigger the internal gelation. 


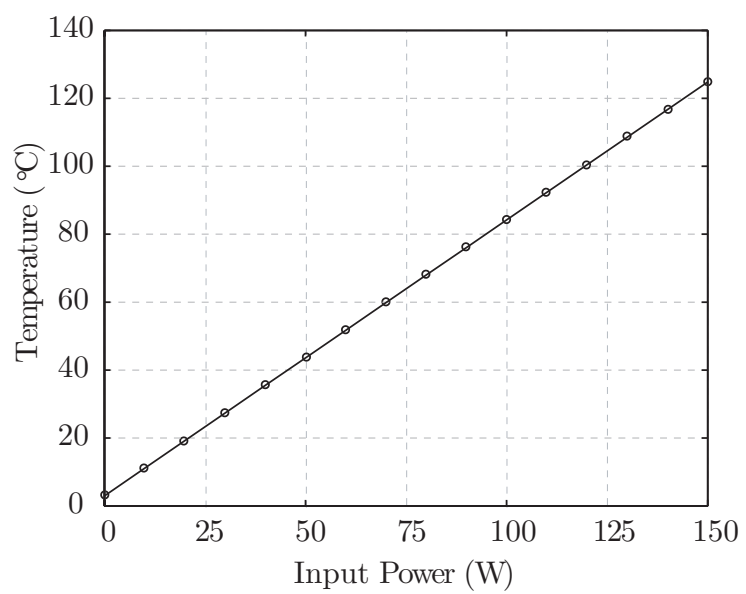

Figure 5.19. Measured temperature of a $1.1 \mathrm{~mm}$ HMTA cerium based drop (thermal properties: $c_{p}=$ $\left.2750.6 \mathrm{~J} \cdot \mathrm{kg}^{-1} \cdot \mathrm{K}^{-1}, \rho=1108.9 \mathrm{~kg} \cdot \mathrm{m}^{-3}, k_{T}=0.6 \mathrm{~W} \cdot \mathrm{m}^{-1} \cdot \mathrm{K}^{-1}\right)$ after its fall through the cavity as function of $P_{i n}$.

\subsection{Conclusions}

In this chapter the dielectric properties, which deal with the volumetric heating of materials have been introduced. Moreover, different techniques used for the measurement of dielectric properties have been presented. None of the techniques was optimal for the proposed PINE project. Consequently a new characterization method has been developed considering the geometry and the influencing parameters of the samples that should be produced in the context of the project.

The experimental setup has been described, including the adjustments like the sample access to the cavity, or the cavity coupling and working mode. The procedure to analyse the data after measurements has been explained and the shape behaviour of the recorded and processed signal has been discussed. The main materials involved in the project have been measured with the new method and the values have been validated with measurements (reported in previous literature) performed with other permittivity measurement techniques. 
The method has been satisfactorily proved. However further work is needed to decrease the runtime of the procedure, which is not attaining real time. In addition new research work could be done involving rectangular cavities instead of cylindrical. Working with higher modes in a rectangular cavity could be a possibility to get a better knowledge about the change on the dielectric properties of materials running chemical reactions, like in the case of gelation.

The measured dielectric properties of the PINE materials have been afterwards included in the perturbation and thermal models with the main intention of knowing the absorbed power by the material in the form of drops and the reached temperature. The results showed analytically that the system can produce gelated spheres.

The new characterization method was reported in [88]. A more detailed report has been submitted and peer reviewed by the Measurement Science and Technology journal. 



\section{MIG System}

6.1 Introduction . . . . . . . . . . . . . . . 153

6.2 MIG System description . . . . . . . . . . . . . . . 154

6.3 Equipment . . . . . . . . . . . . . . . . 156

6.4 Experimental Techniques . . . . . . . . . . . . . . 169

6.5 Results Analysis . . . . . . . . . . . . . . . . . 176

6.6 Conclusions . . . . . . . . . . . . . . . . . . . . 180 



\section{MIG System}

Previous CHAPTERs have presented physical principles involved in the microwave heating. In this chapter the applicators and their designs are introduced for the industrial practice of the high frequency heating applied to the PINE project. Furthermore the equipment which supplies the microwave energy to the cavities as well as its calibration and a power study considering its characteristics are presented. Finally, the experimental techniques and the results are reported.

\subsection{Introduction}

The devices which carry electromagnetic fields, like microwave cavities, employ metal conductors for the transmission from the generator to the load. The waves are guided inside hollow metal tubes till the resonator and then back to the termination load or detectors. Considering a perfectly conducting plane, the electric field component normal to the surface reach values while the component through the surface is zero. The magnetic field induces currents on the surface. 
However, these conductors can introduce losses to the transmitted signal when they do not perfectly match. Furthermore not all the devices present the same features, requiring in some cases the utilisation of other components to prevent the breakage of the whole system.

In order to anticipate failures each element needs an evaluation of its functionality in the system, on one side considering the manufacture specifications and on the other side experimentally proving those specifications because the devices usually come from different producers.

The following sections describe the whole procedure: from the decision of buying devices to the final experiments.

\subsection{MIG System description}

Although the microwave internal gelation requires heating to trigger the gelation of the solution in the system, this section also presents other setups which have been used during the development of this thesis.

The first setup (A) is the Non Heating Setup, which is represented in Chapter 5 (Figure 5.2). As it has been reported this setup is used on one hand to characterize the coupling of the cavities, and on the other hand to characterize materials in form of free falling drops while passing through the central axis of a cylindrical cavity.

The setup A is composed of three main parts:

- Drop Generator: to produce the water or feed solution drops that feed the cavity for the measurements.

- A microwave cavity connected to a Vector Network Analyser (VNA): the cavity has only one port for the input and output power coming from the VNA. The $S_{11}$ parameter of the cavity is analysed via this port, providing information about cavity resonances and the coupled signal.

- Drop Collector: to collect the drops after their passing through the cavity. 


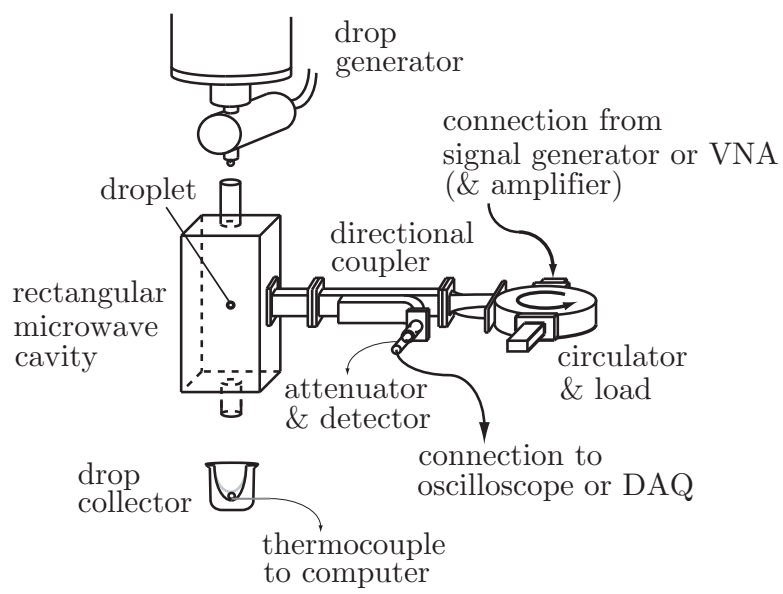

Figure 6.1. MIG Setup (B and C).

The second setup (B) is the Low Power Setup, which is used to characterize the power distribution of the cavities and to know how the electromagnetic waves interact with the drops inside. The setup, which is represented in Figure 6.1, consists of:

- A drop generator, a drop collector and a microwave cavity: like in setup A.

- A microwave generator: to produce the microwave signal and adjust the working frequency.

- A circulator and a termination load: The circulator directs the forward signal from the generator to the cavity and the backward signal from the cavity to the termination load and protects the amplifier from high reflected power.

- A directional coupler: couples a fraction of the back coming signal. From this signal it is possible to determine the reflected power, i.e. absorbed power.

- A crystal (detector): A Schottky diode is used to transform the coupled signal in voltage values. It is possible to add an attenuator be- 
tween the directional coupler and the detector to prevent overloading of the detector.

- A Data Acquisition Card (DAQ) or an Oscilloscope connected to a computer: to collect and save the data.

The third setup (C) is the Heating Setup, which is used for the microwave internal gelation, i. e. the production of gelled spheres by means of a microwave cavity which transmit the heating in form of electromagnetic waves. It consists of the same equipment than setup B but adding an amplifier to provide the cavity with the required power for heating.

\subsection{Equipment}

It is necessary to make a study of the technical characteristics of the cavity and all the elements that are connected to it in order to evaluate the electromagnetic signal and assure a system with minimal losses. In this section an exhaustive description of the components as well as the power study are presented.

\subsubsection{Design of the microwave cavity}

Three cavities are used during the experiments: a cylindrical cavity manufactured by ITACA (C1) which is fed through a loop (Figure 6.2 b)), a cylindrical cavity manufactured by PSI (C2) which is fed through an iris (Figure $6.2 \mathrm{c})$ ) and a rectangular cavity manufactured by ITACA (R1) which is also fed through an iris (Figure 6.4).

\section{Cylindrical cavity}

The utilisation of both X-band cylindrical cavities has been presented in Chapter 5. Figure 6.2 a) shows the simulation of the cylindrical cavity in the CST software [62] which has been used as well as the simulation in QW3D software [61] (see Figure 5.5) to calculate the electric field distribution and decide the cavity dimensions.

The operating resonant mode $\left(\mathrm{TM}_{010}\right)$, chosen due to its height invari- 

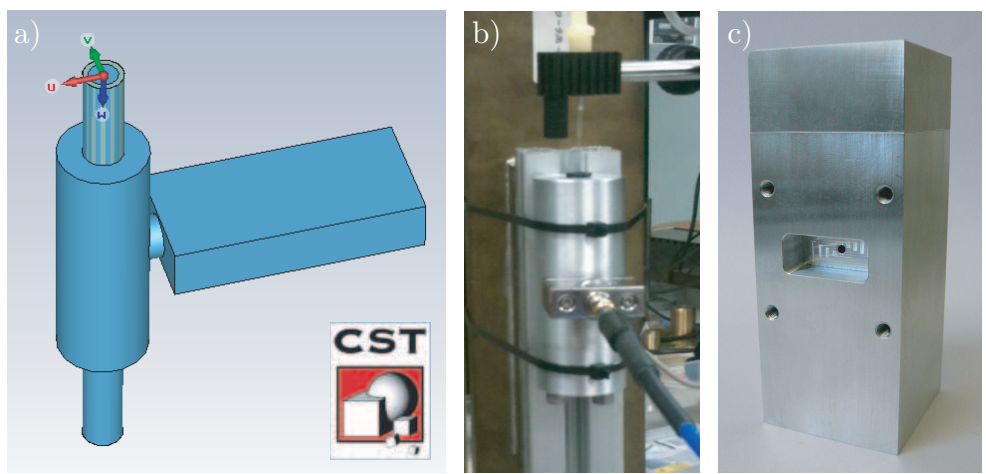

Figure 6.2. Cylindrical cavity: a) CST cavity simulation, b) ITACA cavity manufactured for low power measurements and c) PSI cavity manufactured with option of high power measurements.

ant electric field pattern, remains at the lowest frequency for a final cavity length of $50 \mathrm{~mm}$ (see Figure 4.9) avoiding the confusion with high order modes in the same frequency band.

Furthermore the CST simulation has been used to study the resonance frequency shift while a drop is moving along its central axis (Figure 5.10).

In order to be able to measure samples at different temperatures the second cylindrical cavity C2 (Figure $6.2 \mathrm{c}$ )) was designed. C2 has a refrigeration system in its walls, which was used during the dielectric properties measurement of HMTA cerium based solutions to prevent a premature gelation while experiments were done. The cavity is fed through an iris and a waveguide is transversally connected to it to generate the TM modes.

\section{Rectangular cavity}

Figure 6.3 shows the X-band rectangular cavity simulated with CST software. Thanks to the simulation the resonances of the modes and the E-field patterns generated for each mode have been determined. In Figure 6.3 a) the E-field pattern for the $\mathrm{TE}_{10(15)}$ mode is represented. Moreover, the simulation gives an overview of the field interaction with samples. Figure 6.3 b) presents the introduction of a water drop inside the cavity in a maximum 

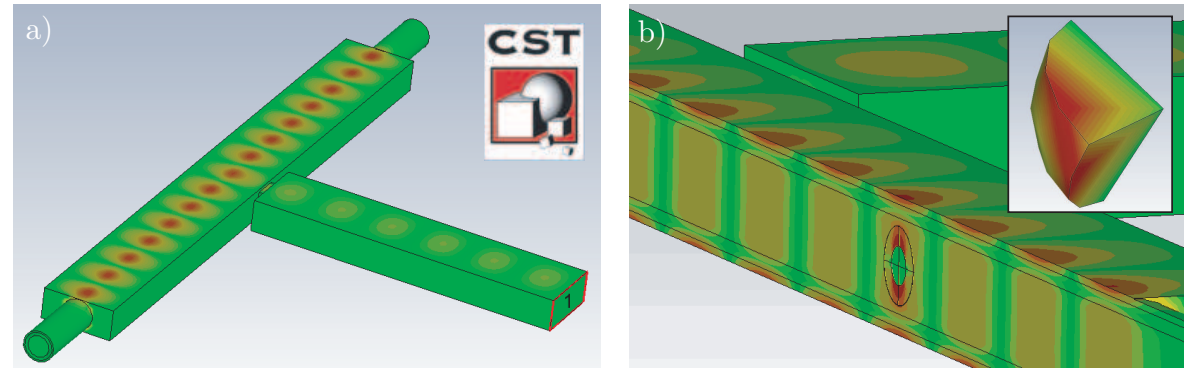

Figure 6.3. Simulation of the a) E-field distribution in a rectangular cavity and b) interaction of the field with a drop performed with CST software.

of the field pattern. By taking one eighth portion of the drop, it can be seen how the energy penetrates.

The final dimensions chosen for the rectangular designed cavity are $22.86 \times 10.16 \times 200 \mathrm{~mm}$ (Table 4.1 ). The cavity, which has been manufactured by ITACA, is shown in Figure 6.4 a).

The connected waveguide providing the signal through the iris ( $\mathrm{Fi}-$ gure $6.4 \mathrm{~b}$ )) should follow the verticality of the resonator to generate the
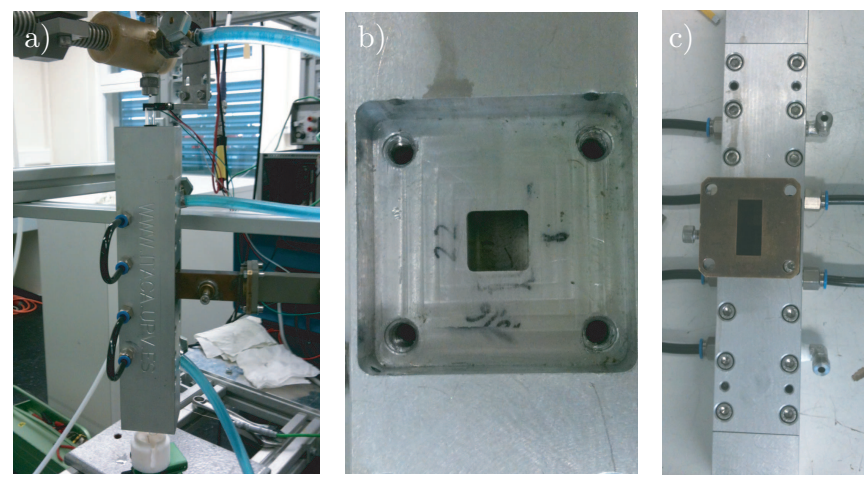

Figure 6.4. ITACA rectangular cavity manufactured for low and high power measurements: a) Cavity in the MIG system, b) iris, c) waveguide connexion. 


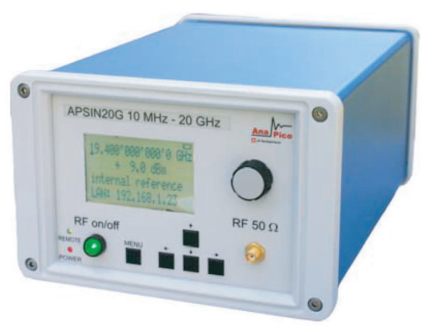

Figure 6.5. Microwave Generator.

transversal electric modes (TE) (see Figure $6.4 \mathrm{c}$ )). As the cavity is fed in the center, the generated modes will produce odd number of maxima in the electric field, e.g. $\mathrm{TE}_{10(15)}$.

Taking a deep insight on Figure 6.4 a) or c), one can identify a screw on the waveguide portion connected to the cavity. Due to the non changing iris dimensions, this screw will slightly tune the cavity during its calibration, to maximize the coupling of the signal.

The rectangular cavity is used for the microwave internal gelation system, to generate the electromagnetic waves, which will heat the samples. Then, as well as the cylindrical cavity $\mathrm{C} 2$, the rectangular cavity is provided with a cooling circuit within its walls, preventing high temperatures and dimensions changes.

\subsubsection{Microwave generator}

The microwave generator APSIN20G from Anapico (Figure 6.5) has been chosen for the MIG system. The generator offers a low-noise signal and fast-switching of the frequency covering a range from $100 \mathrm{kHz}$ up to 20 $\mathrm{GHz}$. It provides a $\mathrm{mHz}$ frequency resolution, with an output power range between -20 and $10 \mathrm{dBm}$ and includes AM, DC-coupled wideband-FM, PM, FSK and PSK, and fast pulse modulation as standard. Optionally, frequency chirps and programmable pulse trains can be executed.

The device can be also remote controlled, which is an advantage in case 


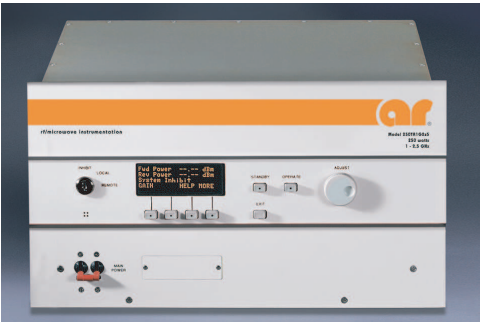

Figure 6.6. Amplifier.

it is placed in a glovebox.

\subsubsection{Amplifier}

As amplifier the Model 250TR7z5G18 from AR (Figure 6.6) was purchased. It offers 7.5 to $18 \mathrm{GHz}$ and up to $250 \mathrm{~W}$ power [89].

The Travelling Wave Tube Amplifier (TWTA) consists of two stages of RF amplification: a solid state preamp assembly with adjustable gain (EO 1175-000) and a traveling-wave tube amplifier (E08114-000). On the rear panel the input and output connectors are placed: The input connector is type $\mathrm{N} \mathrm{RF}$ and connects the generator to the preamp; the RF output connector from the TWT, which is directed through a straight length of waveguide and a 1800 E-plane bent into a dual -30 dB directional coupler, is a double ridge waveguide WRD-750 flange.

The reverse port on the directional coupler is connected to a detector diode, which provides information about the reverse power and protects the amplifier from excess values by measuring the VSWR (voltage standing wave ratio). The reverse power level should remain below $10 \%$ of the forward power or 20 watts $(+43 \mathrm{dBm})$, assuming that the load is properly matched.

The main specifications of the amplifier are:

- Power: 280 watts (nominal) and 250 watts (minimum)

- Flatness: $\pm 12 \mathrm{~dB}$ maximum 
- Frequency response: $7.5-18 \mathrm{GHz}$ instantaneously

- Input for rated output: $1 \mathrm{~mW}$ maximum

- Gain (at maximum setting): $54 \mathrm{~dB}$

- Gain adjustment: $35 \mathrm{~dB}$

- Impedance: 50 ohms input with VSWR 2.0:1 maximum and 50 ohms output with a typical VSWR 2.5:1.

The amplifier is controlled from the front panel. Two working procedures are possible: for the first procedure the gain can be fixed to a value in the amplifier and the power is changed step by step in the microwave generator (for a $100 \%$ gain, $0 \mathrm{dBm}$ should never be reached with the input drive to prevent unit damages); the second procedure consists on fixing the power value in the microwave generator to $0 \mathrm{dBm}$ while modifying the gain value on the amplifier.

The operation state of the amplifier can be also remotely controlled. However, as the microwave generator is the device from which the MIG system firstly depends (where the frequency is also specified), the first procedure has been selected for the PINE project experiments.

\subsubsection{Isolator}

The isolator is a model WRD750D24 from Space Machine 85 Engineering Corporation, which is composed of a double ridge ferrite circulator and a matched termination.

The circulator is a permanent magnet 3-port device that use a Yjunction structure housed in standard double ridge waveguide (WRD).

Figure 6.7 represents the isolator, which is connected to the amplifier through the flange $\mathrm{f}_{1}$ and to the cavity through the flange $\mathrm{f}_{2}$. The matched termination to the flange $f_{3}$ prevents an excess of forward power to be redirected to the amplifier.

The main specifications of the isolator are: 


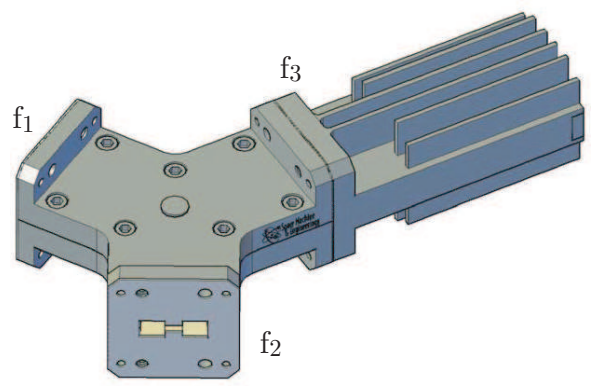

Figure 6.7. Isolator.

- Frequency response: $8-18 \mathrm{GHz}$

- Isolation: $15 \mathrm{~dB}$

- Insertion loss: $0.5 \mathrm{~dB}$ maximum

- VSWR: 1.40:1

- Maximum average power: $250 \mathrm{~W}$

- Maximum peak power: $2 \mathrm{~kW}$

\subsubsection{Waveguides}

Several waveguide elements or sections are used to connect the amplifier to the cavity. These elements facilitate the structure of the whole system and fit together the principal structures or devices when they have been designed for different frequency ranges or utilize different flanges. The elements used in the PINE project, most of which being from Space Machine \& Engineering Corporation, are shown in Figure 6.8.

\section{Transitions}

The MIG system amplifier as well as the circulator described above utilize WRD750 flange output because of their wide frequency working range. Nevertheless the microwave cavity is designed to work in a limited range of the X-band due to its length and thus has a rectangular waveguide WR90 
a)

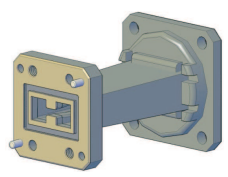

c)

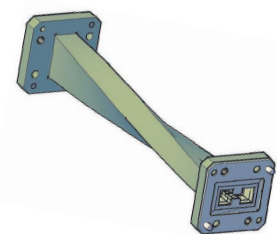

b)

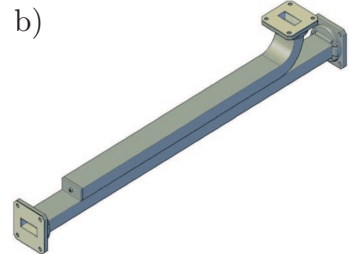

d)

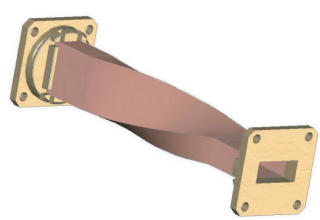

Figure 6.8. Waveguides: a) transition from WRD750D24 to WR90; b) directional coupler in WR90; c) 90 degree twist in WRD750D24; d) 90 degree twist in WR90.

flange input. Therefore, at some point of the structure, a transition from WRD750D24 to WR90 waveguide is required. This transition is represented in Figure 6.8 a).

\section{Directional Coupler}

A WR90 directional coupler (Figure $6.8 \mathrm{~b}$ )) is used in the system to recover and evaluate part of the forward power coming back from the cavity. Its features are:

- Frequency response: $8.20-12.40 \mathrm{GHz}$

- Directivity: $35 \mathrm{~dB}$ minimum

- Coupling: $20 \pm 0.5 \mathrm{~dB}$

\section{Twister}

Both the amplifier and the isolator use WRD750D24 flanges. However, the flange of the amplifier is vertical and the circulator has an horizontal flange. Because of the structural inconvenience of turning the isolator, a twist in WRD750D24 is necessary in the system (Figure 6.8 c)). Moreover, a second 
a)

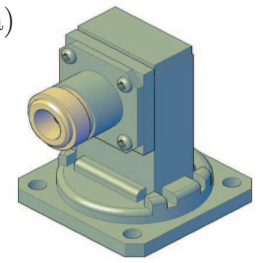

b)

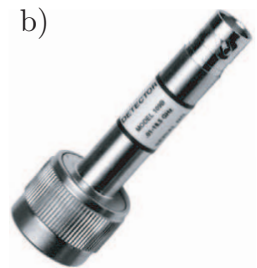

Figure 6.9. a) Adapter from WR90 to N. b) Detector

twist in WR90 is necessary after the circulator to follow the verticality of the cavity with the waveguide and couple the desired TE modes (Figure 6.8 d)).

\subsubsection{Adapter}

An adapter from WR90 to coax $\mathrm{N}$ termination (Figure 6.9 a)) is placed after the directional coupler, from which the power signal is required for analysis. The adapter works in the $8.20-12.40 \mathrm{GHz}$ frequency range.

\subsubsection{Crystal Detector}

A crystal detector, also known as Low-Barrier Schottky Diode (LBSD) detector converts the input power to an output voltage to treat the signal in an oscilloscope or with a data acquisition card in a computer. The detector used in the MIG system (Figure $6.9 \mathrm{~b}$ )) is the Zero Bias Schottky Detector model 109B from Krytar ${ }^{\circledR}$ with an N male input connector and a BNC female connector.

The detector specifications are as follows:

- Frequency range: $8.20-12.40 \mathrm{GHz}$

- Frequency response: $\pm 0.3 \mathrm{~dB}$ at $12.4 \mathrm{GHz}$ and $\pm 0.6 \mathrm{~dB}$ at $18.5 \mathrm{GHz}$

- Maximum VSWR: 1.15 at $4 \mathrm{GHz}, 1.3$ at $15 \mathrm{GHz}$ and 1.4 at $18.5 \mathrm{GHz}$

- Low level sensitivity: $0.5 \mathrm{~m} \cdot \mathrm{V} \cdot \mu \mathrm{W}^{-1}$

- Output capacitance: $30 \mathrm{pF}$ 
- Maximum input: $100 \mathrm{~mW}$

\section{Attenuator}

As well as the amplifier, both an oscilloscope and a VNA, used for the power signal evaluation, require a limited input voltage. Furthermore, the Crystal Detector has a maximum input level defined in its specifications and above this power level could be damaged. Then, an attenuator is needed between the adapter and the Crystal detector.

The attenuator has $\mathrm{N}$ input and output connectors (male and female respectively). Its specifications are:

- Frequency range: DC-18 GHz

- Maximum VSWR: 1.25:1 maximum

- Impedance: $50 \Omega$

- Attenuation: $20 \pm 0.5 \mathrm{~dB}$

- Input power: $2 \mathrm{~W}$ average @ $25^{\circ} \mathrm{C}$

- Peak Power: $250 \mathrm{~W}$ maximum

\subsubsection{Vector Network Analyser}

Two vector network analysers have been used during the experiments in the PINE project. The first one, the Agilent8720D (Figure 6.10 left), lent by the main partner EMPA Thun for the experiments in the PSI and the second one, the Rohde\&Schwarz ZVA 50 (Figure 6.10 right), provided by the ITACA group (main partner from the UPV València) during the experiments in the university.

Both amplifiers have been used for the coupling evaluation of the cavities and the characterization of materials with the cylindrical cavities, as seen in Chapter 5.

They cover the frequency range of the X-band and facilitate the amplitude and phase data recovery from the system. They can also be used 

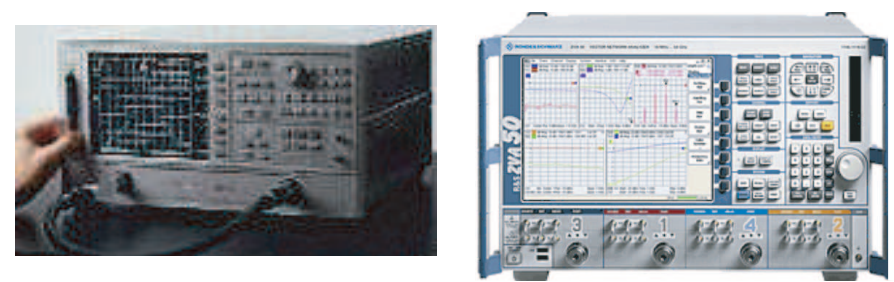

Figure 6.10. Vector Network Analysers.

as frequency generators, in such a way that the system S parameters can be recovered.

\subsubsection{Oscilloscope}

An alternative option to the VNA is the oscilloscope, with which by using a crystal only the voltage amplitude values coming from the forward signal can be recovered.

An oscilloscope GDS-2064 GW from Instek (Figure 6.11), which can be controlled remotely from a computer, has been utilised during the experiments.

The device displays the shape of the absorbed power signal produced when the samples pass through the cavity. Depending on this shape one can decide the adjustment of the frequency and power which have to be applied in the frequency generator.

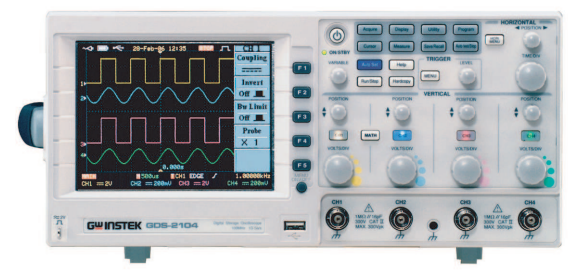

Figure 6.11. Oscilloscope. 


\subsubsection{Power requirements}

Transfer of energy from generator to workload must be achieved with a minimum loss. A high transfer efficiency is limited by two factors: the energy absorption in the walls and the power reflection from the load if this is not optimally matched. If the load resistance is less than the amplifier internal resistance, current flows back to the amplifier producing a dissipation that could damage the device.

The generator plus amplifier are connected through a circulator and a waveguide to the cavity. All the applicators should be adapted, and a power study should be performed in order to avoid a loss of efficiency from the generator to the workload, i.e. the cavity heating the sample, and to avoid damage of the generator and/or amplifier in case there is no workload dissipating the energy.

Usually the load resistance and/or the generator internal resistance are not the same values as the waveguide characteristic impedance $Z_{0}$ and reflected waves $V^{-}$appear in the transmission line together with the transmitted waves $V^{+}$. The relation between the waves is known as reflection coefficient

$$
\Gamma=\frac{V^{-}}{V^{+}}
$$

This coefficient defines the transmitted power as seen in equation 4.90 The interference between both waves generates cases of maximum and minimum voltage. The ratio between both cases is known as Voltage Standing Wave Ratio (VSWR), which could be measured by taking the reflected wave with a directional coupler.

$$
S=\frac{1+|\Gamma|}{1-|\Gamma|}
$$

A power balance has been performed to determine the input power (generator) needed for the experiments and to prevent the rest of the equipment from damage. To carry out this balance the specifications of each element in the setup (coupling, gain, attenuation) have been used. 
The first power balance is performed to ensure a power between $100 \mathrm{~W}$ $(50 \mathrm{dBm})$ and $250 \mathrm{~W}(54 \mathrm{dBm})$ inside the microwave cavity. Since the amplifier (working with $100 \%$ gain) has a minimum output power of $54 \mathrm{dBm}$ the theoretically required values at the source are the ones presented in Table 6.1.

\begin{tabular}{lcc}
\hline & $\mathbf{1 0 0} \mathbf{~ W}$ & $\mathbf{2 5 0} \mathbf{~ W}$ \\
\hline Power in the cavity & $50 \mathrm{dBm}$ & $54 \mathrm{dBm}$ \\
Amplifier & $54 \mathrm{dBm}$ & $54 \mathrm{dBm}$ \\
Source & $-4 \pm 6 \mathrm{dBm}$ & $0 \pm 6 \mathrm{dBm}$ \\
\hline
\end{tabular}

Table 6.1. Power balance from amplifier to cavity.

The second power balance is performed to prevent the amplifier from damage. The maximum reverse power should be $+43 \mathrm{dBm}$. If no power is absorbed in the cavity, a maximum forward power of $54 \mathrm{dBm}$ comes back from the resonator. The circulator has an isolation of $-15 \mathrm{dBm}$. Then, the maximal reverse power at the amplifier is $39 \mathrm{dBm}$, which is less than the value indicated in the specifications, and therefore is safer.

The third and final power balance is performed to prevent the detector from damage. The allowable power levels for the detector are -30 to $+18 \mathrm{dBm}$. The coupling from the directional coupler is $20 \mathrm{dBm}$ and the attenuator lower the signal also by $20 \mathrm{dBm}$. Then, for a maximum forward power of $54 \mathrm{dBm}$, the maximal input power in the detector is $14 \mathrm{dBm}$, value which is in the functional range.

\subsubsection{Calibration}

When working with a VNA, the analyser should be calibrated in the frequency range of the designed microwave cavity before the measurements; new calibrations should be made each time the frequency range utilized in the analyser is changed.

The calibration kit used to calibrate the analyser is the Rosenberger RPC-N 50 that works in the frequency range from $0 \mathrm{GHz}$ (DC) to 18 GHz. The analyser should be calibrated with the charges provided in the 
calibration kit (open, short and load).

If the Smith chart (SC) is used to calibrate the analyser, working in impedance mode, the open circuit should appear on the right SC semicircumference, and it should be a point when the reflection factor $(\Gamma)$ equals 1 . The short circuit should appear on the left SC semicircumference and it should be a point when $\Gamma$ equals -1 . The adapted load appears as a point in the center of the Smith chart, where $\Gamma$ equals 0 . Working in admittance mode the chart would be the same but $180^{\circ}$ rotated.

\subsection{Experimental Techniques}

The experimental techniques used for the characterization of materials have been presented in Chapter 5. In this section the experimental techniques used for the microwave internal gelation are presented.

\subsubsection{Rectangular cavity characterization}

Once the cavity is manufactured it needs a characterization to identify its modes with the resonance frequencies. The aperture dimensions are decided with the foresight of the working frequency selection. In this case the iris is a centered $8 \mathrm{~mm}$ side square, and the coupling will be slightly tuned thanks to a screw coupled to the connected waveguide section.

By using as a reference the frequency response of the simulated rectangular cavity with CST software, shown in Figure 6.12, the most interesting modes for the heating of samples are researched with the aid of a VNA. The representation corresponds to a simulation, which has not converged and which has been used to identify the positions of the modes.

As described before, first the Agilent8720D VNA is calibrated in the $\mathrm{X}$-band frequency range, which as in the case of the simulation will be 8 to $14 \mathrm{GHz}$. Afterwards the modes and frequencies are recognized for the case of the empty cavity and the cavity with quartz, by following the shift of the frequency while inserting it. The frequencies are reported in Table 6.2. 


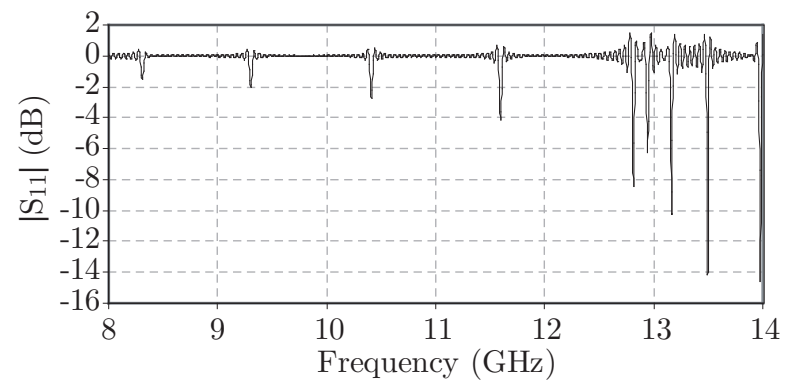

Figure 6.12. CST rectangular empty cavity frequency response.

\begin{tabular}{lcc}
\hline & $\mathbf{T E}_{10(13)}$ & $\mathbf{T E}_{10(15)}$ \\
\hline Empty Cavity & $11.7476 \mathrm{GHz}$ & $12.9434 \mathrm{GHz}$ \\
Cavity with quartz & $10.0001 \mathrm{GHz}$ & $11.0514 \mathrm{GHz}$ \\
\hline
\end{tabular}

Table 6.2. Resonance frequencies of the rectangular cavity.

Several measurements of the input return losses were carried out while tuning the screw on the waveguide to evaluate the coupling of the cavity.

For the case of the cavity with quartz and mode $\mathrm{TE}_{10(15)}$ at $11.0514 \mathrm{GHz}$, the module and phase representation of the input return losses while the screw is introduced is shown in Figures 6.13 and 6.14 respectively. The measurement was also carried out for the cavity without quartz, and for the other modes.

The position of the screw is decided to have an overcoupling of the input return losses as foresight of the undercoupling coming from the samples passing through the cavity. Therefore the sample will reduce the coupling to work close to an ideal critical coupling situation (circumference crossing the center of the Smith chart) and to ensure an efficient transmission of the energy. 


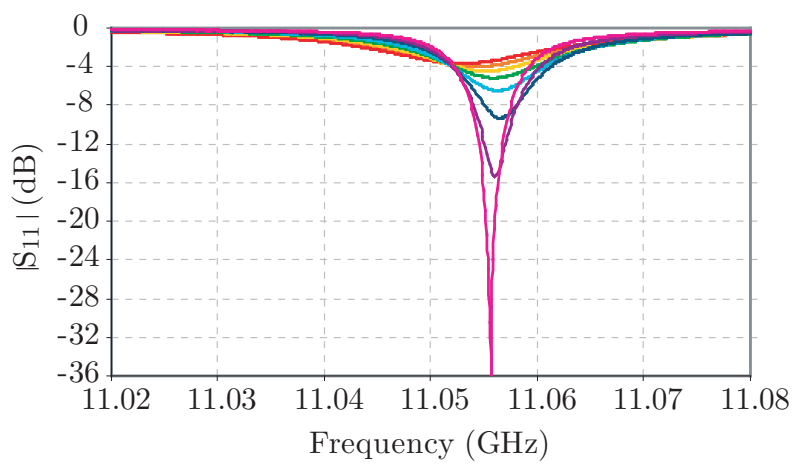

Figure 6.13. Coupling measurements on rectangular cavity: $\left|S_{11}\right|$ module depending on the coupling dictated by the insertion of the screw. The deeper the screw, the stronger the resonance and the higher its frequency shift.

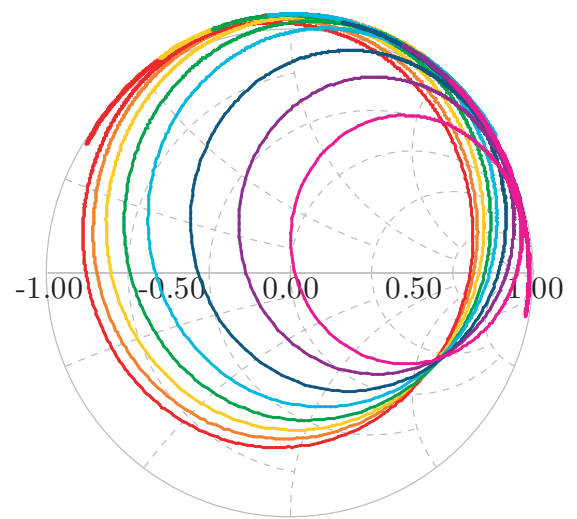

Figure 6.14. Coupling measurements on rectangular cavity: $\left|S_{11}\right|$ phase depending on the coupling dictated by the insertion of the screw. The deeper the screw, the smaller the circumference on the Smith chart.

\subsubsection{MIG System Assembly}

Like for the cavity, some of the devices and components described in the previous section are characterized before the system assembly. 


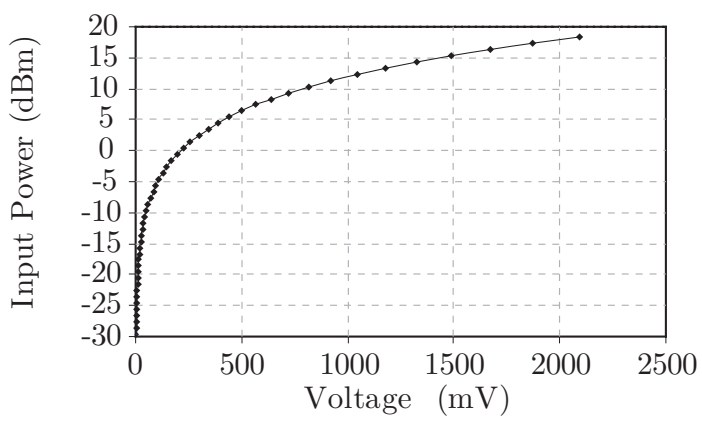

Figure 6.15. Detector characterization curve.

The first component to characterize is the one which will provide the reading of the reverse signal to recover the absorbed power, i.e. the detector. Usually its characterization is indicated in the property specifications of the provider. However the element could have a deviation produced during the manufacture or later, and should be characterized anew before usage. The detector is connected to a microwave generator at $10 \mathrm{GHz}$ and it is exposed to power within the specified range. For each power value, the voltage values are recovered from a voltmeter. The measured output voltage vs. input power curve is represented in Figure 6.15.

As it is important to know the real power entering the cavity, an experiment is done connecting the directional coupler in a way to recover the power that is going inside the cavity and compare it with the values that the amplifier displays.

Figure 6.16 gathers these measurements. It can be seen a non constant gradient in the representation, only in the region (a) around 50-100 W, the amplifier displays values close to the measured ones. Principally, to protect the system from damage and failure, it has to be considered that from $100 \mathrm{~W}$ on, the power entering the cavity is higher that the one displayed on the amplifier. 


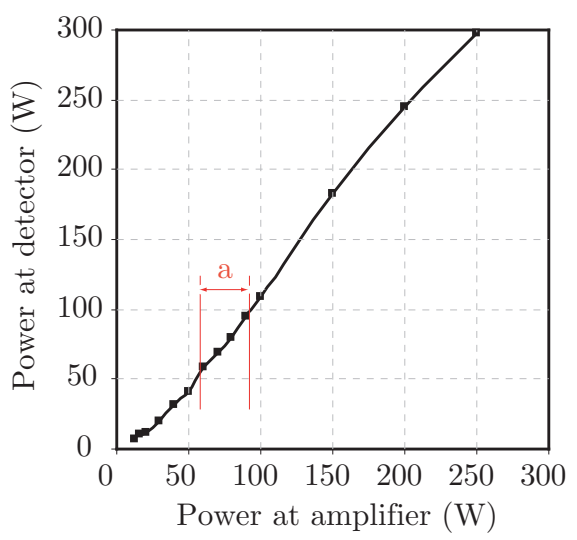

Figure 6.16. Input power at amplifier vs. input power going to the cavity (measured with the detector).

Once the devices are characterized, the system is assembled and prepared for the experiments. Figure 6.17 shows the MIG system, which is placed in the Hotlab of the Paul Scherrer Institut in Villigen, Switzerland. The reason to place the system in such a laboratory is the possibility of using it (after approval) with radioactive solutions, derived from spent nuclear fuel, and produce the SP-fuel. Furthermore, the laboratory is a zone where the external conditions can be controlled.

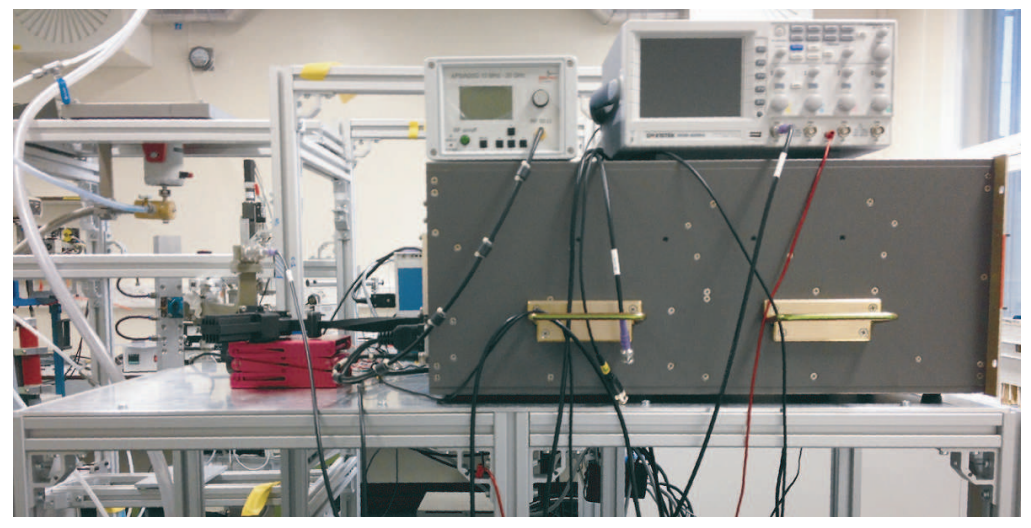

Figure 6.17. MIG system assembly in the Hotlab of the Paul Scherrer Institut. 
In addition to the microwave related devices and components, the MIG system consists of the drop generator from which the prepared solution is precipitated to the cavity. The solution conditioning for the MIG system is next described.

\section{Broth Preparation}

As explained in Section 2.4.3 describing the chemistry of the internal gelation, the gelation occurs when the broth reaches a given $\mathrm{pH}$. The sensitivity with which the broth will gel thus depends on the initial $\mathrm{pH}$ of the broth and the potential for a temperature induced $\mathrm{pH}$ shift (i.e. the amount of HMTA). The initial $\mathrm{pH}$ of the broth is determined by the amount of base ( $\mathrm{NaOH}$ in the current work) mixed with the metal nitrate solution. The magnitude of the temperature induced $\mathrm{pH}$ shift is obtained by controlling the concentration ratio between the HMTA and the metal nitrate. Because of its premature gelation preventing role (that is before heating), the urea concentration is critical when mixing the solutions.

Figure 6.18 schematizes the solution preparation and the drops generation and heating. The broth is filled into a cooled tank with a stirring device. Thanks to pressurized air, the broth is pushed toward a nozzle with a given diameter, which is chosen according to the targeted final drop diameter. The initially sessile drop forming at the nozzle falls by gravitation. In order to avoid premature gelation, the temperature of the broth from the tank to the nozzle has to be controlled. Therefore, a double wall tank and a feeding pipe are used, which are connected to a cooling unit. The temperature of the coolant is set between $0^{\circ} \mathrm{C}$ and $5^{\circ} \mathrm{C}$. Although this work focuses on the generation of large droplets, smaller spheres can be produced in order to obtain a high fuel density in the Sphere Pac concept [90] with only slight changes, such as a higher air pressure and the use of a vibrator. 


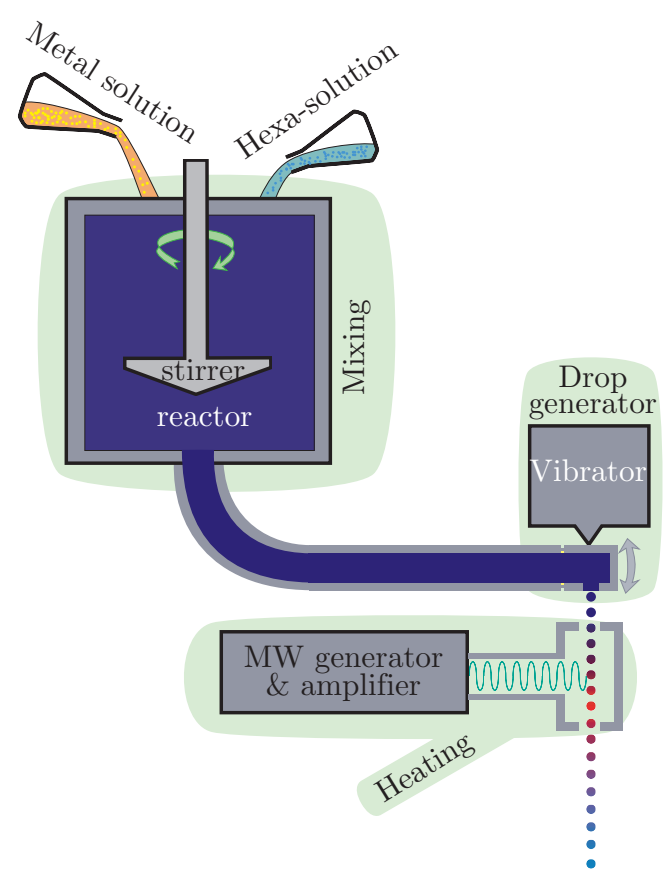

Figure 6.18. Schema broth preparation, generation of drops and microwave heating.

\subsubsection{Heating Experiments}

Two kinds of heating experiments are implemented in the PINE project. First the system is tested by heating water. Once the water temperature is increased to the gelation minimum temperature, the heating experiments with the HMTA solution are carried out.

The water temperature is measured with two different methods, depending on the laboratory facilities. On one side a thermograph camera, model ThermaCAM FLIR E45, was shared by the ITACA group, on the other side a thermocouple connected through a DAQ to the computer was assembled in the PSI Hotlab.

The protocol followed during the heating experiments is represented in a schema in Figure 6.19. This protocol consists of water drop measurements while increasing the power stepwise. At the same time the power 


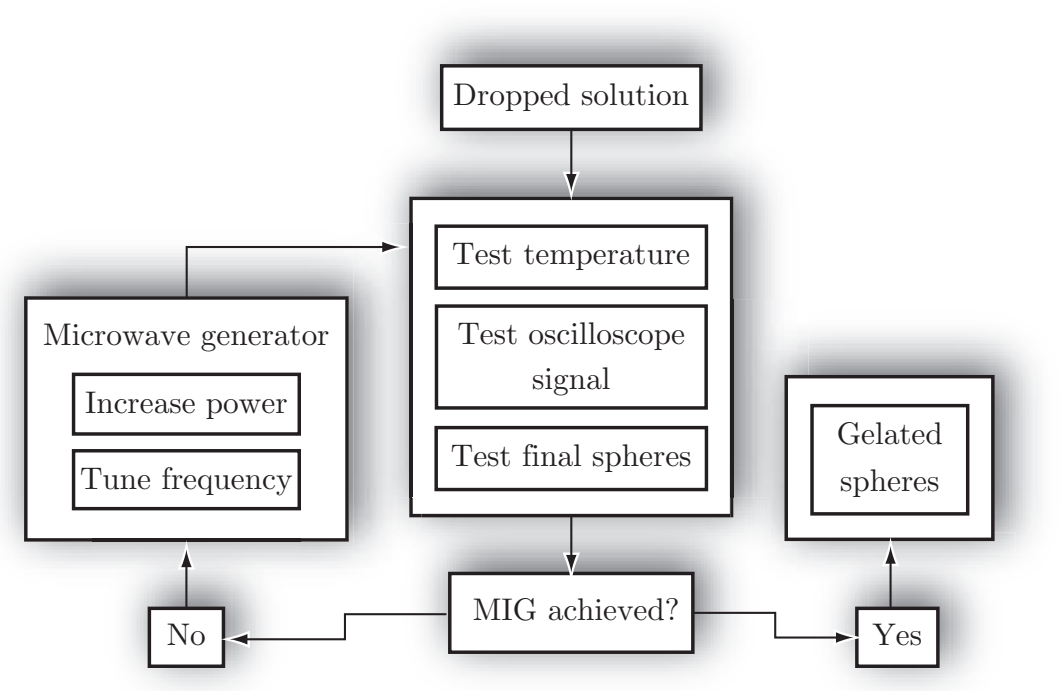

Figure 6.19. Schema of the heating experiment protocol.

coming back from the cavity is redirected through the directional coupler to the detector, where it is converted to voltage values and displayed on the oscilloscope. The shape of the represented signal helps with the readjustment of the frequency and power in the generator. Furthermore the signal informs about possible failures in the system, which make the heating of the samples impossible, e.g. wet or dirty quartz.

The gelation experiments will start with the measured power which increases the water temperature up to 60 degrees. Afterwards the power is increased and/or the frequency slightly changed by following the same procedure as for the water, based on the oscilloscope recovered signal.

\subsection{Results Analysis}

Several measurements were carried out with water. Figure 6.20 show temperature measurements, which were taken, during the experiments in the UPV, with the thermograph camera for the case of the cavity working with 


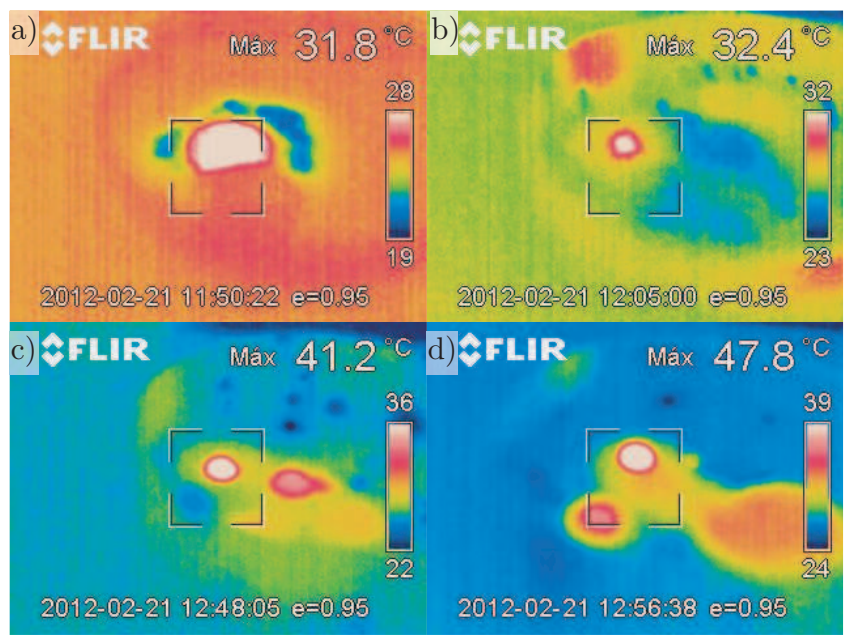

Figure 6.20. Thermal captures of water drops with ThermaCAM FLIR E45.

$\mathrm{TE}_{10(13)}$ mode at $9.9883 \mathrm{GHz}$. The captures a) and b) correspond to a $42 \mathrm{dBm}(15.84 \mathrm{~W})$ input power, while captures c) and d) correspond to a $47.5 \mathrm{dBm}(56.23 \mathrm{~W})$ input power. The central oval with the highest temperature level represents the drop after its passage through the cavity, collected on an insulated thin plate. $2.9 \mathrm{~mm}$ diameter droplets were heated up to 50 degrees with less than 60 watts.

During the experiments in PSI, the temperature measurements were carried out for two different modes $\mathrm{TE}_{10(13)}$ at $9.9883 \mathrm{GHz}$ and $\mathrm{TE}_{10(15)}$ at $11.0470 \mathrm{GHz}$. Figure 6.21 represent the temperature measured with the aid of a thermocouple placed at the bottom of a cup made of insulating material. The uncertainty represented in the case of the experimentally captured values is a result of the measurement method: the drop is collected $20 \mathrm{~cm}$ below the cavity in a cup made of insulating material, at the bottom of which a thermocouple is placed. The drops were generated at room temperature $\left(25{ }^{\circ} \mathrm{C}\right)$ for this experiment.

These temperature measurements are also used in order to verify the analytical approach presented in Section 4.6.4. Figure 6.22 represents the measured values for the $\mathrm{TE}_{10(15)}$ mode (dashed line) together with the 


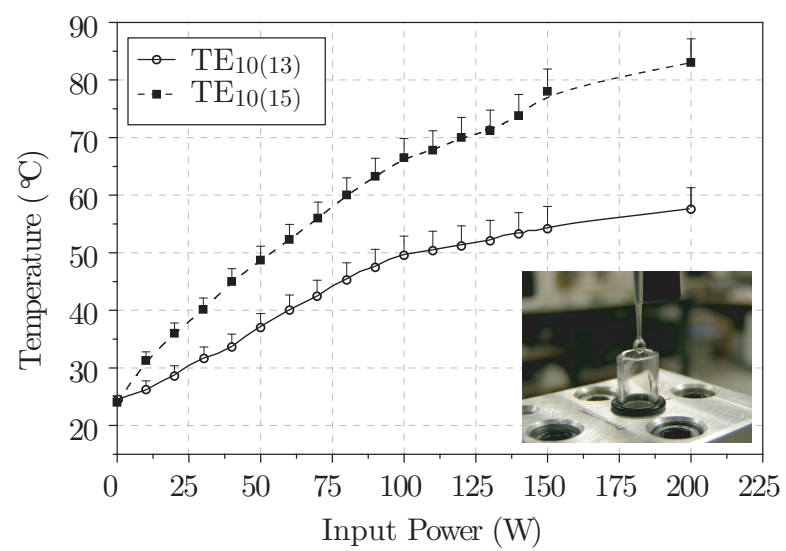

Figure 6.21. Temperature of a $1.1 \mathrm{~mm}$ water drop (thermal properties: $c_{p}=4190 \mathrm{~J} \cdot \mathrm{kg}^{-1} \cdot \mathrm{K}^{-1}, \rho=1000 \mathrm{~kg} \cdot \mathrm{m}^{-3}, k_{T}=$ $0.6 \mathrm{~W} \cdot \mathrm{m}^{-1} \cdot \mathrm{K}^{-1}$ ) after its fall through the cavity as function of $P_{i n}$, for two different modes.

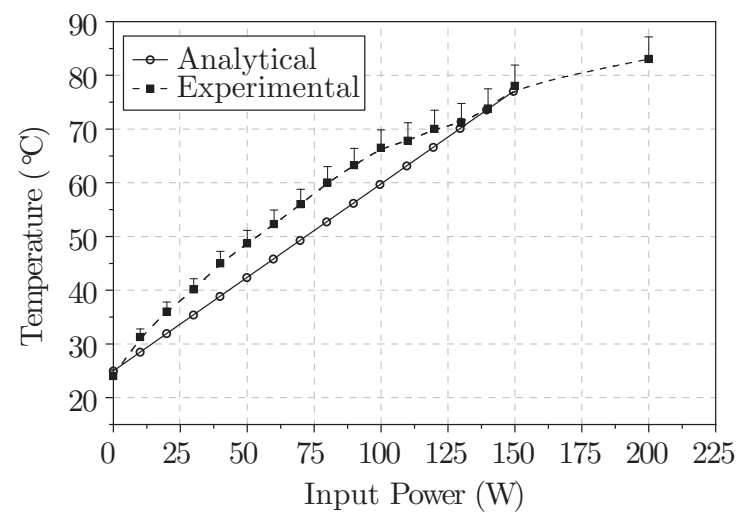

Figure 6.22. Temperature of a $1.1 \mathrm{~mm}$ water drop after its fall through the cavity as function of $P_{i n}$.

analytical calculated temperature values (plain line) considering in both cases the same input powers (marked points).

The temperature for $P_{i n}=150 \mathrm{~W}$ in the experimental measurements is around $78^{\circ} \mathrm{C}$ which is close to the analytical maximum value $\left(78.31{ }^{\circ} \mathrm{C}\right)$ 
calculated with the model and represented in Figures 4.18 and 6.22.

The results confirmed that the water reaches the gelation temperature with the $\mathrm{TE}_{10(15)}$ mode at $11.0470 \mathrm{GHz}$ and a power around $150 \mathrm{~W}$. As a consequence, this mode is selected for the gelation experiments with the HMTA cerium based solution.

During the gelation experiments execution there is an initial stabilization period of the system. According to the signal indicated on the oscilloscope during the first 5 minutes the frequency and the power should be changed continuously in the frequency generator. After this time the cavity approaches a temperature equilibrium and the working frequency starts to stabilize at a lower value compared to the startup condition. After 10 minutes the system does not need further calibration and gelated spheres are produced continuously.

As an example, one of the experiments was starting with the frequency tuned to $11.0767 \mathrm{GHz}$ and a power of $52.2 \mathrm{dBm}$. The stabilized production was finally carried out with a resonant frequency of $11.063 \mathrm{GHz}$ and a power of $51.8 \mathrm{dBm}$.

When microspheres from the cerium solution are fabricated, they are collected in a metal basket placed in a beaker. The beaker is filled with water in order to reduce the impact of the soft spheres. Figure $6.23 \mathrm{a}$ ) shows the collector beaker, while Figure $6.23 \mathrm{~b}$ ) shows a picture of the as collected spheres, before they are dried, aged in ammonia or thermally treated. The average size of a sphere is $2 \mathrm{~mm}$ diameter.
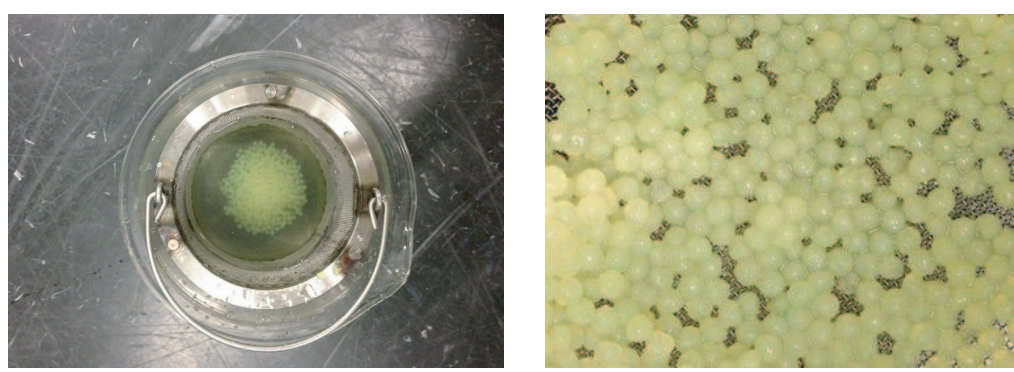

Figure 6.23. Cerium gelled spheres collected in water. 


\subsection{Conclusions}

In this chapter, the MIG system for the production of gelated particle spheres was described. The devices which take part in the setup were presented together with their main specifications that were useful to fit together the system. The implementation of the microwave cavities, both simulations and manufactures by PSI and UPV were explained. The CST software facilitated the recognition of modes while representing the fields. It also offered an insight of the interaction of drops with the field within the cavities.

In the case of the rectangular cavity, a characterization was carried out with a VNA for the experimental research of the modes, starting from the resonant frequencies given by the CST software. Moreover, the coupling by means of a tuning screw for a fixed iris was evaluated.

The assembly of the MIG system in the hotlab of the PSI was presented and the different techniques for the heating experiments were explained. The results of the temperature measurements as well as the gelation experiments were reported. The HMTA cerium based gelled spheres proved the satisfactory solution of considering microwave cavities for the internal gelation procedure.

The main contributions of this chapter were published in [91]. 


\section{Conclusions}

7.1 Summary . . . . . . . . . . . . . . . . . . . . . . 184

7.2 Further Work . . . . . . . . . . . . . . . . . . . . . 185

7.3 List of Publications . . . . . . . . . . . . . . 186 



\section{Conclusions}

ThE OVERALL AIM of this research was to develop an advanced system capable to produce Sphere-Pac fuel by microwave internal gelation. The motivation of this research came from the necessity of reducing the large amount of liquid waste while simplifying the production unit in comparison with the production of the Sphere-Pac fuel using the previously silicon oil based technique.

This chapter will summarize the findings of this research work, revisiting the research objectives given in the introductory chapter. First, Section 7.1 will review the contents of this study, outlining the main conclusions that were extracted from each chapter. Recommendations for future research will be discussed in Section 7.2. Additionally, the final section contains a list of work published as well as a list of conferences, seminars and courses carried out during the course of candidature for the degree. 


\subsection{Summary}

The first part of this dissertation presented a complexity study of the microwave internal gelation for the production of particle fuel. An overview of nuclear fuel manufacture was researched getting deeper in the production methods for particle fuel. The chemistry was treated and the previous research related with the internal gelation was described.

Chapter 3 presented the performance of the thermal model when it is applied for the study of the material heated by microwaves. The model was implemented with the FDTD technique applying boundary conditions to adjust the model to real conditions. The thermal variables of the formulation were evaluated for a deeper knowledge of the model behaviour. Two validations were applied to the thermal model for its evaluation confirming the consistence of the model.

Chapter 4 introduced both rectangular and cylindrical cavities with their field equations and the calculation of their parameters, i.e. resonant frequency and quality factor, as well as the calculation of their energies and powers for the case of empty cavities and cavities loaded with dielectrics. The coupling system used to feed the cavities was shown to be crucial to transfer the power efficiently. Several software packages for the design of the cavities were commented. Specifically the numerical Matlab software was utilized to compute cavity models, including the perturbation method, which updated the thermal model presented in Chapter 3, with the cavity parameters.

Chapter 5 presented the dielectric properties, which deal with the volumetric heating of materials. Different techniques used for the measurement of dielectric properties were introduced and a new characterization method was developed considering the geometry and the influencing parameters of the samples that should be produced in the context of the project. Both the experimental setup and the data analysis were explained, and the method was utilized to measure the permittivity of the main materials involved in the project, which was included in the perturbation and thermal models with the main intention of knowing the absorbed power by the material in form of drops and the reached temperature. 
Chapter 6 described the MIG system for the production of gelated particle spheres, starting with the specifications of its devices and finishing with its assembly. The implementation of the microwave cavities, both simulations and manufactures by PSI and UPV, were explained. In the case of the rectangular cavity, a characterization was carried out with a VNA for the experimental research of the modes with their resonant frequencies and the cavity coupling. The different techniques for the heating experiments were explained. The results of the temperature measurements as well as the gelation experiments were reported. The obtaining of HMTA cerium based gelled spheres demonstrated the feasibility of MIG and justified the consideration of microwave cavities for the internal gelation procedure.

\subsection{Further Work}

Following the investigations described in this thesis, the main lines of research that remain open are listed below:

- Characterization of materials in X-band cavities: A new research work could be done involving either rectangular or cylindrical cavities for the characterization of the permittivity. Working with more than one variation in the $z$ direction of the cavity could possibly provide a better knowledge about the change on the dielectric properties of materials undergoing chemical reactions with temperature, such as the gelation.

- Remote operation: To enhance the safety of the operator, the operations should be remotely performed. To do so, all the mechanic, electronic and electric components are chosen in a way that they can be centrally controlled from a computer. A computer interface tool (i.e. Labview) is foreseen to turn on/off the unit and tune each parameter separately. In a more distant future, an optimization routine can be used to follow up the reflected signal from the cavity and find the best heating parameters.

- Tranfer to a glovebox: the current tests are carried out with an in- 
active surrogate, the equipment is chosen and installed in a way as close as possible to the final production unit where actinides will be handled. To prevent contamination of the environment, all the instruments directly in contact with the broth have to be placed in a sealed environment; therefore, the transfer of the production unit into a glovebox is being studied [91].

\subsection{List of Publications}

The following list presents the published works produced during the course of candidature for the degree. None of the publications have previously formed a part of another thesis.

\section{Refereed ISI Journals}

- M. Cabanes-Sempere, J.M. Catalá-Civera, C. Cozzo, S. Vaucher, M.A. Pouchon, "Innovative production of nuclear fuel by Microwave Internal Gelation: Heat transfer model of falling droplets", Prog. $\mathrm{Nu}$ clear Energy, vol. 57, pp.111-116, May 2012

- M. Cabanes-Sempere, J.M. Catalá-Civera, F.L. Peñaranda-Foix, C. Cozzo, S. Vaucher, M.A. Pouchon, "Characterization method of dielectric properties of free falling drops in a microwave processing cavity and its application in Microwave Internal Gelation", Measurement Science and Technology, submitted January 2013, peer-reviewed and resubmitted May 2013.

\section{Papers in International Conferences}

- M. Cabanes-Sempere, J.M. Catalá-Civera, C. Cozzo, S. Vaucher, M.A. Pouchon, "Innovative production of nuclear fuel by Microwave Internal Gelation: Theoretical microwaves-microspheres interaction", Proceedings of the 13th International Conference on Microwave and RF Heating, AMPERE 2011, September 5-9th, 2011, Toulouse, France, pp. 231-234. ISBN: 978.2.85428.978.7 
- M. Cabanes-Sempere, C. Cozzo, J.M. Catalá-Civera, F.L. PeñarandaFoix, K. Ishizaki, S.Vaucher, M.A. Pouchon, "Characterization of Free Falling Drops inside a Microwave Cavity", Proceedings of the International Microwave Symposium IMS 2012, June 17-22nd, 2012, Montreal (Canada), pp. 1738-1740. ISBN: 978.1.4673.10868.4. (DOI: 10.1109/MWSYM.2012.6259757)

- C. Cozzo, M. Cabanes-Sempere, M.A. Pouchon, "Method of Advance Waste Conditioning by Microwave Internal Gelation: Set Up Development and Modeling", Actinide and Fission Product Partitioning and Transmutation, 12th Information Exchange Meeting, Prague, Czech Republic, 24-27th September, 2012.

- M. Cabanes-Sempere, A.J. Canós, J.M. Catalá-Civera, F.L. PeñarandaFoix, C. Cozzo, M.A. Pouchon, "Power and Thermal Study of a Single Drop Heated while passing through a $\mathrm{TE}_{1} 0 p$ Rectangular Cavity", Proceedings of the International Microwave Symposium IMS 2013, June 2-7th, 2013, Seattle (USA), pp. 1-4. ISBN: 978.1.4673.2141.9

\section{Others}

- M. Cabanes-Sempere, C. Cozzo, S. Vaucher, J.M. Catalá-Civera, M.A. Pouchon, "Innovative production of nuclear fuel by Microwave Internal Gelation: Heat transfer model of falling droplets", Keynote at Symposium V: Nuclear materials of the E-MRS 2011 Spring $\mathcal{E}$ Bilateral Meeting, Nice, France, 9-13th May, 2011.

- M. Cabanes-Sempere, "Nuclear fuel production by microwave internal gelation: Heat transfer model", Poster presented at NES PhD Day 2011, Paul Scherrer Institut, Villigen, Switzerland, 8th June, 2011.

- C. Cozzo, M. Cabanes-Sempere, M.A. Pouchon, "Reduktion der Radiotoxizität des Kernabfalls", Poster presented at PSI open doors, Paul Scherrer Institut, Villigen, Switzerland, 16th October, 2011.

- C. Cozzo, M. Cabanes-Sempere, M.A. Pouchon, "Herstellung von Brennstoff durch internale Gelierung", Poster presented at PSI open 
doors, Paul Scherrer Institut, Villigen, Switzerland, 16th October, 2011.

- M. Cabanes-Sempere, C. Cozzo and M.A. Pouchon, "Advanced production of nuclear fuel particles", Poster presented at 494. Wilhelm und Else Heraeus-Seminar: Innovative Nuclear Power in a Closed Fuel Cycle Scenario, Bad Honnef, Germany, 5-8th December, 2011.

- C. Cozzo, M. Cabanes-Sempere and M.A. Pouchon, "Sphere-Pac fuel for advanced reactor systems", Poster presented at 494.Wilhelm und Else-Heraeus-Seminar: Innovative Nuclear Power in a Closed Fuel Cycle Scenario, Bad Honnef, Germany, 5-8th December, 2011.

- M. Cabanes-Sempere, "Characterization of Feed Solution Droplets for Nuclear Fuel Production inside a Microwave Cavity", Keynote at NES PhD Day 2012, Paul Scherrer Institut, Villigen, Switzerland, 13th June, 2012.

\section{Seminars and Courses}

- Seminar "Computer Modeling in Microwave Engineering $\&$ Applications", EMPA, Thun, Switzerland, 7-8th March, 2011.

- Grundlagenseminar "Einführung in die Kernenergie und ihr Umfeld", Kurszentrum Bundesamt für Sport, Magglingen, Switzerland, 19-21st April, 2011.

- Workshop 494. WE-Heraus-Seminar on "Innovative Nuclear Power in a Closed Fuel Cycle Scenario", Bad Honnef, Germany, 5-8th December, 2011.

- Course "Presentation Skills Course for Scientists", Laboratory of Inorganic Chemistry, ETH Hönggerberg, Zürich, Switzerland, 23rd May, 1st and 19th June, 2012.

- Workshop - Präsentations-Seminar "Auftreten und Reden" für Besucherführerinnen und Besucherführer des Paul Scherrer Institut (PSI), Zurzach/AG, Switzerland, 5th and 25-26 September, 2012. 
- Workshop "Permittivity and permeability measurements: Methods and Applications", Universitat Politècnica de València UPV, València, Spain, 10-18th September, 2012.

- Course "Advanced Presentation Training: Communication Skills Course", ETH Hönggerberg, Zürich, Switzerland, 28th November, 2012. 

Appendices

- 

Convective Heat Transfer Coefficient

A.1 Dimensionless groups in heat transfer . . . . . . . . 195

A.2 Properties of Air . . . . . . . . . . . . . . . . . 196 

Convective Heat Transfer Coefficient

\section{A.1 Dimensionless groups in heat transfer}

The convective heat transfer coefficient expressed in dimensionless form is known as Nusselt number, which is given as [48]:

$$
N_{u}=\frac{h_{T} D}{k_{T}}=\left.\frac{\partial T *}{\partial x^{*}}\right|_{x^{*}=0}
$$

where:

$$
\begin{aligned}
x^{*} & =\frac{x}{D} \\
T^{*} & =\frac{T-T_{S}}{T_{\infty}-T_{S}}
\end{aligned}
$$

are dimensionless variables referred to the characteristic length $(D)$ in case of $x^{*}$ and to the external free-stream condition $\left(T_{\infty}\right)$ and the conditions adjacent to a bounding surface across which heat and mass transfer occurs $\left(T_{S}\right)$ in case of $T^{*}$. 
The dimensionless temperature gradient at the surface also called Nusselt number, is related with other dimensionless parameters, i.e. the Reynolds number $R_{e}$ and the Prandtl number $P_{r}$. In the case of free falling liquid drops the Nusselt formula for the convective heat and mass transfer in the Reynolds number range $1 \leq R_{e} \leq 70.000$ reads [24, 92-95]

$$
N_{u}=2+0.6 R_{e}^{1 / 2} P_{r}^{1 / 3}
$$

The Reynolds number (a.k.a. non-dimensional velocity) is the parameter that represents the status of the flow and is defined as

$$
R_{e}=\frac{u D}{\nu}
$$

where $u$ is the velocity based on the actual cross section area of the duct or pipe $\left(\mathrm{m} \cdot \mathrm{s}^{-1}\right), D$ the diameter of the drop $(\mathrm{m})$ and $\nu$ the kinematic viscosity $\left(\mathrm{m}^{2} \cdot \mathrm{s}^{-1}\right)$.

In case $R_{e} \rightarrow 0$ the Nusselt number is reduced to $N_{u}=2$, which is the case of heat transfer by conduction in a spherical shell due to small velocity of the air relative to the droplet.

The Prandtl number represents the ratio of diffusion of momentum (given by the kinematic viscosity $\nu$ ) to diffusion of heat (given by the thermal diffusivity $\alpha$ ) in a fluid and is expressed as

$$
P_{r}=\frac{\nu}{\alpha}=\frac{\eta c_{p}}{k_{T}}
$$

where $\eta$ is the dynamic viscosity $\left(\mathrm{kg} \cdot \mathrm{m}^{-1} \cdot \mathrm{s}^{-1}\right), c_{p}$ the specific heat $\left(\mathrm{J} \cdot \mathrm{kg}^{-1} \cdot \mathrm{K}^{-1}\right)$ and $k_{T}$ the thermal conductivity $\left(\mathrm{W} \cdot \mathrm{m}^{-1} \cdot \mathrm{K}^{-1}\right)$.

\section{A.2 Properties of Air}

\section{A.2.1 Standard Properties}

The air is constituted of Nitrogen $\left(\mathrm{N}_{2}\right)$, Oxygen $\left(\mathrm{O}_{2}\right)$, Argon $(\mathrm{Ar})$ and Carbon dioxide $\left(\mathrm{CO}_{2}\right)$. Its properties are of interest when referring to its cooling effect during heat transfer. Table A.1 gives the basic values for standard conditions [96]. 


\section{A.2.2 Temperature Dependent Properties}

Convection situations do not always work with the same temperature conditions. Therefore the properties should be recalculated to adjust the models (see Tables A.2 and A.3). Most of the calculations use Kelvin as a unit, then the first step should be a temperature transformations as

$$
T_{K}=273.15+T_{C}
$$

in case of temperature in Celsius and

$$
T_{K}=273.15+\frac{\left(T_{F}-32\right)}{1.8}
$$

in case of temperature in Fahrenheit.

At absolute pressure the density in $\mathrm{kg} \cdot \mathrm{m}^{-3}$ is

$$
\rho=\frac{P}{R_{A} T_{K}}
$$

The specific thermal capacity in $\mathrm{J} \cdot \mathrm{kg}^{-1} \cdot \mathrm{K}^{-1}$ at constant pressure is given by

$$
c_{p}=1002.5+275 \times 10^{-6}\left(T_{K}-200\right)^{2}
$$

The thermal conductivity in $\mathrm{W} \cdot \mathrm{m}^{-1} \cdot \mathrm{K}^{-1}$ is

$$
k_{T}=\frac{0.02646 T_{K}^{1.5}}{T_{K}+245.4 \times 10^{-12 / T_{K}}}
$$

The dynamic viscosity in $\mathrm{kg} \cdot \mathrm{m}^{-1} \cdot \mathrm{s}^{-1}$ is given by

$$
\eta=\frac{0.1458 \times 10^{-5} T_{K}^{1.5}}{T_{K}+110.4}
$$

By definition, the kinematic viscosity in $\mathrm{m}^{2} \cdot \mathrm{s}^{-1}$ is

$$
\nu=\frac{\eta}{\rho}
$$

Table A.4 gives viscosity values for a temperature range between $100 \mathrm{~K}$ and $1600 \mathrm{~K}$. 
The Prandtl number is given by A.6, however a direct empirical expression in case of air is

$$
P_{r}=0.680+4.69 \times 10^{-7}\left(T_{K}-540\right)^{2}
$$

In practice, for normal air cooling $P_{r} \approx 0.7$. 


\begin{tabular}{llll}
\hline Property & Symbol & Value & Units \\
\hline Temperature & $T_{C}$ & 15 & ${ }^{\circ} \mathrm{C}$ \\
Pressure (absolute) & $T_{K}$ & 288.15 & $\mathrm{~K}$ \\
Density & $\rho$ & 101325 & $\mathrm{~Pa}$ \\
Dynamic viscosity & $\eta$ & 1.2256 & $\mathrm{~kg} \cdot \mathrm{m}^{-3}$ \\
Kinematic viscosity & $\nu$ & $1.783 \times 10^{-5}$ & $\mathrm{~kg} \cdot \mathrm{m}^{-1} \cdot \mathrm{s}^{-1}$ \\
Specific gas constant & $R_{A}$ & 287.05 & $\mathrm{~J} \cdot \mathrm{kg}^{-1} \cdot \mathrm{K}^{-1}$ \\
Specific heat & $c_{p}$ & 1005 & $\mathrm{~J} \cdot \mathrm{kg}^{-1} \cdot \mathrm{K}^{-1}$ \\
Thermal conductivity & $k_{T}$ & 0.02534 & $\mathrm{~W} \cdot \mathrm{m}^{-1} \cdot \mathrm{K}^{-1}$ \\
Prandtl number & $P_{r}$ & 0.710 & - \\
\hline
\end{tabular}

Table A.1. Standard properties of dry air at sea-level. 


\begin{tabular}{cccc}
\hline Temperature & Density & $\begin{array}{c}\text { Specific } \\
\text { heat capacity }\end{array}$ & $\begin{array}{c}\text { Thermal } \\
\text { conductivity }\end{array}$ \\
$\mathrm{T}\left({ }^{\circ} \mathrm{C}\right)$ & $\rho\left(\mathrm{kg} \cdot \mathrm{m}^{-3}\right)$ & $c_{p}\left(\mathrm{~J} \cdot \mathrm{kg}^{-1} \cdot \mathrm{K}^{-1}\right)$ & $k_{T}\left(\mathrm{~W} \cdot \mathrm{m}^{-1} \cdot \mathrm{K}^{-1}\right)$ \\
\hline-150 & 2.793 & 1.026 & 0.0116 \\
-100 & 1.980 & 1.009 & 0.0160 \\
-50 & 1.534 & 1.005 & 0.0204 \\
0 & 1.293 & 1.005 & 0.0243 \\
20 & 1.205 & 1.005 & 0.0257 \\
40 & 1.127 & 1.005 & 0.0271 \\
60 & 1.067 & 1.009 & 0.0285 \\
80 & 1.000 & 1.009 & 0.0299 \\
100 & 0.946 & 1.009 & 0.0314 \\
120 & 0.898 & 1.013 & 0.0328 \\
140 & 0.854 & 1.013 & 0.0343 \\
160 & 0.815 & 1.017 & 0.0358 \\
180 & 0.779 & 1.022 & 0.0372 \\
200 & 0.746 & 1.026 & 0.0386 \\
250 & 0.675 & 1.034 & 0.0421 \\
300 & 0.616 & 1.047 & 0.0454 \\
350 & 0.566 & 1.055 & 0.0485 \\
400 & 0.524 & 1.068 & 0.0515 \\
\hline
\end{tabular}

Table A.2. Common properties for air at atmospheric pressure I. 


\begin{tabular}{ccc}
\hline Temperature & $\begin{array}{c}\text { Kinematic } \\
\text { viscosity } \\
\mathrm{T}\left(\mathrm{m}^{\circ} \mathrm{C}\right)\end{array}$ & $\begin{array}{c}\text { Prandtl's } \\
\text { number }\end{array}$ \\
\hline-150 & 3.08 & $P_{r}(-)$ \\
-100 & 5.95 & 0.76 \\
-50 & 9.55 & 0.74 \\
0 & 13.30 & 0.725 \\
20 & 15.11 & 0.715 \\
40 & 16.97 & 0.713 \\
60 & 18.90 & 0.711 \\
80 & 20.94 & 0.709 \\
100 & 23.06 & 0.708 \\
120 & 25.23 & 0.703 \\
140 & 27.55 & 0.70 \\
160 & 29.85 & 0.695 \\
180 & 32.29 & 0.69 \\
200 & 34.63 & 0.69 \\
250 & 41.17 & 0.685 \\
300 & 47.85 & 0.68 \\
350 & 55.05 & 0.68 \\
400 & 62.53 & 0.68 \\
\hline
\end{tabular}

Table A.3. Common properties for air at atmospheric pressure II. 


\begin{tabular}{ccc}
\hline $\begin{array}{c}\text { Temperature } \\
T_{K}(\mathrm{~K})\end{array}$ & $\begin{array}{c}\text { Dynamic Viscosity } \\
\eta\left(\mathrm{kg} \cdot \mathrm{m}^{-1} \cdot \mathrm{s}^{-1}\right) \times 10^{-5}\end{array}$ & $\begin{array}{c}\text { Kinematic Viscosity } \\
\nu\left(\mathrm{m}^{2} \cdot \mathrm{s}^{-1}\right) \times 10^{-6}\end{array}$ \\
\hline 100 & 0.6924 & 1.923 \\
150 & 1.0283 & 4.343 \\
200 & 1.3289 & 7.490 \\
250 & 1.488 & 9.49 \\
300 & 1.983 & 15.68 \\
350 & 2.075 & 20.76 \\
400 & 2.286 & 25.90 \\
450 & 2.484 & 28.86 \\
500 & 2.671 & 37.90 \\
550 & 2.848 & 44.34 \\
600 & 3.018 & 51.34 \\
650 & 3.177 & 58.51 \\
700 & 3.332 & 66.25 \\
752 & 3.481 & 73.91 \\
800 & 3.625 & 82.29 \\
850 & 3.765 & 90.75 \\
900 & 3.899 & 99.30 \\
950 & 4.023 & 108.2 \\
1000 & 4.152 & 117.8 \\
1100 & 4.44 & 138.6 \\
1200 & 4.69 & 159.1 \\
1300 & 4.93 & 182.1 \\
1400 & 5.17 & 205.5 \\
1500 & 5.40 & 229.1 \\
1600 & 5.63 & 254.5 \\
\hline
\end{tabular}

Table A.4. Absolute and kinematic viscosity of air at standard atmospheric pressure - SI units. 


\section{Crank Nicholson Development}

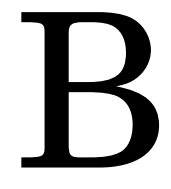

B.1 Rectangular Coordinates . . . . . . . . . . . . . . 207

B.2 Cylindrical Coordinates . . . . . . . . . . . . . 208

B.3 Spherical Coordinates . . . . . . . . . . . . . . . 209

B.4 General equation . . . . . . . . . . . . . . . . . . . . 211 



\section{Crank Nicholson Development}

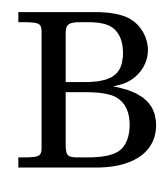

Partial differential equations applied to diffusion problems, are discretized by using the Crank-Nicholson strategy [51]. As a difference with respect to the Euler method (dependent on the backward or forward time), this strategy is a second order method in time based on central difference in space. The forward Euler method at $n$ is given by:

$$
\frac{u_{i}^{n+1}-u_{i}^{n}}{\Delta t}=F_{i}^{n}\left(u, x, t, \frac{\partial u}{\partial x}, \frac{\partial^{2} u}{\partial x^{2}}\right)
$$

and the backward Euler method at $n+1$ is:

$$
\frac{u_{i}^{n+1}-u_{i}^{n}}{\Delta t}=F_{i}^{n+1}\left(u, x, t, \frac{\partial u}{\partial x}, \frac{\partial^{2} u}{\partial x^{2}}\right)
$$

Finally the Crank-Nicholson method is formulated as:

$$
\frac{u_{i}^{n+1}-u_{i}^{n}}{\Delta t}=\frac{1}{2}\left[F_{i}^{n+1}\left(u, x, t, \frac{\partial u}{\partial x}, \frac{\partial^{2} u}{\partial x^{2}}\right)+F_{i}^{n}\left(u, x, t, \frac{\partial u}{\partial x}, \frac{\partial^{2} u}{\partial x^{2}}\right)\right]
$$

Over the same time step $n+1$, based on the representation of Figure B.1, the first order spatial derivative is computed from two neighbour 


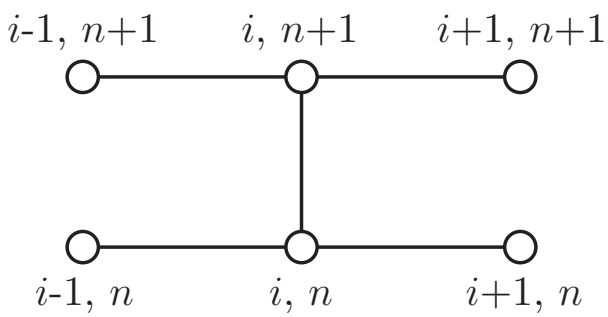

Figure B.1. Crank Nicholson scheme for a one dimensional approach.

nodes as:

$$
\frac{\partial u}{\partial x}=\frac{u_{i+1}^{n+1}-u_{i}^{n+1}}{\Delta x}
$$

In case of keeping the same spatial node $i$, the first order time partial derivative is calculated by a linear interpolation of the adjacent time steps as:

$$
\frac{\partial u}{\partial t}=\frac{u_{i}^{n+1}-u_{i}^{n}}{\Delta t}
$$

Diffusion problems depend also on the second order spatial derivative. To calculate the value of the second order spatial derivative a Taylor expansion up to the second order is applied to the function $u(x, t)$ in $x_{i}+\Delta x$ and $x_{i}-\Delta x$.

$$
\begin{aligned}
& u\left(x_{i}, t_{n}\right)=u_{i}^{n} \\
& u_{i+1}^{n}=u_{i}^{n}+\left.\Delta x \frac{\partial u}{\partial x}\right|_{i, n}+\left.\frac{\Delta x^{2}}{2} \frac{\partial^{2} u}{\partial x^{2}}\right|_{i, n}+\vartheta\left(\Delta x^{2}\right) \\
& u_{i-1}^{n}=u_{i}^{n}-\left.\Delta x \frac{\partial u}{\partial x}\right|_{i, n}+\left.\frac{\Delta x^{2}}{2} \frac{\partial^{2} u}{\partial x^{2}}\right|_{i, n}+\vartheta\left(\Delta x^{2}\right)
\end{aligned}
$$

The sum of B.7 and B.8 leads to the second order spatial derivative as:

$$
\left.\frac{\partial^{2} u}{\partial x^{2}}\right|_{x_{i}, t_{n}}=\left[\frac{u\left(x_{i}+\Delta x\right)-2 u\left(x_{i}\right)+u\left(x_{i}-\Delta x\right)}{\Delta x^{2}}\right]_{t_{n}}+\vartheta\left(\Delta x^{2}\right)
$$


or in compact form:

$$
\left.\frac{\partial^{2} u}{\partial x^{2}}\right|_{x_{i}, t_{n}}=\frac{u_{i+1}^{n}-2 u_{i}^{n}+u_{i-1}^{n}}{\Delta x^{2}}+\vartheta\left(\Delta x^{2}\right)
$$

where the time coordinate is fixed while the space coordinate changes.

If the time dependence is enabled between two continuous instants $(n$ and $n+1)$, then:

$$
\frac{\partial^{2} u}{\partial x^{2}}=\frac{1}{2}\left[\frac{u_{i-1}^{n+1}-2 u_{i}^{n+1}+u_{i+1}^{n+1}}{\Delta x^{2}}+\frac{u_{i-1}^{n}-2 u_{i}^{n}+u_{i+1}^{n}}{\Delta x^{2}}\right]
$$

which is the Crank-Nicholson formulation used in diffusion problems such as the heat equation.

Over the next sections the development of the heat equations shown in Chapter 3 are presented.

\section{B.1 Rectangular Coordinates}

One-dimensional heat equation in rectangular coordinates.

$$
\frac{\partial T}{\partial t}=\left(\frac{k_{T}}{\rho c_{p}}\right) \frac{\partial^{2} T}{\partial z^{2}}+\frac{1}{\rho c_{p}} Q_{g e n}
$$

Application of B.5 and B.11.

$$
\begin{aligned}
& \frac{T_{i}^{n+1}-T_{i}^{n}}{\Delta t}=\alpha \frac{1}{2}\left[\frac{T_{i-1}^{n+1}-2 T_{i}^{n+1}+T_{i+1}^{n+1}}{\Delta z^{2}}+\frac{T_{i-1}^{n}-2 T_{i}^{n}+T_{i+1}^{n}}{\Delta z^{2}}\right]+\beta g_{i}^{n} \\
& T_{i}^{n+1}-T_{i}^{n}=\frac{\alpha \Delta t}{\Delta z^{2}} \frac{1}{2}\left[\left(T_{i-1}^{n+1}-2 T_{i}^{n+1}+T_{i+1}^{n+1}\right)+\left(T_{i-1}^{n}-2 T_{i}^{n}+T_{i+1}^{n}\right)\right]+\Delta t \beta g_{i}^{n}
\end{aligned}
$$

Considering $\mathbf{s}^{n}=\Delta t \beta \mathbf{g}^{n}$ and $c_{1}=\frac{\alpha \Delta t}{\Delta z^{2}}$ :

$$
T_{i}^{n+1}-\frac{1}{2} c_{1}\left[T_{i-1}^{n+1}-2 T_{i}^{n+1}+T_{i+1}^{n+1}\right]=T_{i}^{n}+\frac{1}{2} c_{1}\left[T_{i-1}^{n}-2 T_{i}^{n}+T_{i+1}^{n}\right]+\mathbf{s}^{n}
$$


Considering

$$
\mathbf{A}=\left(\begin{array}{ccccc}
-2 & 1 & 0 & \cdots & 0 \\
1 & -2 & 1 & \ddots & \vdots \\
0 & \vdots & \vdots & \vdots & 0 \\
\vdots & \ddots & 1 & -2 & 1 \\
0 & \cdots & 0 & 1 & -2
\end{array}\right)
$$

and

$$
\mathbf{t}^{n}=\left(\begin{array}{c}
T_{1}^{n} \\
T_{2}^{n} \\
\vdots \\
T_{N-1}^{n} \\
T_{N}^{n}
\end{array}\right) \mathbf{t}^{n+1}=\left(\begin{array}{c}
T_{1}^{n+1} \\
T_{2}^{n+1} \\
\vdots \\
T_{N-1}^{n+1} \\
T_{N}^{n+1}
\end{array}\right) \mathbf{b}^{n}=\left(\begin{array}{c}
b_{0}^{n} \\
0 \\
\vdots \\
0 \\
b_{N+1}^{n}
\end{array}\right) \mathbf{g}^{n}=\left(\begin{array}{c}
g_{1}^{n} \\
g_{2}^{n} \\
\vdots \\
g_{N-1}^{n} \\
g_{N}^{n}
\end{array}\right)
$$

the final equation is obtained:

$$
\left(\mathbf{I}-\frac{1}{2} c_{1} \mathbf{A}\right) \mathbf{t}^{n+1}=\left(\mathbf{I}+\frac{1}{2} c_{1} \mathbf{A}\right) \mathbf{t}^{n}+\frac{c_{1}}{2}\left(\mathbf{b}^{n+1}+\mathbf{b}^{n}\right)+\mathbf{s}^{n}
$$

\section{B.2 Cylindrical Coordinates}

One-dimensional heat equation in cylindrical coordinates.

$$
\frac{\partial T}{\partial t}=\left(\frac{k_{T}}{\rho c_{p}}\right) \frac{1}{r} \frac{\partial}{\partial r}\left(r \frac{\partial T}{\partial r}\right)+\frac{1}{\rho c_{p}} Q_{g e n}
$$

Derivation:

$$
\frac{\partial T}{\partial t}=\left(\frac{k_{T}}{\rho c_{p}}\right) \frac{1}{r}\left(\frac{\partial T}{\partial r}+r \frac{\partial^{2} T}{\partial r^{2}}\right)+\frac{1}{\rho c_{p}} Q_{g e n}
$$

Application of B.4, B.5 and B.11.

$$
\begin{aligned}
& \frac{T_{i}^{n+1}-T_{i}^{n}}{\Delta t}=\alpha \frac{1}{2}\left[\frac{1}{r} \frac{T_{i+1}^{n+1}-T_{i-1}^{n+1}}{\Delta r}+\frac{T_{i-1}^{n+1}-2 T_{i}^{n+1}+T_{i+1}^{n+1}}{\Delta r^{2}}\right. \\
& \left.+\frac{T_{i-1}^{n}-2 T_{i}^{n}+T_{i+1}^{n}}{\Delta r^{2}}\right]+\beta g_{i}^{n}
\end{aligned}
$$


Considering $\mathbf{s}^{n}=\Delta t \beta \mathbf{g}^{n}, c_{1}=\frac{\alpha \Delta t}{\Delta r^{2}}$ and $c_{2}=\frac{\alpha \Delta t}{\Delta r} \frac{1}{r}$ :

$$
\begin{aligned}
T_{i}^{n+1} & -\frac{1}{2} c_{1}\left(T_{i-1}^{n+1}-2 T_{i}^{n+1}+T_{i+1}^{n+1}\right)-\frac{1}{2} c_{2}\left(T_{i+1}^{n+1}-T_{i-1}^{n+1}\right)= \\
T_{i}^{n} & +\frac{1}{2} c_{1}\left(T_{i-1}^{n}-2 T_{i}^{n}+T_{i+1}^{n}\right)+\mathbf{s}^{n}
\end{aligned}
$$

Considering

$$
\mathbf{A}=\left(\begin{array}{ccccc}
-2 & 1 & 0 & \cdots & 0 \\
1 & -2 & 1 & \ddots & \vdots \\
0 & \vdots & \vdots & \vdots & 0 \\
\vdots & \ddots & 1 & -2 & 1 \\
0 & \cdots & 0 & 1 & -2
\end{array}\right) \quad \mathbf{B}=\left(\begin{array}{ccccc}
0 & 1 & 0 & \cdots & 0 \\
-1 & 0 & 1 & \ddots & \vdots \\
0 & \vdots & \vdots & \vdots & 0 \\
\vdots & \ddots & -1 & 0 & 1 \\
0 & \cdots & 0 & -1 & 0
\end{array}\right)
$$

and

$$
\mathbf{t}^{n}=\left(\begin{array}{c}
T_{1}^{n} \\
T_{2}^{n} \\
\vdots \\
T_{N-1}^{n} \\
T_{N}^{n}
\end{array}\right) \mathbf{t}^{n+1}=\left(\begin{array}{c}
T_{1}^{n+1} \\
T_{2}^{n+1} \\
\vdots \\
T_{N-1}^{n+1} \\
T_{N}^{n+1}
\end{array}\right) \mathbf{b}^{n}=\left(\begin{array}{c}
b_{0}^{n} \\
0 \\
\vdots \\
0 \\
b_{N+1}^{n}
\end{array}\right) \mathbf{g}^{n}=\left(\begin{array}{c}
g_{1}^{n} \\
g_{2}^{n} \\
\vdots \\
g_{N-1}^{n} \\
g_{N}^{n}
\end{array}\right)
$$

the final equation is obtained:

$$
\left(\mathbf{I}-\frac{1}{2} c_{1} \mathbf{A}-\frac{1}{2} c_{2} \mathbf{B}\right) \mathbf{t}^{n+1}=\left(\mathbf{I}+\frac{1}{2} c_{1} \mathbf{A}\right) \mathbf{t}^{n}+\frac{c_{1}}{2}\left(\mathbf{b}^{n+1}+\mathbf{b}^{n}\right)+\mathbf{s}^{n}
$$

\section{B.3 Spherical Coordinates}

One-dimensional heat equation in spherical coordinates.

$$
\frac{\partial T}{\partial t}=\left(\frac{k_{T}}{\rho c_{p}}\right) \frac{1}{r^{2}} \frac{\partial}{\partial r}\left(r^{2} \frac{\partial T}{\partial r}\right)+\frac{1}{\rho c_{p}} Q_{g e n}
$$

Derivation:

$$
\frac{\partial T}{\partial t}=\left(\frac{k_{T}}{\rho c_{p}}\right) \frac{1}{r^{2}}\left(2 r \frac{\partial T}{\partial r}+r^{2} \frac{\partial^{2} T}{\partial r^{2}}\right)+\frac{1}{\rho c_{p}} Q_{g e n}
$$


Application of B.4, B.5 and B.11.

$$
\begin{aligned}
& \frac{T_{i}^{n+1}-T_{i}^{n}}{\Delta t}=\alpha\left[\frac{1}{r} \frac{T_{i+1}^{n+1}-T_{i-1}^{n+1}}{\Delta r}+\frac{1}{2}\left(\frac{T_{i-1}^{n+1}-2 T_{i}^{n+1}+T_{i+1}^{n+1}}{\Delta r^{2}}\right.\right. \\
& \left.\left.+\frac{T_{i-1}^{n}-2 T_{i}^{n}+T_{i+1}^{n}}{\Delta r^{2}}\right)\right]+\beta g_{i}^{n}
\end{aligned}
$$

Considering $\mathbf{s}^{n}=\Delta t \beta \mathbf{g}^{n}, c_{1}=\frac{\alpha \Delta t}{\Delta r^{2}}$ and $c_{2}=\frac{\alpha \Delta t}{\Delta r} \frac{2}{r}$ :

$$
\begin{aligned}
T_{i}^{n+1} & -\frac{1}{2} c_{1}\left(T_{i-1}^{n+1}-2 T_{i}^{n+1}+T_{i+1}^{n+1}\right)-\frac{1}{2} c_{2}\left(T_{i+1}^{n+1}-T_{i-1}^{n+1}\right)= \\
T_{i}^{n} & +\frac{1}{2} c_{1}\left(T_{i-1}^{n}-2 T_{i}^{n}+T_{i+1}^{n}\right)+\mathbf{s}^{n}
\end{aligned}
$$

Considering:

$$
\mathbf{A}=\left(\begin{array}{ccccc}
-2 & 1 & 0 & \cdots & 0 \\
1 & -2 & 1 & \ddots & \vdots \\
0 & \vdots & \vdots & \vdots & 0 \\
\vdots & \ddots & 1 & -2 & 1 \\
0 & \cdots & 0 & 1 & -2
\end{array}\right) \quad \mathbf{B}=\left(\begin{array}{ccccc}
0 & 1 & 0 & \cdots & 0 \\
-1 & 0 & 1 & \ddots & \vdots \\
0 & \vdots & \vdots & \vdots & 0 \\
\vdots & \ddots & -1 & 0 & 1 \\
0 & \cdots & 0 & -1 & 0
\end{array}\right)
$$

and

$$
\mathbf{t}^{n}=\left(\begin{array}{c}
T_{1}^{n} \\
T_{2}^{n} \\
\vdots \\
T_{N-1}^{n} \\
T_{N}^{n}
\end{array}\right) \mathbf{t}^{n+1}=\left(\begin{array}{c}
T_{1}^{n+1} \\
T_{2}^{n+1} \\
\vdots \\
T_{N-1}^{n+1} \\
T_{N}^{n+1}
\end{array}\right) \mathbf{b}^{n}=\left(\begin{array}{c}
b_{0}^{n} \\
0 \\
\vdots \\
0 \\
b_{N+1}^{n}
\end{array}\right) \mathbf{g}^{n}=\left(\begin{array}{c}
g_{1}^{n} \\
g_{2}^{n} \\
\vdots \\
g_{N-1}^{n} \\
g_{N}^{n}
\end{array}\right)
$$

the final equation is obtained:

$$
\left(\mathbf{I}-\frac{1}{2} c_{1} \mathbf{A}-\frac{1}{2} c_{2} \mathbf{B}\right) \mathbf{t}^{n+1}=\left(\mathbf{I}+\frac{1}{2} c_{1} \mathbf{A}\right) \mathbf{t}^{n}+\frac{c_{1}}{2}\left(\mathbf{b}^{n+1}+\mathbf{b}^{n}\right)+\mathbf{s}^{n}
$$




\section{B.4 General equation}

The three coordinate system equations follow the same structure differing only in the term accompanied by the $c_{2}$ constant:

$$
\left(\mathbf{I}-\frac{1}{2} c_{1} \mathbf{A}-\frac{1}{2} c_{2} \mathbf{B}\right) \mathbf{t}^{n+1}=\left(\mathbf{I}+\frac{1}{2} c_{1} \mathbf{A}\right) \mathbf{t}^{n}+\frac{c_{1}}{2}\left(\mathbf{b}^{n+1}+\mathbf{b}^{n}\right)+\mathbf{s}^{n}
$$

where the constants, matrices and vectors are defined as follows:

$$
\begin{aligned}
& \mathbf{s}^{n}=\Delta t \beta \mathbf{g}^{n}, \\
& c_{1}=\frac{\alpha \Delta t}{\Delta z^{2}}, c_{2}=\frac{\alpha \Delta t}{\Delta r} \frac{1}{r}(\delta-1)\left\{\begin{array}{l}
\delta=1 \text { (rectangular coordinates) } \\
\delta=2 \text { (cylindrical coordinates) } \\
\delta=3 \text { (spherical coordinates) }
\end{array}\right. \\
& \mathbf{A}=\left(\begin{array}{ccccc}
-2 & 1 & 0 & \cdots & 0 \\
1 & -2 & 1 & \ddots & \vdots \\
0 & \vdots & \vdots & \vdots & 0 \\
\vdots & \ddots & 1 & -2 & 1 \\
0 & \cdots & 0 & 1 & -2
\end{array}\right) \quad \mathbf{B}=\left(\begin{array}{ccccc}
0 & 1 & 0 & \cdots & 0 \\
-1 & 0 & 1 & \ddots & \vdots \\
0 & \vdots & \vdots & \vdots & 0 \\
\vdots & \ddots & -1 & 0 & 1 \\
0 & \cdots & 0 & -1 & 0
\end{array}\right) \\
& \mathbf{t}^{n}=\left(\begin{array}{c}
T_{1}^{n} \\
T_{2}^{n} \\
\vdots \\
T_{N-1}^{n} \\
T_{N}^{n}
\end{array}\right) \mathbf{t}^{n+1}=\left(\begin{array}{c}
T_{1}^{n+1} \\
T_{2}^{n+1} \\
\vdots \\
T_{N-1}^{n+1} \\
T_{N}^{n+1}
\end{array}\right) \mathbf{b}^{n}=\left(\begin{array}{c}
b_{0}^{n} \\
0 \\
\vdots \\
0 \\
b_{N+1}^{n}
\end{array}\right) \mathbf{g}^{n}=\left(\begin{array}{c}
g_{1}^{n} \\
g_{2}^{n} \\
\vdots \\
g_{N-1}^{n} \\
g_{N}^{n}
\end{array}\right)
\end{aligned}
$$

Depending on which boundary conditions of the material are applied to the study, the matrix $\mathbf{A}$ and the vector $\mathbf{b}$ will be changed to adjust the model to these conditions (see 3.4 and $\mathrm{A}$ ). The vector $\mathbf{g}$ changes depending on the features of the microwaves cavity and the heat generation rate $Q_{g e n}$. 



\section{Energy and Power Solutions}

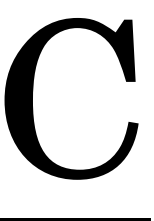

C.1 Calculation of Energies in a Rectangular Empty Cavity . 215

C.2 Calculation of Power in a Rectangular Empty Cavity . . . 219 



\section{Energy and Power Solutions}

\section{C.1 Calculation of Energies in a Rectangular Empty Cavity}

The stored average electric energy is defined as

$$
W_{e}=\frac{\varepsilon_{0}}{4} \int_{V_{c}}|\vec{E}|^{2} d v
$$

The field Equations 4.13 and 4.14 are applied to solve the $W_{e}$ expression. In the case of $\mathrm{TE}_{m n p}$ modes:

$$
\begin{aligned}
W_{e} & =\frac{\varepsilon_{0}}{4} \int_{V_{c}} A_{m n}^{2} \frac{\left(\omega \mu_{0}\right)^{2}}{k_{c, m n}^{4}}\left[\frac{n \pi}{b} \cos \left(\frac{m \pi}{a} x\right) \sin \left(\frac{n \pi}{b} y\right) \sin \left(\frac{p \pi}{L} z\right)\right. \\
& \left.+\frac{m \pi}{a} \sin \left(\frac{m \pi}{a} x\right) \cos \left(\frac{n \pi}{b} y\right) \sin \left(\frac{p \pi}{L} z\right)\right]^{2} d V
\end{aligned}
$$

Considering:

$$
(a+b)^{2}=a^{2}+b^{2}+2 a b
$$




$$
\begin{aligned}
W_{e} & =\frac{\varepsilon_{0}}{4} A_{m n}^{2} \frac{\left(\omega \mu_{0}\right)^{2}}{k_{c, m n}^{4}} \int_{0}^{L} \sin ^{2}\left(\frac{p \pi}{L} z\right) d z\left\{\left(\frac{n \pi}{b}\right)^{2} \int_{0}^{a} \cos ^{2}\left(\frac{m \pi}{a} x\right) d x \int_{0}^{b} \sin ^{2}\left(\frac{n \pi}{b} y\right) d y\right. \\
& +\left(\frac{m \pi}{a}\right)^{2} \int_{0}^{a} \sin ^{2}\left(\frac{m \pi}{a} x\right) d x \int_{0}^{b} \cos ^{2}\left(\frac{n \pi}{b} y\right) d y \\
& \left.+2 \frac{n \pi}{b} \frac{m \pi}{a} \int_{0}^{a} \sin \left(\frac{m \pi}{a} x\right) \cos \left(\frac{m \pi}{a} x\right) d x \int_{0}^{b} \sin \left(\frac{n \pi}{b} y\right) \cos \left(\frac{n \pi}{b} y\right) d y\right\}
\end{aligned}
$$

Considering the following developments:

$$
\begin{aligned}
& \int_{0}^{a} \sin ^{2}\left(\frac{m \pi}{a} x\right) d x=\int_{0}^{a} \frac{1}{2}\left(1-\cos \left(\frac{2 m \pi}{a} x\right)\right) d x \\
& =\frac{1}{2}\left[x-\frac{a}{2 m \pi} \sin \left(\frac{2 m \pi}{a} x\right)\right]_{0}^{a} \\
& =\frac{a}{2}-\frac{a}{4 m \pi} \sin (2 m \pi)\left\{\begin{array}{ll}
m=0 & \int_{0}^{a} \sin ^{2} 0 d x=0 \\
m \neq 0 & +\frac{a}{2}
\end{array}\right\}=\frac{a}{2} \varepsilon_{m(\sin )} \\
& \int_{0}^{a} \cos ^{2}\left(\frac{m \pi}{a} x\right) d x=\int_{0}^{a} \frac{1}{2}\left(1+\cos \left(\frac{2 m \pi}{a} x\right)\right) d x \\
& =\frac{1}{2}\left[x+\frac{a}{2 m \pi} \sin \left(\frac{2 m \pi}{a} x\right)\right]_{0}^{a} \begin{array}{ll}
m \\
m=0 & \int_{0}^{a} \cos ^{2} 0 d x=a \\
m \neq 0 & +\frac{a}{2}
\end{array}=\frac{a}{2} \varepsilon_{m(\cos )} \\
& =\frac{a}{2}+\frac{a}{4 m \pi} \sin (2 m \pi)\left\{\begin{array}{l}
m \\
m
\end{array}\right)
\end{aligned}
$$

which introduce the constants $\varepsilon_{m}, \varepsilon_{n}$ and $\varepsilon_{p}$ as

$$
\begin{aligned}
& \varepsilon_{m, n, p(\sin )}= \begin{cases}0 & m, n, p=0 \\
1 & m, n, p \neq 0\end{cases} \\
& \varepsilon_{m, n, p(\cos )}= \begin{cases}2 & m, n, p=0 \\
1 & m, n, p \neq 0\end{cases}
\end{aligned}
$$

$\varepsilon_{m(\sin )}, \varepsilon_{n(\sin )}$ and $\varepsilon_{p(\sin )}$ appear always together with $m, n$ and $p$, respectively. For these reason they are simplified and do not appear on the final equations. Other simplification is:

$$
\int_{0}^{a} \sin \left(\frac{m \pi}{a} x\right) \cos \left(\frac{m \pi}{a} x\right) d x=\frac{a}{m \pi} \frac{1}{2}\left[\sin ^{2}\left(\frac{m \pi}{a} x\right)\right]_{0}^{a}=0
$$

The electric energy equation is finally:

$$
W_{e}=\frac{\varepsilon_{0}}{4} \frac{A_{m n}^{2} \omega^{2} \mu_{0}^{2}}{k_{c, m n}^{2}} \frac{a b L}{8} \varepsilon_{m} \varepsilon_{n}
$$


with:

$$
\begin{gathered}
\varepsilon_{m}= \begin{cases}2, & m=0 \\
1, & m \neq 0\end{cases} \\
\varepsilon_{n}= \begin{cases}2, & n=0 \\
1, & n \neq 0\end{cases}
\end{gathered}
$$

In the case of $\mathrm{TM}_{m n p}$ modes:

$$
\begin{aligned}
W_{e} & =\frac{\varepsilon_{0}}{4} \int_{V_{c}}\left[B_{m n} \frac{\beta_{z}}{k_{c, m n}^{2}} \frac{m \pi}{a} \cos \left(\frac{m \pi}{a} x\right) \sin \left(\frac{n \pi}{b} y\right) \sin \left(\frac{p \pi}{L} z\right)\right. \\
- & B_{m n} \frac{\beta_{z}}{k_{c, m n}^{2}} \frac{n \pi}{b} \sin \left(\frac{m \pi}{a} x\right) \cos \left(\frac{n \pi}{b} y\right) \sin \left(\frac{p \pi}{L} z\right) \\
+ & \left.B_{m n} \sin \left(\frac{m \pi}{a} x\right) \sin \left(\frac{n \pi}{b} y\right) \cos \left(\frac{p \pi}{L} z\right)\right]^{2} d V
\end{aligned}
$$

Considering:

$$
(a+b+c)^{2}=a^{2}+b^{2}+c^{2}+2 a b+2 a c+2 b c
$$

then,

$$
\begin{aligned}
W_{e} & =\frac{\varepsilon_{0}}{4} B_{m n}^{2}\left\{\frac { \beta _ { z } ^ { 2 } } { k _ { c , m n } ^ { 4 } } \int _ { 0 } ^ { L } \operatorname { s i n } ^ { 2 } ( \frac { p \pi } { L } z ) d z \left\{\left(\frac{m \pi}{a}\right)^{2} \int_{0}^{a} \cos ^{2}\left(\frac{m \pi}{a} x\right) d x \int_{0}^{b} \sin ^{2}\left(\frac{n \pi}{b} y\right) d y\right.\right. \\
& +\left(\frac{n \pi}{b}\right)^{2} \int_{0}^{a} \sin ^{2}\left(\frac{m \pi}{a} x\right) d x \int_{0}^{b} \cos ^{2}\left(\frac{n \pi}{b} y\right) d y \\
& \left.+2 \frac{m \pi}{a} \frac{n \pi}{b} \int_{0}^{a} \sin \left(\frac{m \pi}{a} x\right) \cos \left(\frac{m \pi}{a} x\right) d x \int_{0}^{b} \sin \left(\frac{n \pi}{b} y\right) \cos \left(\frac{n \pi}{b} y\right) d y\right\} \\
& -\frac{\beta_{z}}{k_{c, m n}^{2}} \int_{0}^{L} \sin \left(\frac{p \pi}{L} z\right) \cos \left(\frac{p \pi}{L} z\right) d z\left\{2 \frac{m \pi}{a} \int_{0}^{a} \sin \left(\frac{m \pi}{a} x\right) \cos \left(\frac{m \pi}{a} x\right) d x \int_{0}^{b} \sin ^{2}\left(\frac{n \pi}{b} y\right) d y\right. \\
& \left.+2 \frac{n \pi}{b} \int_{0}^{a} \sin ^{2}\left(\frac{m \pi}{a} x\right) d x \int_{0}^{b} \sin \left(\frac{n \pi}{b} y\right) \cos \left(\frac{n \pi}{b} y\right) d y\right\} \\
& \left.+\int_{0}^{a} \sin ^{2}\left(\frac{m \pi}{a} x\right) d x \int_{0}^{b} \sin ^{2}\left(\frac{n \pi}{b} y\right) d y \int_{0}^{L} \cos ^{2}\left(\frac{p \pi}{L} z\right) d z\right\}
\end{aligned}
$$

Considering Equations C.3, C.4 and C.7 the electric energy equation for the $\mathrm{TM}_{m n p}$ modes is finally:

$$
W_{e}=\frac{\varepsilon_{0}}{4} B_{m n}^{2} \frac{a b L}{8} \varepsilon_{p}\left(1+\frac{\beta_{z}^{2}}{k_{c, m n}^{2}}\right)
$$

with:

$$
\varepsilon_{p}= \begin{cases}2, & p=0 \\ 1, & p \neq 0\end{cases}
$$


The stored average magnetic energy is defined as

$$
W_{m}=\frac{\mu_{0}}{4} \int_{V_{c}}|\vec{H}|^{2} d v
$$

The field Equations 4.13 and 4.14 are applied to solve the $W_{m}$ expression. In the case of $\mathrm{TE}_{m n p}$ modes:

$$
\begin{gathered}
W_{m}=-\frac{\mu_{0}}{4} \int_{V_{c}}\left[A_{m n} \frac{\beta_{z}}{k_{c, m n}^{2}} \frac{m \pi}{a} \sin \left(\frac{m \pi}{a} x\right) \cos \left(\frac{n \pi}{b} y\right) \cos \left(\frac{p \pi}{L} z\right)\right. \\
+\quad A_{m n} \frac{\beta_{z}}{k_{c, m n}^{2}} \frac{n \pi}{b} \cos \left(\frac{m \pi}{a} x\right) \sin \left(\frac{n \pi}{b} y\right) \cos \left(\frac{p \pi}{L} z\right) \\
\left.-\quad A_{m n} \cos \left(\frac{m \pi}{a} x\right) \cos \left(\frac{n \pi}{b} y\right) \sin \left(\frac{p \pi}{L} z\right)\right]^{2} d V
\end{gathered}
$$

Considering Equation C.9

$$
\begin{aligned}
W_{m} & =-\frac{\mu_{0}}{4} A_{m n}^{2}\left\{\frac { \beta _ { z } ^ { 2 } } { k _ { c , m n } ^ { 4 } } \int _ { 0 } ^ { L } \operatorname { c o s } ^ { 2 } ( \frac { p \pi } { L } z ) d z \left\{\left(\frac{m \pi}{a}\right)^{2} \int_{0}^{a} \sin ^{2}\left(\frac{m \pi}{a} x\right) d x \int_{0}^{b} \cos ^{2}\left(\frac{n \pi}{b} y\right) d y\right.\right. \\
& +\left(\frac{n \pi}{b}\right)^{2} \int_{0}^{a} \cos ^{2}\left(\frac{m \pi}{a} x\right) d x \int_{0}^{b} \sin ^{2}\left(\frac{n \pi}{b} y\right) d y \\
& \left.+2 \frac{m \pi}{a} \frac{n \pi}{b} \int_{0}^{a} \sin \left(\frac{m \pi}{a} x\right) \cos \left(\frac{m \pi}{a} x\right) d x \int_{0}^{b} \sin \left(\frac{n \pi}{b} y\right) \cos \left(\frac{n \pi}{b} y\right) d y\right\} \\
& -\frac{\beta_{z}}{k_{c, m n}^{2}} \int_{0}^{L} \sin \left(\frac{p \pi}{L} z\right) \cos \left(\frac{p \pi}{L} z\right) d z\left\{2 \frac{m \pi}{a} \int_{0}^{a} \sin \left(\frac{m \pi}{a} x\right) \cos \left(\frac{m \pi}{a} x\right) d x \int_{0}^{b} \cos ^{2}\left(\frac{n \pi}{b} y\right) d y\right. \\
& \left.+2 \frac{n \pi}{b} \int_{0}^{a} \cos ^{2}\left(\frac{m \pi}{a} x\right) d x \int_{0}^{b} \sin \left(\frac{n \pi}{b} y\right) \cos \left(\frac{n \pi}{b} y\right) d y\right\} \\
& \left.+\int_{0}^{a} \cos ^{2}\left(\frac{m \pi}{a} x\right) d x \int_{0}^{b} \cos ^{2}\left(\frac{n \pi}{b} y\right) d y \int_{0}^{L} \sin ^{2}\left(\frac{p \pi}{L} z\right) d z\right\}
\end{aligned}
$$

Considering Equations C.3, C.4 and C.7 the electric energy equation for the $\mathrm{TM}_{m n p}$ modes is finally:

$$
W_{m}=\frac{\mu_{0}}{4} A_{m n}^{2} \frac{a b L}{8} \varepsilon_{m} \varepsilon_{n}\left(1+\frac{\beta_{z}^{2}}{k_{c, m n}^{2}}\right)
$$

with:

$$
\begin{gathered}
\varepsilon_{m}= \begin{cases}2, & m=0 \\
1, & m \neq 0\end{cases} \\
\varepsilon_{n}= \begin{cases}2, & n=0 \\
1, & n \neq 0\end{cases}
\end{gathered}
$$


In the case of $\mathrm{TM}_{m n p}$ modes:

$$
\begin{aligned}
W_{m} & =\frac{\mu_{0}}{4} \int_{V_{c}} B_{m n}^{2} \frac{\left(\omega \varepsilon_{0}\right)^{2}}{k_{c, m n}^{4}}\left[\frac{n \pi}{b} \sin \left(\frac{m \pi}{a} x\right) \cos \left(\frac{n \pi}{b} y\right) \cos \left(\frac{p \pi}{L} z\right)\right. \\
+ & \left.\frac{m \pi}{a} \cos \left(\frac{m \pi}{a} x\right) \sin \left(\frac{n \pi}{b} y\right) \cos \left(\frac{p \pi}{L} z\right)\right]^{2} d V
\end{aligned}
$$

Considering Equation C.2

$$
\begin{aligned}
W_{m} & =\frac{\mu_{0}}{4} B_{m n}^{2} \frac{\left(\omega \varepsilon_{0}\right)^{2}}{k_{c, m n}^{4}} \int_{0}^{L} \cos ^{2}\left(\frac{p \pi}{L} z\right) d z\left\{\left(\frac{n \pi}{b}\right)^{2} \int_{0}^{a} \sin ^{2}\left(\frac{m \pi}{a} x\right) d x \int_{0}^{b} \cos ^{2}\left(\frac{n \pi}{b} y\right) d y\right. \\
& +\left(\frac{m \pi}{a}\right)^{2} \int_{0}^{a} \cos ^{2}\left(\frac{m \pi}{a} x\right) d x \int_{0}^{b} \sin ^{2}\left(\frac{n \pi}{b} y\right) d y \\
& \left.+2 \frac{n \pi}{b} \frac{m \pi}{a} \int_{0}^{a} \sin \left(\frac{m \pi}{a} x\right) \cos \left(\frac{m \pi}{a} x\right) d x \int_{0}^{b} \sin \left(\frac{n \pi}{b} y\right) \cos \left(\frac{n \pi}{b} y\right) d y\right\}
\end{aligned}
$$

Considering Equations C.3, C.4 and C.7 the electric energy equation for the $\mathrm{TM}_{m n p}$ modes is finally:

$$
W_{m}=\frac{\mu_{0}}{4} \frac{B_{m n}^{2} \omega_{u}^{2} \varepsilon_{0}^{2}}{k_{c, m n}^{2}} \frac{a b L}{8} \varepsilon_{p}
$$

with:

$$
\varepsilon_{p}= \begin{cases}2, & p=0 \\ 1, & p \neq 0\end{cases}
$$

\section{C.2 Calculation of Power in a Rectangular Empty Ca- vity}

The real dissipated power in the walls of the cavity is proportional to its surface and is calculated as

$$
P_{0}=\frac{R_{s}}{2} \int_{S_{c}}\left|\vec{H}_{\tan }\right|^{2} d S
$$

The field Equations 4.13 and 4.14 are applied to solve the $P_{0}$ expression.

$$
\begin{aligned}
P_{0} & =\frac{R_{s}}{2}\left\{2 \int_{0}^{a} \int_{0}^{b}\left(+\hat{a}_{y} H_{x}-\left.\hat{a}_{x} H_{y}\right|_{z=0}\right)^{2} d x d y\right. \\
& +2 \int_{0}^{a} \int_{0}^{L}\left(+\hat{a}_{x} H_{z}-\left.\hat{a}_{z} H_{x}\right|_{y=0}\right)^{2} d x d z \\
& \left.+2 \int_{0}^{b} \int_{0}^{L}\left(+\hat{a}_{z} H_{y}-\left.\hat{a}_{y} H_{z}\right|_{x=0}\right)^{2} d y d z\right\}
\end{aligned}
$$


Then, considering that $z=0$ implies $\cos \left(\frac{p \pi}{L} z\right)=1, y=0$ implies $\cos \left(\frac{n \pi}{b} y\right)=$ 1 and $x=0$ implies $\cos \left(\frac{m \pi}{a} x\right)=1$, for the case of $\mathrm{TE}_{m n p}$ modes:

$$
\begin{aligned}
P_{0} & =R_{s} A_{m n}^{2}\left\{\int_{0}^{a} \int_{0}^{b} \frac{\beta_{z}^{2}}{k_{m n}^{4}}\left[\frac{m \pi}{a} \sin \left(\frac{m \pi}{a} x\right) \cos \left(\frac{n \pi}{b} y\right)-\frac{n \pi}{b} \cos \left(\frac{m \pi}{a} x\right) \sin \left(\frac{n \pi}{b} y\right)\right]^{2} d x d y\right. \\
& +\int_{0}^{a} \int_{0}^{L}\left[\cos \left(\frac{m \pi}{a} x\right) \sin \left(\frac{p \pi}{L} z\right)+\frac{\beta_{z}}{k_{m n}^{2}} \frac{m \pi}{a} \sin \left(\frac{m \pi}{a} x\right) \cos \left(\frac{p \pi}{L} z\right)\right]^{2} d x d z \\
& \left.+\int_{0}^{b} \int_{0}^{L}-\left[\frac{\beta_{z}}{k_{m n}^{2}} \frac{n \pi}{b} \sin \left(\frac{n \pi}{b} y\right) \cos \left(\frac{p \pi}{L} z\right)-\cos \left(\frac{n \pi}{b} y\right) \sin \left(\frac{p \pi}{L} z\right)\right]^{2} d y d z\right\}
\end{aligned}
$$

Applying Equation C.2 and considering Equations C.3, C.4 and C.7 the equation is simplified as

$$
\begin{aligned}
P_{0} & =R_{s} A_{m n}^{2}\left\{\frac{\beta_{z}^{2}}{k_{m n}^{4}}\left[\left(\frac{m \pi}{a}\right)^{2} \frac{a b}{4} \varepsilon_{n}+\left(\frac{n \pi}{b}\right)^{2} \frac{a b}{4} \varepsilon_{m}\right]\right. \\
& \left.+\left[\frac{a L}{4} \varepsilon_{m}+\frac{\beta_{z}^{2}}{k_{m n}^{4}}\left(\frac{m \pi}{a}\right)^{2} \frac{a L}{4} \varepsilon_{p}\right]+\left[\frac{\beta_{z}^{2}}{k_{m n}^{4}}\left(\frac{n \pi}{b}\right)^{2} \frac{b L}{4} \varepsilon_{p}+\frac{b L}{4} \varepsilon_{n}\right]\right\}
\end{aligned}
$$

Considering that for $\mathrm{TE}_{m n p}$ modes $(m=0,1,2, \ldots, n=0,1,2, \ldots(m=$ $n \neq 0)$ and $p=1,2,3, \ldots)$ an analytical simplification is done and the dissipated power equation is finally:

$$
P_{0}=R_{s} A_{m n}^{2}\left\{\frac{\beta_{z}^{2}}{k_{c, m n}^{4}}\left[\frac{a b}{4} \varepsilon_{m} \varepsilon_{n} k_{c, m n}^{2}+\frac{L}{4}\left(\frac{(m \pi)^{2}}{a}+\frac{(n \pi)^{2}}{b}\right)\right]+\frac{L}{4}\left(a \varepsilon_{m}+b \varepsilon_{n}\right)\right\}
$$

with:

$$
\begin{gathered}
\varepsilon_{m}= \begin{cases}2, & m=0 \\
1, & m \neq 0\end{cases} \\
\varepsilon_{n}= \begin{cases}2, & n=0 \\
1, & n \neq 0\end{cases}
\end{gathered}
$$

In the case of $\mathrm{TM}_{m n p}$ modes, considering that $z=0$ implies $\cos \left(\frac{p \pi}{L} z\right)=1$, $y=0$ implies $\cos \left(\frac{n \pi}{b} y\right)=1$ and that $x=0$ implies $\cos \left(\frac{m \pi}{a} x\right)=1$ the dissipated power is developed as

$$
\begin{aligned}
P_{0} & =R_{s} B_{m n}^{2} \frac{\omega^{2} \varepsilon^{2}}{k_{c, m n}^{4}}\left\{\int_{0}^{a} \int_{0}^{b}\left[\frac{n \pi}{b} \sin \left(\frac{m \pi}{a} x\right) \cos \left(\frac{n \pi}{b} y\right)+\frac{m \pi}{a} \cos \left(\frac{m \pi}{a} x\right) \sin \left(\frac{n \pi}{b} y\right)\right]^{2} d x d y\right. \\
& +\int_{0}^{a} \int_{0}^{L}\left[\frac{n \pi}{b} \sin \left(\frac{m \pi}{a} x\right) \cos \left(\frac{p \pi}{L} z\right)\right]^{2} d x d z \\
& \left.+\int_{0}^{b} \int_{0}^{L}\left[\frac{m \pi}{a} \sin \left(\frac{n \pi}{b} y\right) \cos \left(\frac{p \pi}{L} z\right)\right]^{2} d y d z\right\}
\end{aligned}
$$


Equations C.2, C.3, C.4 and C.7 are considered to solve the final equation for the $\mathrm{TM}_{m n p}$ modes with $(m=1,2,3, \ldots, n=1,2,3, \ldots$ and $p=$ $0,1,2, \ldots)$ as

$$
P_{0}=R_{s} \frac{B_{m n}^{2} \omega^{2} \varepsilon_{0}^{2}}{k_{c, m n}^{4}}\left\{\frac{a b}{4} k_{c, m n}^{2}+\frac{L}{4} \varepsilon_{p}\left[b\left(\frac{m \pi}{a}\right)^{2}+a\left(\frac{n \pi}{b}\right)^{2}\right]\right\}
$$

with:

$$
\varepsilon_{p}= \begin{cases}2, & p=0 \\ 1, & p \neq 0\end{cases}
$$






\section{Bessel Function}

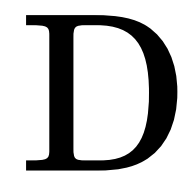

Bessel functions are important for finding separable solutions to the Laplace or Helmholtz equations in cylindrical and spherical coordinates. These functions are used in wave propagation equations as the electromagnetic waves travelling in a cylindrical waveguide or cavity.

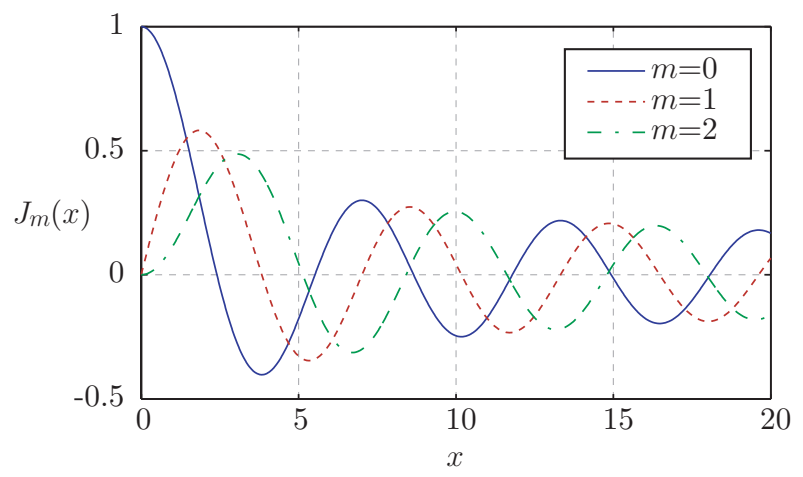

Figure D.1. Bessel function of 1st order $J_{m}(x)$ for $m=$ $0,1,2$. 
When the solutions of the Bessel equation are finite at the origin $(x=$ 0 ), they are designated Bessel functions of the first kind [97]. Figure D.1 represents this function for $m=0,1,2$. Every zero of the function, i.e. every time the function crosses the 0 value, gives orderly $(n=1,2,3 \ldots)$ the value of the function used for the calculation of the fields in a $\mathrm{TE}_{m n}$ or $\mathrm{TM}_{m n}$ mode.

Tables D.1 and D.2 collect the zeros of the Bessel function of the first kind $J_{m}(x)$ and its derivative $J_{m}^{\prime}(x)$ respectively, which are used in the calculation of the electromagnetic field equations in a cylindrical cavity. 


\begin{tabular}{l|rrrrr}
\hline$m \backslash n$ & $n=1$ & $n=2$ & $n=3$ & $n=4$ & $n=5$ \\
\hline$m=0$ & 2.4048 & 5.5201 & 8.6537 & 11.7915 & 14.9310 \\
$m=1$ & 3.8317 & 7.0156 & 10.1735 & 13.3237 & 16.4706 \\
$m=2$ & 5.1356 & 8.4172 & 11.6198 & 14.7960 & 17.9598 \\
$m=3$ & 6.3802 & 9.7610 & 13.0152 & 16.2235 & 19.4094 \\
$m=4$ & 7.5883 & 11.0647 & 14.3725 & 17.6160 & 20.8269 \\
$m=5$ & 8.7715 & 12.3386 & 15.7002 & 18.9801 & 22.2178 \\
$m=6$ & 9.9361 & 13.5893 & 17.0038 & 20.3208 & 23.5861 \\
$m=7$ & 11.0864 & 14.8213 & 18.2876 & 21.6415 & 24.9349 \\
$m=8$ & 12.2251 & 16.0378 & 19.5545 & 22.9452 & 26.2668 \\
$m=9$ & 13.3543 & 17.2412 & 20.8070 & 24.2339 & 27.5837 \\
$m=10$ & 14.4755 & 18.4335 & 22.0470 & 25.5094 & 28.8874 \\
$m=11$ & 15.5899 & 19.6160 & 23.2759 & 26.7733 & 30.1791 \\
$m=12$ & 16.6983 & 20.7899 & 24.4949 & 28.0267 & 31.4600 \\
$m=13$ & 17.8014 & 21.9562 & 25.7051 & 29.2706 & 32.7311 \\
$m=14$ & 18.9000 & 23.1158 & 26.9074 & 30.5060 & 33.9932 \\
$m=15$ & 19.9944 & 24.2692 & 28.1024 & 31.7334 & 35.2471 \\
$m=16$ & 21.0851 & 25.4170 & 29.2909 & 32.9536 & 36.4934 \\
$m=17$ & 22.1725 & 26.5598 & 30.4733 & 34.1673 & 37.7327 \\
$m=18$ & 23.2568 & 27.6979 & 31.6501 & 35.3747 & 38.9654 \\
$m=19$ & 24.3383 & 28.8317 & 32.8218 & 36.5765 & 40.1921 \\
$m=20$ & 25.4171 & 29.9616 & 33.9887 & 37.7729 & 41.4131 \\
\hline
\end{tabular}

Table D.1. Zeros $P_{m n}\left(J_{m}\left(P_{m n}\right)=0 n=1,2,3, \ldots\right)$ of the Bessel function of the first kind $J_{m}(x)$. 


\begin{tabular}{l|rrrrr}
\hline$m \backslash n$ & $n=1$ & $n=2$ & $n=3$ & $n=4$ & $n=5$ \\
\hline$m=0$ & 3.8317 & 7.0156 & 10.1735 & 13.3237 & 16.4706 \\
$m=1$ & 1.8412 & 5.3314 & 8.5363 & 11.7060 & 14.8636 \\
$m=2$ & 3.0542 & 6.7061 & 9.9695 & 13.1704 & 16.3475 \\
$m=3$ & 4.2012 & 8.0152 & 11.3459 & 14.5858 & 17.7887 \\
$m=4$ & 5.3176 & 9.2824 & 12.6819 & 15.9641 & 19.1960 \\
$m=5$ & 6.4156 & 10.5199 & 13.9872 & 17.3128 & 20.5755 \\
$m=6$ & 7.5013 & 11.7349 & 15.2682 & 18.6374 & 21.9317 \\
$m=7$ & 8.5778 & 12.9324 & 16.5294 & 19.9419 & 23.2681 \\
$m=8$ & 9.6474 & 14.1155 & 17.7740 & 21.2291 & 24.5872 \\
$m=9$ & 10.7114 & 15.2867 & 19.0046 & 22.5014 & 25.8913 \\
$m=10$ & 11.7709 & 16.4479 & 20.2230 & 23.7607 & 27.1820 \\
$m=11$ & 12.8265 & 17.6003 & 21.4309 & 25.0085 & 28.4609 \\
$m=12$ & 13.8788 & 18.7451 & 22.6293 & 26.2460 & 29.7290 \\
$m=13$ & 14.9284 & 19.8832 & 23.8194 & 27.4743 & 30.9874 \\
$m=14$ & 15.9754 & 21.0154 & 25.0020 & 28.6943 & 32.2370 \\
$m=15$ & 17.0203 & 22.1422 & 26.1778 & 29.9066 & 33.4784 \\
$m=16$ & 18.0633 & 23.2643 & 27.3474 & 31.1119 & 34.7125 \\
$m=17$ & 19.1045 & 24.3819 & 28.5114 & 32.3109 & 35.9396 \\
$m=18$ & 20.1441 & 25.4956 & 29.6701 & 33.5039 & 37.1604 \\
$m=19$ & 21.1823 & 26.6055 & 30.8241 & 34.6915 & 38.3752 \\
$m=20$ & 22.2191 & 27.7121 & 31.9737 & 35.8739 & 39.5845 \\
\hline
\end{tabular}

Table D.2. Zeros $P_{m n}^{\prime}$ of derivative $J_{m}^{\prime}\left(P_{m n}^{\prime}\right)=0(n=$ $1,2,3, \ldots)$ of the Bessel function of the first kind $J_{m}(x)$. 


\section{Bibliography}

[1] A. Strasser and P. Rudling, Fuel Fabrication Process Handbook. ANT International, 2005.

[2] M. Salvatores and G. Palmiotti, "Radioactive waste partitioning and transmutation within advanced fuel cycles: Achievements and challenges," Progress in Particle and Nuclear Physics, vol. 66, pp. 144-166, 2011.

[3] NEA, "Potential benefits and impacts of advanced nuclear fuel cycles with actinide partitioning and transmutation," OECD/NEA, Tech. Rep., 2011.

[4] K. Tsujimoto, T. Sasa, K. Nishihara, T. Takizuka, and H. Takano, "Accelerator-drivensystem for transmutation of high-level waste," Progress in Nuclear Energy, vol. 37, pp. 339-344, 2000.

[5] M. Pouchon, M. Nakamura, and C. Hellwig, "A mixed ceramic-metal sphere-pac concept," Journal of Nuclear Science and Technology Supplement, vol. 3, pp. 830-833, 2002.

[6] M. Pouchon, M. Nakamura, C. Hellwig, F. Ingold, and C. Degueldre, 
"Cermet sphere-pac concept for inert matrix fuel," Journal of Nuclear Materials, vol. 319, pp. 37-43, 2003.

[7] M. Pouchon and F. Ingold, "Internal gelation at PSI: The past and the future," in Proceedings of the 2009 International Congress on Advances in Nuclear Power Plants, 2009.

[8] F. van der Brugghen, A. J. Noothout, M. Hermans, J. Kanji, and O. Votocek, "A U(VI)-process for microspheres production," Symposium on Sol-Gel Processes and Reactor Fuel Cycles, Gatlinburg, TN, CONF-700502, pp. 253-263, 1970.

[9] J. Kanij, A. Noothout, and O. Votocek, "The KEMA U(IV) process for production of $\mathrm{UO}_{2}$ microsphere," Sol-Gel Process for Fuel Fabrication IAEA, vol. 161, pp. 185-195, 1974.

[10] G. Ledergerber, "Internal gelation for oxide and nitride particles," JAERI, Tech. Rep., 1996.

[11] H. Daniels, S. Neumeier, A. Bukaemskiy, G. Modolo, and D. Bosbach, "Fabrication of oxidic uranium-neodymium microspheres by internal gelation," Progress in Nuclear Energy, vol. 57, pp. 106-110, 2012.

[12] K. Knotik, P. Leichter, E. Bonek, G. Magerl, and H. Huschka, "Herstellung von HTR-Brennstoffkernen durch Mikrowellenhärtung von wässrigen Metallsalz-Kunstharztrpfchen," PSI, Tech. Rep., 1981.

[13] G. Ledergerber, "Improvements of the internal gelation process." Transactions of the American Nuclear Society, 40, 55-56, Tech. Rep., 1982.

[14] — , "Internal gelation using microwaves," IAEA-TECDOC, Advanced Fuel Technology and Performance (Proceedings of an Advisory Group Meeting, Würenlingen, Switzerland, 4-6 Dec. 1984, 352, 165-174), Tech. Rep., 1985.

[15] S. Yamagishi, A. Hasegawa, and T. Ogawa, "Development of a rapid gelation apparatus with microwave heating," Japan Atomic Energy Research Institute, Tech. Rep., 1994. 
[16] S. Yamagishi and A. Hasegawa, "Method and apparatus for producing gel particles," U.S. Patent 5,581,589, 1996.

[17] S. Yamagishi, "A new internal gelation process for fuel microsphere preparation without cooling initial solutions," Journal of Nuclear Materials, vol. 254, pp. 14-21, 1998.

[18] A. Rosin, A. Schmidt, T. Gerdes, J. Somers, and M. Willert-Porada, "Microwave assisted internal gelation of droplets - a case study," InVerTec e.V. and Institute for Transuranium Elements and University of Bayreuth, Tech. Rep., 2005.

[19] J. Morrisson and M. Cross, "Scattering of a plane electromagnetic wave by axisymmetric raindrops," The Bell System Technical Journal 6, July-August 1974.

[20] E. Bonek, K. Knotik, P. Leichter, G. Magerl, and L. Rohrecker, "Microwave hardening of free-falling radioactive droplets," 12th European Microwave conference, 1982.

[21] L. A. Campañone and N. E. Zaritzky, "Mathematical analysis of microwave heating process," J. Food Eng., vol. 69, pp. 359-368, 2005.

[22] F. Gardiol, "Heating with microwaves - an introduction," Chaire d'Electromagnétisme et d'Hyperfréquences (EHF) EPFL Lausanne, Switzerland, Tech. Rep., 1977.

[23] D. Issadore, K. H. Humphry, K. A. Brown, L. Sandberg, D. A. Weitz, and R. M. Westervelt, "Microwave dielectric heating of drops in microfluidic devices," Lab Chip, vol. 9, pp. 1701-1706, 2009.

[24] D. C. Kincaid and L. T. S., "A water droplet evaporation and temperature model," Transactions of the ASAE, vol. 32, pp. 457-463, 1989.

[25] NEA, "Advanced nuclear fuel cycles and radioactive waste management," OECD/NEA, Tech. Rep., 2006.

[26] IAEA, Geochemical exploration for uranium: Technical Reports Series 284, I. Vienna, Ed. International Atomic Energy Agency, 1988. 
[27] — Analytical techniques in uranium exploration and ore processing: Technical Reports Series 341, I. Vienna, Ed. International Atomic Energy Agency, 1992.

[28] — Minimization of waste from uranium purification, enrichment and fuel fabrication, I. Vienna, Ed. International Atomic Energy Agency, 1999.

[29] - Status of advanced light water reactor designs. IAEA-TECDOC1391, I. Vienna, Ed. International Atomic Energy Agency, 2004.

[30] — - World Distribution of Uranium Deposits (UDEPO) with Uranium Deposit Classification, I. Vienna, Ed. International Atomic Energy Agency, 2009.

[31] _ Establishment of Uranium Mining and Processing Operations in the Context of Sustainable Development, I. Vienna, Ed. International Atomic Energy Agency, 2009.

[32] V. Arkhipov, "Future nuclear energy systems: Generating electricity, burning wastes," IAEA Bulletin 39/2/1997.

[33] NAGRA (National Cooperative for the Disposal of Radioactive Waste), "Project opalinius clay: Safety report: Demonstration of disposal feasibility for spent fuel, vitrified high-level waste and long-lived intermediate-level waste," NAGRA, Wettingen, Technical Report 0205, 2002 .

[34] ICRP, "Age-dependent doses to members of the public from intake of radionuclides, part 5, compilation of ingestion and inhalation coefficients, Publication 72," Annals of the ICRP, vol. 26/1, 1996.

[35] M. Kushner, "Nuclear fuel fabrication for commercial electric power generation," IEEE Transactions on Power Apparatus and Systems, vol. PAS-93, Issue: 1, pp. $244-247,1974$.

[36] Y. Suzuki, T. Sasayama, Y. Arai, and H. Watanabe, "Fabrication of uranium-plutonium mixed carbide pellets," Journal of Nuclear Science and Technology, vol. 18:1, pp. 61-70, 1981. 
[37] J. Hauth, "Vibration-compacted ceramic fuel elements, HW-60346," General Electric Company; Handford Atomic Products Operation: Richland, WA, Tech. Rep., 1959.

[38] J. Hauth and R. Anicetti, "Vibrational compaction of ceramic powders for nuclear fuel elements, hw-sa-1735," United States Atomic Energy Commission: Washington, DC, Tech. Rep., 1959.

[39] M. Pouchon, G. Ledergerber, F. Ingold, and K. Bakker, Sphere-Pac and VIPAC Fuel. Elsevier, 2012, ch. 3.11, pp. 275-312.

[40] R. Wymer, "Laboratory and engineering studies of sol-gel processes at ORNL," In Proceedings of a Panel on "Sol-Gel Processes for Ceramic Nuclear Fuels" IAEA: Viena, pp. 131-172, 1968.

[41] H. Nickel, H. Nabielek, G. Pott, and A. Mehner, "Long time experience with the development of HTR fuel elements in Germany," Nuclear Engineering and Design, vol. 217, p. 141151, 2002.

[42] C. Jungo and G. Ledergerber, "Process and apparatus for producing microspheres," Patent 4431 164, 1984.

[43] C. Cozzo, S. Vaucher, K. Ishizaki, D. Megias-Alguacil, and M. A. Pouchon, "Chemistry of uranium surrogate during microwave assisted internal gelation for fuel fabrication," Proc. of GLOBAL 2011, 2011.

[44] Y. W. Lee, H. S. Kim, C. Joung, S. Na, G. Ledergerber, P. Heimgartner, M. Pouchon, and M. Burghartz, "Preparation of simulated inert matrix fuel with different powders by dry milling method," Journal of Nuclear Materials, vol. 274, pp. 7-14, 1999.

[45] M. Hermans, J. Kanij, A. Noothout, and T. van der Plas, "Status report from the Netherlands: Sol-gel fuel studies," in Sol-Gel Processes for Ceramic Nuclear Fuels, I. Vienna, Ed., International Atomic Energy Agency. IAEA Vienna, December 1968, p. 190.

[46] G. Ledergerber, H. Alder, F. Ingold, and R. Stratton, "Experience in preparing nuclear fuel by the gelation method," 1986. 
[47] M. Pouchon, F. Ingold, Z. Kopajtic, Y. Tomita, and S. Kono, "Fabrication and characterization of MOX microspheres for the FUJI project," in Proceedings of the Global 2003, New Orleans, LA, 2003, pp. 653657.

[48] W. Rohsenow, J. Hartnett, and Y. Cho, Handbook of Heat Transfer, 3rd ed., Y. C. W.M. Rohsenow, J.P. Hartnett, Ed. McGraw-Hill, 1998.

[49] A. Metaxas and R. Meredith, Industrial Microwave Heating, ser. IEE Power Engineering Series 4. Peter Peregrinus Ltd., London, UK, 1983.

[50] G. Roussy and J. Pearce, Foundations and Industrial Applications of Microwaves and Radio Frequency Fields. John Wiley \& Sons, Inc., 1995.

[51] J. Crank and P. Nicolson, "A practical method for numerical evaluation of solutions of partial differential equations of the heat conduction type," Proc. Camb. Phil. Soc., vol. 43, pp. 50-67, 1947.

[52] S. J. Farlow, Partial Differential Equations for Scientists and Engineers. John Wiley \& Sons, Inc. \& Dover Publications, Inc., 1982, 1993.

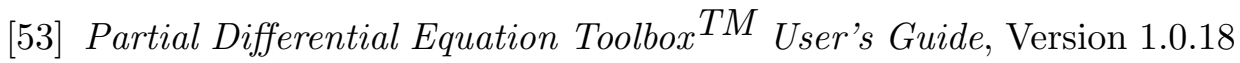
(release 2011a) ed., The MathWorks, Inc., April 2011.

[54] F. P. Incropera, D. P. DeWitt, T. L. Bergman, and A. S. Lavine, Introduction to heat transfer, 5th ed. Wiley, 2007.

[55] M. Cabanes-Sempere, J. M. Catalá-Civera, C. Cozzo, S. Vaucher, and M. A. Pouchon, "Innovative production of nuclear fuel by microwave internal gelation: Heat transfer model of falling droplets," Progress in Nuclear Energy, vol. 57, pp. 111-116, 2012.

[56] C. Balanis, Advanced Engineering Electromagnetics. John Wiley \& Sons, Inc., 1989.

[57] D. M. Pozar, Microwave Engineering, third edition ed., Wiley, Ed. John Wiley \& Sons, Inc., 2005. 
[58] A. J. Canós, J. M. Catalá-Civera, F. L. Peñaranda-Foix, and E. de los Reyes-Davó, "A novel technique for deembeding the unloaded resonance frequency from measurements of microwave cavities," IEEE Transactions on Microwave Theory and Techniques, vol. 54, no. 8, pp. 3407-3416, August 2006.

[59] D. Kajfez, Q-Factor. Oxford MS: Vector Fields, 1994.

[60] A. J. Canós, J. M. Catalá-Civera, F. Peñaranda-Foix, J. MonzóCabrera, and E. de los Reyes-Davó, "A new empirical method for extracting unloaded resonant frequencies from microwave resonant cavities," IEEE MTT-S Int. Microw. Symp. Dig., pp. 1823-1825, 2003.

[61] QuickWave Editor and Simulator Reference Guide, v.6.5 ed., QWEDQW3D, Warsaw, 2006.

[62] CST Microwave Studio, CST - Computer Simulation Technology AG, 2012, www.cst.com.

[63] H. Bethe and J. Schwinger, "Perturbation theory for cavities," National Defense Research Committee, Tech. Rep. D1-117, 4th March 1943.

[64] R. A. Waldrom, Theory of Guided Electromagnetic Waves (Chapter IV). London: Van Nostrand Reinhold Company, 2004.

[65] L. F. Chen, C. K. Ong, C. P. Neo, V. V. Varadan, and V. K. Varadan, Microwave Electronics: Measurement and Materials Characterization, Chapter 6. New York: J. Wiley \& Sons, 2004.

[66] E. G. Spencer, R. LeCraw, and L. Ault, "Note on cavity perturbation theory," J. Appl. Phys, vol. 28, no. 1, pp. 130-132, January 1957.

[67] A. Bonincontro and C. Cametti, "On the applicability of the cavity perturbation method to high-loss dielectrics," J. Phys. E: Sci. Instrum., vol. 10, pp. 1232-1233, 1977.

[68] W. Bauhofer, "Determination of semiconductor energy gaps using the microwave cavity perturbation method," J. Phys. E: Sci. Instrum, vol. 14, pp. 934-938, March 1981. 
[69] R. F. Harrington, Field Computation by Moment methods. USA: Oxford University Press, 1993.

[70] U. Kaatze, "Complex permittivity of water as a function of frequency and temperature," J. Chem. Eng. Data, vol. 34, no. 4, pp. 371-374, 1989 .

[71] M. Cabanes-Sempere, J. M. Catalá-Civera, C. Cozzo, S. Vaucher, and M. A. Pouchon, "Innovative production of nuclear fuel by microwave internal gelation: Theoretical microwaves-microspheres interaction," in Proceedings of the 13th International Conference on Microwave and RF Heating. Toulouse, France: Ampere, September 5-9th 2011, pp. 231-234, ISBN: 978.2.85428.978.7.

[72] M. Cabanes-Sempere, A. J. Canós, J. M. Catalá-Civera, F. L. Peñaranda-Foix, C. Cozzo, and M. A. Pouchon, "Power and thermal study of a single drop heated while passing through a $\mathrm{TE}_{10 p}$ rectangular cavity," in Proceedings of the International Microwave Symposium IMS 2013, Seattle (USA), June 2-7th 2013.

[73] Basics of measuring the dielectric properties of materials, literature number 5091-3300e ed., Hewlett Packard, 1992, http://www.scribd.com/doc/10152564/HPAN12171-Basics-ofMeasuring-the-Dielectric-Properties-of-Materials.

[74] H. Fröhlich, Theory of Dielectrics, Dielectric Constant and Dielectric Loss (Monographs on the physics and chemistry of materials), 2nd ed., O. U. Press, Ed., 1987, iSBN 0-19-851379-8.

[75] J. Baker-Jarvis, M. D. Janezic, and D. C. DeGroot, "High-frequency dielectric measurements," IEEE Instrumentation and Measurement Magazine, vol. 13, no. 2, pp. 24-31, April 2010.

[76] M. D. Janezic and J. Baker-Jarvis, "Full-wave analysis of a splitcylinder resonator for nondestructive permittivity measurements," IEEE Trans. Microwave Theory \& Tech., vol. 47, no. 10, pp. 20142020, October 1999. 
[77] J. M. Catalá-Civera, J. D. Gutiérrez-Cano, F. L. Peñaranda-Foix, and B. García-Baños, "Portable system for dielectric characterization of materials at microwave frequencies," in Proceedings of the 13th International Conference on Microwave and RF Heating. Toulouse, France: Ampere, September 5-9th 2011, pp. 127-130, ISBN: 978.2.85428.978.7.

[78] F. L. Peñaranda-Foix and J. M. Catalá-Civera, "Circuital analysis of cylindrical structures applied to the electromagnetic resolution of resonant cavities," Chapter 7 in Passive Microwave Components and Antennas, pp. 141-168, April 2010, ISBN 978-953-307-083-4 Hardcopy and Online paper are available at webpage: http://sciyo.com/books/show/title/passive-microwavecomponents-and-antennas.

[79] F. L. Peñaranda-Foix, M. D. Janezic, J. M. Catalá-Civera, and A. J. Canós, "Full-wave analysis of dielectric-loaded cylindrical waveguides and cavities using a new four-port ring network," IEEE Trans. Microwave Theory \& Tech., vol. 60, no. 9, pp. 2730-2740, September 2012.

[80] J. Bobowski and T. Johnson, "Permittivity measurements of biological samples by an open-ended coaxial line," Progress In Electromagnetics Research B, vol. 40, pp. 159-183, 2012.

[81] U. Kaatze, "Reference liquids for the calibration of dielectric sensors and measurement instruments," Meas. Sci. Technol., vol. 18, pp. 967976, 2007.

[82] F. Bolla, "Chemical and dielectric studies of internal gelation reagents," Master's thesis, École Polytechnique Fédérale de Lausanne, January 2013.

[83] J. Baker-Jarvis, M. D. Janezic, P. D. Domich, and R. G. Geyer, "Analysis of an open-ended coaxial probe with lift-off for nondestructive testing," IEEE Trans. Intrum. Meas., vol. 43, no. 5, pp. 711-718, Oct. 1994. 
[84] S. Bell, Guide to Uncertainty of Measurement (Issue 2). National Physical Laboratory (NPL), Aug. 1999, issue 2 with amendments March 2001.

[85] E. B. Millard, Physical chemistry for colleges, 5th ed. McGraw-Hill Book Company, 1941.

[86] J. Wigren and P. Andersson, "Thermal conductivity and heat capacity of adamantane and hexamethylenetetramine under pressure," Mol. Cryst. Liq. Cryst., vol. 59, pp. 137-148, 1980.

[87] E. White, "Enthalpy composition diagram and other data for hexamine-water system," Journal of Chemical and Engineering Data, vol. 12, no. 3, pp. 285-289, July 1967, dOI:10.1021/je60034a001.

[88] M. Cabanes-Sempere, C. Cozzo, J. M. Catalá-Civera, F. L. PeñarandaFoix, K. Ishizaki, S. Vaucher, and M. A. Pouchon, "Characterization of free falling drops inside a microwave cavity," in Proceedings of the International Microwave Symposium IMS 2012, Montreal (Canada), June 17-22nd 2012, pp. 1738-1740, ISBN: 978.1.4673.10868.4. (DOI: 10.1109/MWSYM.2012.6259757).

[89] AR rf/microwave instrumentation: Model 250TR7z5G18, www.arworldwide.com, 160 School House Road, Souderton, PA 18964.

[90] K. Gotoh and J. L. Finney, "Statistical geometrical approach to random packing density of equal spheres," Nature, vol. 252, p. 202205, 1974 .

[91] C. Cozzo, M. Cabanes-Sempere, and M. A. Pouchon, "Methods of advanced waste conditioning by microwave internal gelation: Set up development and modeling," OCDE, 2013.

[92] W. E. Ranz and W. R. J. Marshall, "Evaporation from drops," Chemical Engineering Progress, vol. 48, pp. 141-180, 1952.

[93] R. Miller, K. Harstad, and J. Bellan, "Evaluation of equilibrium and non-equilibrium evaporation models for many-droplet gas-liquid flow 
simulations," Jet Propulsion Laboratory, California Institute of Technology, Tech. Rep., 1998.

[94] S. X. Q. Lin and X. D. Chen, "Improving the glass-filament method for accurate measurement of drying kinetics of liquid droplets," Trans IChemE, vol. 80, pp. 401-410, 2002.

[95] D.-I. J. Wilms, "Evaporation of multicomponent droplets," Ph.D. dissertation, Fakultät für Luft- und Raumfahrttechnik und Geodäsie der Universität Stuttgart, 2005.

[96] J. C. Dixon, The Shock Absorber Handbook, L. . P. E. P. L. John Wiley \& Sons, Ed. John Wiley \& Sons, Ltd, 2007.

[97] M. Abramowitz and I. A. Stegun, Handbook of Mathematical Functions with Formulas, Graphs, and Mathematical Tables. New York: Dover, 1964, ISBN 978-0486612720. 